\title{
Synthesis of Hemilabile Cyclic (Alkyl)(amino)carbenes (CAACs) and Applications in Organometallic Chemistry
}

Jiaxiang Chu, Dominik Munz, Rodolphe Jazzar, Mohand Melaimi, and Guy Bertrand*

UCSD-CNRS Joint Research Chemistry, La Jolla, CA 92093-0343, USA

E-mail address and telephone number:

guybertrand@ucsd.edu, +1 (858) 5345412

\section{Content:}

1. Experimental Details - General S2

2. Cyclic Iminium Salts S2

3. Free Carbenes $\quad$ S19

4. Copper and Gold Complexes $\quad$ S28

5. Catalysis $\quad$ S43

6. Solid State Structures $\quad$ S44

$\begin{array}{ll}\text { 7. References } & \text { S74 }\end{array}$ 


\section{Experimental Details - General}

General: ${ }^{1} \mathrm{H},{ }^{13} \mathrm{C}$ NMR, ${ }^{19} \mathrm{~F},{ }^{31} \mathrm{P}$ NMR spectra were collected on Bruker Avance 300, Jeol ECA 500 and Varian Inova 500 spectrometers. Chemical shifts are given in ppm and are referenced to $\mathrm{SiMe}_{4}\left({ }^{1} \mathrm{H},{ }^{13} \mathrm{C}\right)$ and $\mathrm{CFCl}_{3}\left({ }^{19} \mathrm{~F}\right)$ and $\mathrm{H}_{3} \mathrm{PO}_{4}\left({ }^{31} \mathrm{P}\right)$. Coupling constants $J$ are given in Hertz as positive values regardless of their real individual signs. NMR multiplicities are abbreviated as follows: $\mathrm{s}=$ singlet, $\mathrm{d}=$ doublet, $\mathrm{t}=$ triplet, $\mathrm{q}=$ quartet, $\mathrm{sept}=$ septet, $\mathrm{m}=$ multiplet, $\mathrm{br}=$ broad signal. All spectra were obtained at $25^{\circ} \mathrm{C}$ in the solvent indicated. Mass spectra were performed at the UC San Diego Mass Spectrometry Laboratory on an Agilent 6230 Accurate-Mass TOFMS spectrometer. Melting points were determined with an electrothermal MEL-TEMP apparatus. All compounds were - if not otherwise indicated prepared in a drybox or with Schlenk flask technique under an atmosphere of argon. THF, $\mathrm{Et}_{2} \mathrm{O}$, benzene, toluene were used freshly distilled from $\mathrm{Na} /$ benzophenone. Hexanes, pentanes, dichloromethane, chloroform and $\mathrm{MeCN}$ were used freshly distilled from calcium hydride. Compounds (CAAC)AuCl, ${ }^{1}$ (CAAC)CuCl, ${ }^{2}$ (CAAC)CuOTf, ${ }^{3}$ (CAAC)CuO ${ }^{t} \mathrm{Bu},{ }^{4}$ $\mathrm{Me}_{2} \mathrm{NCH}_{2} \mathrm{Cl},{ }^{5}{ }^{t} \mathrm{BuC}(\mathrm{Cl})(=\mathrm{NDipp})^{6}$ and biphenylene ${ }^{7}$ were prepared as reported in the literature, while all other starting materials were purchased from commercial sources and used without further purification.

\section{Cyclic Iminium Salts}

\section{$\underline{\text { Imine } 1}$}

A mixture of $\mathrm{MgSO}_{4}(24.07 \mathrm{~g}, 200 \mathrm{mmol}), \mathrm{DippNH}_{2}(35.46 \mathrm{~g}, 200 \mathrm{mmol})$, propionaldehyde $(17.42 \mathrm{~g}, 300 \mathrm{mmol})$, and $\mathrm{CH}_{2} \mathrm{Cl}_{2}(100 \mathrm{~mL})$ were stirred at room temperature overnight and then filtered. Removal of the volatiles

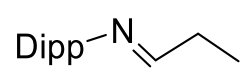
1 under vacuum afforded a pale yellow oil. The yield was quantitative and the product was directly used in the next step.

${ }^{1} \mathrm{H}$ NMR $\left(\mathrm{CDCl}_{3}, 400 \mathrm{MHz}, \mathrm{ppm}\right): \delta=7.64(\mathrm{t}, J=4.4 \mathrm{~Hz}, 1 \mathrm{H}, \operatorname{DippN}=\mathrm{CH}), 7.12-7.04(\mathrm{~m}$, $3 \mathrm{H}, \mathrm{ArH}), 2.92\left(\mathrm{~d}, J=6.8 \mathrm{~Hz}, 2 \mathrm{H}, \mathrm{C} H \mathrm{Me}_{2}\right), 2.53\left(\mathrm{~m}, 2 \mathrm{H}, \mathrm{CH}_{2}\right), 1.26\left(\mathrm{t}, J=6.8 \mathrm{~Hz}, 3 \mathrm{H}, \mathrm{CH}_{3}\right)$, $1.16(\mathrm{~d}, J=6.8 \mathrm{~Hz}, 12 \mathrm{H}, \mathrm{CHMe}) .{ }^{13} \mathrm{C}\left\{{ }^{1} \mathrm{H}\right\} \mathrm{NMR}\left(\mathrm{CDCl}_{3}, 125 \mathrm{MHz}, \mathrm{ppm}\right): \delta=168.3$ (imine C), 149.0, 137.7, 124.0, 123.0 (ArC), 30.0, 27.7, 23.6, 10.1. 


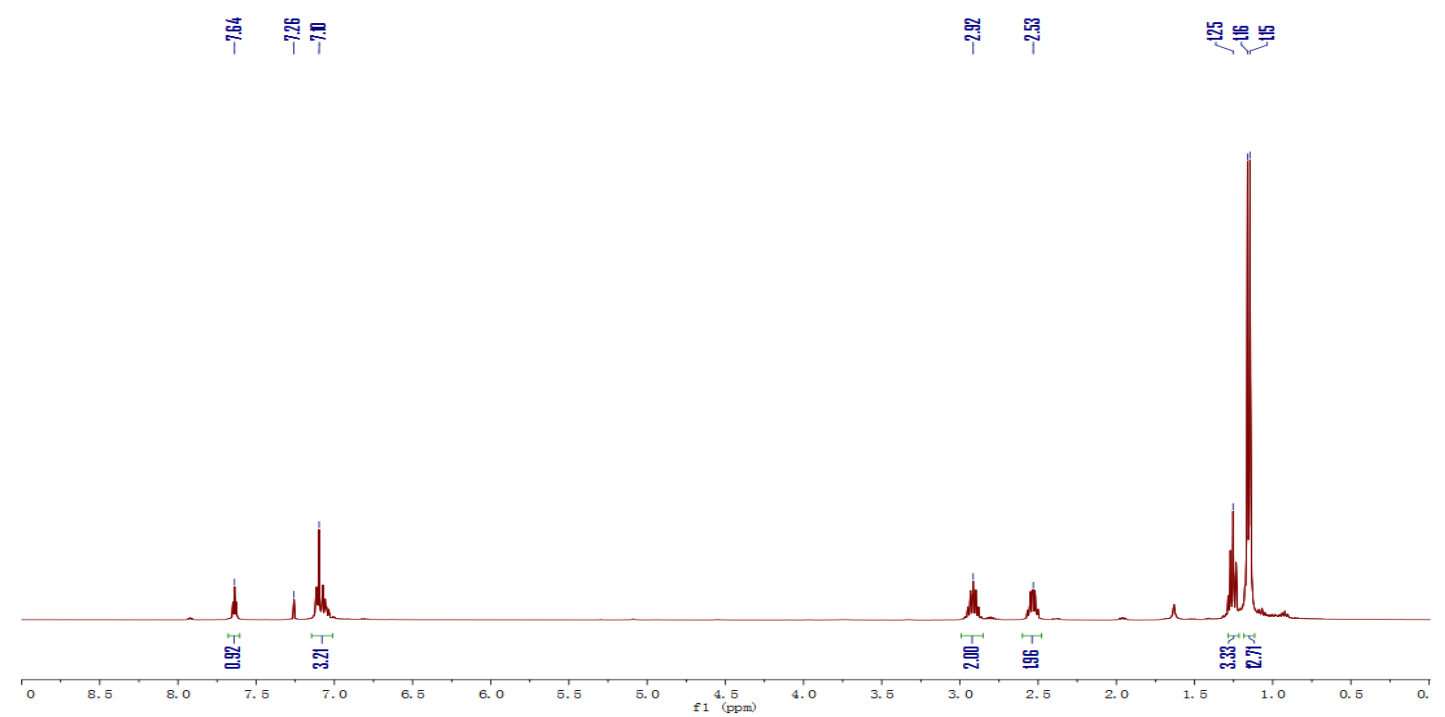

Figure S1. ${ }^{1} \mathrm{H}$ NMR of $1\left(\mathrm{CDCl}_{3}, 400 \mathrm{MHz}\right)$.
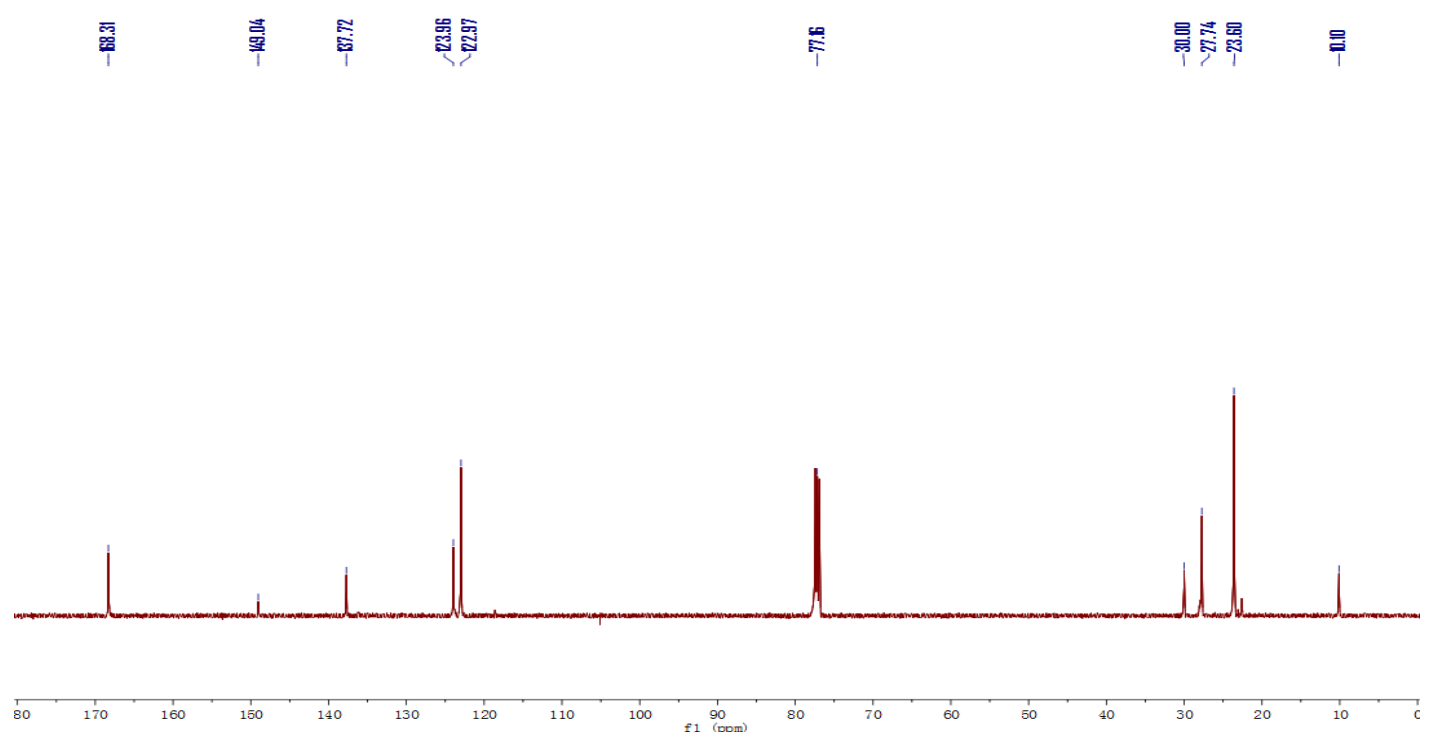

Figure S2. ${ }^{13} \mathrm{C}\left\{{ }^{1} \mathrm{H}\right\} \mathrm{NMR}$ of $\mathbf{1}\left(\mathrm{CDCl}_{3}, 125 \mathrm{MHz}\right)$.

\section{Imine 2}

A solution of ${ }^{n} \mathrm{BuLi}$ in hexanes $(98 \mathrm{~mL}, 2.5 \mathrm{M}, 245 \mathrm{mmol})$ was added at $-78{ }^{\circ} \mathrm{C}$ to a stirred solution of DippN $=\mathrm{CHCH}_{2} \mathrm{CH}_{3} 1(200 \mathrm{mmol}$, from the last step) in THF $(100 \mathrm{~mL})$. The solution was warmed to room temperature and stirred for $1 \mathrm{~h}$ and then cooled to $-78{ }^{\circ} \mathrm{C}$.<smiles>C=C(C)CC(C)/C=N/POc1ccccc1</smiles>
3-Chloro-2-methylpropene $(30 \mathrm{~mL}, 305 \mathrm{mmol})$ was added in three portions. The solution was slowly warmed to room temperature overnight. Removal of the volatiles under vacuum afforded a mixture of an oil (alkenyl aldimine 2) and a solid ( $\mathrm{LiCl}$ ). The yield was quantitative and the mixture was directly used in the next step.

${ }^{1} \mathrm{H}$ NMR $\left(\mathrm{CDCl}_{3}, 400 \mathrm{MHz}, \mathrm{ppm}\right): \delta=7.47(\mathrm{~d}, J=5.6 \mathrm{~Hz}, 1 \mathrm{H}, \mathrm{DippN}=\mathrm{CH}), 7.12-7.02(\mathrm{~m}$, $3 \mathrm{H}, \operatorname{Ar} H), 4.82\left(\mathrm{~d}, 1 \mathrm{H},=\mathrm{CH}_{2}\right), 4.76\left(\mathrm{~s}, 1 \mathrm{H},=\mathrm{CH}_{2}\right), 2.92\left(\mathrm{sept}, J=6.8 \mathrm{~Hz}, 2 \mathrm{H}, \mathrm{CHMe}_{2}\right), 2.88$ (m, 1H, CHMe), $2.41\left(\mathrm{dd}, J=14.4 \mathrm{~Hz}, J=8.0 \mathrm{~Hz}, 1 \mathrm{H}, \mathrm{CH}_{2}\right), 2.18(\mathrm{dd}, J=14.4 \mathrm{~Hz}, J=7.2$ 
$\left.\mathrm{Hz}, 1 \mathrm{H}, \mathrm{CH}_{2}\right), 1.80\left(\mathrm{~s}, 3 \mathrm{H}, \mathrm{CH}_{3}\right), 1.21(\mathrm{~d}, J=6.8 \mathrm{~Hz}, 3 \mathrm{H}, \mathrm{CHMe}), 1.15(\mathrm{~d}, J=6.8 \mathrm{~Hz}, 6 \mathrm{H}$, $\left.\mathrm{CHMe}_{2}\right), 1.13$ (d, $\left.\left.J=6.8 \mathrm{~Hz}, 6 \mathrm{H}, \mathrm{CHMe}\right)_{2}\right){ }^{13} \mathrm{C}\left\{{ }^{1} \mathrm{H}\right\} \mathrm{NMR}\left(\mathrm{CDCl}_{3}, 125 \mathrm{MHz}, \mathrm{ppm}\right): \delta=$ $171.2(\mathrm{CH}=\mathrm{NAr}), 148.8,143.2,137.7,124.0,122.9,112.6\left(\mathrm{ArC}\right.$ and $\left.C=\mathrm{CH}_{2}\right), 42.4,38.2,27.6$, 23.7, 23.5, 22.3, 17.3.

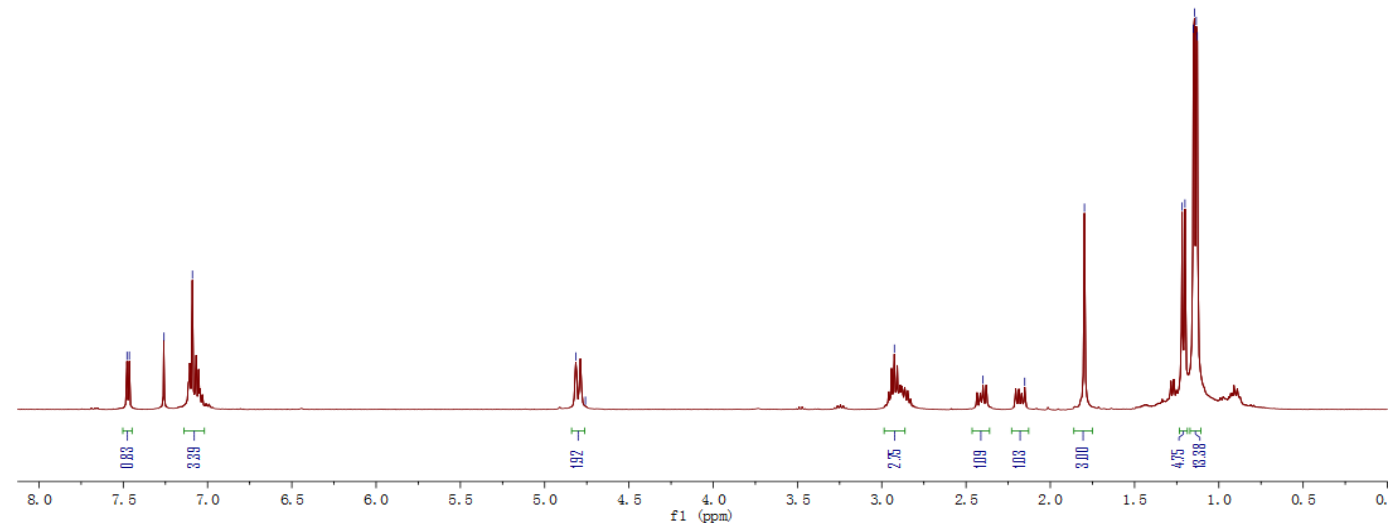

Figure S3. ${ }^{1} \mathrm{H} \mathrm{NMR}$ of $2\left(\mathrm{CDCl}_{3}, 400 \mathrm{MHz}\right)$.

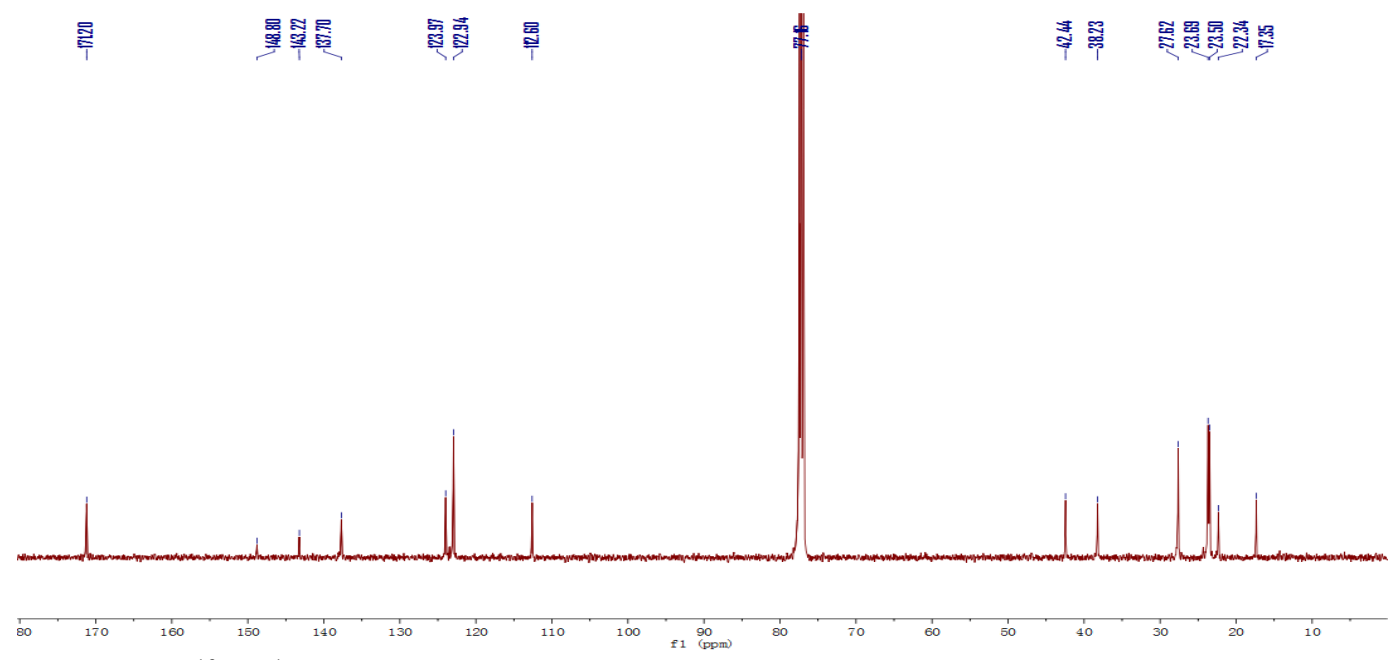

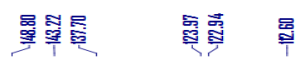

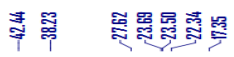

Figure S4. ${ }^{13} \mathrm{C}\left\{{ }^{1} \mathrm{H}\right\}$ NMR of $2\left(\mathrm{CDCl}_{3}, 125 \mathrm{MHz}\right)$.

\section{Imine 3a}

A solution of ${ }^{n} \mathrm{BuLi}(2.5 \mathrm{M})$ in hexanes $(69 \mathrm{~mL}, 172 \mathrm{mmol})$ was added at $-78{ }^{\circ} \mathrm{C}$ to a stirred solution of olefin $2(31.2 \mathrm{~g}, 115 \mathrm{mmol})$ in $\mathrm{Et}_{2} \mathrm{O}$ $(100 \mathrm{~mL})$. The solution was warmed to room temperature and stirred for $1 \mathrm{~h}$ and then cooled to $-78^{\circ} \mathrm{C}$. Chloromethyl ethylether $(17.1 \mathrm{~mL}, 17.4 \mathrm{~g}$, $184 \mathrm{mmol}$ ) was added. The solution was slowly warmed to room<smiles>C=C(C)CC(C)(C=Nc1ccccc1)COCC</smiles>
temperature overnight. Evaporation gave a mixture of $\mathrm{LiCl}$ and a yellow oil of approximately 
$90 \%$ purity, which was directly used for the next step without further purification.

${ }^{1} \mathrm{H}$ NMR $\left(\mathrm{CDCl}_{3}, 300 \mathrm{MHz}, \mathrm{ppm}\right): \delta=7.83$ (s, imine-H), 7.45-7.2 (m, 3H, $\left.\mathrm{ArH}\right), 5.1(\mathrm{~s}, 1 \mathrm{H}$, $=\mathrm{C} H), 4.96(\mathrm{~s}, 1 \mathrm{H},=\mathrm{CH}), 3.14\left(\mathrm{~s}, 2 \mathrm{H}, \mathrm{C} H \mathrm{Me}_{2}\right), 2.69\left(\mathrm{~d}, J=13.2 \mathrm{~Hz}, 1 \mathrm{H}, \mathrm{CH}_{2}\right), 2.57(\mathrm{~d}, J=$ $\left.13.2 \mathrm{~Hz}, 1 \mathrm{H}, \mathrm{CH}_{2}\right), 1.50-1.20$ (br, $\left.18 \mathrm{H}\right), 1.07$ (br, $\left.3 \mathrm{H}\right)$.

\section{Imine 3b}

A solution of ${ }^{\mathrm{n}} \mathrm{BuLi}(2.5 \mathrm{M})$ in hexanes $(48 \mathrm{~mL}, 120 \mathrm{mmol})$ was added at $-78{ }^{\circ} \mathrm{C}$ to a stirred solution of DippN $=\mathrm{CHCH}_{2} \mathrm{CH}_{3} \mathbf{1}$ $(21.73 \mathrm{~g}, 100 \mathrm{mmol})$ in THF $(40 \mathrm{~mL})$. The solution was warmed to room temperature and stirred for $1 \mathrm{~h}$ and then cooled to $-78^{\circ} \mathrm{C}$.<smiles>C=C(C)CC(C)(C=Nc1ccccc1)CC(=C)C</smiles>
3-Chloro-2-methylpropene $(14.7 \mathrm{~mL}, 13.58 \mathrm{~g}, 150 \mathrm{mmol})$ was added in three portions. The solution was warmed to room temperature and stirred for $1 \mathrm{~h}$ and then cooled to $-78^{\circ} \mathrm{C}$. A solution of ${ }^{n} \mathrm{BuLi}(2.5 \mathrm{M})$ in hexanes $(60 \mathrm{~mL}, 150 \mathrm{mmol})$ was then added at $-78{ }^{\circ} \mathrm{C}$ and the solution was warmed to room temperature. After stirring for $1 \mathrm{~h}$, the solution was cooled to $-78{ }^{\circ} \mathrm{C}$ and 3-Chloro-2-methylpropene $(17.7 \mathrm{~mL}, 16.3 \mathrm{~g}, 180 \mathrm{mmol})$ was added in three portions. The solution was slowly warmed to room temperature overnight. Removal of the volatiles under vacuum afforded a mixture of an oil (alkenyl aldimine $\mathbf{3 b}$ ), as well as a solid $(\mathrm{LiCl})$. The crude product was directly used for the next step.

${ }^{1} \mathrm{H}$ NMR $\left(\mathrm{CDCl}_{3}, 400 \mathrm{MHz}, \mathrm{ppm}\right): \delta=7.68$ (s, 1H, DippN=CH), 7.15-7.05 (m, 3H, ArH), $4.93\left(\mathrm{~m}, 2 \mathrm{H},=\mathrm{CH}_{2}\right), 4.80\left(\mathrm{~m}, 2 \mathrm{H},=\mathrm{CH}_{2}\right), 2.97$ (sept, $\left.J=6.8 \mathrm{~Hz}, 2 \mathrm{H}, \mathrm{C} H \mathrm{Me}_{2}\right), 2.41$ (s, 3H, $\left.\mathrm{CH}_{3}\right), 1.82\left(\mathrm{~m}, 5 \mathrm{H}, \mathrm{CH}_{2}\right.$ and $\left.\mathrm{CH}_{3}\right), 1.36\left(\mathrm{~s}, 3 \mathrm{H}, \mathrm{CH}_{3}\right), 1.30-1.20\left(\mathrm{~s}, 2 \mathrm{H}, \mathrm{CH}_{2}\right), 1.16(\mathrm{~d}, J=6.8$ $\mathrm{Hz}, 12 \mathrm{H}, \mathrm{CHMe} 2) .{ }^{13} \mathrm{C}\left\{{ }^{1} \mathrm{H}\right\}$ NMR $\left(\mathrm{CDCl}_{3}, 125 \mathrm{MHz}, \mathrm{ppm}\right): \delta=173.0(\mathrm{CH}=\mathrm{NAr}), 148.6$, 142.5, 137.6, 123.9, 123.0, $115.5\left(\mathrm{Ar} C\right.$ and $\left.C=\mathrm{CH}_{2}\right), 46.4,27.5,25.4,23.7$.
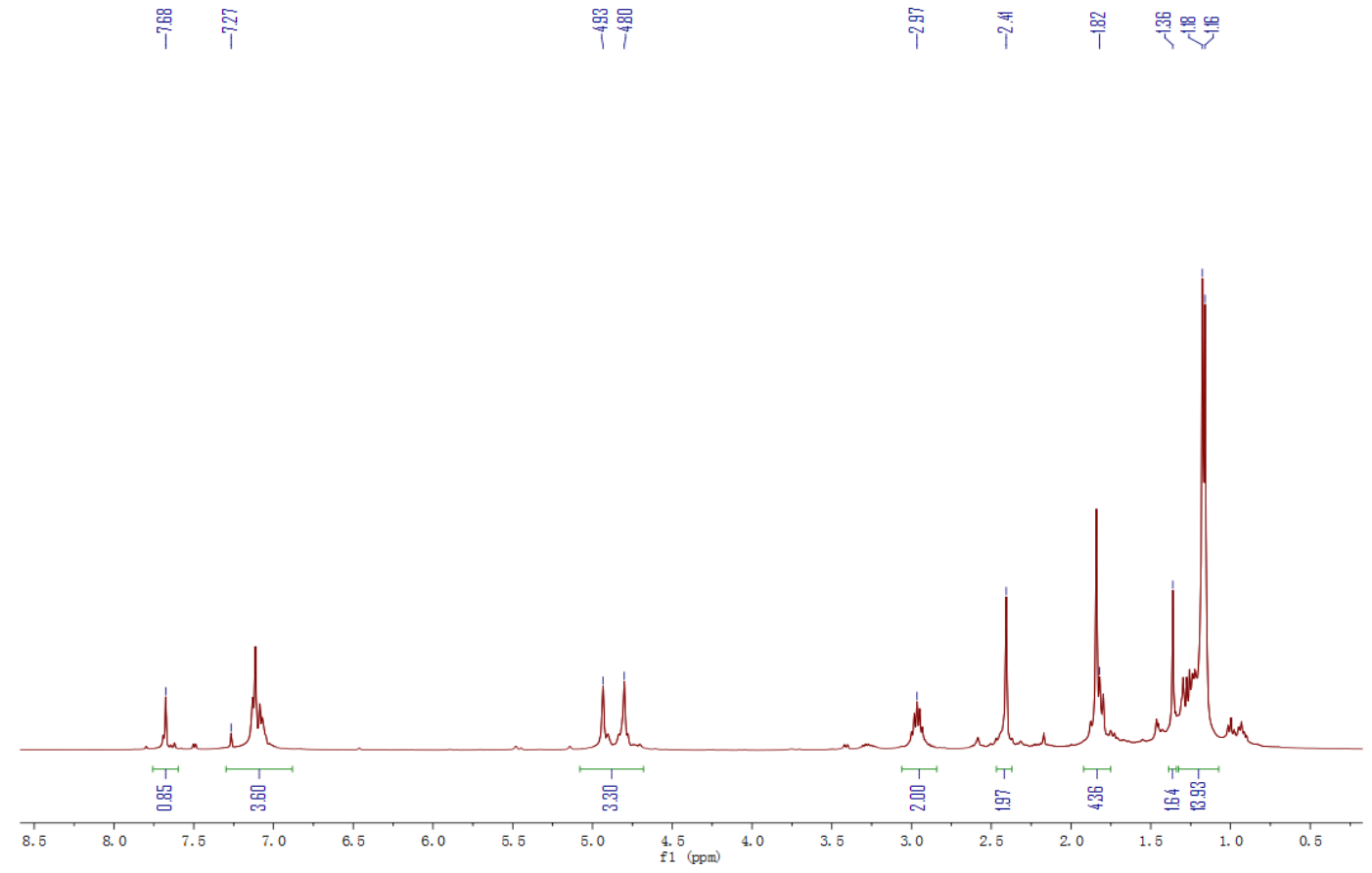

Figure S5. ${ }^{1} \mathrm{H}$ NMR of $\mathbf{3 b}\left(\mathrm{CDCl}_{3}, 400 \mathrm{MHz}\right)$. 


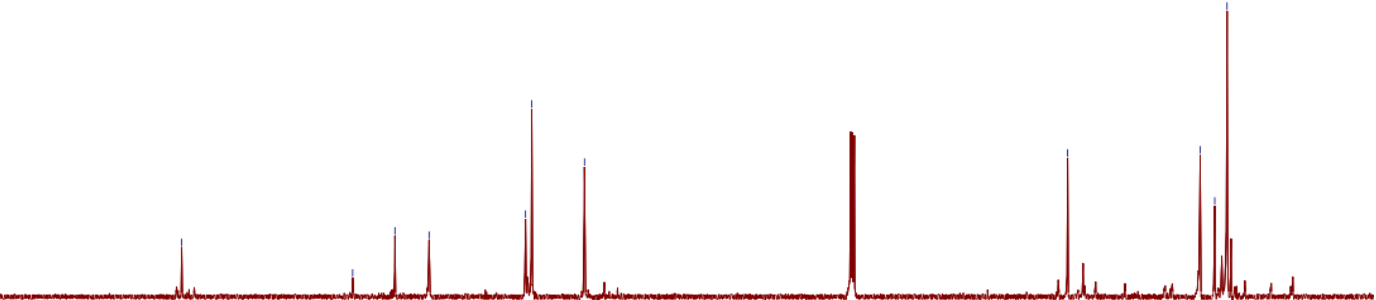

Figure S6. ${ }^{13} \mathrm{C}\left\{{ }^{1} \mathrm{H}\right\} \mathrm{NMR}$ of $\mathbf{3 b}\left(\mathrm{CDCl}_{3}, 125 \mathrm{MHz}\right)$.

\section{Imine 3c}

A solution of ${ }^{n} \operatorname{BuLi}(2.5 \mathrm{M})$ in hexanes $(53 \mathrm{~mL}, 132.5 \mathrm{mmol})$ was added at $-78{ }^{\circ} \mathrm{C}$ to a stirred solution of DippN $=\mathrm{CHCH}_{2} \mathrm{CH}_{3} 1(24 \mathrm{~g}$, $110.42 \mathrm{mmol})$ in THF $(40 \mathrm{~mL})$. The solution was warmed to room temperature and stirred for $1 \mathrm{~h}$ and then cooled to $-78{ }^{\circ} \mathrm{C}$. 3-Chloro-2-methylpropene $(16.3 \mathrm{~mL}, 15.0 \mathrm{~g}, 165 \mathrm{mmol})$ was added in<smiles>C=CCC(C)(C=Nc1ccccc1)CC(=C)C</smiles>
three portions. The solution was warmed to room temperature and stirred for $1 \mathrm{~h}$ and then cooled to $-78{ }^{\circ} \mathrm{C}$. A solution of ${ }^{n} \mathrm{BuLi}(2.5 \mathrm{M})$ in hexanes $(66.25 \mathrm{~mL}, 165.63 \mathrm{mmol})$ was added at $-78{ }^{\circ} \mathrm{C}$ to this solution. The solution was warmed to room temperature and stirred for $1 \mathrm{~h}$ and then cooled to $-78{ }^{\circ} \mathrm{C}$. 3-Chloropropene $(18.0 \mathrm{~mL}, 16.9 \mathrm{~g}, 220 \mathrm{mmol})$ was added in three portions. The solution was slowly warmed to room temperature overnight. Removal of the volatiles under vacuum afforded a mixture of an oil (alkenyl aldimine 3c) as well as a solid $(\mathrm{LiCl})$. The crude product was directly used for the next step

\section{Cyclic iminium salt 4a}

A solution of $\mathrm{HCl}$ in ether $(115 \mathrm{~mL}, 2.0 \mathrm{M}, 230 \mathrm{mmol})$ was added to a solution of crude alkenyl aldimine $3 \mathbf{a}(115 \mathrm{mmol})$ in diethylether $(100 \mathrm{~mL})$ at $-78{ }^{\circ} \mathrm{C}$. Precipitation of a colorless powder was immediately observed. After 15 minutes the mixture<smiles>CCOCC1(C)CC(C)(C)N([Pb])[C@H]1C(=O)OC</smiles>
was warmed to room temperature and stirring was continued for an additional 15 minutes. The solution was heated in a pressure tube at $110{ }^{\circ} \mathrm{C}$ for $24 \mathrm{~h}$ and then cooled to room temperature. The ether solution was removed and the solid was washed with diethylether until the solution was colorless. The solid was put under vacuum to afford a colorless powder, which was dissolved in water. $\mathrm{NaPF}_{6}(9 \mathrm{~g}, 233 \mathrm{mmol})$ was dissolved in water and added dropwise, upon which a precipitate formed. The precipitate was collected and triturated with 
$\mathrm{Et}_{2} \mathrm{O}$ and pentane. It was dried in vacuo and stirred over night with $\mathrm{K}_{3} \mathrm{PO}_{4}$ in $\mathrm{MeCN}$. Volatiles were evaporated and it was extracted with $\mathrm{CH}_{2} \mathrm{Cl}_{2}$. After washing with $\mathrm{Et}_{2} \mathrm{O}$ and drying in vacuo over night at $45^{\circ} \mathrm{C}$, an off white powder was obtained $(23.5 \mathrm{~g}, 43 \%$ based on 2,6-diisopropylaniline). m.p. $147^{\circ} \mathrm{C}$.

${ }^{1} \mathrm{H} \mathrm{NMR}\left(\mathrm{CDCl}_{3}, 400 \mathrm{MHz}, \mathrm{ppm}\right): \delta=8.82(\mathrm{~s}, 1 \mathrm{H}$, iminium- $H), 7.52(\mathrm{t}, J=7.7 \mathrm{~Hz}, 1 \mathrm{H}, \mathrm{p}-H)$, $7.34(\mathrm{~m}, 2 \mathrm{H}, \mathrm{m}-H), 4.12\left(\mathrm{~d}, 1 \mathrm{H}, J=8.8 \mathrm{~Hz}, \mathrm{CH}_{2}\right), 3.65\left(\mathrm{~m}, 1 \mathrm{H}, \mathrm{CH}_{2} \mathrm{Me}\right), 3.54(\mathrm{~m}, 1 \mathrm{H}$, $\left.\mathrm{CH}_{2} \mathrm{Me}\right), 3.44\left(\mathrm{~d}, J=8.8 \mathrm{~Hz}, 1 \mathrm{H}, \mathrm{CH}_{2}\right), 2.94\left(\mathrm{~m}, 1 \mathrm{H}, \mathrm{C} H \mathrm{Me}_{2}\right), 2.76(\mathrm{~d}, J=13.6 \mathrm{~Hz}, 1 \mathrm{H}$, $\mathrm{CH}_{2}$ ) $), 2.62\left(\mathrm{~m}, 1 \mathrm{H}, \mathrm{C} H \mathrm{Me}_{2}\right), 2.28\left(\mathrm{~d}, J=13.6 \mathrm{~Hz}, 1 \mathrm{H}, \mathrm{CH}_{2}\right), 1.57\left(\mathrm{~s}, 3 \mathrm{H}, \mathrm{CH}_{3}\right), 1.55(\mathrm{~s}, 3 \mathrm{H}$, $\left.\mathrm{CH}_{3}\right), 1.53\left(\mathrm{~s}, 3 \mathrm{H}, \mathrm{CH}_{3}\right), 1.35\left(\mathrm{~d}, J=7.0 \mathrm{~Hz}, 3 \mathrm{H}, \mathrm{CH}_{3}\right), 1.30\left(\mathrm{~d}, J=7.0 \mathrm{~Hz}, 3 \mathrm{H}, \mathrm{CH}_{3}\right), 1.21(\mathrm{t}$, $\left.J=7.0 \mathrm{~Hz}, 3 \mathrm{H}, \mathrm{CH}_{3}\right), 1.14\left(\mathrm{~d}, J=7.0 \mathrm{~Hz}, 3 \mathrm{H}, \mathrm{CH}_{3}\right), 1.10\left(\mathrm{~d}, J=7.0 \mathrm{~Hz}, 3 \mathrm{H}, \mathrm{CH}_{3}\right) .{ }^{13} \mathrm{C}\left\{{ }^{1} \mathrm{H}\right\}$ NMR $\left(\mathrm{CDCl}_{3}, 125 \mathrm{MHz}, \mathrm{ppm}\right): \delta=190.5$ (C-iminium), 144.9, 144.3, 131.9, 128.8, 125.5, $125.1(\mathrm{ArC}), 83.8\left(C_{\mathrm{q}}\right), 74.0\left(\mathrm{CH}_{2}\right), 67.0\left(\mathrm{CH}_{2}\right), 53.65,44.8\left(\mathrm{CH}_{2}\right), 29.8,29.0,28.8,27.7$, 26.4, 26.0, 22.0, 21.9, 21.1, 14.8. ${ }^{19} \mathrm{~F}\left(\mathrm{CDCl}_{3}, 376.4 \mathrm{MHz}, \mathrm{ppm}\right): \delta=-67.75(\mathrm{~d}, J=715.2 \mathrm{~Hz}$, $\left.\mathrm{PF}_{6}{ }^{-}\right)$. HRMS: $\mathrm{m} / \mathrm{z}$ calculated for $\left[\mathrm{C}_{22} \mathrm{H}_{36} \mathrm{NO}\right]^{+} 330.2791$, found 330.2792 .

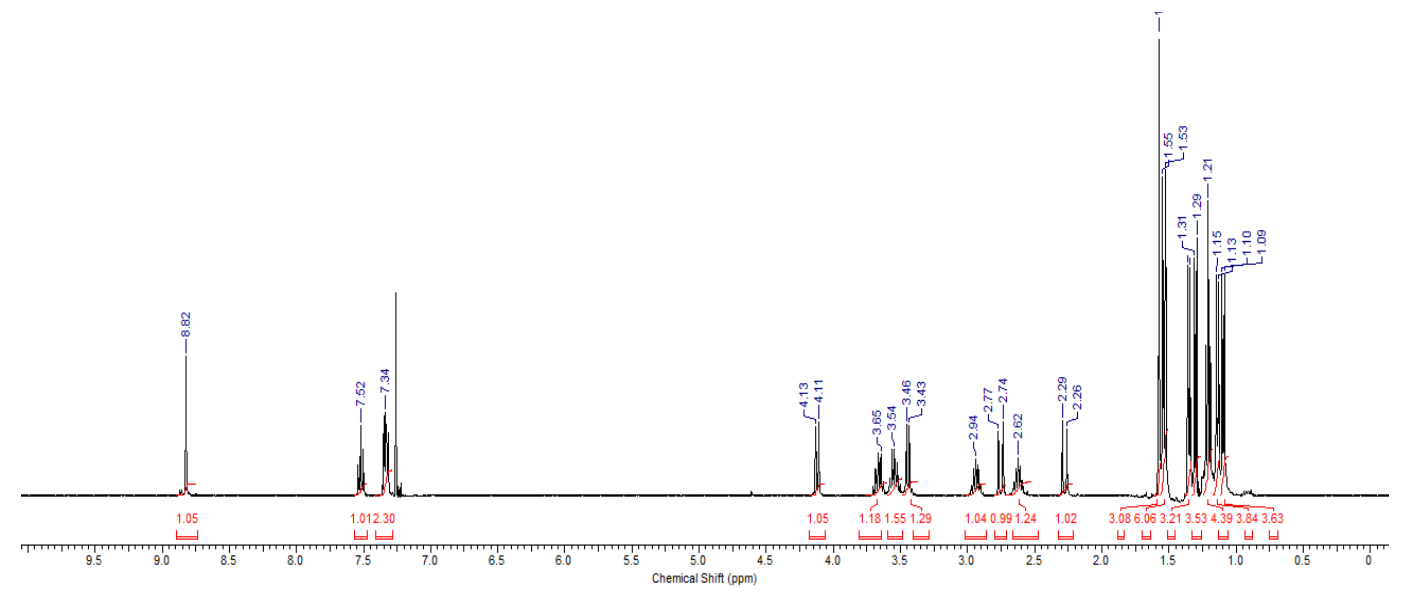

Figure S7. ${ }^{1} \mathrm{H}$ NMR of $4 \mathbf{a}\left(\mathrm{CDCl}_{3}, 400 \mathrm{MHz}\right)$.

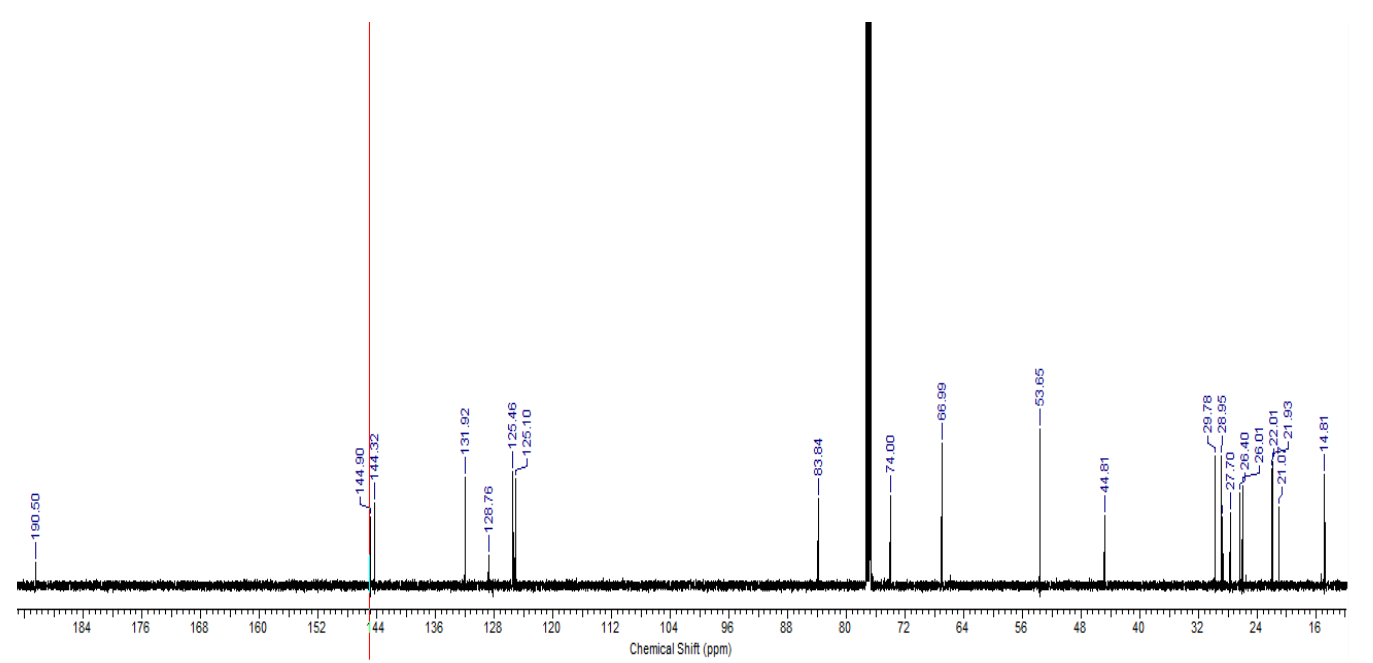

Figure S8. ${ }^{13} \mathrm{C}\left\{{ }^{1} \mathrm{H}\right\} \mathrm{NMR}$ of $\mathbf{4 a}\left(\mathrm{CDCl}_{3}, 125 \mathrm{MHz}\right)$. 


\section{Cyclic iminium salt $\mathbf{4 b}$}

A solution of $\mathrm{HCl}$ in ether $(100 \mathrm{~mL}, 2.0 \mathrm{M}, 200 \mathrm{mmol})$ was added to a solution of alkenyl aldimine $\mathbf{3 b}(100 \mathrm{mmol})$ in ether $(100 \mathrm{~mL})$ at $-78{ }^{\circ} \mathrm{C}$. Precipitation of a colorless powder was immediately observed. After 15 minutes the mixture was<smiles>C=C(C)CC1(C)CC(C)(C)N([Pb])[C@@H]1P</smiles>
warmed to room temperature and stirring was continued for an additional 15 minutes. The solution was heated in a pressure tube at $110^{\circ} \mathrm{C}$ for $24 \mathrm{~h}$ and then cooled to room temperature. The ether solution was removed and the solid was washed with ether until the ether solution was colorless. The solid was put under vacuum to afford a colorless powder. To a Schlenk tube containing the iminium salt $\left(\mathrm{X}=\mathrm{HCl}_{2}\right)$ and $\mathrm{NaBF}_{4}(21.96 \mathrm{~g}, 200 \mathrm{mmol})$ was added $\mathrm{H}_{2} \mathrm{O}$ $(200 \mathrm{~mL})$. The mixture was stirred for $1 \mathrm{~h}$ at room temperature and filtered. The solid was washed with $\mathrm{H}_{2} \mathrm{O}(2 \mathrm{x} 100 \mathrm{~mL})$, ether $(2 \mathrm{x}, 100 \mathrm{~mL})$, pentane $(2 \mathrm{x}, 100 \mathrm{~mL})$, and then dried under vacuum to get a colorless powder (19.6 g, 47\% based on 2,6-diisopropylaniline). m.p. $204{ }^{\circ} \mathrm{C}$.

${ }^{1} \mathrm{H}$ NMR $\left(\mathrm{CDCl}_{3}, 500 \mathrm{MHz}, \mathrm{ppm}\right): \delta=9.21(\mathrm{~s}, 1 \mathrm{H}$, iminium- $H), 7.51(\mathrm{t}, J=8.0 \mathrm{~Hz}, 1 \mathrm{H}, p-\mathrm{H})$, $\left.7.32(\mathrm{~m}, 2 \mathrm{H}, m-\mathrm{H}), 5.05\left(\mathrm{~s}, 1 \mathrm{H},=\mathrm{CH}_{2}\right), 4.97 \mathrm{~s}, \mathrm{sH},=\mathrm{CH}_{2}\right), 2.85\left(\mathrm{~d}, J=14.0 \mathrm{~Hz}, 1 \mathrm{H}, \mathrm{CH}_{2}\right)$, $2.66\left(\mathrm{~d}, J=14.0 \mathrm{~Hz}, 1 \mathrm{H}, \mathrm{CH}_{2}\right.$ ), 2.60 (sept, $J=7.0 \mathrm{~Hz}, 2 \mathrm{H}, \mathrm{C} H \mathrm{Me}_{2}$ ), $2.56(\mathrm{~d}, J=14.0 \mathrm{~Hz}, 2 \mathrm{H}$, $\left.\mathrm{CH}_{2}\right), 2.34\left(\mathrm{~d}, J=14.0 \mathrm{~Hz}, 2 \mathrm{H}, \mathrm{CH}_{2}\right), 1.84(\mathrm{~s}, 3 \mathrm{H}, \mathrm{CMe}), 1.68$ (s, 3H, CMe), 1.53 (s, 3H, CMe), 1.49 (s, 3H, CMe), 1.33 (d, J=6.8 Hz, 3H, CHMe $), 1.32$ (d, J=6.4 Hz, 3H, CHMe $)$, 1.17 (d, $J=7.0 \mathrm{~Hz}, 3 \mathrm{H}, \mathrm{CHMe}$ ), 1.10 (d, $\left.J=7.0 \mathrm{~Hz}, 3 \mathrm{H}, \mathrm{CHMe} e_{2}\right){ }^{13} \mathrm{C} \mathrm{NMR}\left(\mathrm{CDCl}_{3}, 125\right.$ MHz, ppm): $\delta=192.1$ (iminium $C$ ), 144.8, 144.5, 140.5, 132.0, 128.8, 125.5 (2 C), 117.9 $(\mathrm{ArC}), 83.5\left(\mathrm{C}_{\mathrm{q}}\right), 52.9\left(\mathrm{CH}_{2}\right), 45.4\left(\mathrm{CH}_{3}\right), 45.3\left(\mathrm{CH}_{3}\right), 29.9,29.8,28.8,28.1,26.7,26.1,26.0$, 24.9, 22.4. ${ }^{19} \mathrm{~F}$ NMR $\left(\mathrm{CD}_{3} \mathrm{CN}, 282 \mathrm{MHz}, \mathrm{ppm}\right): \delta=-155.4$. HRMS: $\mathrm{m} / \mathrm{z}$ calculated for $\left[\mathrm{C}_{23}\right.$ $\left.\mathrm{H}_{56} \mathrm{~N}\right]^{+} 326.2842$ found 326.2838 .
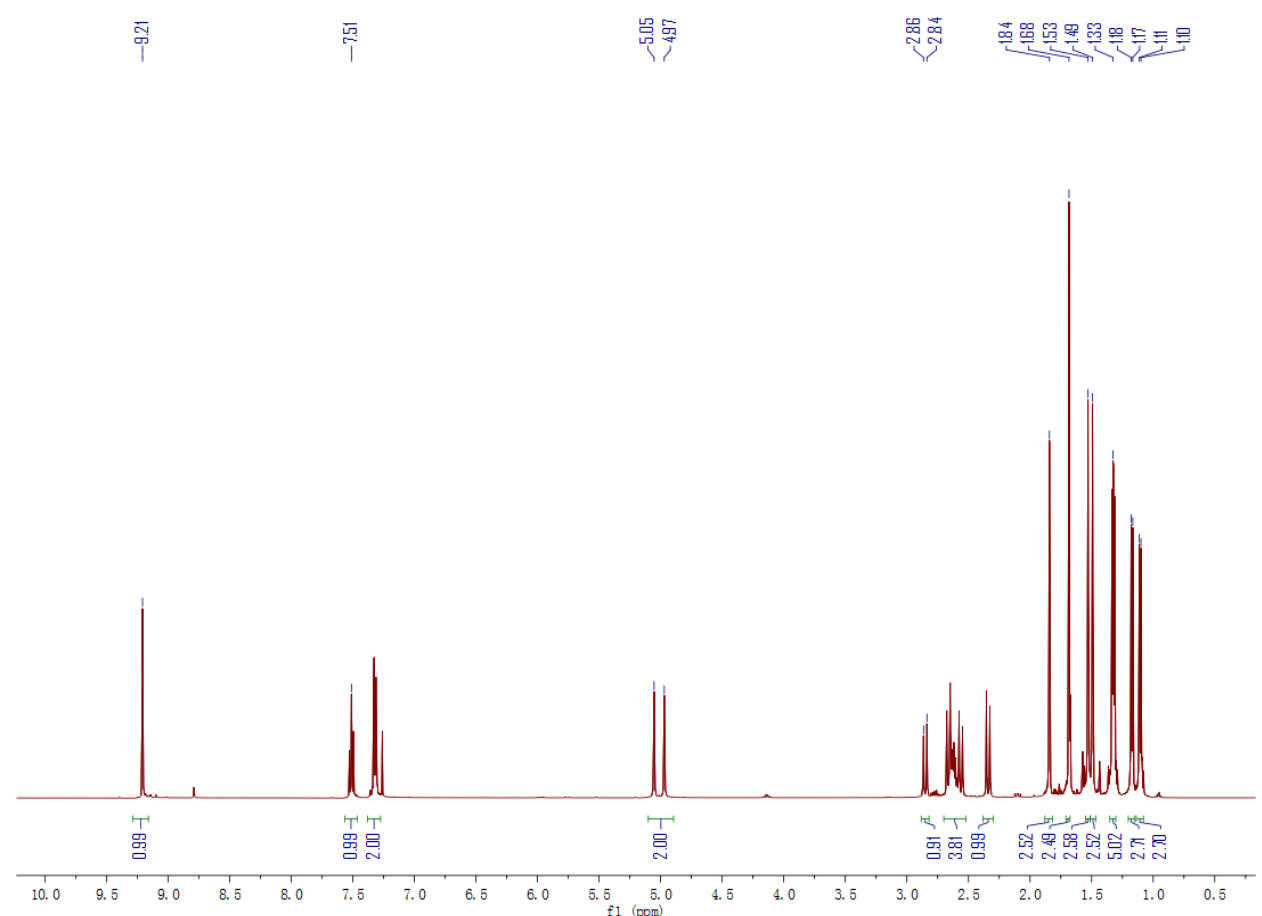

Figure S9. ${ }^{1} \mathrm{H} \mathrm{NMR}$ of $\mathbf{4 b}\left(\mathrm{CDCl}_{3}, 500 \mathrm{MHz}\right)$. 

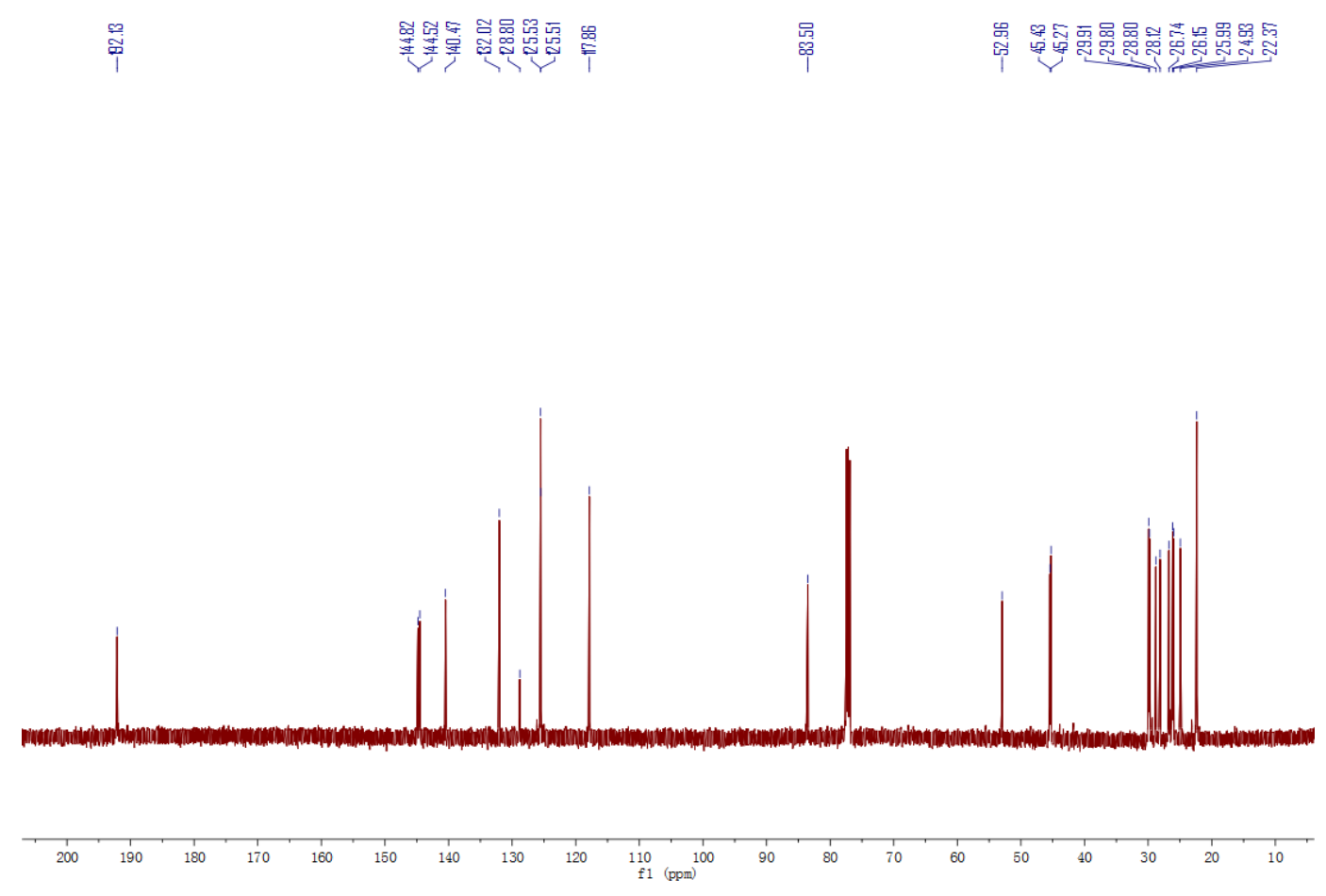

Figure S10. ${ }^{13} \mathrm{C}\left\{{ }^{1} \mathrm{H}\right\}$ NMR of $\mathbf{4 b}\left(\mathrm{CDCl}_{3}, 125 \mathrm{MHz}\right)$.

\section{Allyl functionalized cyclic iminiumsalt 4c}

A solution of $\mathrm{HCl}$ in ether $(110 \mathrm{~mL}, 2.0 \mathrm{M}, 220 \mathrm{mmol})$ was added to a solution of alkenyl aldimine $3 \mathbf{c}(110 \mathrm{mmol})$ in diethylether $(30 \mathrm{~mL})$ at $-78{ }^{\circ} \mathrm{C}$. Precipitation of a colorless powder was immediately

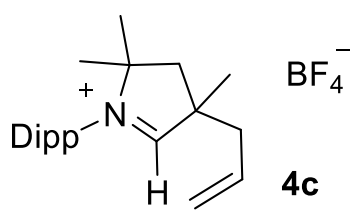
observed. The mixture was warmed to room temperature after $15 \mathrm{~min}$ and stirring was continued for an additional 15 minutes. The solution was heated in a pressure tube at $110{ }^{\circ} \mathrm{C}$ for $24 \mathrm{~h}$ and then cooled to room temperature. The ether solution was removed and the solid was washed with ether until the ether solution was colorless. The solid was put under vacuum to afford a colorless powder. $\mathrm{H}_{2} \mathrm{O}(200 \mathrm{~mL})$ was added to a Schlenk tube containing the iminium salt $\left(\mathrm{X}=\mathrm{HCl}_{2}{ }^{-}\right)$and $\mathrm{NaBF}_{4}(24.24 \mathrm{~g}, 220 \mathrm{mmol})$. The mixture was stirred for $1 \mathrm{~h}$ at room temperature and filtered. The solid was washed with $\mathrm{H}_{2} \mathrm{O}(100 \mathrm{~mL}, 2 \mathrm{x})$, ether $(100 \mathrm{~mL}$, $2 \mathrm{x})$, pentane $(100 \mathrm{~mL}, 2 \mathrm{x})$, and then dried under vacuum to get a colorless powder $(15.7 \mathrm{~g}, 36 \%$ based on 2,6-diisopropylaniline). m.p. $233^{\circ} \mathrm{C}$.

${ }^{1} \mathrm{H}$ NMR $\left(\mathrm{CDCl}_{3}, 500 \mathrm{MHz}, \mathrm{ppm}\right): \delta=9.29(\mathrm{~s}, 1 \mathrm{H}$, iminium- $H), 7.51(\mathrm{t}, J=7.5 \mathrm{~Hz}, 1 \mathrm{H}, p-\mathrm{H})$, $7.33(\mathrm{~d}, J=7.5 \mathrm{~Hz}, 2 \mathrm{H}, m-\mathrm{H}), 5.84$ (ddt, $J=16.6 \mathrm{~Hz}, 10.2 \mathrm{~Hz}, 7.3 \mathrm{~Hz}, 1 \mathrm{H}, \mathrm{CH}_{2}=\mathrm{CHCH}_{2}$ ), $5.40\left(\mathrm{~d}, J=16.6 \mathrm{~Hz}, 1 \mathrm{H},=\mathrm{CH}_{2}\right), 5.29\left(\mathrm{~d}, J=10.2 \mathrm{~Hz}, 1 \mathrm{H},=\mathrm{CH}_{2}\right), 2.97(\mathrm{dd}, J=13.7 \mathrm{~Hz}, J=$ $7.3 \mathrm{~Hz}, 1 \mathrm{H}, \mathrm{CH}_{2}$ ), 2.65 (sept, $J=6.8 \mathrm{~Hz}, 1 \mathrm{H}, \mathrm{C} H \mathrm{Me}_{2}$ ), 2.62 (sept, $J=6.8 \mathrm{~Hz}$, $\left.1 \mathrm{H}, \mathrm{C} H \mathrm{Me}_{2}\right), 2.57\left(\mathrm{~d}, J=14.0 \mathrm{~Hz}, 1 \mathrm{H}, \mathrm{CH}_{2}\right), 2.52\left(\mathrm{dd}, J=13.7 \mathrm{~Hz}, J=7.3 \mathrm{~Hz}, 1 \mathrm{H}, \mathrm{CH}_{2}\right), 2.30$ (d, $\left.J=14.0 \mathrm{~Hz}, 1 \mathrm{H}, \mathrm{CH}_{2}\right), 1.71(\mathrm{~s}, 3 \mathrm{H}, \mathrm{CMe}), 1.55$ (s, 3H, CMe), 1.48 (s, 3H, CMe), 1.33 (d, $J=6.5 \mathrm{~Hz}, 3 \mathrm{H}, \mathrm{CHMe}$ ), 1.32 (d, $J=6.5 \mathrm{~Hz}, 3 \mathrm{H}, \mathrm{CHMe}$ ), 1.17 (d, $J=6.5 \mathrm{~Hz}, 3 \mathrm{H}, \mathrm{CHMe}$ ), $1.11(\mathrm{~d}, J=6.5 \mathrm{~Hz}, 3 \mathrm{H}, \mathrm{CHMe} 2) .{ }^{13} \mathrm{C}\left\{{ }^{1} \mathrm{H}\right\} \mathrm{NMR}\left(\mathrm{CDCl}_{3}, 125 \mathrm{MHz}, \mathrm{ppm}\right): \delta=192.7$ (iminium $C$ ), 144.6, 144.5, 132.1, 131.9, 128.9, 125.5, 125.4, $122.1(\operatorname{ArC}), 83.4\left(\mathrm{C}_{\mathrm{q}}\right), 52.9$ $\left(\mathrm{CH}_{2}\right), 45.3\left(\mathrm{CH}_{3}\right), 42.2\left(\mathrm{CH}_{3}\right), 29.9,29.8,28.7,28.1,26.5,26.2,25.6,22.4,22.1 .{ }^{19} \mathrm{~F}$ NMR 
$\left(\mathrm{CD}_{3} \mathrm{CN}, 282 \mathrm{MHz}, \mathrm{ppm}\right): \delta=-155.4$. HRMS: $\mathrm{m} / \mathrm{z}$ calculated for $\left[\mathrm{C}_{22} \mathrm{H}_{34} \mathrm{~N}\right]^{+}$312.2686, found 312.2683 .

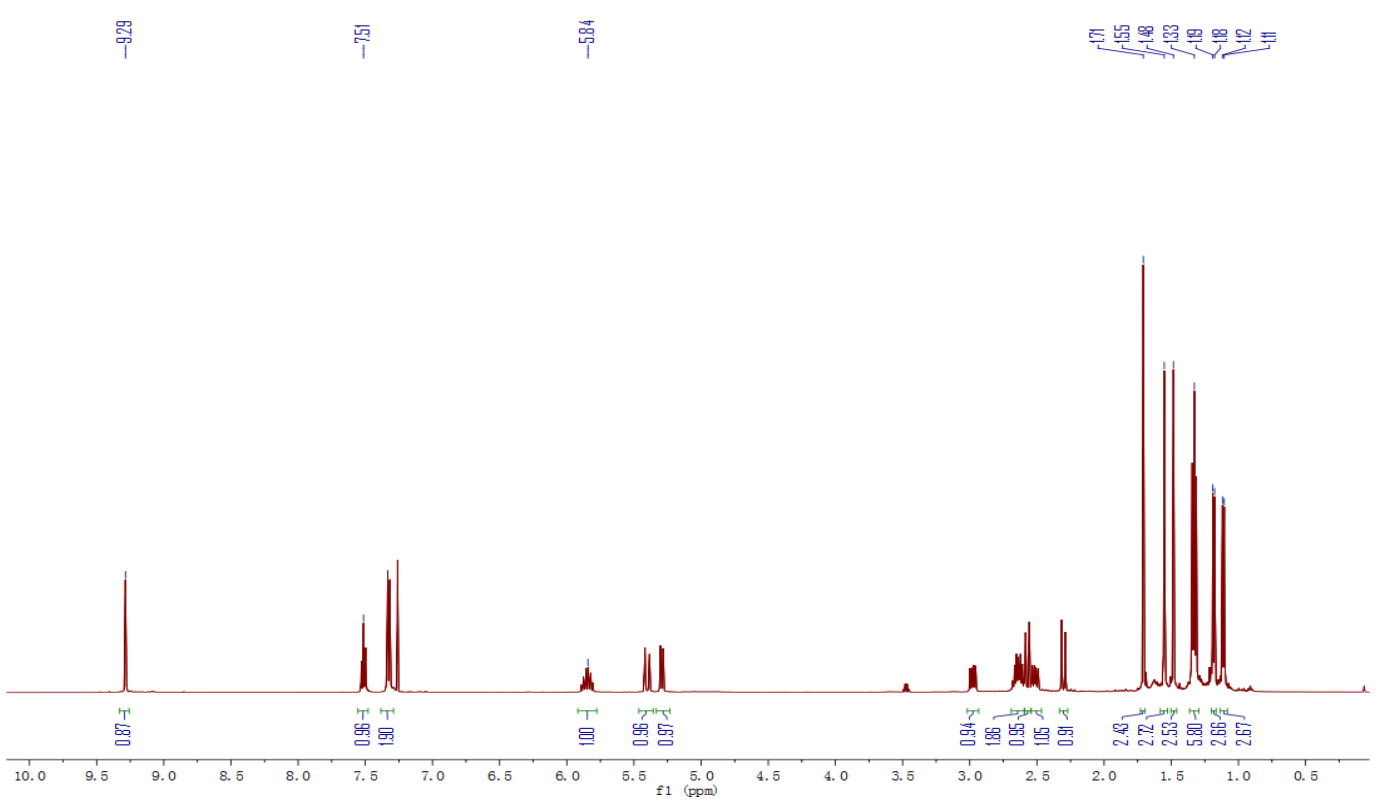

Figure S11. ${ }^{1} \mathrm{H}$ NMR of $4 \mathbf{c}\left(\mathrm{CDCl}_{3}, 500 \mathrm{MHz}\right)$.

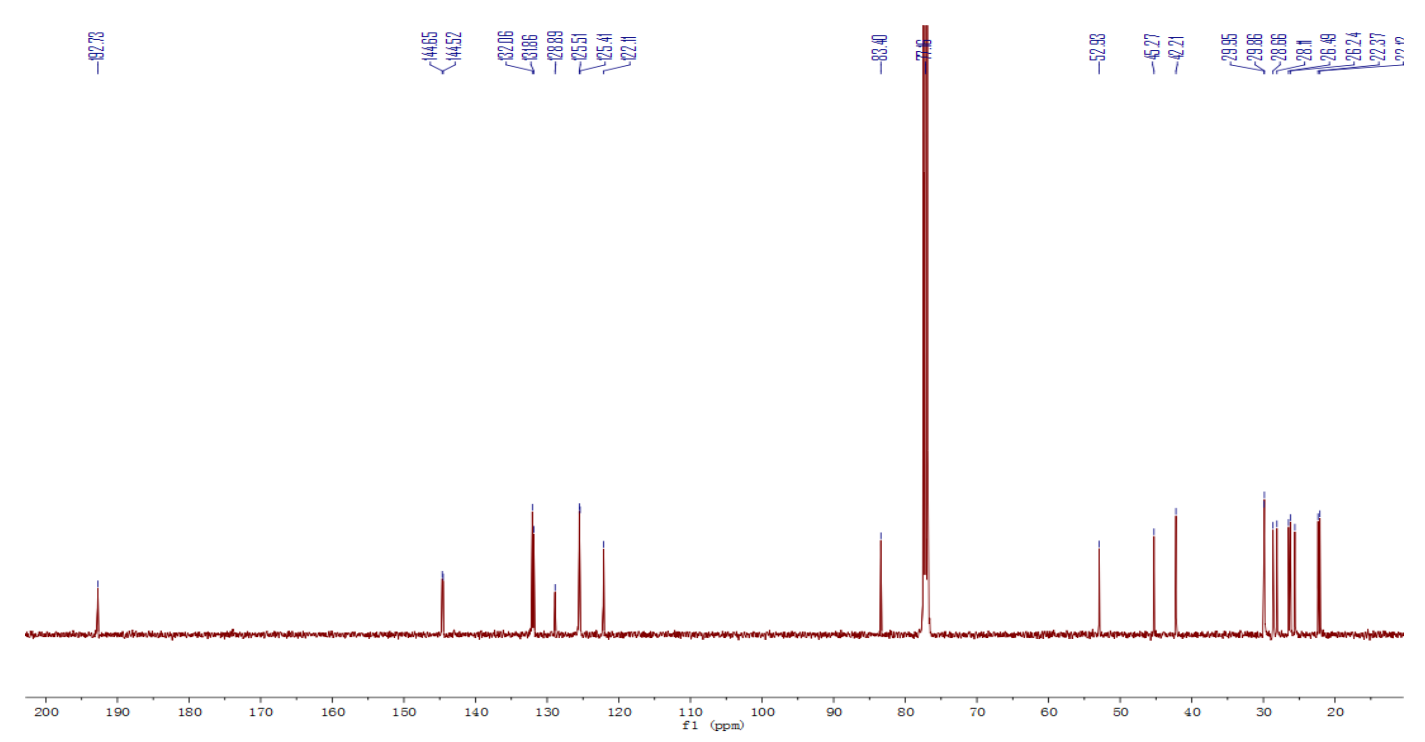

Figure S12. ${ }^{13} \mathrm{C}\left\{{ }^{1} \mathrm{H}\right\}$ NMR of $4 \mathbf{c}\left(\mathrm{CDCl}_{3}, 125 \mathrm{MHz}\right)$.

\section{Cylic iminium salt 4d}

$\mathrm{CH}_{2} \mathrm{Cl}_{2}(20 \mathrm{~mL})$ was added to a mixture of $\mathrm{Me}_{2} \mathrm{NCH}_{2} \mathrm{Cl}(1.40 \mathrm{~g}$, $15.0 \mathrm{mmol})$ and $\mathrm{NaBF}_{4}(1.65 \mathrm{~g}, 15.0 \mathrm{mmol})$, and the reaction mixture was stirred for $12 \mathrm{~h}$ at room temperature. The $\mathrm{CH}_{2} \mathrm{Cl}_{2}$ solution was filtered and the colorless solid was further washed

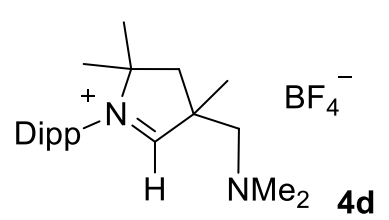
with $\mathrm{CH}_{2} \mathrm{Cl}_{2}(20 \mathrm{~mL})$. Enamine $5(3.26 \mathrm{~g}, 12.0 \mathrm{mmol})$ in $\mathrm{CH}_{2} \mathrm{Cl}_{2}(20 \mathrm{~mL})$ was added to the colorless solid and the reaction mixture was stirred for further $12 \mathrm{~h}$ at room temperature. The 
$\mathrm{CH}_{2} \mathrm{Cl}_{2}$ solution was filtered to another Schlenk flask and the solvent was removed in vacuo. The crude product was washed with ether $(2 \times 20 \mathrm{~mL})$ to afford a colorless powder $(4.5 \mathrm{~g}, 90 \%$ based on enamine, $46 \%$ based on 2,6-diisopropylaniline). m.p. $156-157{ }^{\circ} \mathrm{C}$ (dec.).

${ }^{1} \mathrm{H} \mathrm{NMR}\left(\mathrm{CDCl}_{3}, 500 \mathrm{MHz}, \mathrm{ppm}\right): \delta=9.19(\mathrm{~s}, 1 \mathrm{H}$, iminium- $H), 7.52(\mathrm{t}, J=7.6 \mathrm{~Hz}, 1 \mathrm{H}, p-\mathrm{H})$, 7.32-7.34 (m, 2H, $m-\mathrm{H}), 3.31(\mathrm{~d}, J=14.6 \mathrm{~Hz}, 1 \mathrm{H}, \mathrm{CH}), 2.92\left(\mathrm{~d}, J=6.4 \mathrm{~Hz}, 1 \mathrm{H}, \mathrm{C} H \mathrm{Me}_{2}\right)$, $2.77\left(\mathrm{~d}, J=13.2 \mathrm{~Hz}, 1 \mathrm{H}, \mathrm{CH}_{2} \mathrm{NMe}_{2}\right), 2.63\left(\mathrm{~d}, J=6.9 \mathrm{~Hz}, 1 \mathrm{H}, \mathrm{C} H \mathrm{Me}_{2}\right), 2.58(\mathrm{~d}, J=14.6 \mathrm{~Hz}$, $\left.1 \mathrm{H}, \mathrm{CH}_{2}\right), 2.41\left(\mathrm{~s}, 6 \mathrm{H}, \mathrm{NMe}\right.$ ) $, 2.26\left(\mathrm{~d}, J=13.2 \mathrm{~Hz}, 1 \mathrm{H}, \mathrm{CH}_{2} \mathrm{NMe}_{2}\right), 1.57$ (s, 3H, CMe), 1.56 (s, 3H, CMe $), 1.53$ (s, 3H, CMe $), 1.35$ (d, J=6.4 Hz, 6H, CHMe $), 1.34$ (d, J=6.9 Hz, 6H, $\left.\mathrm{CHMe}_{2}\right), 1.18\left(\mathrm{~d}, J=6.4 \mathrm{~Hz}, 6 \mathrm{H}, \mathrm{CHMe} e_{2}\right), 1.14(\mathrm{~d}, J=6.9 \mathrm{~Hz}, 6 \mathrm{H}, \mathrm{CHMe} 2) .{ }^{13} \mathrm{C}\left\{{ }^{1} \mathrm{H}\right\} \mathrm{NMR}$ $\left(\mathrm{CDCl}_{3}, 125 \mathrm{MHz}, \mathrm{ppm}\right): \delta=191.9$ (iminium $C$ ), 144.9, 144.8, 131.9, 129.1, 125.5, 125.3 $(\operatorname{ArC}), 83.3\left(C_{\mathrm{q}}\right), 66.4\left(\mathrm{CH}_{2}\right), 55.7\left(C_{\mathrm{q}}\right), 48.6\left(\mathrm{NMe}_{2}\right), 45.2\left(\mathrm{CH}_{2}\right), 29.9,29.6,28.5,28.4,27.3$, 26.2, 23.6, 22.6, 22.3. ${ }^{19} \mathrm{~F} \mathrm{NMR}\left(\mathrm{CD}_{3} \mathrm{CN}, 282 \mathrm{MHz}, \mathrm{ppm}\right): \delta=-155.4$. HRMS: $\mathrm{m} / \mathrm{z}$ calculated for $\left[\mathrm{C}_{22} \mathrm{H}_{37} \mathrm{~N}_{2}\right]^{+}$329.2951, found 329.2947.

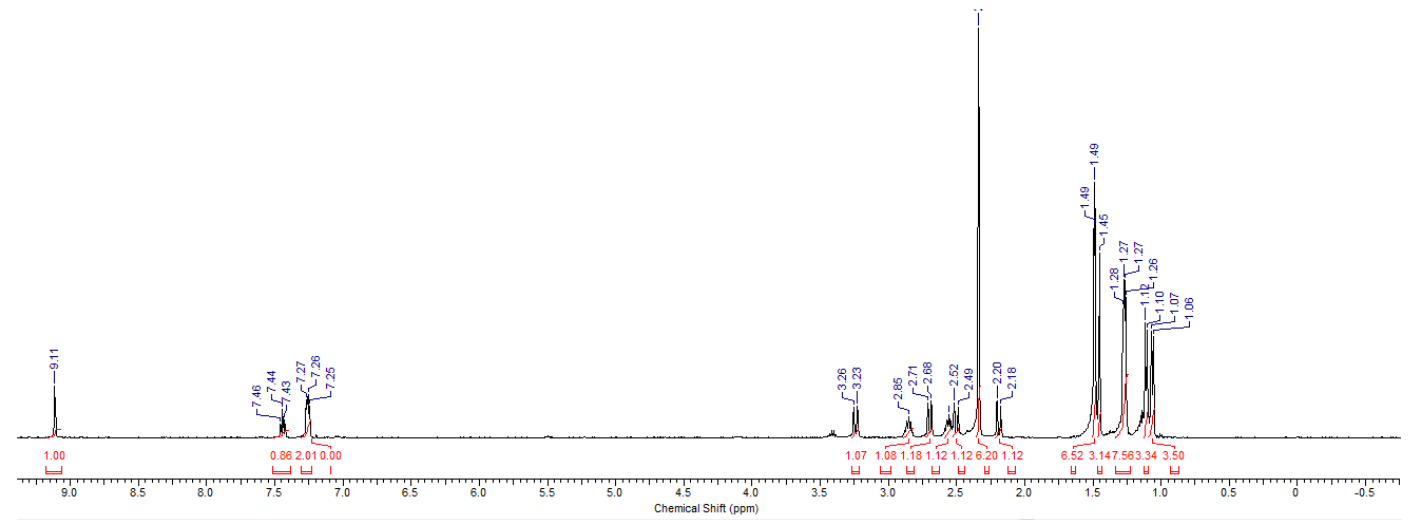

Figure S13. ${ }^{1} \mathrm{H}$ NMR of $\mathbf{4 d}\left(\mathrm{CDCl}_{3}, 500 \mathrm{MHz}\right)$.

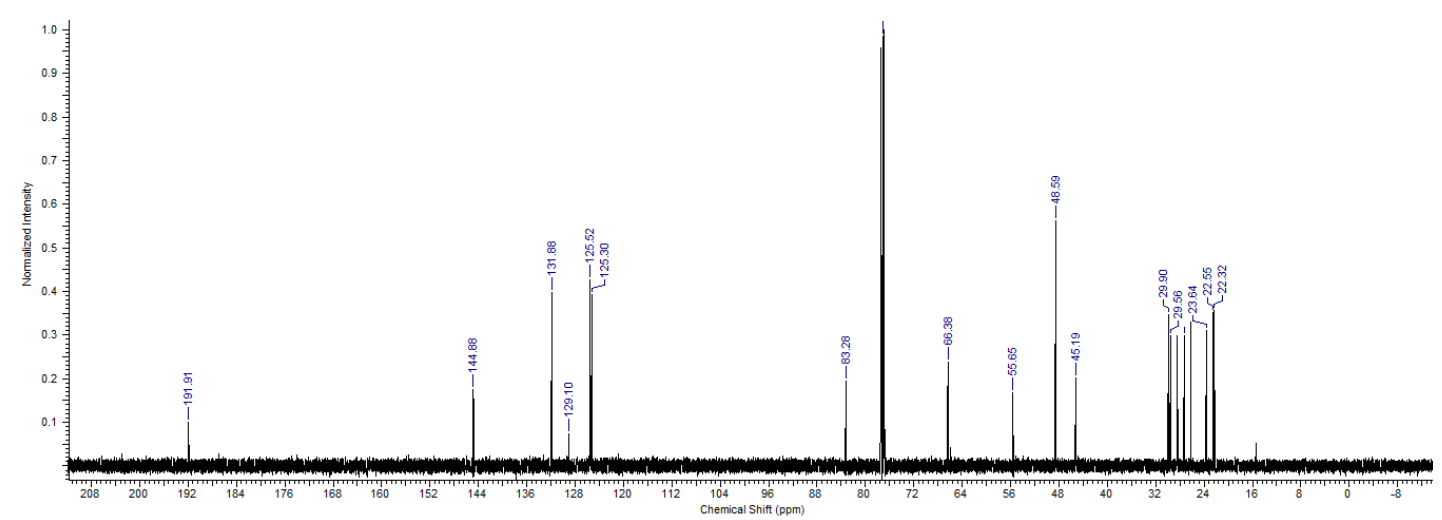

Figure S14. ${ }^{13} \mathrm{C}\left\{{ }^{1} \mathrm{H}\right\} \mathrm{NMR}$ of $\mathbf{4 d}\left(\mathrm{CD}_{3} \mathrm{CN}, 125 \mathrm{MHz}\right)$.

\section{Cyclic iminium salt 4e}

$\mathrm{CH}_{3} \mathrm{CN}(15 \mathrm{~mL})$ was added to a mixture of $\mathrm{NaBF}_{4}(2.41 \mathrm{~g}$, $22.0 \mathrm{mmol}), \mathrm{N}$-diisopropylphenylpivalimidoyl chloride (5.60 g, $20.0 \mathrm{mmol})$, and enamine 5 (5.43 g, $20 \mathrm{mmol})$. After the reaction mixture was stirred for $12 \mathrm{~h}$ at room temperature, $\mathrm{K}_{3} \mathrm{PO}_{4}(2.12 \mathrm{~g}, 10 \mathrm{mmol})$ was added and the reaction mixture

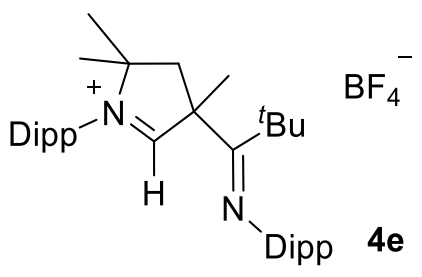
was stirred for another $12 \mathrm{~h}$. The $\mathrm{CH}_{3} \mathrm{CN}$ solution was filtered and the solvent was removed 
in vacuo. The crude product was extracted with $\mathrm{CH}_{2} \mathrm{Cl}_{2}(120 \mathrm{~mL})$. After $\mathrm{CH}_{2} \mathrm{Cl}_{2}$ was removed in vacuo, the pale yellow solid was washed with hexane $(100 \mathrm{~mL})$ to afford a pale yellow powder (10.85 g, 90\% based on enamine, $46 \%$ based on 2,6-diisopropylaniline). m.p. $215-217^{\circ} \mathrm{C}$ (dec.).

${ }^{1} \mathrm{H} \mathrm{NMR}\left(\mathrm{CDCl}_{3}, 300 \mathrm{MHz}, \mathrm{ppm}\right): \delta=8.47(\mathrm{~s}, 1 \mathrm{H}$, iminium- $H), 7.51(\mathrm{t}, J=7.8 \mathrm{~Hz}, 1 \mathrm{H}, p-\mathrm{H})$, $7.40(\mathrm{~m}, 1 \mathrm{H}, m-\mathrm{H}), 7.25(\mathrm{~m}, 1 \mathrm{H}, m-\mathrm{H}), 7.04(\mathrm{~s}, 3 \mathrm{H}, \mathrm{Ar} H), 3.17\left(\mathrm{~d}, J=13.5 \mathrm{~Hz}, 1 \mathrm{H}, \mathrm{CH}_{2}\right)$, 2.90-2.70 (m, 5H, $\mathrm{CHMe}$ and $\mathrm{CH}_{2}$ ), 2.25 (s, 3H, CMe), 1.70 (s, 3H, CMe), 1.57 (s, 3H, CMe), $1.37(\mathrm{~d}, J=6.6 \mathrm{~Hz}, 3 \mathrm{H}, \mathrm{CHMe}$ ), $1.31(\mathrm{~d}, J=6.6 \mathrm{~Hz}, 3 \mathrm{H}, \mathrm{CHMe}), 1.29$ (d, $J=6.6 \mathrm{~Hz}, 3 \mathrm{H}$, $\mathrm{CHMe}_{2}$ ), 1.25-1.15 (m, 18H, $\mathrm{CMe}_{3}$ and $\left.\mathrm{CHMe} e_{2}\right), 0.99$ (d, $J=6.6 \mathrm{~Hz}, 3 \mathrm{H}, \mathrm{CHMe}$ ), 0.68 (d, $J$ $=6.6 \mathrm{~Hz}, 3 \mathrm{H}, \mathrm{CHMe}) .{ }^{13} \mathrm{C}\left\{{ }^{1} \mathrm{H}\right\} \mathrm{NMR}\left(\mathrm{CDCl}_{3}, 125 \mathrm{MHz}, \mathrm{ppm}\right): \delta=183.7$ (iminium $C$ ), 182.7 (imine $C$ ), 145.8, 145.6, 143.6, 135.0, 134.6, 132.1, 128.8, 125.9, 125.5, 124.4, 123.4, 123.0 ( $\mathrm{ArC}), 83.2\left(C_{\mathrm{q}}\right), 64.7\left(C_{\mathrm{q}}\right), 50.0\left(\mathrm{CH}_{2}\right), 43.7\left(C_{\mathrm{q}}\right), 29.9\left(\mathrm{CMe}_{3}\right), 29.6,29.2,29.0,28.7$, 28.1, 27.7, 26.8, 26.4, 24.9, 24.8, 24.0, 23.1, 22.6, 22.4, 22.1. ${ }^{19} \mathrm{~F}$ NMR $\left(\mathrm{CD}_{3} \mathrm{CN}, 282 \mathrm{MHz}\right.$, ppm): $\delta=-155.4$. HRMS: $\mathrm{m} / \mathrm{z}$ calculated for $\left[\mathrm{C}_{36} \mathrm{H}_{55} \mathrm{~N}_{2}\right]^{+} 515.4360$, found 515.4363 .

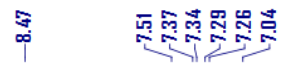

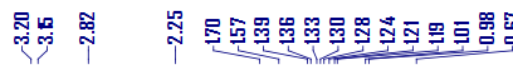

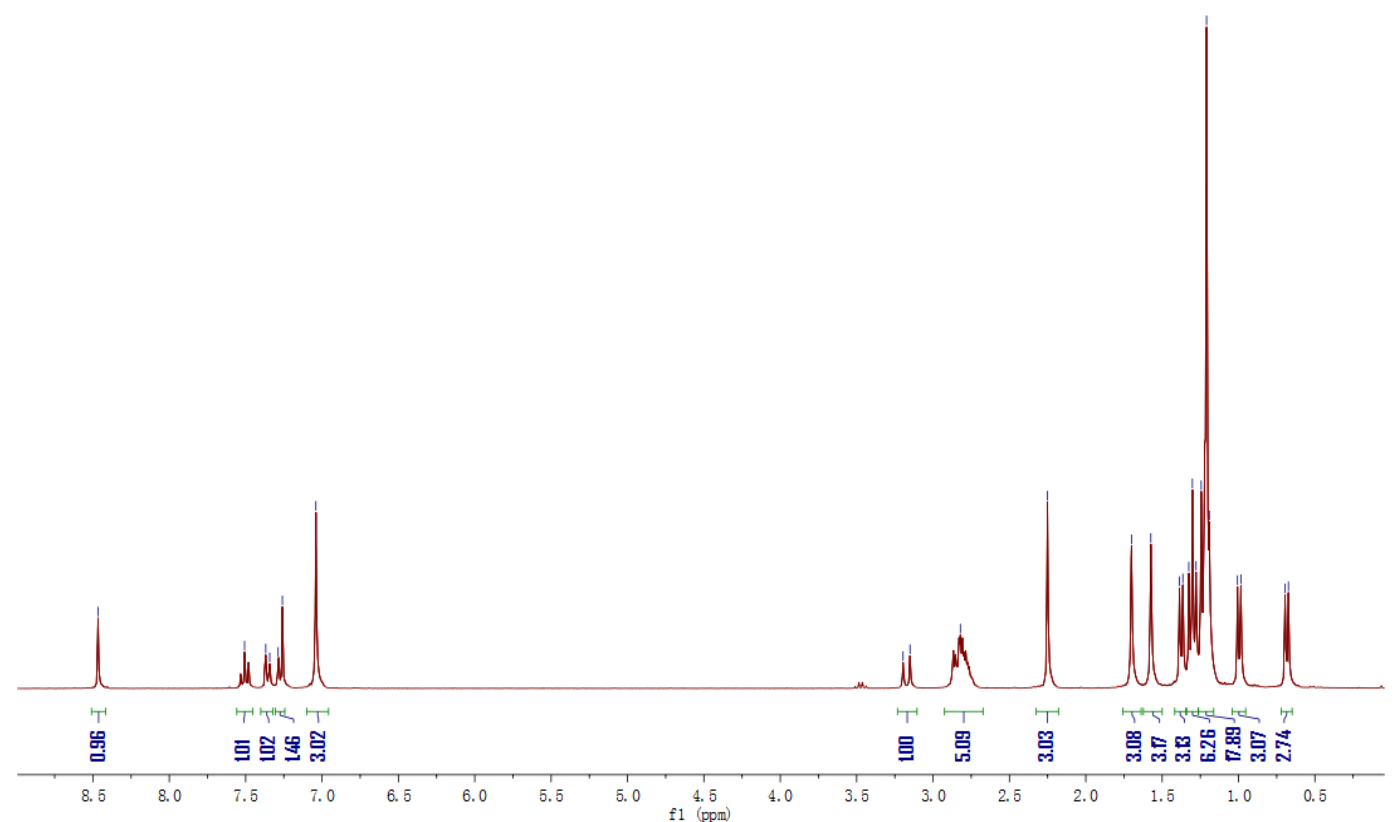

Figure S15. ${ }^{1} \mathrm{H}$ NMR of $4 \mathbf{e}\left(\mathrm{CDCl}_{3}, 300 \mathrm{MHz}\right)$. 


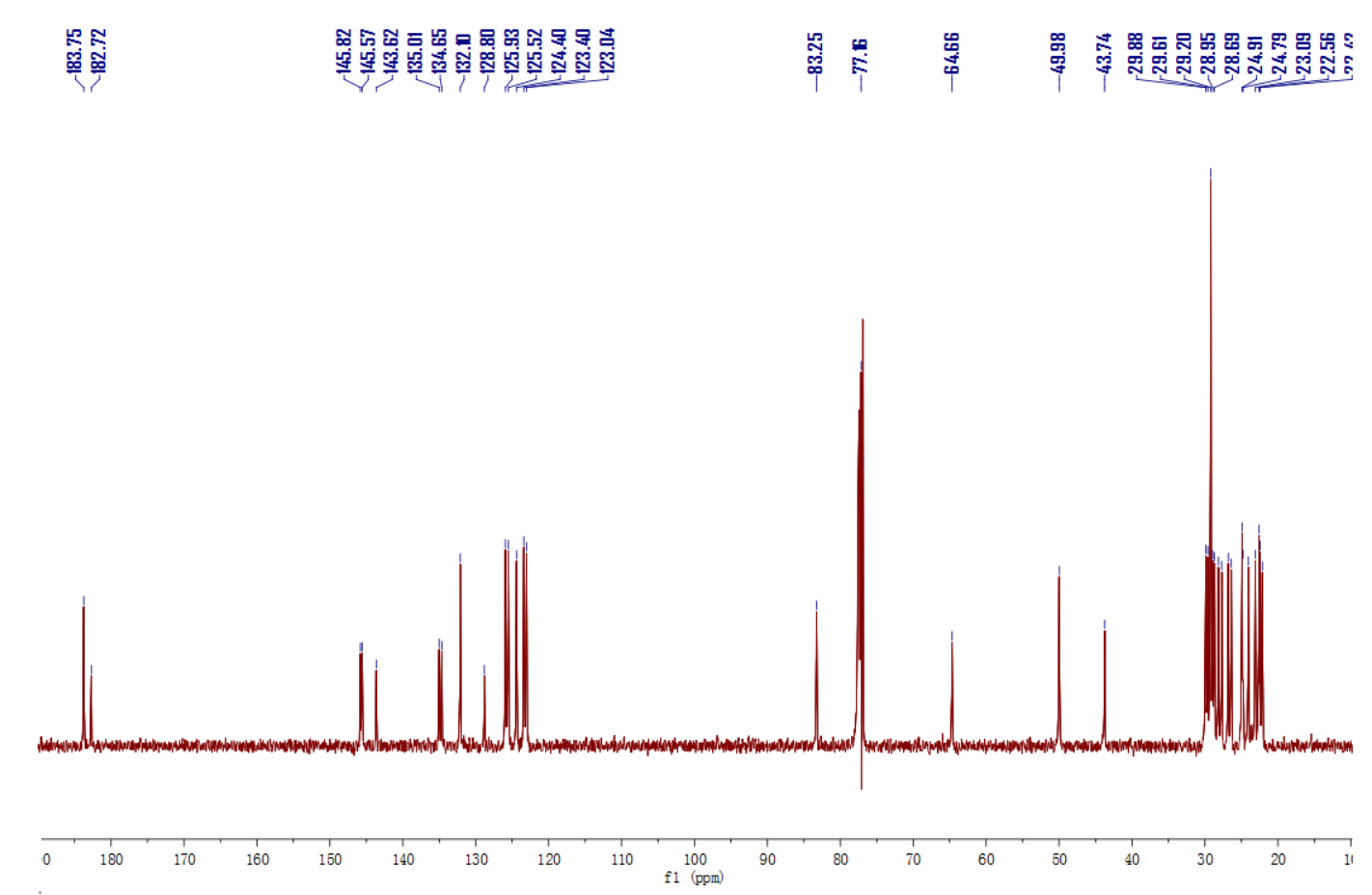

Figure S16. ${ }^{13} \mathrm{C}\left\{{ }^{1} \mathrm{H}\right\}$ NMR of $4 \mathbf{e}\left(\mathrm{CDCl}_{3}, 125 \mathrm{MHz}\right)$.

\section{Cyclic iminium salt $\mathbf{4 f}$}

$\mathrm{CH}_{2} \mathrm{I}_{2}(5.9 \mathrm{~mL}, 19.73 \mathrm{~g}, 74 \mathrm{mmol})$ was added to enamine 5 (2.40 g, $8.84 \mathrm{mmol}$ ) under Ar. The mixture was degassed three times, and then stirred at $100{ }^{\circ} \mathrm{C}$ for $12 \mathrm{~h}$. The reaction solution was cooled to room temperature, and the precipitate was washed with diethylether until the

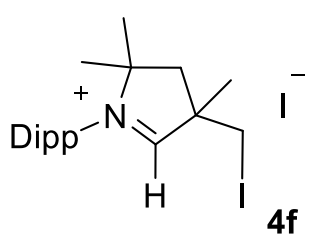
ether solution was nearly colorless. The remaining yellowish solid was dried at $100{ }^{\circ} \mathrm{C}$ under high vacuum for 12 hour ( $4.2 \mathrm{~g}, 88 \%$ based on enamine, $45 \%$ based 2,6-diisopropylaniline). m.p. $246^{\circ} \mathrm{C}$.

${ }^{1} \mathrm{H}$ NMR $\left(\mathrm{CDCl}_{3}, 500 \mathrm{MHz}, \mathrm{ppm}\right): \delta=10.79(\mathrm{~s}, 1 \mathrm{H}$, iminium- $H), 7.54(\mathrm{t}, J=7.5 \mathrm{~Hz}, 1 \mathrm{H}$, $p-\mathrm{H}), 7.35(\mathrm{t}, J=8.0 \mathrm{~Hz}, 2 \mathrm{H}, m-\mathrm{H}), 4.59\left(\mathrm{~d}, J=10.5 \mathrm{~Hz}, 1 \mathrm{H}, \mathrm{C} H_{2}\right), 3.45(\mathrm{~d}, J=10.5 \mathrm{~Hz}, 1 \mathrm{H}$, $\mathrm{CH}_{2}$ ), 3.06 (sept, $J=7.0 \mathrm{~Hz}, 1 \mathrm{H}, \mathrm{C} H \mathrm{Me}_{2}$ ), 2.78 (d, $J=14.0 \mathrm{~Hz}, 1 \mathrm{H}, \mathrm{CH}_{2}$ ), 2.61 (sept, $J=7.0$ $\mathrm{Hz}, 1 \mathrm{H}, \mathrm{C} H \mathrm{Me}_{2}$ ), 2.45 (d, J=14.0 Hz, 1H, CH ( $_{2}, 2.06$ (s, 3H, CMe), 1.62 (s, 3H, CMe), 1.60 (s, 3H, CMe), 1.35 (d, J=7.0 Hz, 3H, CHMe $), 1.34$ (d, $J=7.0 \mathrm{~Hz}, 3 \mathrm{H}, \mathrm{CHMe}$ ), 1.32 (d, $J=$ $7.0 \mathrm{~Hz}, 3 \mathrm{H}, \mathrm{CHMe}), 1.20(\mathrm{~d}, J=7.0 \mathrm{~Hz}, 3 \mathrm{H}, \mathrm{CHMe}) .{ }^{13} \mathrm{C}\left\{{ }^{1} \mathrm{H}\right\} \mathrm{NMR}\left(\mathrm{CDCl}_{3}, 125 \mathrm{MHz}\right.$, ppm): $\delta=189.8$ (iminium $C$ ), 144.9, 144.6, 132.3, 128.6, 125.7, $125.6(\operatorname{ArC}), 83.0\left(\mathrm{C}_{\mathrm{q}}\right), 54.0$ $\left(C_{\mathrm{q}}\right), 47.9\left(\mathrm{CH}_{2}\right), 30.3,29.9,28.4,27.9,27.8,27.5,27.4,22.4,21.8,12.4\left(\mathrm{CH}_{2} \mathrm{I}\right)$. HRMS: HRMS: $\mathrm{m} / \mathrm{z}$ calculated for $\left[\mathrm{C}_{20} \mathrm{H}_{31} \mathrm{IN}\right]^{+} 412.1496$, found 412.1497 . 


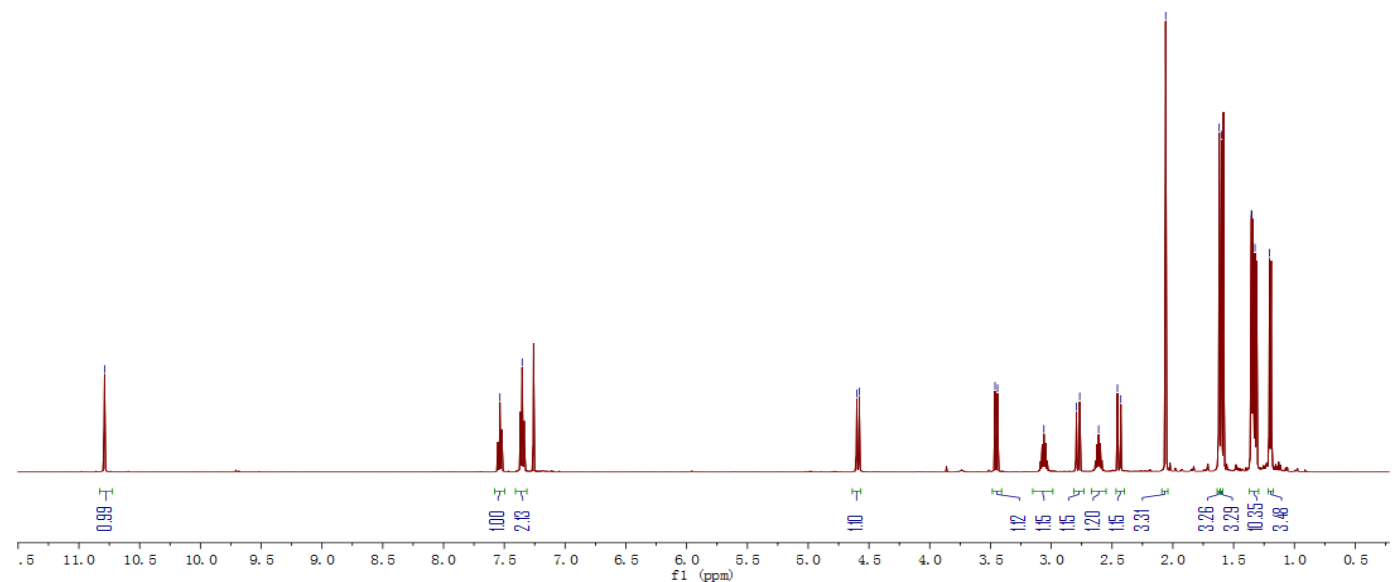

Figure S17. ${ }^{1} \mathrm{H}$ NMR of $\mathbf{4 f}\left(\mathrm{CDCl}_{3}, 500 \mathrm{MHz}\right)$.

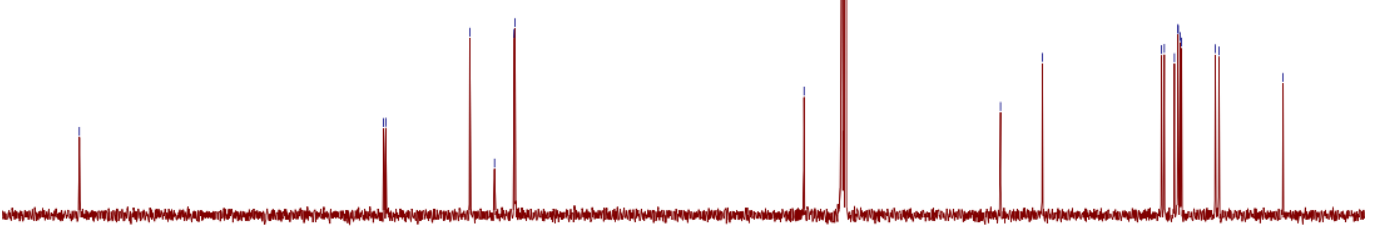

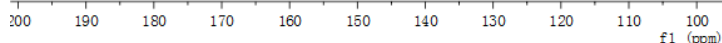

Figure S18. ${ }^{13} \mathrm{C}\left\{{ }^{1} \mathrm{H}\right\}$ NMR of $\mathbf{4 f}\left(\mathrm{CDCl}_{3}, 125 \mathrm{MHz}\right)$.

\section{Cyclic iminium salt $\mathbf{4 g}$}

THF $(20 \mathrm{~mL})$ was added to a mixture of cyclic iminium salt $\mathbf{4 f}$ (4.31 g, $8 \mathrm{mmol}), \mathrm{Ph}_{2} \mathrm{PH}(1.64 \mathrm{~g}, 8.8 \mathrm{mmol})$ and ${ }^{i} \mathrm{Pr}_{2} \mathrm{NEt}$ (3.98 g, $32 \mathrm{mmol})$. The reaction mixture was heated at $100{ }^{\circ} \mathrm{C}$ for 2 days. After THF was removed in vacuo, $\mathrm{K}_{3} \mathrm{PO}_{4}(2.04 \mathrm{~g}, 9.6 \mathrm{mmol})$ and

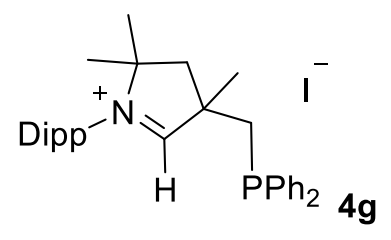
$\mathrm{CH}_{3} \mathrm{CN}(20 \mathrm{~mL})$ were added to the solid and the mixture was stirred at room temperature for 
12 hours. After $\mathrm{CH}_{3} \mathrm{CN}$ was removed in vacuo, the solid was extracted with DCM $(75 \mathrm{~mL})$. After DCM was removed in vacuo, the solid was washed with THF $(100 \mathrm{~mL})$ to afford a pale yellow powder (3.3 g, 69\%). m.p. $151^{\circ} \mathrm{C}$.

${ }^{1} \mathrm{H}$ NMR $\left(\mathrm{CDCl}_{3}, 300 \mathrm{MHz}, \mathrm{ppm}\right): \delta=11.01(\mathrm{~s}, 1 \mathrm{H}$, iminium- $H)$, 7.75-7.65 (m, 3H, $\left.\mathrm{Ar} H\right)$, 7.60-7.45 (m, 5H, ArH), 7.40-7.25 (m, 5H, $\mathrm{ArH}$ ), 3.25-3.10 (m, 3H, $\mathrm{CHMe}$ and $\mathrm{Ph}_{2} \mathrm{PCH}_{2}$ ), 2.68 (sept, $J=6.6 \mathrm{~Hz}, 1 \mathrm{H}, \mathrm{C} H \mathrm{Me}_{2}$ ), 2.36 (d, $\left.J=14.1 \mathrm{~Hz}, 1 \mathrm{H}, \mathrm{CH}{ }_{2}\right), 2.18$ (d, $J=14.1 \mathrm{~Hz}, 1 \mathrm{H}$, $\mathrm{CH}_{2}$ ), 1.97 (s, 3H, CMe), 1.52 (s, 3H, CMe), 1.40-1.30 (m, 12H, CHMe $), 1.26$ (d, J=6.6 Hz, $3 \mathrm{H}, \mathrm{CHMe} 2) .{ }^{13} \mathrm{C}\left\{{ }^{1} \mathrm{H}\right\} \mathrm{NMR}\left(\mathrm{CDCl}_{3}, 125 \mathrm{MHz}, \mathrm{ppm}\right): \delta=192.0(\mathrm{~d}, J=5.3 \mathrm{~Hz}$, iminium $C)$, 145.3, 144.8, 137.3 (d, $J=7.5 \mathrm{~Hz}), 136.1,134.2,134.0,132.8,132.6,132.0,130.2,129.4$, 129.3, 128.9, 128.8, 125.6, $125.5(\operatorname{ArC}), 82.4\left(\mathrm{C}_{\mathrm{q}}\right), 53.1(\mathrm{~d}, J=7.5 \mathrm{~Hz}), 47.0(\mathrm{~d}, J=6.9 \mathrm{~Hz})$, $37.6(\mathrm{~d}, J=15.2 \mathrm{~Hz}), 30.2(\mathrm{~d}, J=6.9 \mathrm{~Hz}), 30.0,29.9,28.8,28.5,27.8,27.7,22.4,22.0 .{ }^{31} \mathrm{P}$ $\left\{{ }^{1} \mathrm{H}\right\} \mathrm{NMR}\left(\mathrm{CDCl}_{3}, 121 \mathrm{MHz}, \mathrm{ppm}\right):-19.0$. HRMS: $\mathrm{m} / \mathrm{z}$ calculated for $\left[\mathrm{C}_{32} \mathrm{H}_{41} \mathrm{NP}\right]^{+}$ 470.2971, found 470.2972 .

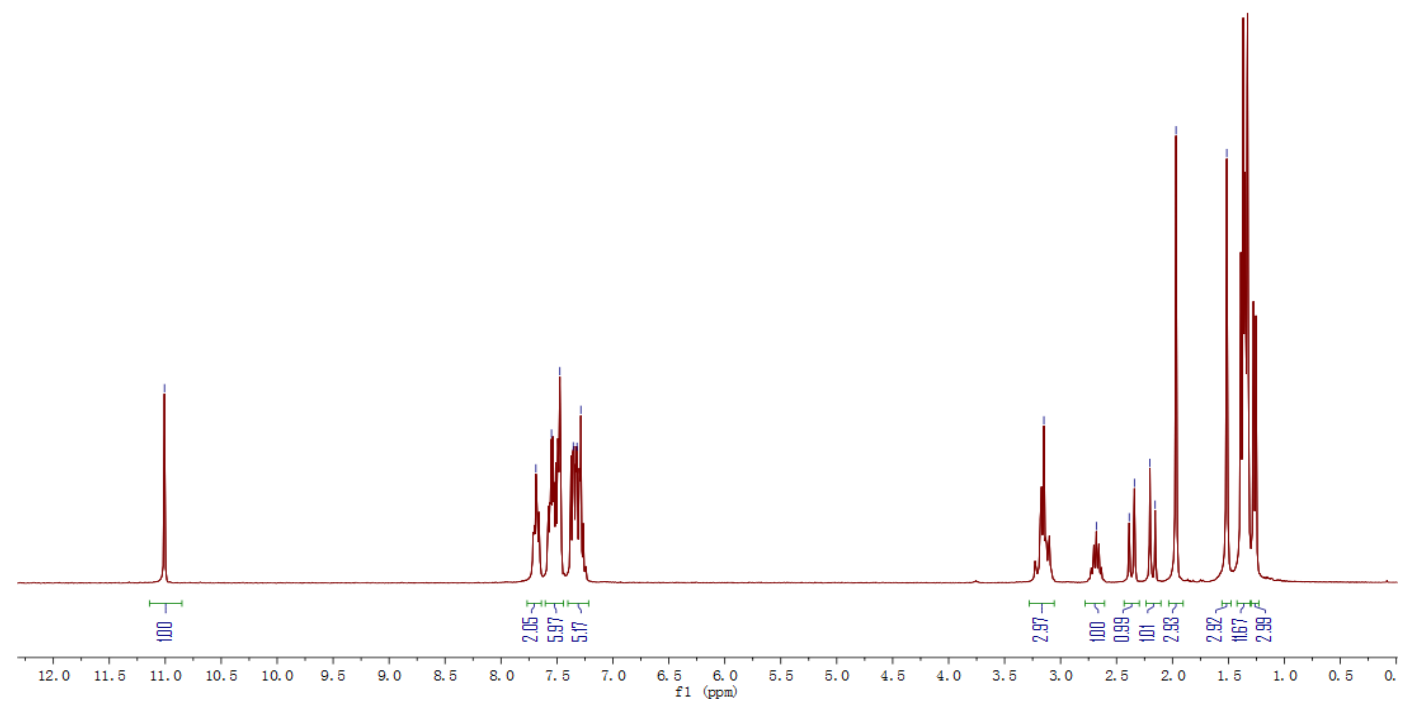

Figure S19. ${ }^{1} \mathrm{H}$ NMR of $\mathbf{4 g}\left(\mathrm{CDCl}_{3}, 300 \mathrm{MHz}\right)$. 


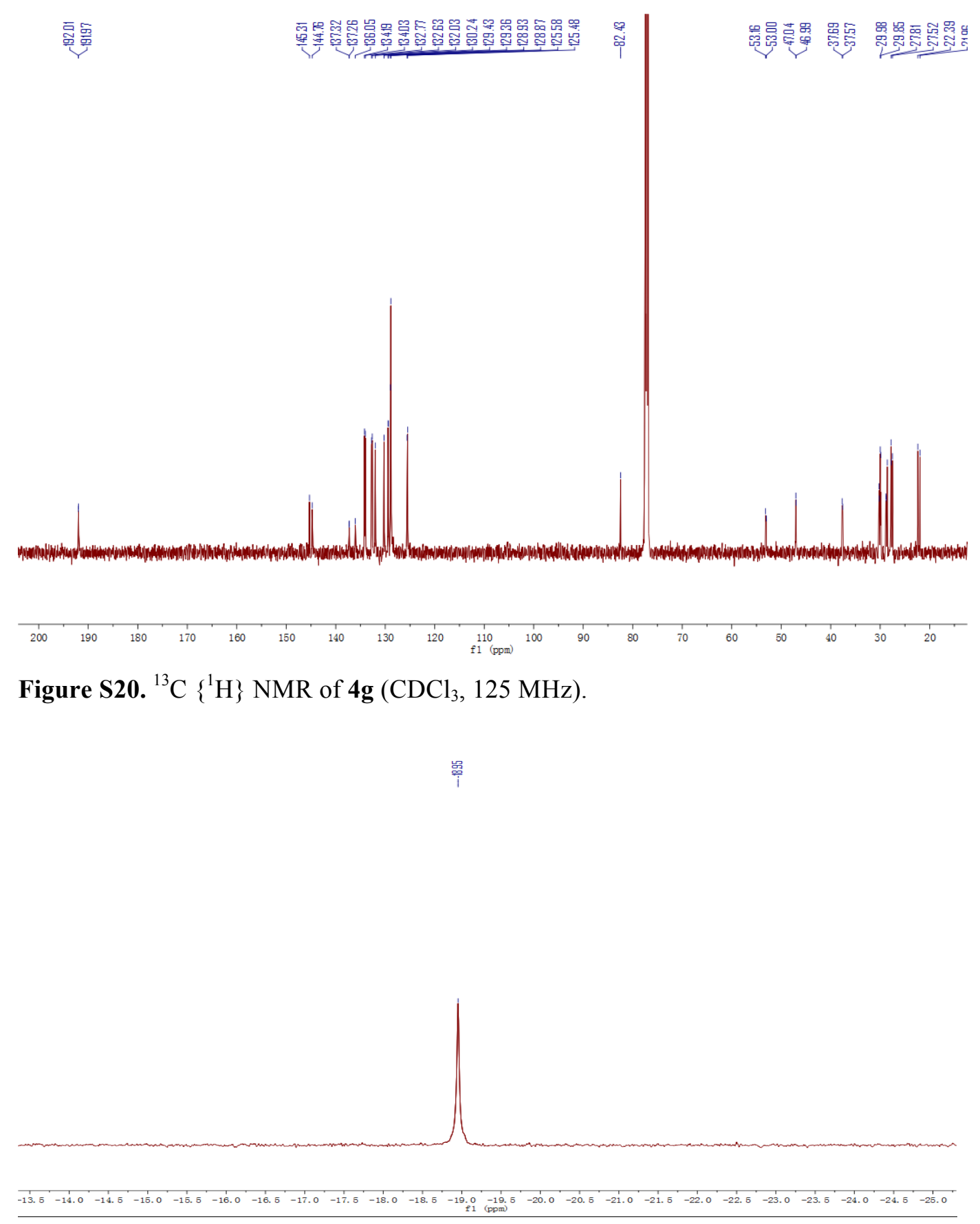

Figure S21. ${ }^{31} \mathrm{P}\left\{{ }^{1} \mathrm{H}\right\} \mathrm{NMR}$ of $\mathbf{4 g}\left(\mathrm{CDCl}_{3}, 121 \mathrm{MHz}\right)$.

\section{Cyclic iminium $\mathbf{5 H}^{+} \mathbf{B F}{ }^{-}$}

A solution of $\mathrm{HCl}$ in diethylether $(200 \mathrm{~mL}, 2.0 \mathrm{M}, 400 \mathrm{mmol})$ was added to a solution of crude alkenyl aldimine $2(200 \mathrm{mmol}$, from the last step) in diethylether $(100 \mathrm{~mL})$ at $-78{ }^{\circ} \mathrm{C}$. Precipitation of a colorless powder was immediately observed.

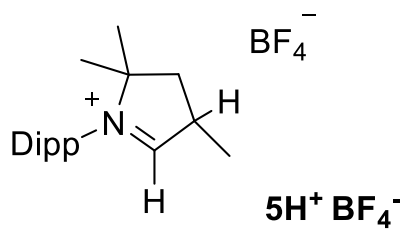

After 15 minutes the mixture was warmed to room temperature and stirring was continued for an additional 15 minutes. The solution was heated in a pressure tube at $110^{\circ} \mathrm{C}$ for $24 \mathrm{~h}$ and then cooled to room temperature. The diethylether solution was removed via syringe and the 
solid was washed with ether until the solution was colorless. The solid was dried under vacuum to afford a colorless powder, which was the iminium salt with $\mathrm{HCl}_{2}{ }^{-}$anion. To a Schlenk tube containing the iminium salt $\left(\mathrm{X}=\mathrm{HCl}_{2}{ }^{-}\right)$and $\mathrm{NaBF}_{4}(43.91 \mathrm{~g}, 400 \mathrm{mmol})$ was added $\mathrm{H}_{2} \mathrm{O}(200 \mathrm{~mL})$. The mixture was stirred for $1 \mathrm{~h}$ at room temperature and filtered. The solid was washed with $\mathrm{H}_{2} \mathrm{O}(100 \mathrm{~mL}, 2 \mathrm{x})$, diethylether $(100 \mathrm{~mL}, 2 \mathrm{x})$, pentane $(100 \mathrm{~mL}, 2 \mathrm{x})$, and then dried under vacuum to get a colorless powder $(39.00$ g, $54 \%)$. m.p. $232-233{ }^{\circ} \mathrm{C}$ (dec.).

${ }^{1} \mathrm{H}$ NMR $\left(\mathrm{CDCl}_{3}, 300 \mathrm{MHz}, \mathrm{ppm}\right): \delta=8.81(\mathrm{~s}, 1 \mathrm{H}$, iminium- $H), 7.50(\mathrm{t}, J=10.4 \mathrm{~Hz}, 1 \mathrm{H}$, $p-\mathrm{H}), 7.32(\mathrm{t}, J=10.4 \mathrm{~Hz}, 2 \mathrm{H}, m-\mathrm{H}), 4.12\left(\mathrm{~m}, 1 \mathrm{H}, \mathrm{CHCH}_{3}\right), 2.86-2.70\left(\mathrm{~m}, 2 \mathrm{H}, \mathrm{CH}_{2}\right.$ and $\mathrm{C} H \mathrm{Me}_{2}$ ), 2.60 (sept, $\left.J=6.9 \mathrm{~Hz}, 1 \mathrm{H}, \mathrm{C} H \mathrm{Me}_{2}\right), 2.11\left(\mathrm{dd}, J=17.6 \mathrm{~Hz}, J=11.6 \mathrm{~Hz}, 1 \mathrm{H}, \mathrm{CH}_{2}\right.$ ), 1.57 (s, 3H, CMe), 1.57 (d, J=9.6 Hz, 1H, CHMe), 1.43 (s, 3H, CMe), 1.36 (d, J=6.8 Hz, $3 \mathrm{H}, \mathrm{CHMe}$ ), 1.29 (d, J=6.8 Hz, 3H, CHMe $), 1.15$ (d, J=6.8 Hz, 3H, CHMe $), 1.08$ (d, J= $6.8 \mathrm{~Hz}, 3 \mathrm{H}, \mathrm{CHMe} 2) .{ }^{13} \mathrm{C}\left\{{ }^{1} \mathrm{H}\right\} \mathrm{NMR}\left(\mathrm{CDCl}_{3}, 125 \mathrm{MHz}, \mathrm{ppm}\right): \delta=190.5$ (iminium $\mathrm{C}$ ), 144.9, 144.5, 131.9, 129.4, 126.0, 125.0 ( $\mathrm{ArC}), 83.8\left(\mathrm{C}_{\mathrm{q}}\right), 43.7\left(\mathrm{CH}_{2}\right), 41.8(\mathrm{CH}), 29.9,29.3,26.5$, 26.4, 26.2, 23.0, 21.9, 16.3. HRMS: $\mathrm{m} / \mathrm{z}$ calculated for $\left[\mathrm{C}_{19} \mathrm{H}_{30} \mathrm{~N}\right]^{+} 272.2373$, found 272.2376.

$\bar{\Phi}$

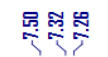

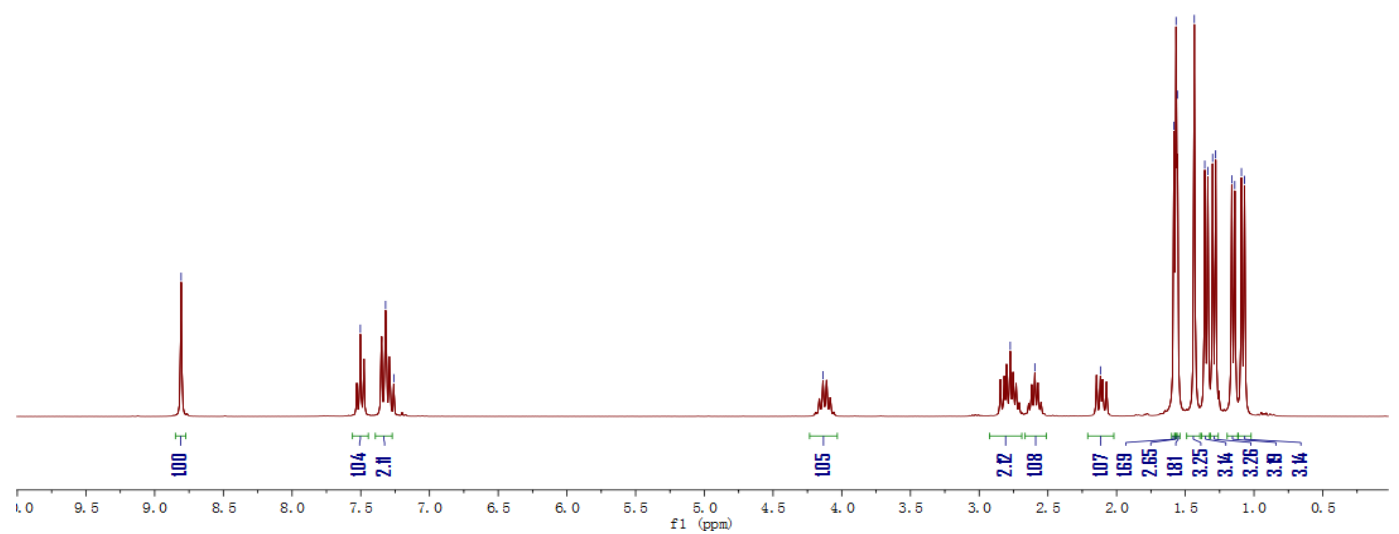

Figure S22. ${ }^{1} \mathrm{H} \mathrm{NMR}$ of $\mathbf{5 H}^{+} \mathbf{B F}_{4}^{-}\left(\mathrm{CDCl}_{3}, 300 \mathrm{MHz}\right)$. 

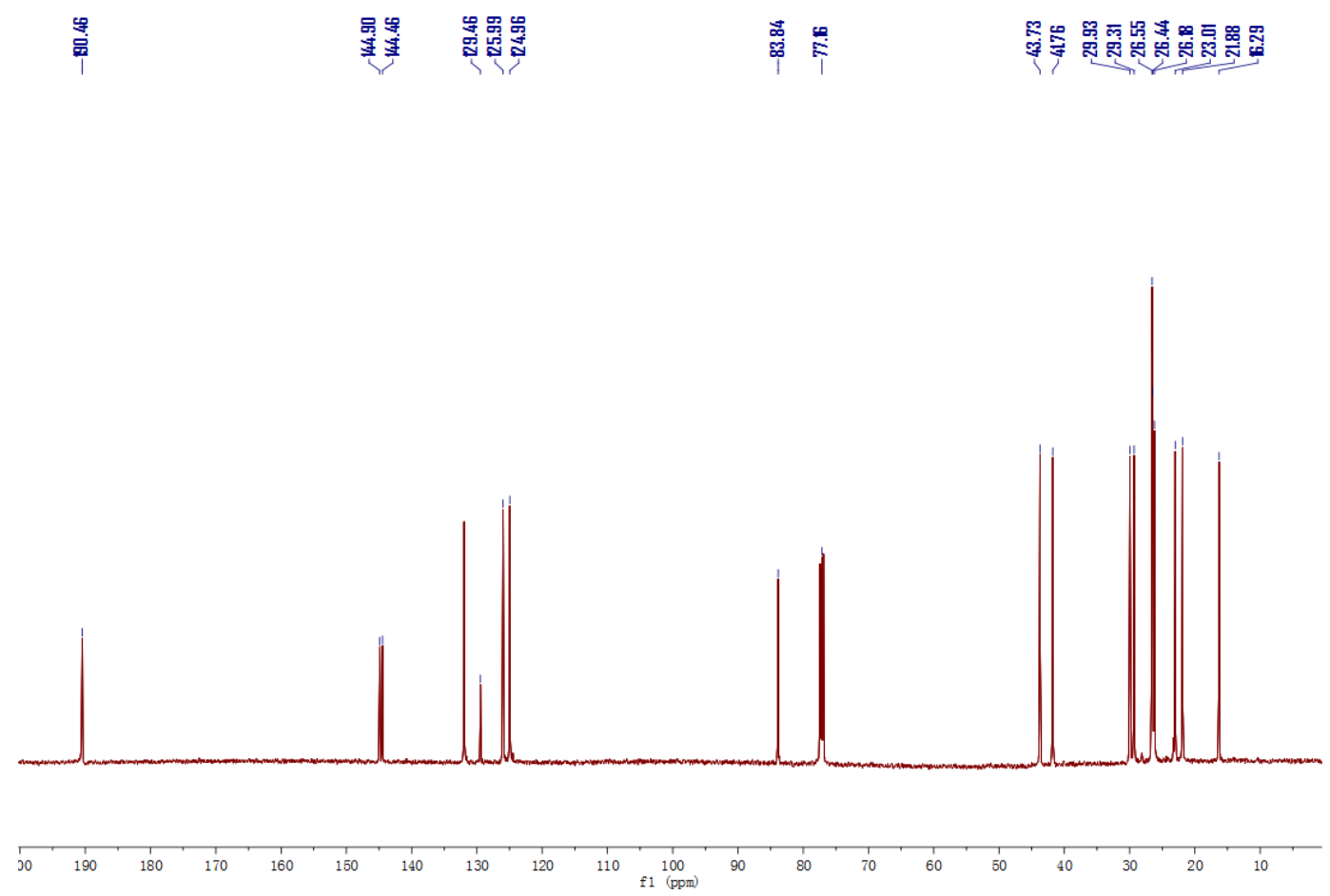

Figure S23. ${ }^{13} \mathrm{C}\left\{{ }^{1} \mathrm{H}\right\} \mathrm{NMR}$ of $\mathbf{5 H}^{+} \mathbf{B F}_{4}^{-}\left(\mathrm{CDCl}_{3}, 125 \mathrm{MHz}\right)$.

\section{1-(2,6-diisopropylphenyl)-2,2,4-trimethyl-2,3-dihydro-1H-pyrrole 5}

Ammonium hydroxide solution $(28-30 \%, 40 \mathrm{~mL})$ was added to an ether solution $(100 \mathrm{~mL})$ of iminium salt $\mathbf{5 H}^{+} \mathbf{B F}_{\mathbf{4}}^{-}(39.00 \mathrm{~g}, 108 \mathrm{mmol})$. The biphasic mixture was stirred at room temperature for $30 \mathrm{~min}$. The organic phase was separated and the aqueous phase was further extracted with ether $(3 \times 100 \mathrm{~mL})$. The ether solution was combined and<smiles>CC1=C(C)N([PbH])C(C)(C)C1</smiles>
dried with $\mathrm{MgSO}_{4}$ overnight. After evaporation of the solvent under vacuum, a pale yellow oil (27.8 g, 94\% based on enamine, $51 \%$ based on 2,6-diisopropylaniline). ${ }^{1} \mathrm{H}$ NMR ( $\mathrm{CDCl}_{3}$, $300 \mathrm{MHz}, \mathrm{ppm}): \delta=7.20-7.08(\mathrm{~m}, 3 \mathrm{H}, \mathrm{Ar} H), 5.57(\mathrm{~s}, 1 \mathrm{H},=\mathrm{CH}), 3.50$ (sept, $J=6.9 \mathrm{~Hz}, 2 \mathrm{H}$, $\mathrm{CHMe}$ ), 2.49 (s, 2H, $\left.\mathrm{CH}_{2}\right), 1.71(\mathrm{~s}, 3 \mathrm{H},=\mathrm{CMe}), 1.23(\mathrm{~d}, J=6.9 \mathrm{~Hz}, 6 \mathrm{H}, \mathrm{CHMe}), 1.16(\mathrm{~s}, 6 \mathrm{H}$, $\left.\mathrm{CMe}_{2}\right), 1.07\left(\mathrm{~d}, J=6.9 \mathrm{~Hz}, 6 \mathrm{H}, \mathrm{CHMe} e_{2}\right){ }^{13} \mathrm{C}\left\{{ }^{1} \mathrm{H}\right\} \mathrm{NMR}\left(\mathrm{CDCl}_{3}, 125 \mathrm{MHz}, \mathrm{ppm}\right): \delta=151.4$, 137.9, 134.1, 126.9, 123.8, 104.0 ( $\mathrm{ArC}$ and $C \mathrm{H}=C \mathrm{Me}), 65.0,51.1,28.5,28.1,26.4,23.5,14.0$. HRMS: $\mathrm{m} / \mathrm{z}$ calculated for $\left[\mathrm{C}_{19} \mathrm{H}_{30} \mathrm{~N}\right]^{+} 272.2373$, found 272.2376 . 

ํㅜำ문

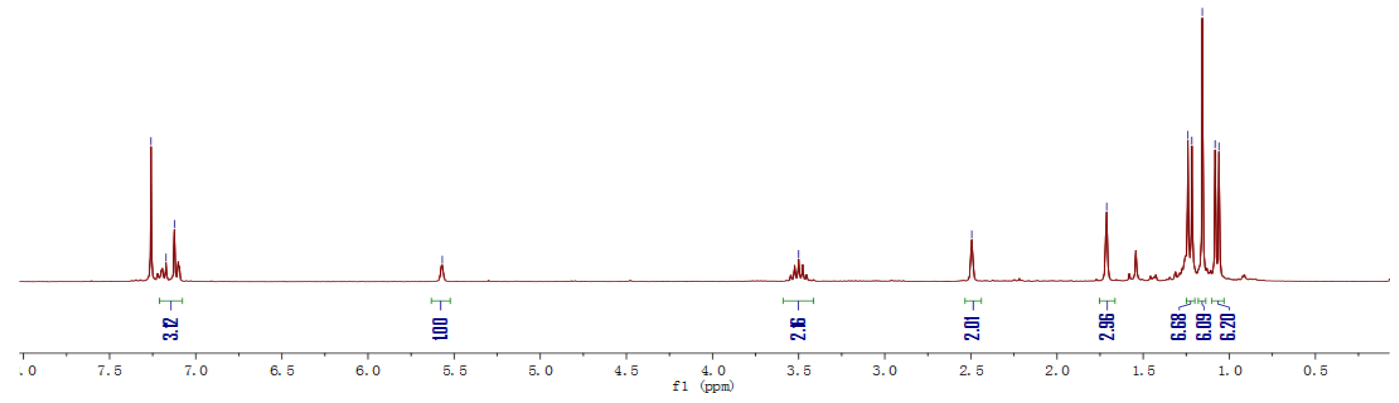

Figure S24. ${ }^{1} \mathrm{H}$ NMR of $\mathbf{5 H}^{+} \mathbf{B F}_{4}{ }^{-}\left(\mathrm{CDCl}_{3}, 300 \mathrm{MHz}\right)$.

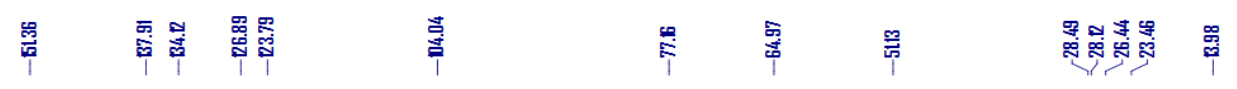

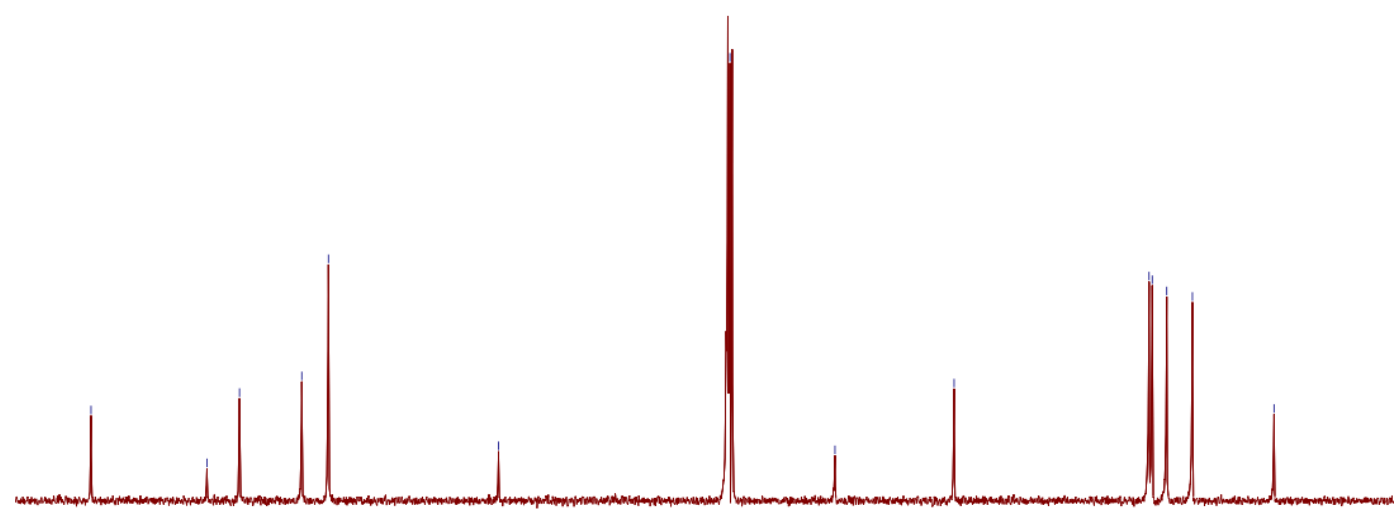

Figure S25. ${ }^{13} \mathrm{C}\left\{{ }^{1} \mathrm{H}\right\} \mathrm{NMR}$ of $\mathbf{5 H}^{+} \mathbf{B F}_{4}^{-}\left(\mathrm{CDCl}_{3}, 125 \mathrm{MHz}\right)$.

\section{Free Carbenes}

\section{Carbene 6a}

$\mathrm{Et}_{2} \mathrm{O}(10 \mathrm{~mL})$ was added to a mixture of cyclic iminium salt $4 \mathbf{a}$ $(350 \mathrm{mg}, 0.736 \mathrm{mmol})$ and KHMDS $(147 \mathrm{mg}, 2.0 \mathrm{mmol})$ under

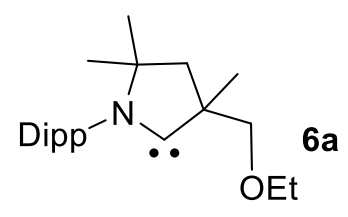


stirring at $-78^{\circ} \mathrm{C}$. The solvent was removed in vacuo after stirring for $15 \mathrm{~min}$ and the product was extracted with pentane. Evaporation of volatiles gave the carbene as a greasy, colorless solid in quantitative yield.

${ }^{1} \mathrm{H}$ NMR $\left(\mathrm{C}_{6} \mathrm{D}_{6}, 400 \mathrm{MHz}, \mathrm{ppm}\right): \delta=7.21(\mathrm{~m}, 3 \mathrm{H}, \operatorname{Ar} H), 4.05\left(\mathrm{~d}, J=10.6 \mathrm{~Hz}, 1 \mathrm{H}, \mathrm{CH}_{2}\right)$, $3.43(\mathrm{~m}, 1 \mathrm{H}), 3.42(\mathrm{~m}, 1 \mathrm{H}), 3.39\left(\mathrm{~d}, J=10.6 \mathrm{~Hz}, 1 \mathrm{H}, \mathrm{CH} \mathrm{C}_{2}\right), 3.31(\mathrm{~m}, 1 \mathrm{H}), 3.20(\mathrm{~m}, 1 \mathrm{H}), 2.15$ (d, $\left.J=12.5 \mathrm{~Hz}, 1 \mathrm{H}, \mathrm{CH}_{2}\right), 1.46\left(\mathrm{~d}, J=12.5 \mathrm{~Hz}, 1 \mathrm{H}, \mathrm{CH}_{2}\right), 1.33(\mathrm{~b}, 3 \mathrm{H}), 1.32(\mathrm{~b}, 3 \mathrm{H}), 1.30$ (b, $3 \mathrm{H}), 1.26(\mathrm{~b}, 3 \mathrm{H}), 1.24(\mathrm{~b}, 3 \mathrm{H}), 1.23(\mathrm{~b}, 6 \mathrm{H}), 1.15(\mathrm{~b}, 3 \mathrm{H}) .{ }^{13} \mathrm{C}\left\{{ }^{1} \mathrm{H}\right\}$ NMR $\left(\mathrm{C}_{6} \mathrm{D}_{6}, 125 \mathrm{MHz}\right.$, ppm): $\delta=311.0$ (carbene $C$ ), 146.8, 146.1, 138.3, 127.7, 124.1, $123.8(\operatorname{ArC}), 83.7\left(C_{\mathrm{q}}\right), 78.1$ $\left(\mathrm{CH}_{2}\right), 66.8\left(\mathrm{CH}_{2}\right), 63.7,45.8\left(\mathrm{CH}_{2}\right), 29.9,29.6,29.1,28.6,26.8,26.4,23.1,22.1,22.0,15.6$.

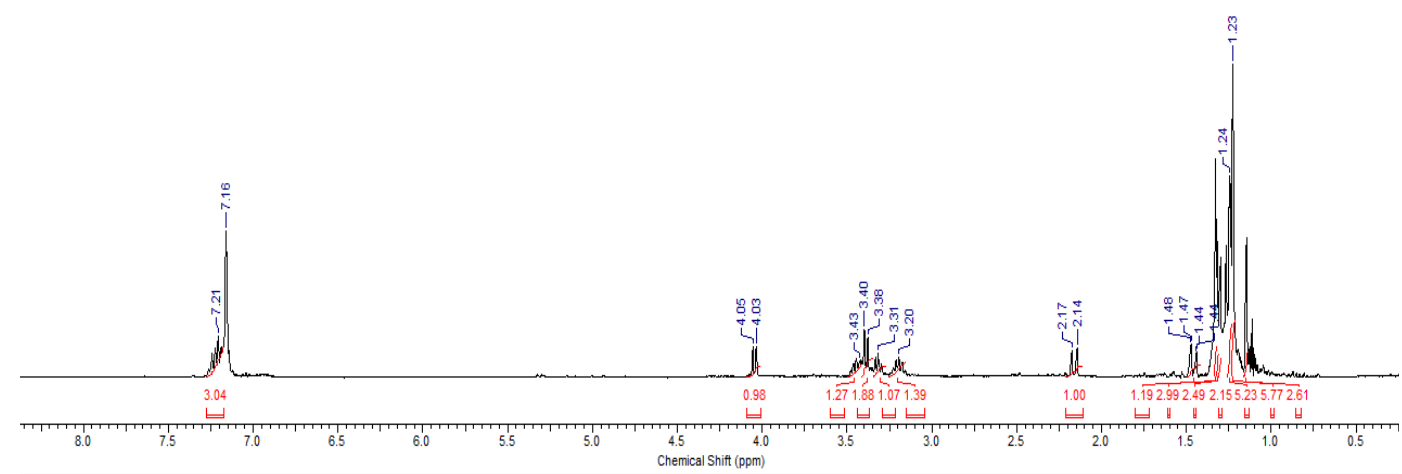

Figure S26. ${ }^{1} \mathrm{H}$ NMR of $6 \mathbf{a}\left(\mathrm{C}_{6} \mathrm{D}_{6}, 400 \mathrm{MHz}\right)$.

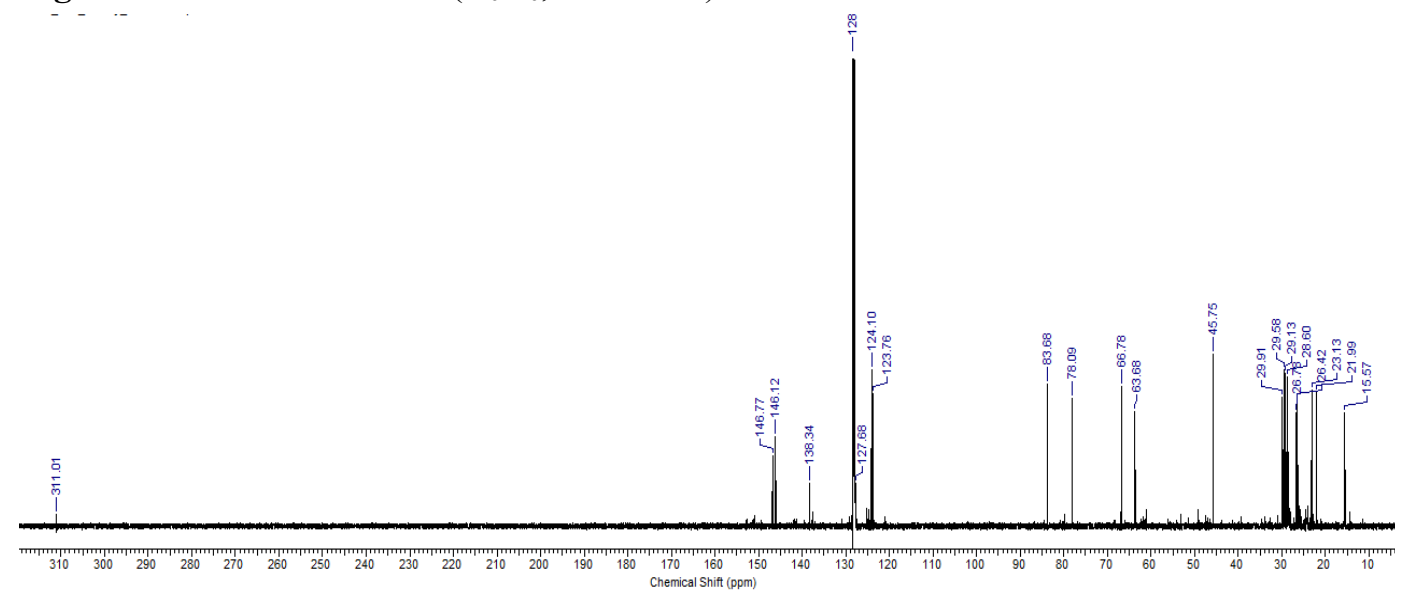

Figure S27. ${ }^{13} \mathrm{C}\left\{{ }^{1} \mathrm{H}\right\} \mathrm{NMR}$ of $\mathbf{6 a}\left(\mathrm{C}_{6} \mathrm{D}_{6}, 125 \mathrm{MHz}\right)$.

\section{Carbene 6b}

$\mathrm{C}_{6} \mathrm{D}_{6}$ was added to a mixture of cyclic iminium salt $\mathbf{4 b}(20.7 \mathrm{mg}$, $0.05 \mathrm{mmol}$ ) and KHMDS (10.0 mg, $0.05 \mathrm{mmol})$. It was agitated for $1 \mathrm{~min}$ and the NMR was taken after $20 \mathrm{~min}$.

${ }^{1} \mathrm{H}$ NMR $\left(\mathrm{C}_{6} \mathrm{D}_{6}, 300 \mathrm{MHz}, \mathrm{ppm}\right): \delta=7.18$ (br m, 3H), 5.01 (s, $1 \mathrm{H}$,

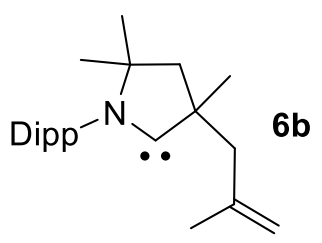
$\left.=\mathrm{CH}_{2}\right), 4.92\left(\mathrm{~s}, 1 \mathrm{H},=\mathrm{CH}_{2}\right), 3.12(\mathrm{~m}, 2 \mathrm{H}), 2.61(\mathrm{br}, 2 \mathrm{H}), 1.99(\mathrm{~s}, 3 \mathrm{H}), 1.75(\mathrm{~d}, J=12.9 \mathrm{~Hz}, 2 \mathrm{H}$, $\mathrm{CH}_{2}$ ), 1.47 (d, $\mathrm{J}=12.9 \mathrm{~Hz}, 2 \mathrm{H}, \mathrm{CH}_{2}$ ), 1.37 (s, 3H, CMe), 1.22 (br, $\left.12 \mathrm{H}\right), 1.08$ (br, 6H). ${ }^{13} \mathrm{C}$ $\left\{{ }^{1} \mathrm{H}\right\}$ NMR $\left(\mathrm{C}_{6} \mathrm{D}_{6}, 125 \mathrm{MHz}, \mathrm{ppm}\right): \delta=312.9$ (carbene $C$ ), 146.4, 146.1, 145.1, 137.9, 124.0, 123.9, 114.1, $114.0(\operatorname{ArC}), 81.8\left(\mathrm{C}_{\mathrm{q}}\right), 62.2,49.9,48.2,29.5,29.4,29.3,28.9,26.7,26.3,25.9$, 25.5, 22.1, 21.9. 


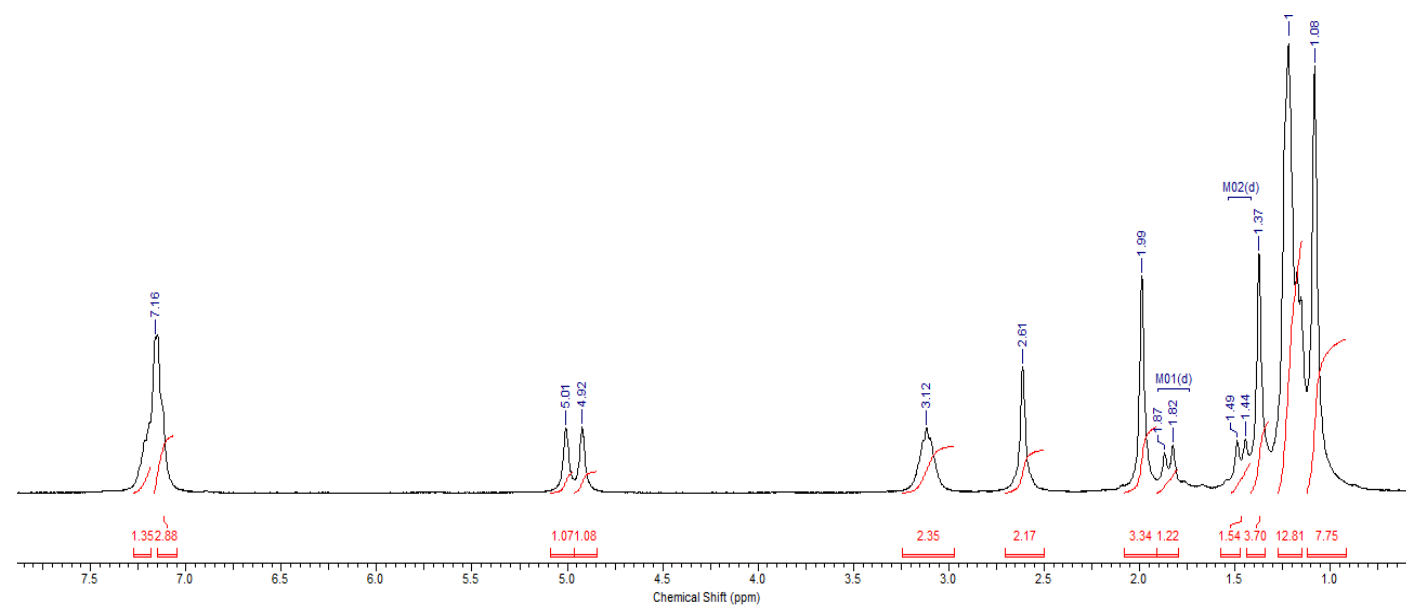

Figure S28. ${ }^{1} \mathrm{H}$ NMR of $\mathbf{6 b}\left(\mathrm{C}_{6} \mathrm{D}_{6}, 300 \mathrm{MHz}\right)$.

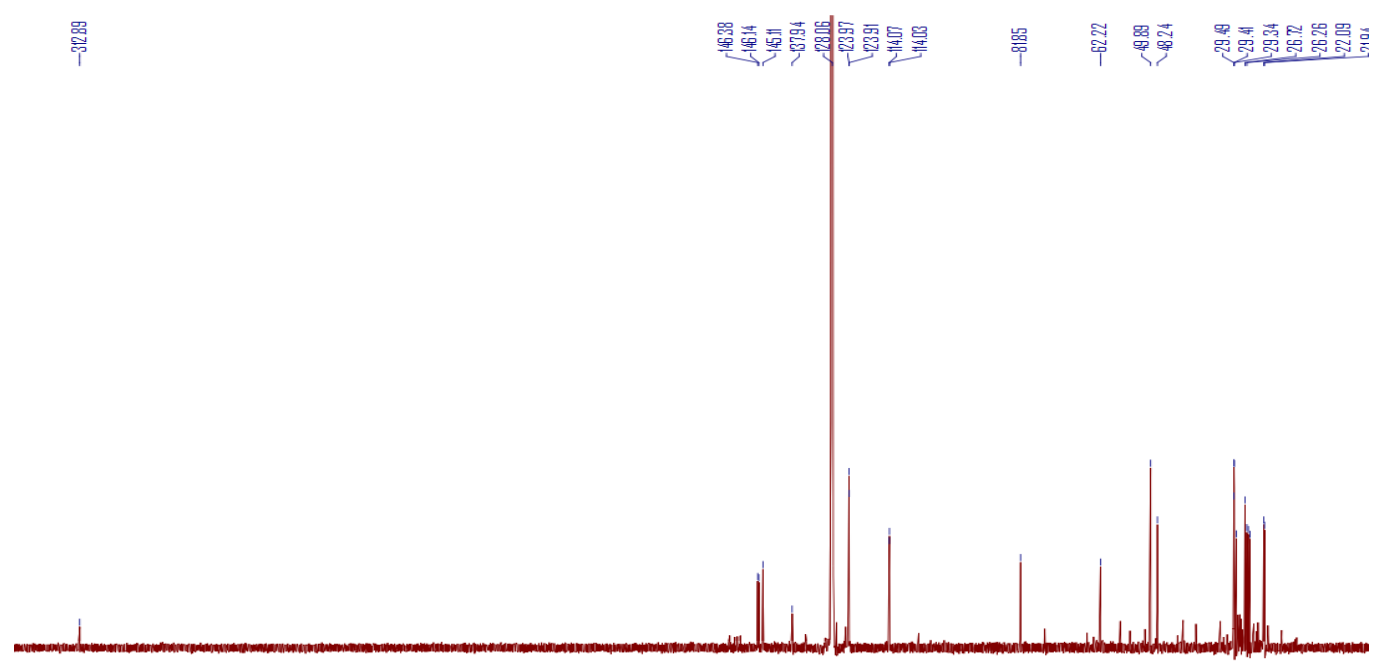

Figure S29. ${ }^{13} \mathrm{C}\left\{{ }^{1} \mathrm{H}\right\}$ NMR of $\mathbf{6 b}\left(\mathrm{C}_{6} \mathrm{D}_{6}, 125 \mathrm{MHz}\right)$.

\section{Carbene 6c}

$\mathrm{C}_{6} \mathrm{D}_{6}$ was added to a mixture of cyclic iminium salt $\mathbf{4 c}(20 \mathrm{mg}, 0.05$ mmol) and KHMDS (10.5 mg, $0.052 \mathrm{mmol})$. It was agitated for $1 \mathrm{~min}$ and the NMR was taken after $10 \mathrm{~min}$.

${ }^{1} \mathrm{H}$ NMR $\left(\mathrm{C}_{6} \mathrm{D}_{6}, 500 \mathrm{MHz}, \mathrm{ppm}\right): \delta=7.21(\mathrm{t}, J=7.5 \mathrm{~Hz}, 1 \mathrm{H}, p-\mathrm{H})$,

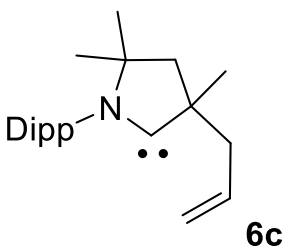
$7.14(\mathrm{~m}, 2 \mathrm{H}, m-\mathrm{H}), 6.06\left(\mathrm{~m}, 1 \mathrm{H}, \mathrm{CH}_{2}=\mathrm{CHCH}_{2}\right), 5.13-5.10\left(\mathrm{br} \mathrm{m}, 2 \mathrm{H},=\mathrm{CH}_{2}\right), 3.20-3.10(\mathrm{br} \mathrm{m}$, 2H, $\left.\mathrm{C} H \mathrm{Me}_{2}\right), 2.78\left(\mathrm{~d}, J=12.9 \mathrm{~Hz}, 6.0 \mathrm{~Hz}, 1 \mathrm{H}, \mathrm{CH}_{2}\right), 2.49$ (dd, $J=12.9 \mathrm{~Hz}, 8.8 \mathrm{~Hz}, 1 \mathrm{H}, \mathrm{CH}_{2}$ ), $1.74\left(\mathrm{~d}, J=12.5 \mathrm{~Hz}, 1 \mathrm{H}, \mathrm{CH}_{2}\right), 1.42\left(\mathrm{~d}, J=12.5 \mathrm{~Hz}, 1 \mathrm{H}, \mathrm{CH}_{2}\right) .1 .37$ (s, 3H, CMe), 1.23 (d, $J$ $=6.5 \mathrm{~Hz}, 6 \mathrm{H}, \mathrm{CHMe}$ ), 1.21 (d, $J=6.5 \mathrm{~Hz}, 3 \mathrm{H}, \mathrm{CHMe}$ ), 1.17 (d, J=6.5 Hz, 3H, CHMe $)$, 1.10 (s, 3H, CMe), 1.07 (s, 3H, CMe). ${ }^{13} \mathrm{C}\left\{{ }^{1} \mathrm{H}\right\}$ NMR $\left(\mathrm{C}_{6} \mathrm{D}_{6}, 125 \mathrm{MHz}, \mathrm{ppm}\right): \delta=313.2$ (carbene $C$ ), 146.1, 138.0, 137.1, 128.2, 127.9, 123.9, 123.8, $117.1(\operatorname{ArC}), 82.4\left(\mathrm{C}_{\mathrm{q}}\right), 62.2$, $47.2,46.3,29.6,29.3,29.3,28.8,26.3$ (2 C), 25.8, 21.9, 21.8 . 


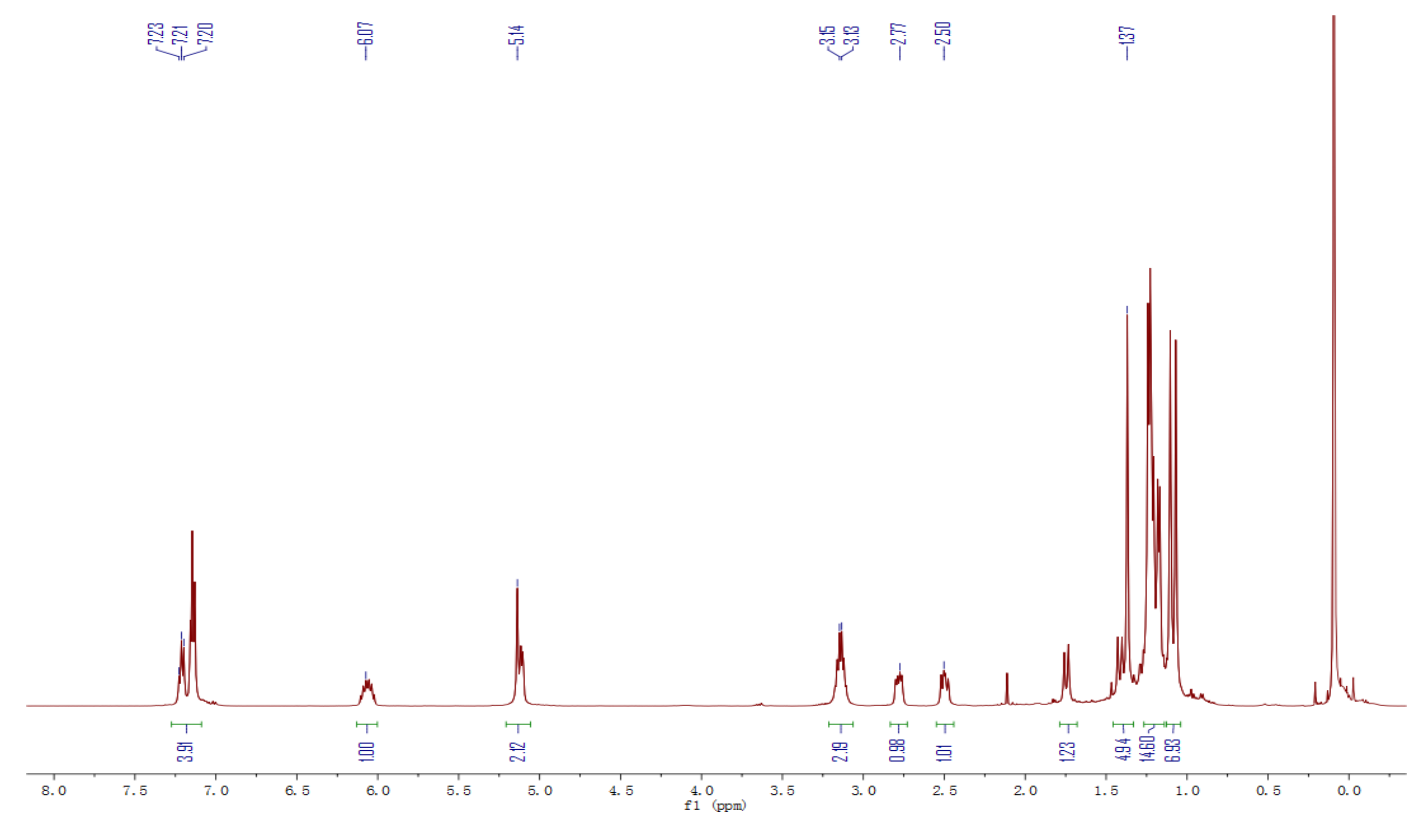

Figure S30. ${ }^{1} \mathrm{H}$ NMR of $6 \mathbf{c}\left(\mathrm{C}_{6} \mathrm{D}_{6}, 500 \mathrm{MHz}\right)$.

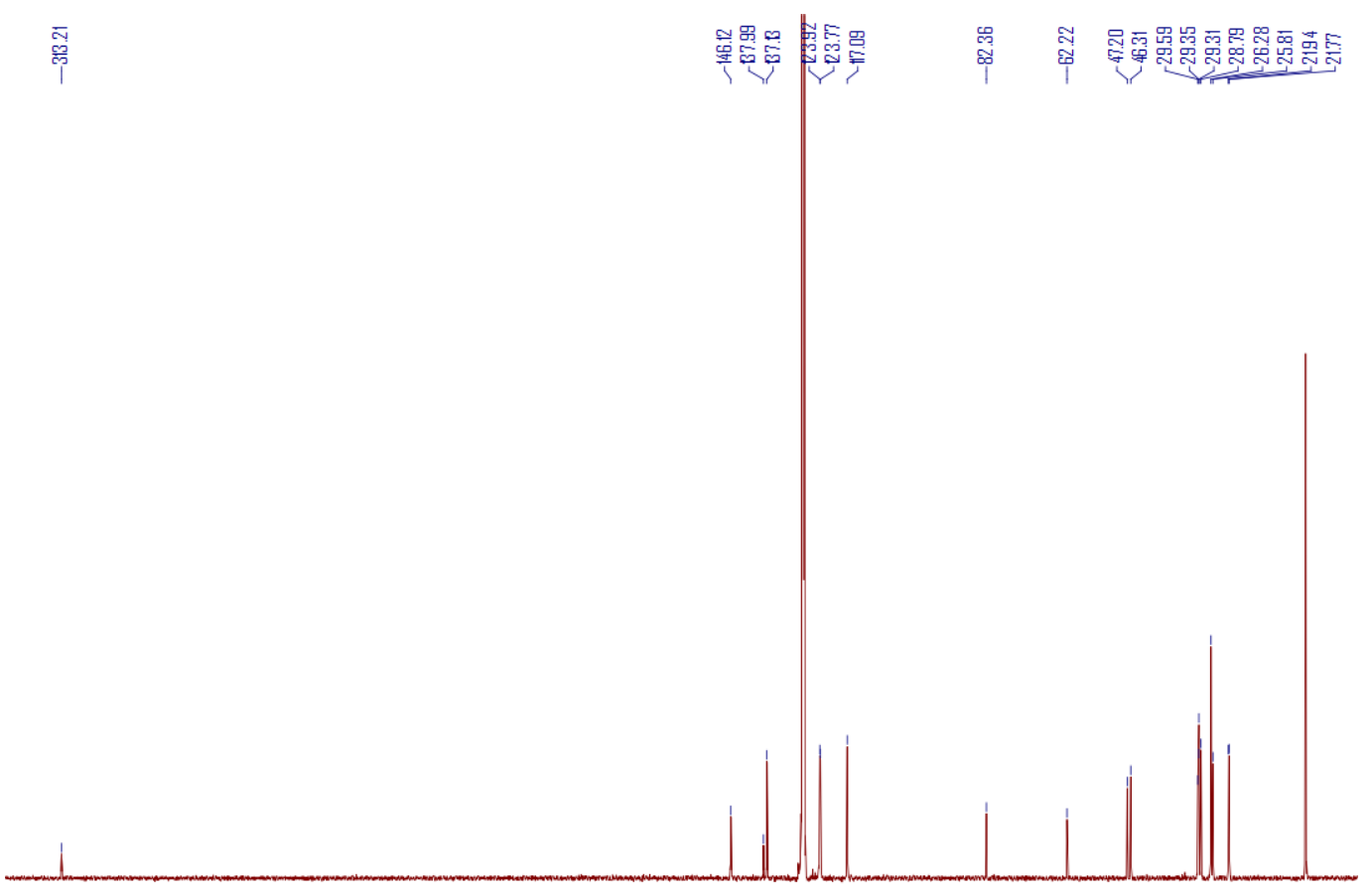

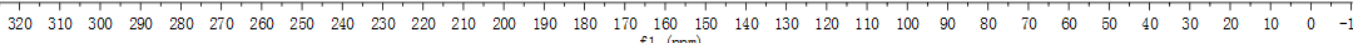

Figure S31. ${ }^{13} \mathrm{C}\left\{{ }^{1} \mathrm{H}\right\} \mathrm{NMR}$ of $\mathbf{6 c}\left(\mathrm{C}_{6} \mathrm{D}_{6}, 125 \mathrm{MHz}\right)$.

\section{Carbene 6d}

Deuterated benzene or ether was added to mixture of cyclic iminium salt 4d (20.8 mg, $0.05 \mathrm{mmol})$ and KHMDS (10.0 mg, $0.05 \mathrm{mmol}$ ). It was agitated for $1 \mathrm{~min}$ and the NMR was taken after $10 \mathrm{~min}$.

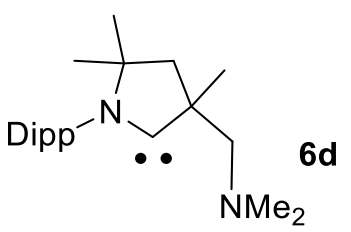


${ }^{1} \mathrm{H}$ NMR $\left(\mathrm{C}_{6} \mathrm{D}_{6}, 300 \mathrm{MHz}, \mathrm{ppm}\right): \delta=7.15(\mathrm{~m}, 3 \mathrm{H}, \mathrm{Ar} H), 3.24(\mathrm{~m}, 1 \mathrm{H}, \mathrm{CHMe}), 3.1(\mathrm{~m}, 1 \mathrm{H}$, $\left.\mathrm{C} H \mathrm{Me}_{2}\right), 2.94\left(\mathrm{~d}, J=13.4 \mathrm{~Hz}, 1 \mathrm{H}, \mathrm{CH}_{2}\right), 2.45\left(\mathrm{~d}, J=13.4 \mathrm{~Hz}, 1 \mathrm{H}, \mathrm{CH} \mathrm{H}_{2}\right), 2.34$ (s, 6H, NMe $)$, $2.13(\mathrm{~d}, J=12.5 \mathrm{~Hz}, 1 \mathrm{H}, \mathrm{CH}), 1.39(\mathrm{~d}, J=12.5 \mathrm{~Hz}, 1 \mathrm{H}, \mathrm{CH}), 1.30-1.08(\mathrm{br}, 21 \mathrm{H}) .{ }^{13} \mathrm{C}\left\{{ }^{1} \mathrm{H}\right\}$ NMR (diethylether, $125 \mathrm{MHz}, \mathrm{ppm}$ ): $\delta=312.6$ (carbene $C$ ), 146.5, 146.3, 138.1, 128.0, 123.8, 123.6 (ArC), 82.5( $\left.C_{\mathrm{q}}\right), 70.2,48.3,46.4,29.6,29.4,29.2,29.1,27.0,26.1,24.0,22.0,21.7$.

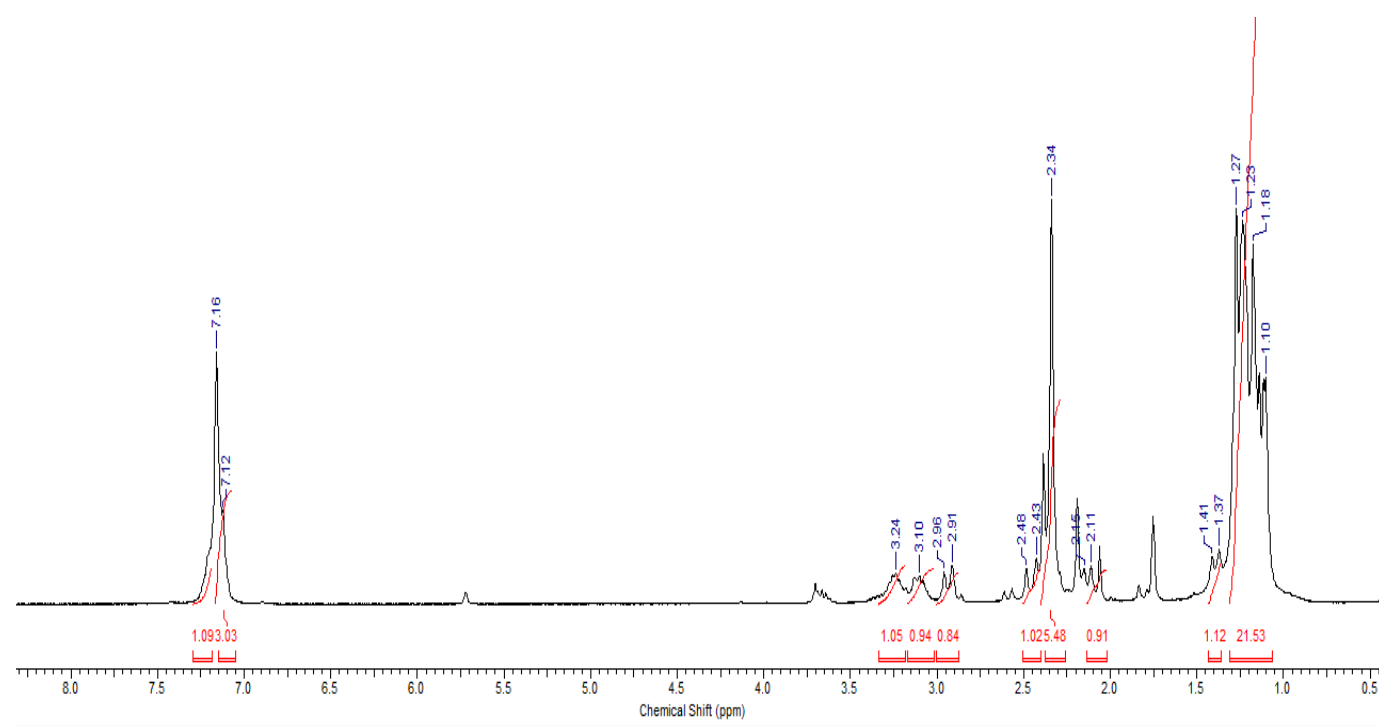

Figure S32. ${ }^{1} \mathrm{H}$ NMR of $\mathbf{6 d}\left(\mathrm{C}_{6} \mathrm{D}_{6}, 300 \mathrm{MHz}\right)$.

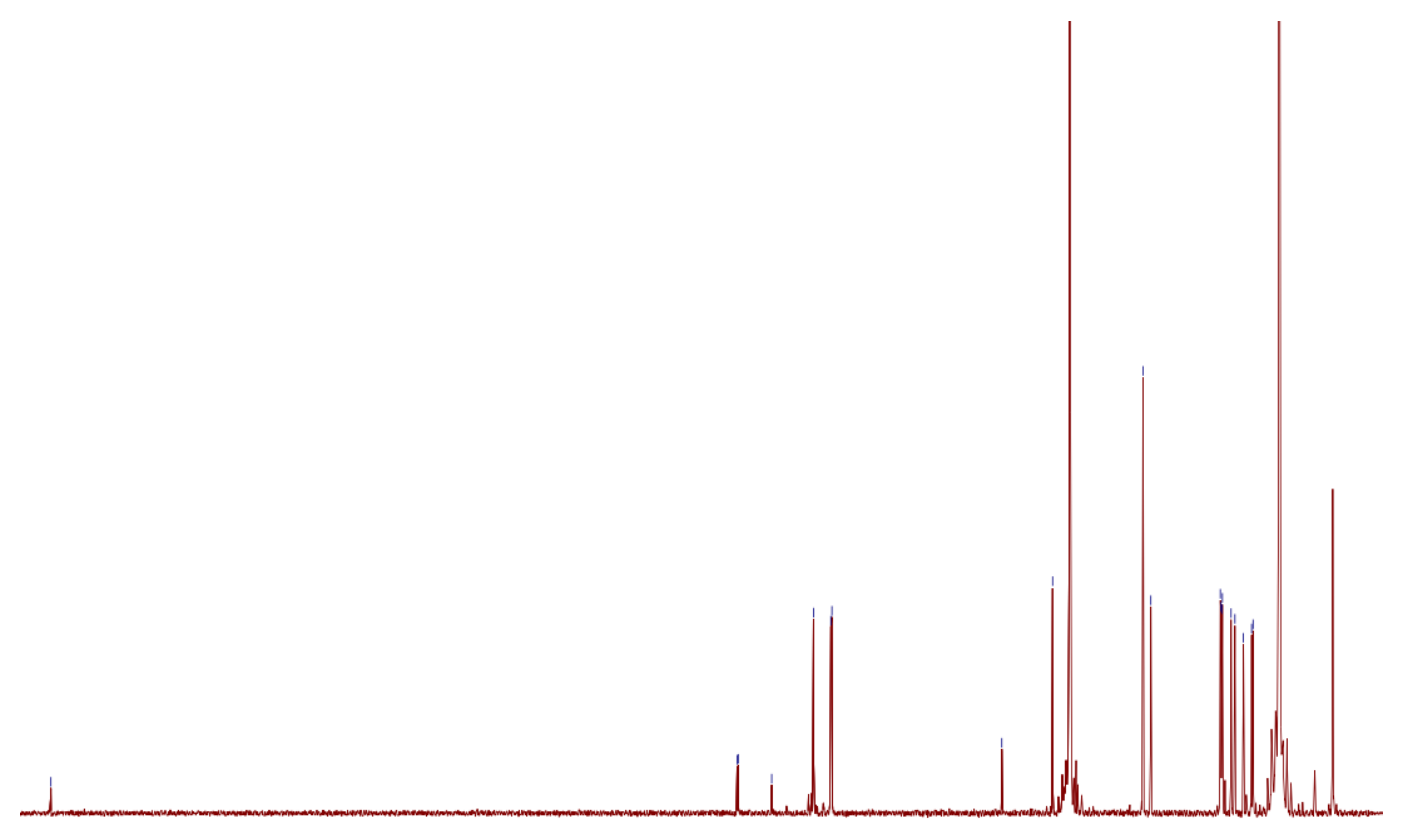

$\begin{array}{lllllllllllllllllllllllllllllllllllllll}310 & 300 & 290 & 280 & 270 & 260 & 250 & 240 & 230 & 220 & 210 & 200 & 190 & 180 & 170 & 160 & 150 & 140 & 130 & 120 & 110 & 100 & 90 & 80 & 70 & 60 & 50 & 40 & 30 & 20 & 10 & 0\end{array}$

Figure S33. ${ }^{13} \mathrm{C}\left\{{ }^{1} \mathrm{H}\right\}$ NMR of $\mathbf{6 d}$ (diethyl ether, $125 \mathrm{MHz}$ ). 


\section{Imino functionalized cyclic alkyl amino carbene $\mathbf{6 e}$}

$\mathrm{C}_{6} \mathrm{D}_{6}$ was added to a mixture of cyclic iminium salt $4 \mathrm{e}(30 \mathrm{mg}$, $0.05 \mathrm{mmol}$ ) and KHMDS (10 mg, $0.05 \mathrm{mmol})$. It was agitated for 1 min and the NMR was taken after $10 \mathrm{~min}$. This carbene decomposed slowly over the course of approximately $15 \mathrm{~h}$.

${ }^{1} \mathrm{H}$ NMR $\left(\mathrm{C}_{6} \mathrm{D}_{6}, 500 \mathrm{MHz}, \mathrm{ppm}\right)$ : 7.29-7.01 (m, 6H, $\left.\mathrm{Ar} H\right)$,

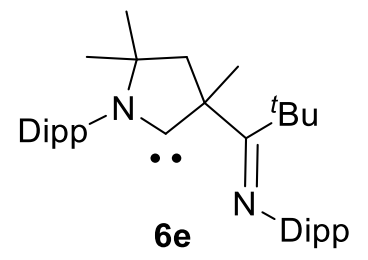
3.24-3.06 (m, 3H, CHMe $), 2.98$ (sept, $J=7.0 \mathrm{~Hz}, 1 \mathrm{H}, \mathrm{C} H \mathrm{Me}_{2}$ ), 2.39 (d, $J=13.0 \mathrm{~Hz}, 1 \mathrm{H}$, $\mathrm{CH}_{2}$ ), 2.10 (d, $\left.J=13.0 \mathrm{~Hz}, 1 \mathrm{H}, \mathrm{CH}_{2}\right), 1.74$ (s, 3H, CMe), 1.58 (s, 9H, CMe $), 1.40-1.33$ (m, 6H, $\left.\mathrm{CHMe} e_{2}\right), 1.30$ (m, 6H, CMe and $\mathrm{CHMe} 2$ ), 1.26-1.16 (m, 9H, CMe and $\mathrm{CHMe}$ ), 1.14-1.06 (m, 6H, CHMe $), 1.00(\mathrm{br}, 3 \mathrm{H}, \mathrm{CHMe}) .{ }^{13} \mathrm{C}\left\{{ }^{1} \mathrm{H}\right\}$ NMR $\left(\mathrm{C}_{6} \mathrm{D}_{6}, 125 \mathrm{MHz}, \mathrm{ppm}\right): \delta=307.5$ (carbene $C$ ), 179.4 (imine $C$ ), 147.0, 146.7, 146.3, 137.8, 132.9, 132.8, 128.2, 124.4, 124.2, 122.7, 122.4, $121.9(\mathrm{ArC}), 78.5\left(C_{\mathrm{q}}\right), 73.6\left(C_{\mathrm{q}}\right), 50.7\left(\mathrm{CH}_{2}\right), 45.6\left(C_{\mathrm{q}}\right), 29.8\left(\mathrm{CMe}_{3}\right), 29.4$, $29.2,29.1,28.8,27.9,27.4,27.0,26.2,23.4,23.3,22.7,22.6,22.5,21.9$.

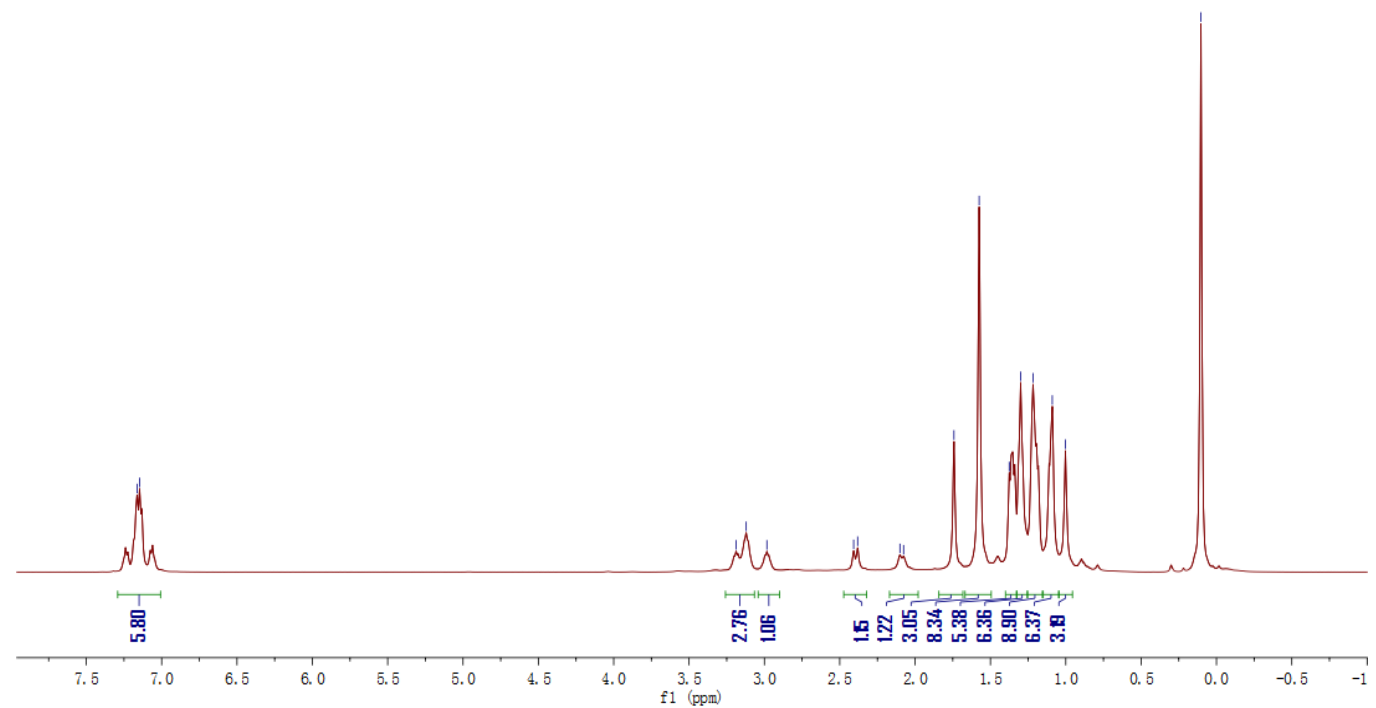

Figure S34. ${ }^{1} \mathrm{H}$ NMR of $6 \mathbf{e}\left(\mathrm{C}_{6} \mathrm{D}_{6}, 500 \mathrm{MHz}\right)$. 


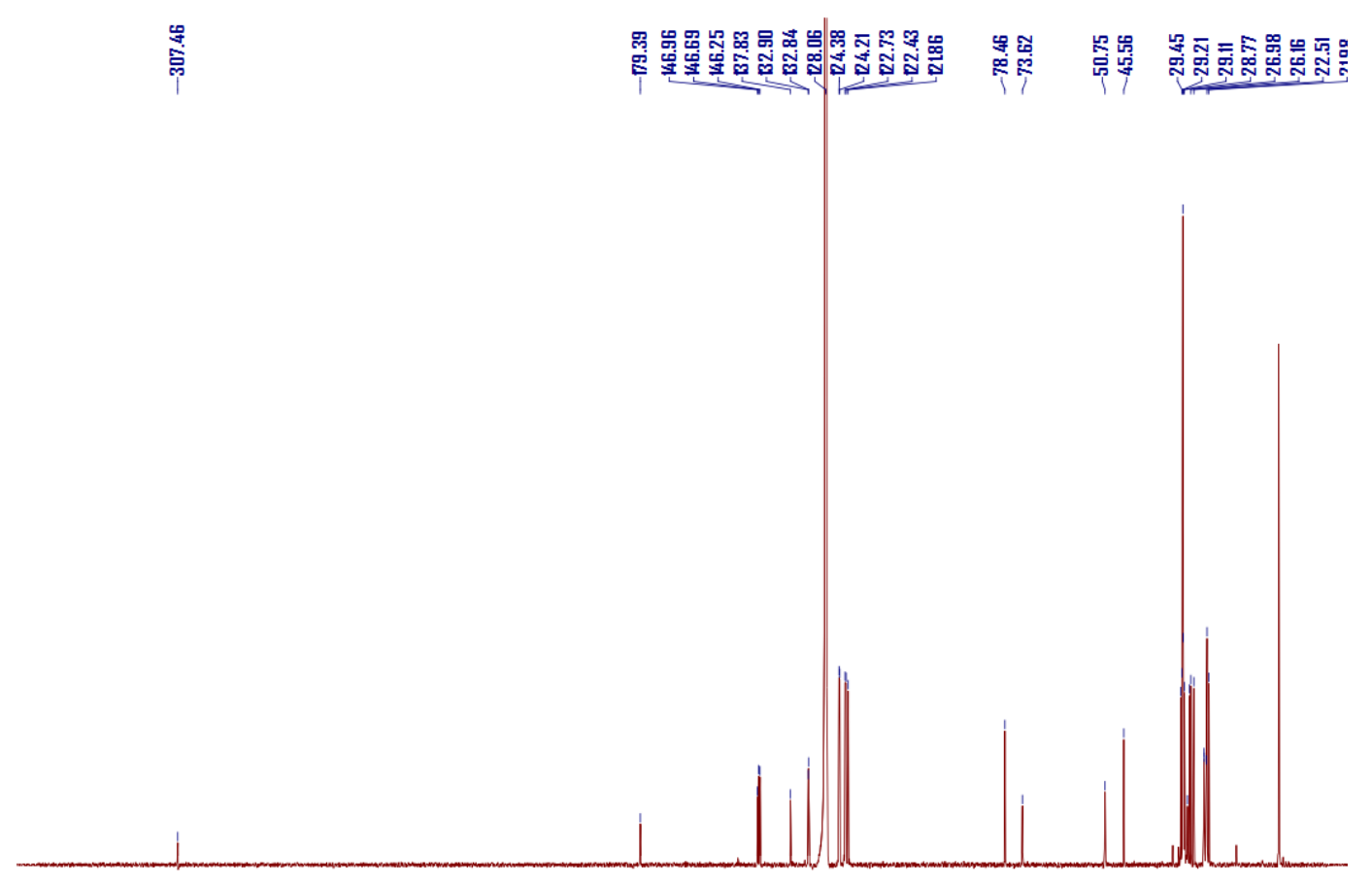

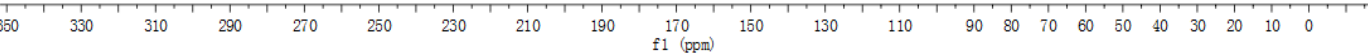

Figure S35. ${ }^{13} \mathrm{C}\left\{{ }^{1} \mathrm{H}\right\} \mathrm{NMR}$ of $6 \mathrm{e}\left(\mathrm{C}_{6} \mathrm{D}_{6}, 125 \mathrm{MHz}\right)$.

\section{Carbene 6f}

$\mathrm{C}_{6} \mathrm{D}_{6}$ was added to a mixture of cyclic iminium salt $\mathbf{4 f}(10.8 \mathrm{mg}, 0.02$ mmol) and KHMDS (4.0 mg, $0.02 \mathrm{mmol})$. It was agitated for $1 \mathrm{~min}$ and the NMR spectra were taken after $25 \mathrm{~min}$; after $1 \mathrm{~h}$ about $25 \%$ of the carbene had decomposed.

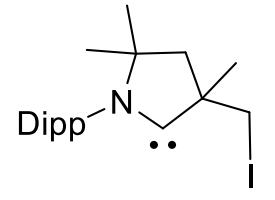

$6 f$

${ }^{1} \mathrm{H}$ NMR $\left(\mathrm{C}_{6} \mathrm{D}_{6}, 300 \mathrm{MHz}, \mathrm{ppm}\right): \delta=7.19(\mathrm{~m}, 1 \mathrm{H}, \mathrm{p}-H), 7.11(\mathrm{~m}, 2 \mathrm{H}, \mathrm{m}-H), 3.57(\mathrm{~d}, J=9.2$ $\left.\mathrm{Hz}, 1 \mathrm{H}, \mathrm{CH}_{2}\right), 3.49$ (d, $J=9.2 \mathrm{~Hz}, 1 \mathrm{H}, \mathrm{CH}_{2}$ ), 3.21 (sept, $1 \mathrm{H}, J=6.4 \mathrm{~Hz}, \mathrm{CHMe}$ ), 3.00 (sept, $\left.J=6.4 \mathrm{~Hz}, 1 \mathrm{H}, \mathrm{C} H \mathrm{Me}_{2}\right), 1.86\left(\mathrm{~d}, J=13.2 \mathrm{~Hz}, 1 \mathrm{H}, \mathrm{CH}_{2}\right), 1.42(\mathrm{~d}, J=13.2 \mathrm{~Hz}, 1 \mathrm{H}, \mathrm{CH}), 1.26$ (bs, 3H, CMe), 1.23 (bs, 3H, CMe), 1.20 (bs, 3H, CMe), 1.18 (bs, 6H), 1.16 (bs, 3H), 1.03 (bs, $3 \mathrm{H}), 1.0$ (bs, 3H). ${ }^{13} \mathrm{C}\left\{{ }^{1} \mathrm{H}\right\} \mathrm{NMR}\left(\mathrm{C}_{6} \mathrm{D}_{6}, 125 \mathrm{MHz}, \mathrm{ppm}\right): \delta=307.1$ (carbene), 146.6, 146.2, 137.6, 124.8, 124.4, 124.3 ( $\mathrm{ArC}), 83.5\left(\mathrm{C}_{\mathrm{q}}\right), 62.5,48.4,29.9,29.6,29.1,28.8,28.7,27.1,26.7$, $22.3,22.1,7.65\left(\mathrm{CH}_{2} \mathrm{I}\right)$. 


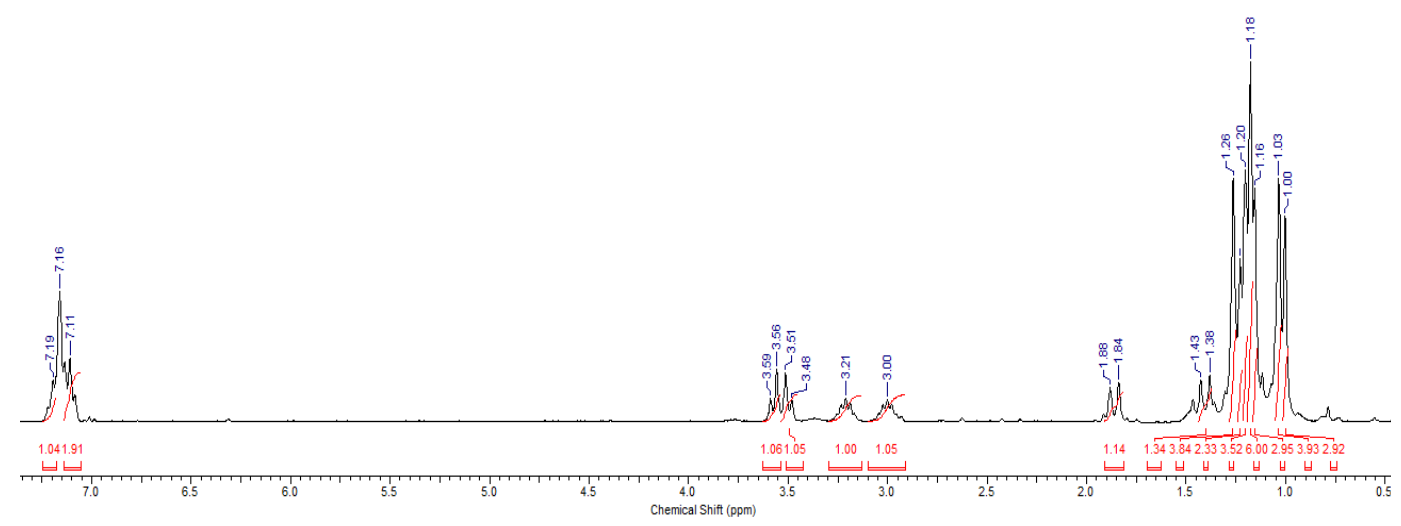

Figure S36. ${ }^{1} \mathrm{H}$ NMR of $\mathbf{6 f}\left(\mathrm{C}_{6} \mathrm{D}_{6}, 300 \mathrm{MHz}\right)$.

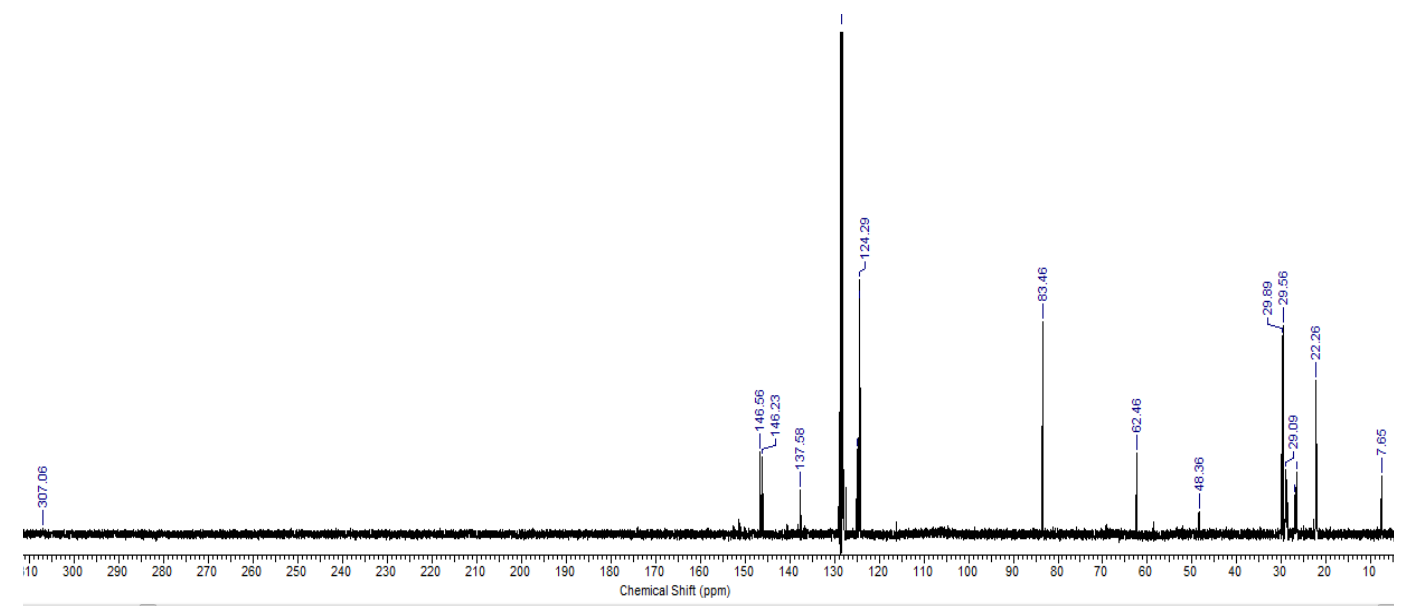

Figure S37. ${ }^{13} \mathrm{C}\left\{{ }^{1} \mathrm{H}\right\}$ NMR of $6 \mathbf{f}\left(\mathrm{C}_{6} \mathrm{D}_{6}, 125 \mathrm{MHz}\right)$.

\section{Carbene 6g}

$\mathrm{C}_{6} \mathrm{D}_{6}$ was added to a mixture of cyclic iminium salt $4 \mathrm{~g}$ (15 mg, 0.025 mmol) and KHMDS ( $5 \mathrm{mg}, 0.025 \mathrm{mmol}$ ) in glovebox. It was agitated for $1 \mathrm{~min}$ and the NMR was taken after $10 \mathrm{~min}$.

${ }^{1} \mathrm{H}$ NMR $\left(\mathrm{C}_{6} \mathrm{D}_{6}, 500 \mathrm{MHz}, \mathrm{ppm}\right): \delta=7.90-7.70(\mathrm{~m}, 4 \mathrm{H}, \mathrm{Ar} H)$, 7.40-7.10 (m, 9H, $\mathrm{Ar} H$ ), 3.71 (sept, $J=7.0 \mathrm{~Hz}, 1 \mathrm{H}, \mathrm{CHMe}$ ), 3.31

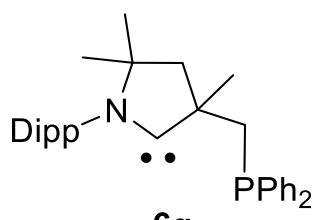
(sept, $J=7.0 \mathrm{~Hz}, 1 \mathrm{H}, \mathrm{C} H \mathrm{Me}_{2}$ ), $3.12(\mathrm{dd}, J=14.0 \mathrm{~Hz}, J=3.5 \mathrm{~Hz}, 1 \mathrm{H}, \mathrm{PCH}$ ), 2.93 (dd, $J=$ $\left.14.0 \mathrm{~Hz}, J=3.5 \mathrm{~Hz}, 1 \mathrm{H}, \mathrm{PCH} \mathrm{H}_{2}\right), 2.11\left(\mathrm{~d}, J=13.0 \mathrm{~Hz}, 1 \mathrm{H}, \mathrm{CH}_{2}\right), 1.61(\mathrm{~s}, 3 \mathrm{H}, \mathrm{CMe}), 1.45$ (d, $J$ $\left.=7.0 \mathrm{~Hz}, 3 \mathrm{H}, \mathrm{CH} M e_{2}\right), 1.40\left(\mathrm{~d}, J=7.0 \mathrm{~Hz}, 3 \mathrm{H}, \mathrm{CH} M e_{2}\right), 1.39$ (d, $\left.J=7.0 \mathrm{~Hz}, 3 \mathrm{H}, \mathrm{CH} M e_{2}\right)$, $1.30\left(\mathrm{~d}, J=7.0 \mathrm{~Hz}, 3 \mathrm{H}, \mathrm{CH} M e_{2}\right), 1.29$ (s, 3H, CMe), 1.23 (s, 3H, CMe). ${ }^{13} \mathrm{C}\left\{{ }^{1} \mathrm{H}\right\} \mathrm{NMR}$ $\left(\mathrm{C}_{6} \mathrm{D}_{6}, 125 \mathrm{MHz}, \mathrm{ppm}\right): \delta=309.7(\mathrm{~d}, J=9.9 \mathrm{~Hz}$, iminium $C), 146.7,146.0,142.0(\mathrm{~d}, J=13.1$ $\mathrm{Hz}), 141.3(\mathrm{~d}, J=13.4 \mathrm{~Hz}), 137.8,134.3,134.2,133.0,132.8,124.2,123.8(\operatorname{ArC}), 82.8\left(\mathrm{C}_{\mathrm{q}}\right)$, $62.1(\mathrm{~d}, J=4.2 \mathrm{~Hz}), 49.1(\mathrm{~d}, J=10.1 \mathrm{~Hz}), 43.2(\mathrm{~d}, J=14.1 \mathrm{~Hz}), 29.6(\mathrm{~d}, J=5.4 \mathrm{~Hz}), 29.3$, 29.0, 28.6, 28.0, 27.9, 27.0, 26.5, 22.0 (d, $J=4.0 \mathrm{~Hz}) .{ }^{31} \mathrm{P}$ NMR $\left(\mathrm{C}_{6} \mathrm{D}_{6}, 121 \mathrm{MHz}\right.$, ppm):-19.7. 


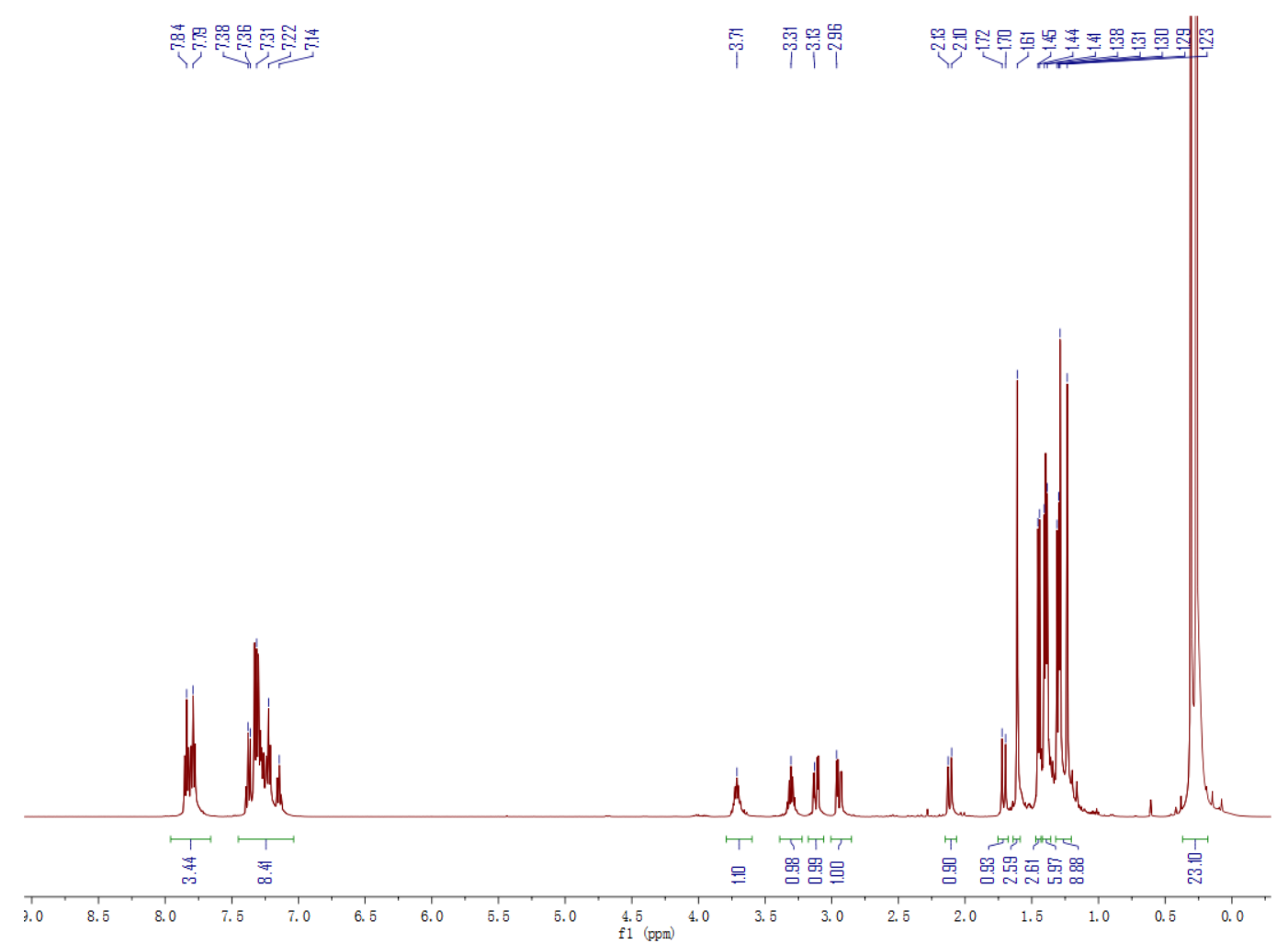

Figure S38. ${ }^{1} \mathrm{H}$ NMR of $\mathbf{6 g}\left(\mathrm{C}_{6} \mathrm{D}_{6}, 500 \mathrm{MHz}\right)$.

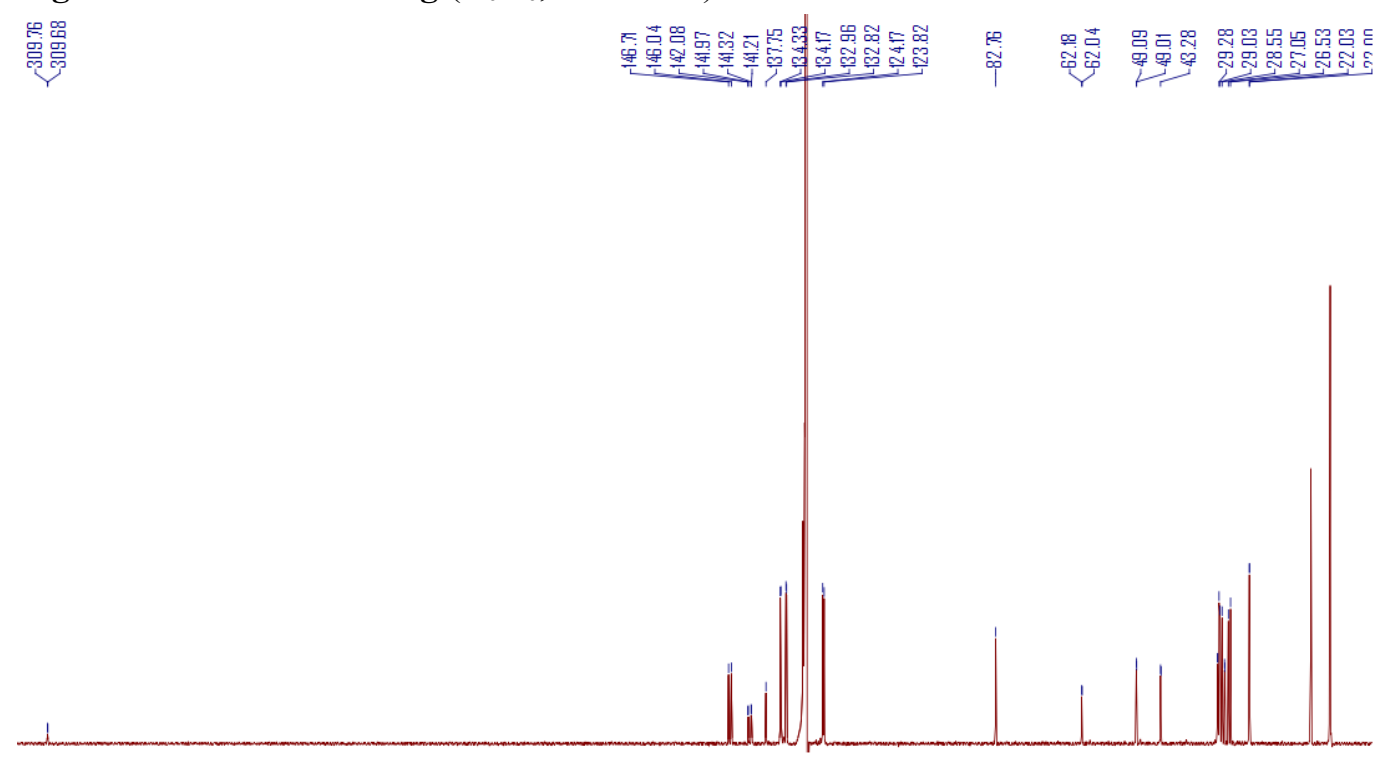

$\begin{array}{llllllllllllllllllllllllllllllllllll}310 & 300 & 290 & 280 & 270 & 260 & 250 & 240 & 230 & 220 & 210 & 200 & 190 & 18 & 170 & 160 & 150 & 140 & 130 & 120 & 110 & 100 & 90 & 80 & 70 & 60 & 50 & 40 & 30 & 20 & 10 & 0\end{array}$

Figure S39. ${ }^{13} \mathrm{C}\left\{{ }^{1} \mathrm{H}\right\}$ NMR of $6 \mathbf{g}\left(\mathrm{C}_{6} \mathrm{D}_{6}, 125 \mathrm{MHz}\right)$. 


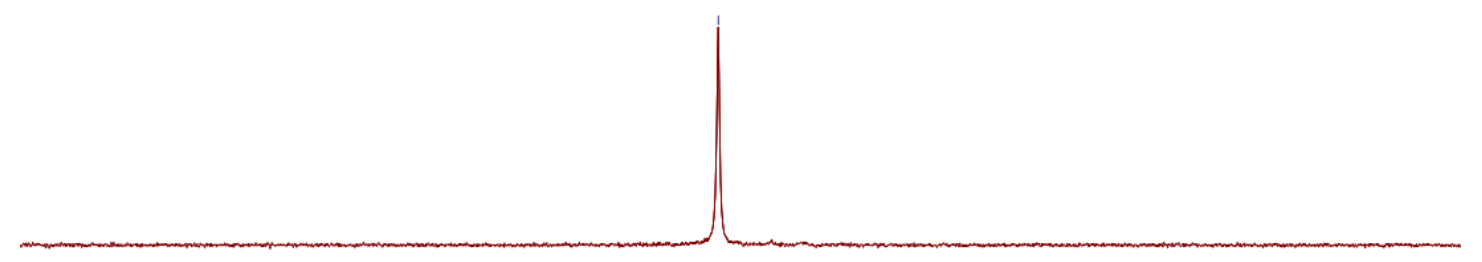

Figure S40. ${ }^{31} \mathrm{P}$ NMR of $\mathbf{6 g}\left(\mathrm{C}_{6} \mathrm{D}_{6}, 121 \mathrm{MHz}\right)$.

\section{Copper and Gold Complexes}

\section{Copper complex (6a)CuCl}

Cyclic iminium salt $4 \mathbf{a}(415 \mathrm{mg}, 0.87 \mathrm{mmol})$ was put in a Schlenk flask together with 1 eq of KHMDS (174 mg). THF $(10 \mathrm{~mL})$ was added and it was stirred for $5 \mathrm{~min}$, during which a colorless precipitate formed. The solution was transferred via canula to another Schlenk flask, which contained 1.5 eq. of

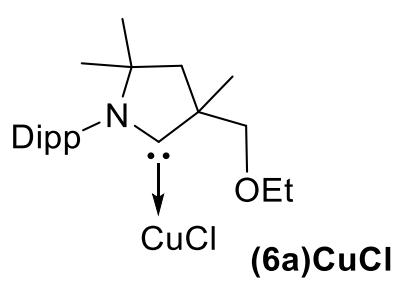

$\mathrm{CuCl}(131 \mathrm{mg})$. It was stirred over night at room temperature. The solvent was evaporated in vacuo, it was extracted with benzene, and the extract was washed after evaporation with hexanes until the solution was colorless. The residue was extracted again with $10 \mathrm{~mL}$ of $\mathrm{Et}_{2} \mathrm{O}$ and evaporation of the solution yielded an off colorless powder $(260 \mathrm{mg}, 70 \%)$. The product could also be obtained by stirring the cyclic iminium salt $4 \mathbf{a}$ in presence of 5 eq. of $\mathrm{KCl}$ and 5 eq. $\mathrm{Cu}_{2} \mathrm{O}$ at $100{ }^{\circ} \mathrm{C}$ in THF in a pressure tube for 3 days and then following the same workup (22\%). m.p. $129^{\circ} \mathrm{C}(\mathrm{dec})$.

${ }^{1} \mathrm{H}$ NMR $\left(\mathrm{C}_{6} \mathrm{D}_{6}, 400 \mathrm{MHz}, \mathrm{ppm}\right): \delta=7.15(\mathrm{t}, J=7.7 \mathrm{~Hz}, 1 \mathrm{H}, \mathrm{Ar} H), 7.05(\mathrm{~d}, J=7.7 \mathrm{~Hz}, 1 \mathrm{H}$, $\operatorname{Ar} H), 7.01(\mathrm{~d}, J=7.7 \mathrm{~Hz}, 1 \mathrm{H}, \mathrm{Ar} H), 3.74\left(\mathrm{~d}, J=8.8 \mathrm{~Hz}, 1 \mathrm{H}, \mathrm{CH}_{2}\right), 3.23(\mathrm{~m}, 1 \mathrm{H}), 3.13(\mathrm{~m}$, $1 \mathrm{H}), 3.08(\mathrm{~m}, 1 \mathrm{H}), 2.90\left(\mathrm{~d}, J=8.8 \mathrm{~Hz}, 1 \mathrm{H}, \mathrm{CH}_{2}\right), 2.73(\mathrm{~m}, 1 \mathrm{H}), 1.98(\mathrm{~d}, J=13.0 \mathrm{~Hz}, 1 \mathrm{H}$, $\left.\mathrm{CH}_{2}\right), 1.43(\mathrm{~s}, 3 \mathrm{H}), 1.41(\mathrm{~s}, 3 \mathrm{H}), 1.30\left(\mathrm{~d}, J=13.0 \mathrm{~Hz}, 3 \mathrm{H}, \mathrm{CH}_{3}\right), 1.14(\mathrm{~d}, J=6.8 \mathrm{~Hz}, 3 \mathrm{H}$, $\left.\mathrm{CH}_{3}\right), 1.11$ (d, $\left.J=6.8 \mathrm{~Hz}, 3 \mathrm{H}, \mathrm{CH}_{3}\right), 1.03$ (s, 3H), 1.00 (t, $\left.J=6.8 \mathrm{~Hz}, 3 \mathrm{H}, \mathrm{CH}_{3}\right), 0.96$ (s, 3H), $0.88(\mathrm{~s}, 3 \mathrm{H}) \cdot{ }^{13} \mathrm{C}\left\{{ }^{1} \mathrm{H}\right\} \mathrm{NMR}\left(\mathrm{C}_{6} \mathrm{D}_{6}, 125 \mathrm{MHz}, \mathrm{ppm}\right): \delta=247.5$ (carbene), 145.6, 144.9, 134.4, 
129.7, 124.7, $124.4(\mathrm{ArC}), 81.6\left(C_{\mathrm{q}}\right), 76.7\left(\mathrm{CH}_{2}\right), 66.5\left(\mathrm{CH}_{2}\right)$, 58.8, $44.0\left(\mathrm{CH}_{2}\right), 29.2,29.0$, 28.3, 27.7, 27.3, 27.0, 23.0, 22.1, 22.1, 14.9 .

HRMS: $\mathrm{m} / \mathrm{z}$ calculated for $\left[\mathrm{C}_{22} \mathrm{H}_{35} \mathrm{CuNO}\right]^{+} 392.2015$ for found 392.2013.

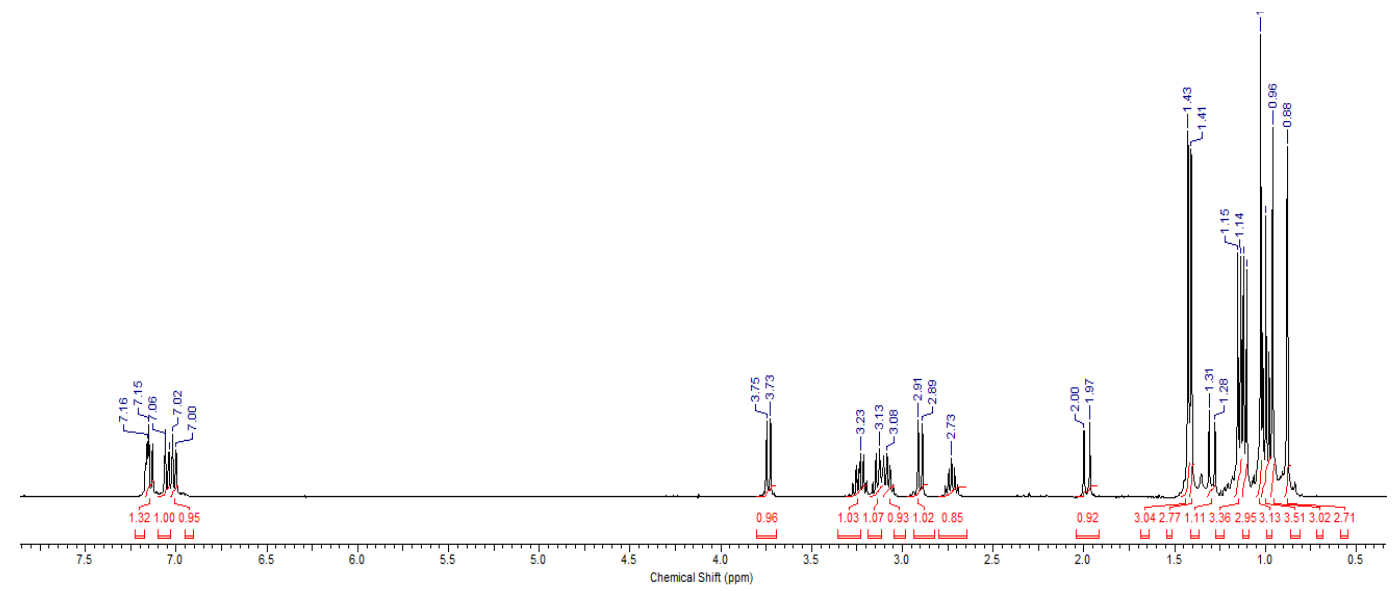

Figure S41. ${ }^{1} \mathrm{H} \mathrm{NMR}$ of (6a)CuCl $\left(\mathrm{C}_{6} \mathrm{D}_{6}, 400 \mathrm{MHz}\right)$.

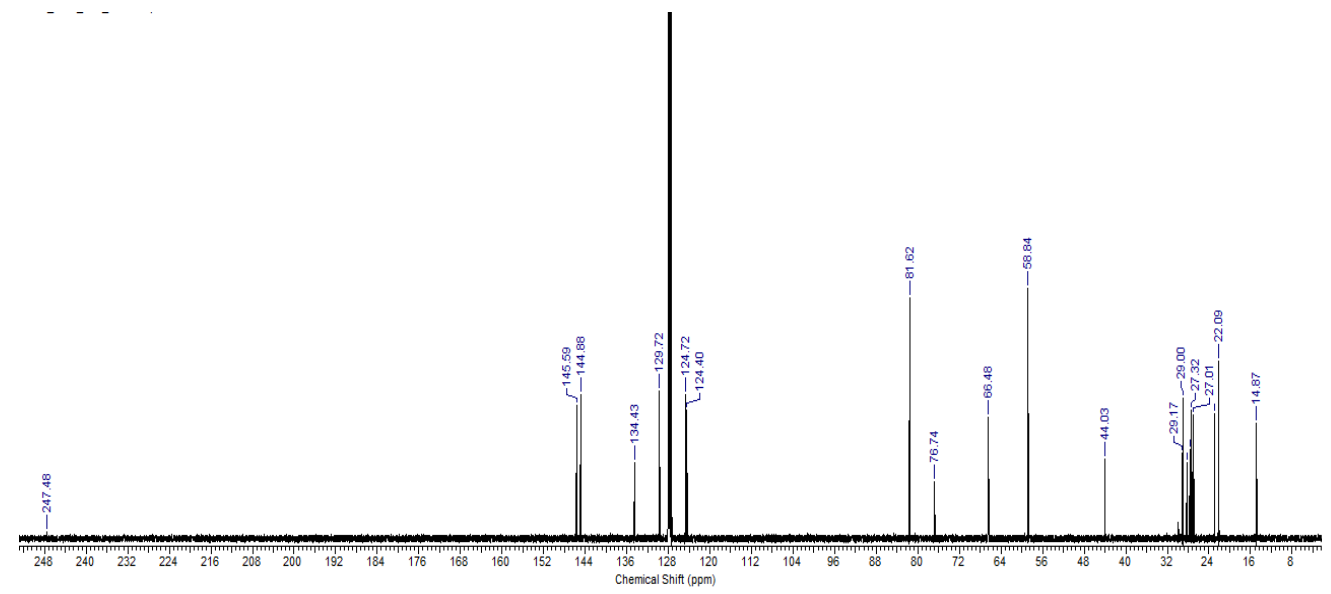

Figure S42. ${ }^{13} \mathrm{C}\left\{{ }^{1} \mathrm{H}\right\} \mathrm{NMR}$ of (6a)CuCl $\left(\mathrm{C}_{6} \mathrm{D}_{6}, 125 \mathrm{MHz}\right)$.

\section{Copper complex (6b)CuCl}

THF $(20 \mathrm{~mL})$ was added to a mixture of cyclic iminium salt $\mathbf{4 b}$ (0.41 g, $1.0 \mathrm{mmol})$ and KHMDS (0.21 g, $1.05 \mathrm{mmol})$ under stirring at $-20{ }^{\circ} \mathrm{C}$, and the reaction mixture was stirred at room temperature for 30 minutes. After the solvent was removed in vacuo, the free carbene was extracted with ether $(20 \mathrm{~mL})$. The

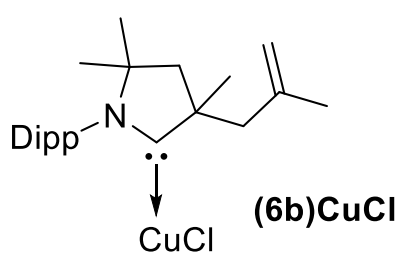
ether solution was filtered to another schlenk flask and $\mathrm{CuCl}$ $(0.12 \mathrm{~g}, 1.2 \mathrm{mmol})$ was added at $0{ }^{\circ} \mathrm{C}$. The reaction mixture was stirred at room temperature for 3 hours. After ether was removed in vacuo, the solid was extracted with benzene (60 $\mathrm{mL})$. After benzene was removed in vacuo, the solid was washed with hexane $(50 \mathrm{~mL})$ to afford a yellow powder $(0.25 \mathrm{~g}, 59 \%)$. m.p. $149{ }^{\circ} \mathrm{C}(\mathrm{dec})$.

${ }^{1} \mathrm{H}$ NMR $\left(\mathrm{C}_{6} \mathrm{D}_{6}, 400 \mathrm{MHz}\right): \delta=7.11(\mathrm{t}, J=7.2 \mathrm{~Hz}, 1 \mathrm{H}, p-\mathrm{H}), 6.98(\mathrm{~d}, J=7.2 \mathrm{~Hz}, 2 \mathrm{H}, m-\mathrm{H})$, $4.85\left(\mathrm{~m}, 2 \mathrm{H},=\mathrm{CH}_{2}\right), 2.70$ (sept, $\left.J=13.6 \mathrm{~Hz}, 1 \mathrm{H}, \mathrm{CH}_{2}\right), 2.46\left(\mathrm{~d}, J=13.6 \mathrm{~Hz}, 1 \mathrm{H}, \mathrm{CH}_{2}\right), 1.84$ $\left(\mathrm{d}, J=13.2 \mathrm{~Hz}, 1 \mathrm{H}, \mathrm{CH}_{2}\right), 1.73\left(\mathrm{~s}, 3 \mathrm{H}, \mathrm{CH}_{3}\right), 1.43(\mathrm{~d}, J=6.8 \mathrm{~Hz}, 3 \mathrm{H}, \mathrm{CHMe}), 1.36(\mathrm{~d}, J=$ 
$6.8 \mathrm{~Hz}, 3 \mathrm{H}, \mathrm{CHMe}$ ) 1.28 (d, $J=13.2 \mathrm{~Hz}, 1 \mathrm{H}, \mathrm{CH}_{2}$ ), 1.13 (s, 3H, CMe), 1.07 (d, $J=6.8 \mathrm{~Hz}$, $3 \mathrm{H}, \mathrm{CHMe}$ ), $1.06(\mathrm{~d}, J=7.0 \mathrm{~Hz}, 3 \mathrm{H}, \mathrm{CHMe}), 0.81(\mathrm{~s}, 3 \mathrm{H}, \mathrm{CMe}), 0.80(\mathrm{~s}, 3 \mathrm{H}, \mathrm{CMe}) .{ }^{13} \mathrm{C}\left\{{ }^{1} \mathrm{H}\right\}$ NMR $\left(\mathrm{C}_{6} \mathrm{D}_{6}, 125 \mathrm{MHz}\right): \delta=249.0$ (carbene $C$ ), 145.4, 145.3, 142.1, 134.3, 130.1, 125.0, 124.9, 116.4 ( $\mathrm{ArC}$ and $\left.C=\mathrm{CH}_{2}\right), 80.7\left(C_{\mathrm{q}}\right), 58.3\left(\mathrm{C}_{\mathrm{q}}\right), 48.5\left(\mathrm{CH}_{2}\right), 45.7\left(\mathrm{CH}_{2}\right), 29.4,29.2,28.9$, 28.4, 27.5, 27.3, 27.2, 25.4, 22.5, 22.3. HRMS calculated for $\left[\mathrm{C}_{23} \mathrm{H}_{35} \mathrm{Cu} \mathrm{N}\right]^{+} 388.2060$ found 388.2059 .

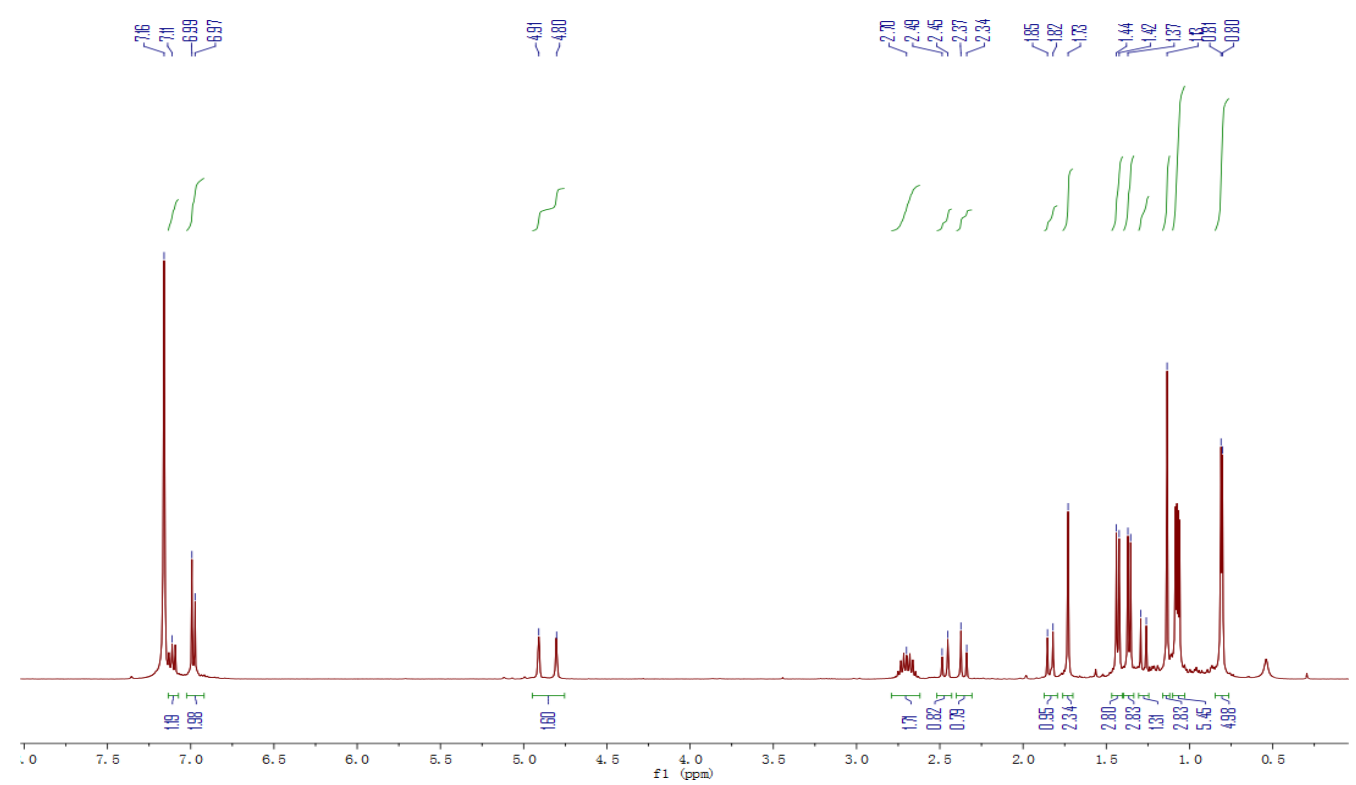

Figure S43. ${ }^{1} \mathrm{H}$ NMR of (6b)CuCl $\left(\mathrm{C}_{6} \mathrm{D}_{6}, 400 \mathrm{MHz}\right)$.

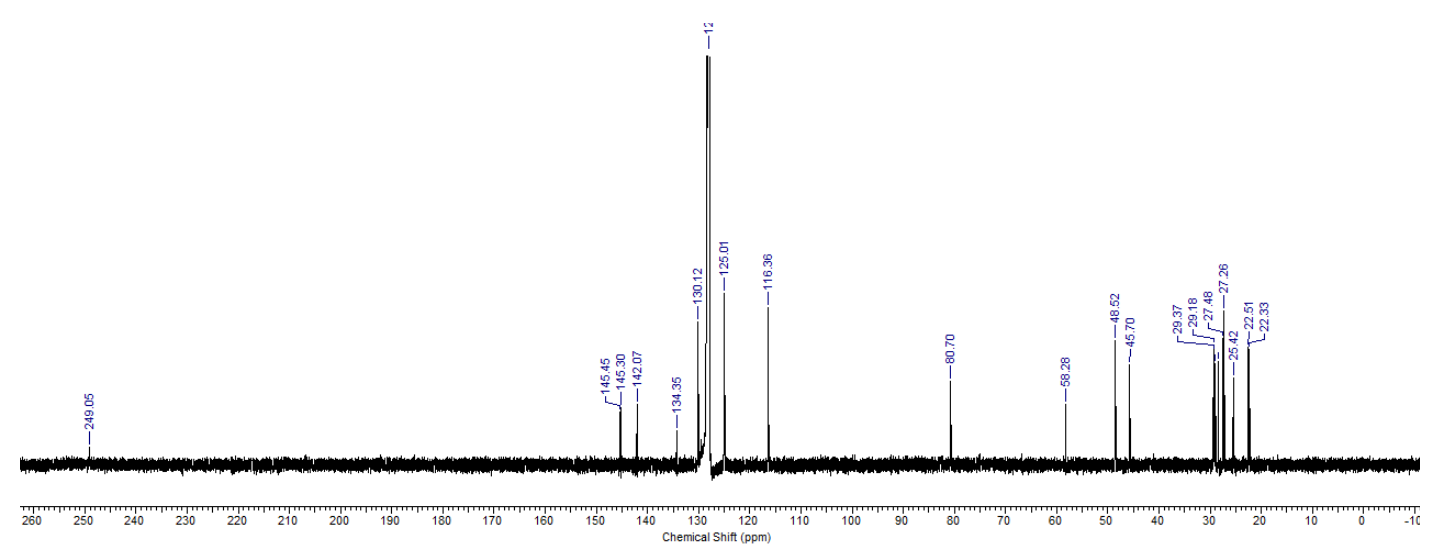

Figure S44. ${ }^{13} \mathrm{C}\left\{{ }^{1} \mathrm{H}\right\}$ NMR of (6b)CuCl $\left(\mathrm{C}_{6} \mathrm{D}_{6}, 125 \mathrm{MHz}\right)$.

\section{Copper chloride complex (6d)CuCl}

THF $(20 \mathrm{~mL})$ was added to a mixture of cyclic iminium salt $\mathbf{4 d}$ $(0.83 \mathrm{~g}, 2.0 \mathrm{mmol})$ and KHMDS $(0.40 \mathrm{~g}, 2.0 \mathrm{mmol})$ and $\mathrm{CuCl}$ $(0.24 \mathrm{~g}, 2.4 \mathrm{mmol})$ under stirring at $-78{ }^{\circ} \mathrm{C}$, and the reaction mixture was slowly warmed to room temperature in 12 hours. After the solvent was removed in vacuo, the crude product was extracted with benzene $(40 \mathrm{~mL})$. After benzene was removed in

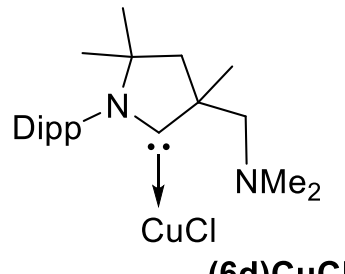

(6d) $\mathrm{CuCl}$ 
vacuo, the pale yellow solid was washed with hexane $(2 \times 40 \mathrm{~mL})$ to afford a pale yellow powder $(0.66 \mathrm{~g}, 77 \%)$. m.p. $186-188^{\circ} \mathrm{C}$ (dec.).

${ }^{1} \mathrm{H}$ NMR $\left(\mathrm{C}_{6} \mathrm{D}_{6}, 500 \mathrm{MHz}, \mathrm{ppm}\right): \delta=7.12(\mathrm{t}, J=8.0 \mathrm{~Hz}, 1 \mathrm{H}, p-\mathrm{H}), 7.03-6.97(\mathrm{~m}, 2 \mathrm{H}, m-\mathrm{H})$, 2.96 (sept, $J=7.0 \mathrm{~Hz}, 1 \mathrm{H}, \mathrm{C} H \mathrm{Me}_{2}$ ), $2.83\left(\mathrm{~d}, J=14.0 \mathrm{~Hz}, 1 \mathrm{H}, \mathrm{CH}_{2}\right.$ ), 2.70 (sept, $J=7.0 \mathrm{~Hz}$, $1 \mathrm{H}, \mathrm{CHMe}_{2}$ ), 2.20 (s, 6H, NMe $\left.e_{2}\right), 2.01$ (d, $\left.J=14.0 \mathrm{~Hz}, 2 \mathrm{H}, \mathrm{CH}_{2}\right), 1.43$ (d, $J=7.0 \mathrm{~Hz}, 3 \mathrm{H}$, $\mathrm{CHMe}$ ), 1.37 (d, $J=7.0 \mathrm{~Hz}, 3 \mathrm{H}, \mathrm{CHMe}$ ) 1.20 (d, $\left.J=13.0 \mathrm{~Hz}, 1 \mathrm{H}, \mathrm{CH}_{2}\right), 1.11$ (d, $J=7.0 \mathrm{~Hz}$, $3 \mathrm{H}, \mathrm{CHMe}_{2}$ ), 1.09 (d, J=7.0 Hz, 3H, CHMe $\left.e_{2}\right), 0.99$ (s, 3H, CMe), 0.94 (s, 3H, CMe), 0.85 (s, $3 \mathrm{H}, \mathrm{CMe}) .{ }^{13} \mathrm{C}\left\{{ }^{1} \mathrm{H}\right\}$ NMR $\left(\mathrm{C}_{6} \mathrm{D}_{6}, 125 \mathrm{MHz}, \mathrm{ppm}\right): \delta=248.4$ (carbene $C$ ), 145.5, 145.4, 134.7, 130.0, 124.9, 124.8, $(\mathrm{ArC}), 81.1\left(C_{\mathrm{q}}\right), 68.7\left(\mathrm{CH}_{2}\right), 60.8\left(C_{\mathrm{q}}\right), 48.6\left(\mathrm{NMe}_{2}\right), 44.3\left(\mathrm{CH}_{2}\right), 29.2$, 29.0, 28.8, 28.7, 27.9, 27.3, 25.2, 22.5. HRMS: $\mathrm{m} / \mathrm{z}$ calculated for $\left[\mathrm{C}_{22} \mathrm{H}_{36} \mathrm{CuN}_{2}\right]^{+} \mathrm{M}+$ 391.2169 , found 391.2169 .

못루후용

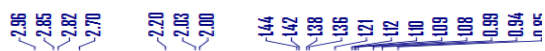

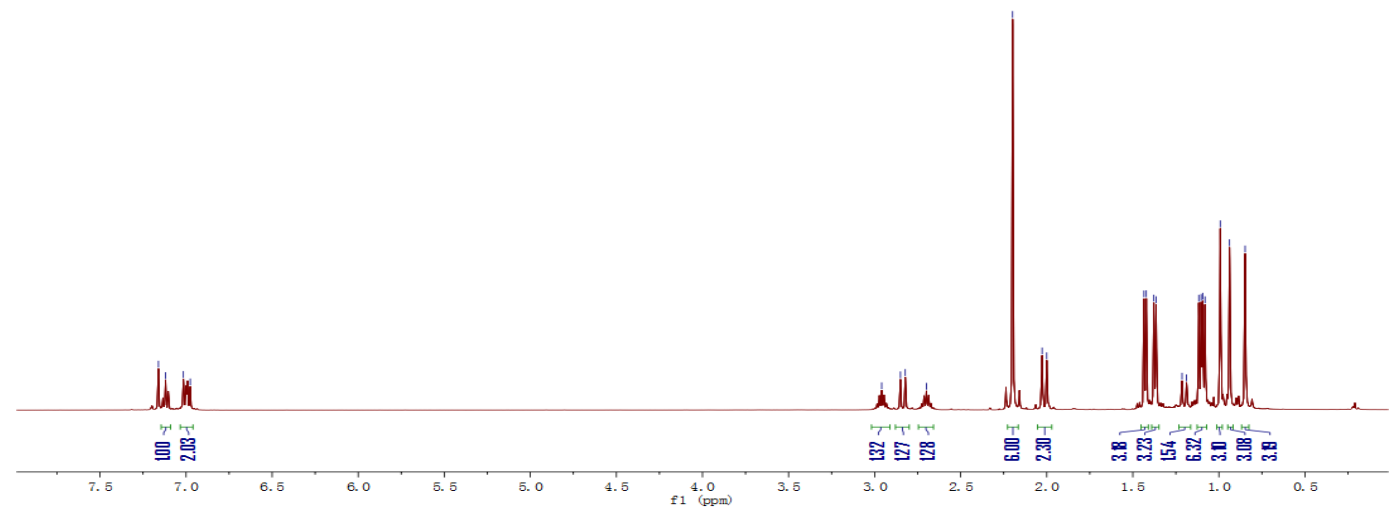

Figure S45. ' $\mathrm{H}$ NMR of (6d) CuCl $\left(\mathrm{C}_{6} \mathrm{D}_{6}, 500 \mathrm{MHz}\right)$. 


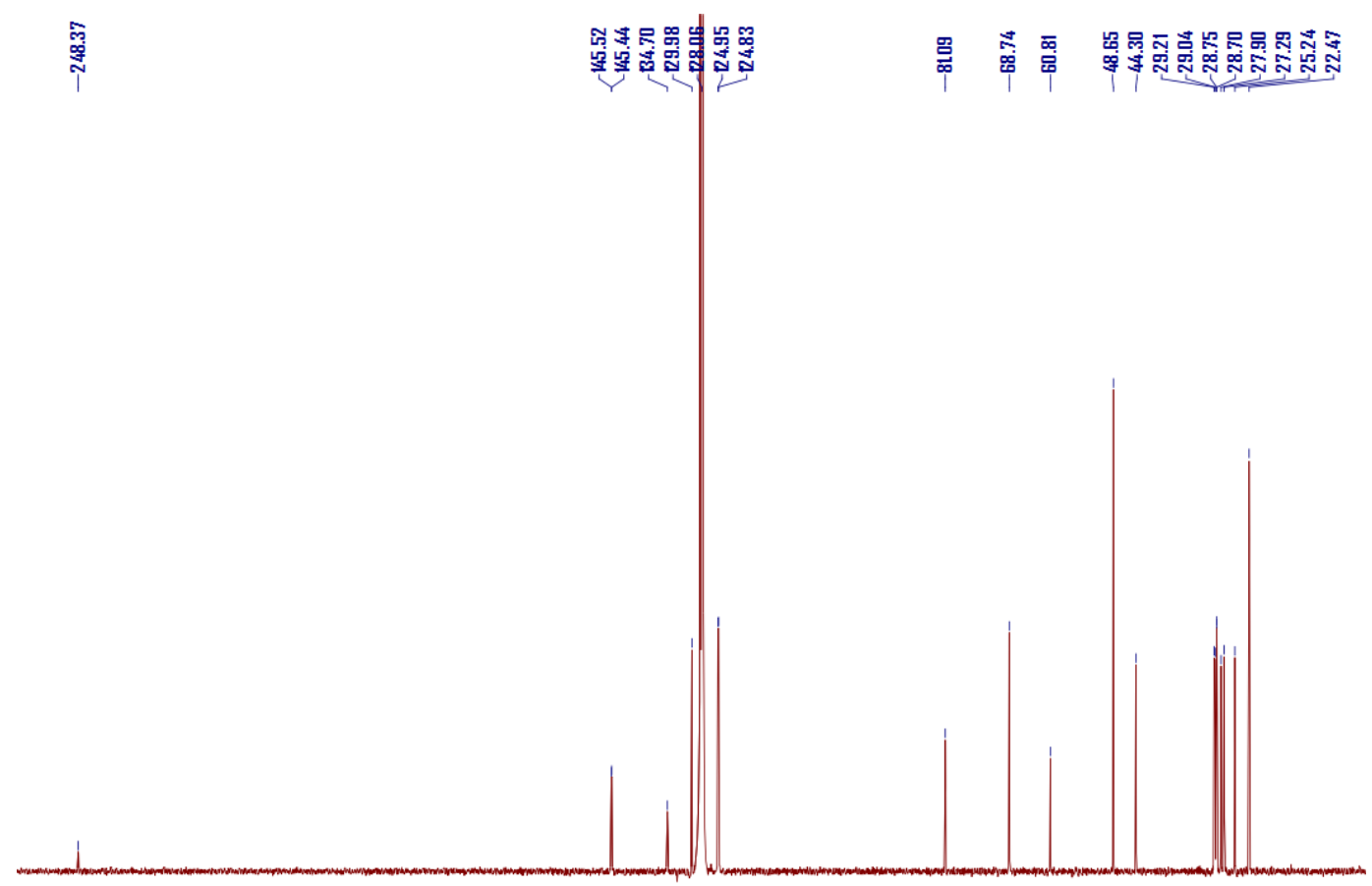

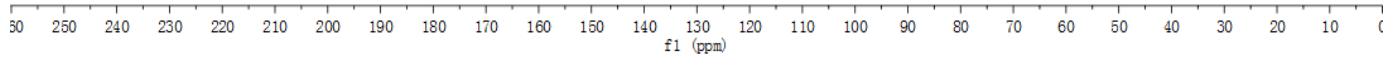

Figure S46. ${ }^{13} \mathrm{C}\left\{{ }^{1} \mathrm{H}\right\} \mathrm{NMR}$ of (6d) CuCl $\left(\mathrm{C}_{6} \mathrm{D}_{6}, 125 \mathrm{MHz}\right)$.

\section{Gold chloride complex (6d)AuCI}

THF $(20 \mathrm{~mL})$ was added to a mixture of cyclic iminium salt $\mathbf{4 d}$ (832 $\mathrm{mg}, 2.0 \mathrm{mmol})$, KHMDS (399 $\mathrm{mg}, 2.0 \mathrm{mmol})$ and $\mathrm{AuCl}(\mathrm{THT})(641 \mathrm{mg}, 2.0 \mathrm{mmol})$ under stirring at $-78^{\circ} \mathrm{C}$, and the reaction mixture was slowly warmed to room temperature over 12 hours. After the solvent was removed in vacuo, the crude product

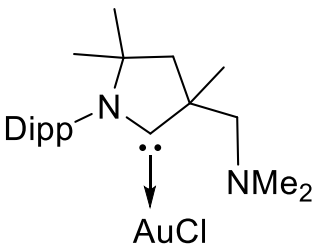

(6d)AuCI was extracted with benzene $(2 \times 25 \mathrm{~mL})$. Then benzene was removed in vacuo and the solid was washed with hexane $(2 \times 40 \mathrm{~mL})$ to afford a yellow powder $(930 \mathrm{mg}, 83 \%)$. m.p. 192-194 ${ }^{\circ} \mathrm{C}$ (dec.).

${ }^{1} \mathrm{H}$ NMR $\left(\mathrm{C}_{6} \mathrm{D}_{6}, 300 \mathrm{MHz}, \mathrm{ppm}\right): \delta=7.11(\mathrm{t}, J=7.5 \mathrm{~Hz}, 1 \mathrm{H}, p-\mathrm{H}), 6.97(\mathrm{~m}, 2 \mathrm{H}, m-\mathrm{H})$, 3.00-2.90 (m, 2H, CH$H_{2}$ and $\mathrm{CHMe}$ ), 2.66 (sept, $J=6.9 \mathrm{~Hz}, 1 \mathrm{H}, \mathrm{CHMe}$ ), $2.20\left(\mathrm{~s}, 6 \mathrm{H}, \mathrm{NMe} \mathrm{e}_{2}\right.$ ), $2.06\left(\mathrm{~d}, J=13.2 \mathrm{~Hz}, 1 \mathrm{H}, \mathrm{CH}_{2}\right), 1.92\left(\mathrm{~d}, J=14.1 \mathrm{~Hz}, 1 \mathrm{H}, \mathrm{CH}_{2}\right), 1.52$ (d, $J=6.9 \mathrm{~Hz}, 3 \mathrm{H}$, $\mathrm{CHMe}_{2}$ ), 1.44 (d, J=6.9 Hz, 3H, CHMe $), 1.24$ (d, J=12.9 Hz, 1H, CH $), 1.10$ (d, J=6.9 Hz, 3H, CHMe $), 1.08$ (d, J=6.9 Hz, 3H, CHMe $), 1.03$ (s, 3H, CMe), 0.94 (s, 3H, CMe), 0.84 (s, $3 \mathrm{H}, \mathrm{CMe}) .{ }^{13} \mathrm{C}\left\{{ }^{1} \mathrm{H}\right\}$ NMR $\left(\mathrm{C}_{6} \mathrm{D}_{6}, 125 \mathrm{MHz}, \mathrm{ppm}\right): \delta=236.2$ (carbene $\left.C\right), 145.5,145.4,134.4$, 130.1, 125.0, $124.9(\mathrm{ArC}), 80.5\left(C_{\mathrm{q}}\right), 68.3\left(\mathrm{CH}_{2}\right), 60.6\left(C_{\mathrm{q}}\right), 48.7\left(\mathrm{NMe}_{2}\right), 44.2\left(\mathrm{CH}_{2}\right), 29.3$, $29.0,28.9,28.6,27.7,27.2,26.2,22.9,22.8$. HRMS: $\mathrm{m} / \mathrm{z}$ calculated for $\left[\mathrm{C}_{24} \mathrm{H}_{39} \mathrm{AuN}_{3}\right]^{+}$ 566.2804 , found 566.2802 (acetonitrile complex in solvent acetonitrile). 


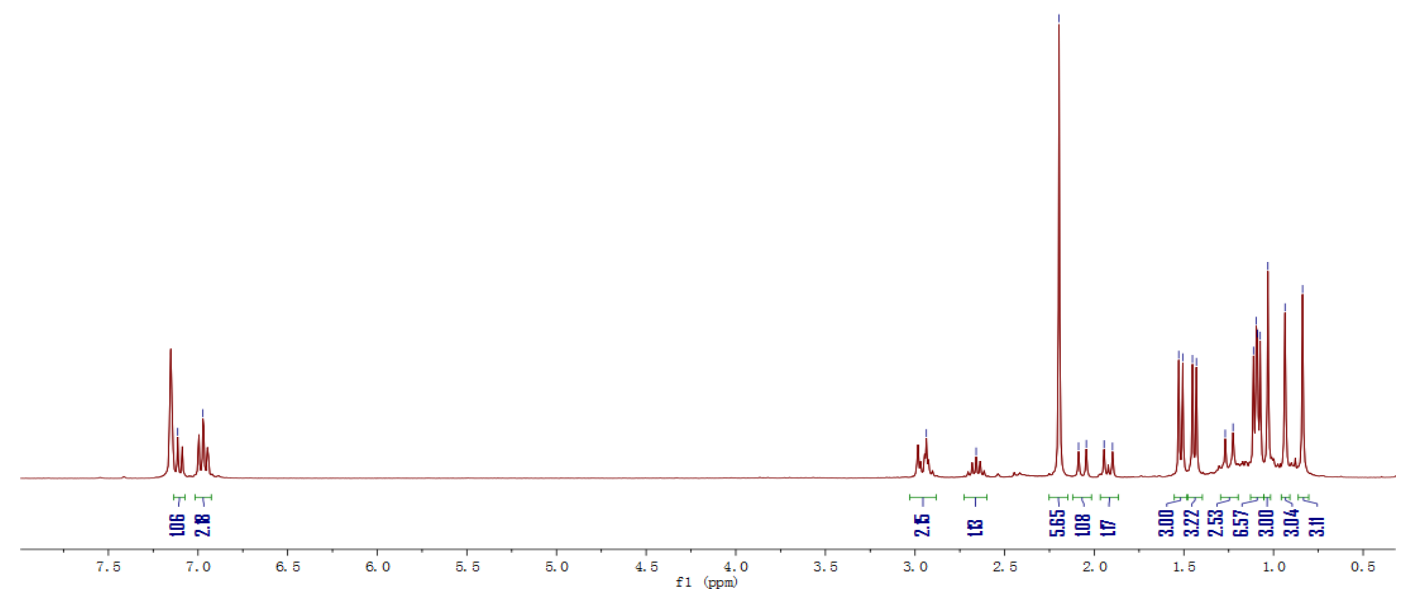

Figure S47. 'H NMR of (6d)AuCl $\left(\mathrm{C}_{6} \mathrm{D}_{6}, 300 \mathrm{MHz}\right)$.

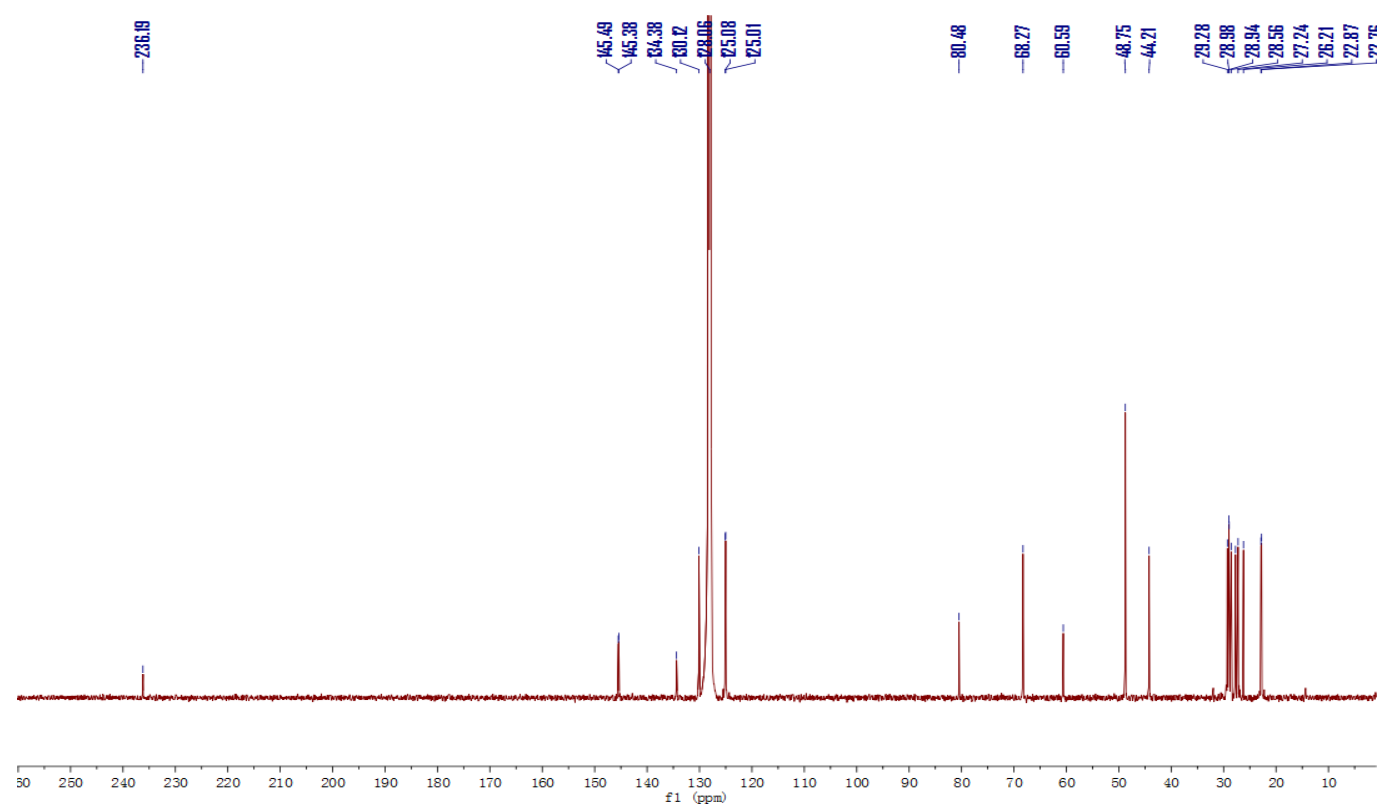

Figure S48. ${ }^{13} \mathrm{C}\left\{{ }^{1} \mathrm{H}\right\}$ NMR of (6d)AuCl $\left(\mathrm{C}_{6} \mathrm{D}_{6}, 125 \mathrm{MHz}\right)$.

\section{Copper chloride complex (6e) $\mathbf{C u C l}$}

THF $(20 \mathrm{~mL})$ was added to a mixture of cyclic iminium salt $\mathbf{4 e}$ $(1.81 \mathrm{~g}, 3.0 \mathrm{mmol})$ and KHMDS (0.60 g, $3.0 \mathrm{mmol})$ under stirring at room temperature, and the reaction mixture was stirred at room temperature for $20 \mathrm{~min}$. $\mathrm{CuCl}(0.45 \mathrm{~g}, 4.5 \mathrm{mmol})$ was added and the reaction mixture was stirred for another 2 hours. After the solvent was removed in vacuo, the crude product was extracted

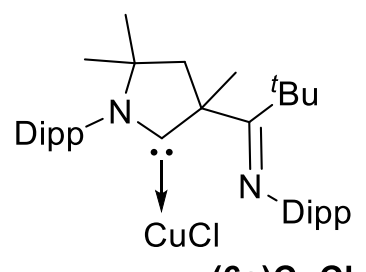

(6e) $\mathrm{CuCl}$ with $\mathrm{CH}_{2} \mathrm{Cl}_{2}(50 \mathrm{~mL})$. After $\mathrm{CH}_{2} \mathrm{Cl}_{2}$ was removed in vacuo, the solid was washed with 
hexane $(40 \mathrm{~mL})$ to afford a colorless powder $(1.37 \mathrm{~g}, 74 \%)$. m.p. $227-229{ }^{\circ} \mathrm{C}$ (dec.).

${ }^{1} \mathrm{H}$ NMR $\left(\mathrm{C}_{6} \mathrm{D}_{6}, 500 \mathrm{MHz}, \mathrm{ppm}\right):$ 7.12-7.08 (m, 2H, $\left.m-\mathrm{H}\right), 7.02-6.92$ (m, 4H, ArH), 3.60 (sept, $J=7.0 \mathrm{~Hz}, 1 \mathrm{H}, \mathrm{C} H \mathrm{Me}_{2}$ ), 3.03 (sept, $J=7.0 \mathrm{~Hz}, 1 \mathrm{H}, \mathrm{CHMe}$ ), 2.85 (sept, $J=7.0 \mathrm{~Hz}, 1 \mathrm{H}$, $\mathrm{CHMe}_{2}$ ), 2.79 (sept, $\left.J=7.0 \mathrm{~Hz}, 1 \mathrm{H}, \mathrm{C} H \mathrm{Me}_{2}\right), 2.26\left(\mathrm{~d}, J=13.5 \mathrm{~Hz}, 1 \mathrm{H}, \mathrm{CH}_{2}\right), 1.77$ (d, $J=$ $\left.13.5 \mathrm{~Hz}, 1 \mathrm{H}, \mathrm{CH}_{2}\right), 1.60$ (s, $\left.3 \mathrm{H}, \mathrm{CMe}\right), 1.60$ (d, $\left.J=7.0 \mathrm{~Hz}, 3 \mathrm{H}, \mathrm{CH} M e_{2}\right), 1.54$ (d, $J=7.0 \mathrm{~Hz}$, 3H, CHMe $), 1.47$ (d, J=7.0 Hz, 3H, CHMe $), 1.29$ (d, $J=7.0 \mathrm{~Hz}, 3 \mathrm{H}, \mathrm{CHMe}$ ), 1.18 (d, $J=$ $7.0 \mathrm{~Hz}, 3 \mathrm{H}, \mathrm{CHMe}$ ), 1.16 (d, J=7.0 Hz, 3H, CHMe $), 1.15$ (d, J=7.0 Hz, 3H, CHMe $), 1.06$ (s, 9H, CMe $), 1.02$ (d, $J=7.0 \mathrm{~Hz}, 3 \mathrm{H}, \mathrm{CHMe}), 0.92\left(\mathrm{~s}, 6 \mathrm{H}, \mathrm{CMe} e_{2}\right) \cdot{ }^{13} \mathrm{C}\left\{{ }^{1} \mathrm{H}\right\} \mathrm{NMR}\left(\mathrm{C}_{6} \mathrm{D}_{6}\right.$, $125 \mathrm{MHz}, \mathrm{ppm}$ ): $\delta=242.1$ (carbene $C$ ), 178.4 (imine $C$ ), 152.1, 146.4, 145.6, 145.4, 134.9, 134.5, 133.5, 130.1, 125.2, 124.9, 123.9, 123.1, $122.3(\mathrm{ArC}), 79.7\left(C_{\mathrm{q}}\right), 69.1\left(C_{\mathrm{q}}\right), 51.3\left(\mathrm{CH}_{2}\right)$, $44.1\left(C_{\mathrm{q}}\right), 29.9\left(\mathrm{CMe}_{3}\right), 29.3,29.2,29.1,28.7,28.6,28.3,27.4,27.3,27.1,26.1,25.1,23.6$, 22.9, 22.5, 22.2. HRMS: $\mathrm{m} / \mathrm{z}$ calculated for $\left[\mathrm{C}_{36} \mathrm{H}_{54} \mathrm{CuN}_{2}\right]^{+} \mathrm{M}+577.3578$, found 577.3582 .

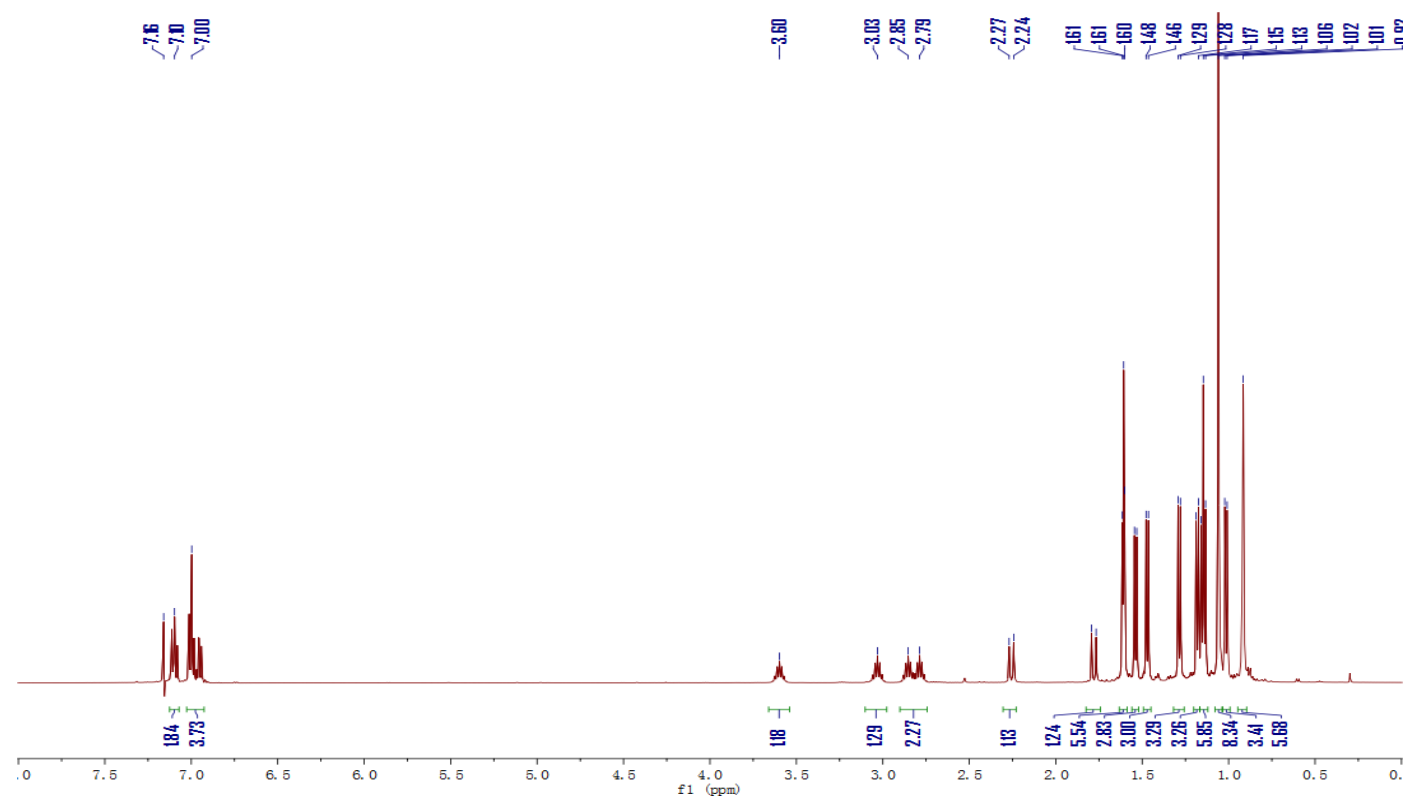

Figure S49. ${ }^{1} \mathrm{H}$ NMR of (6e)CuCl $\left(\mathrm{C}_{6} \mathrm{D}_{6}, 500 \mathrm{MHz}\right)$. 


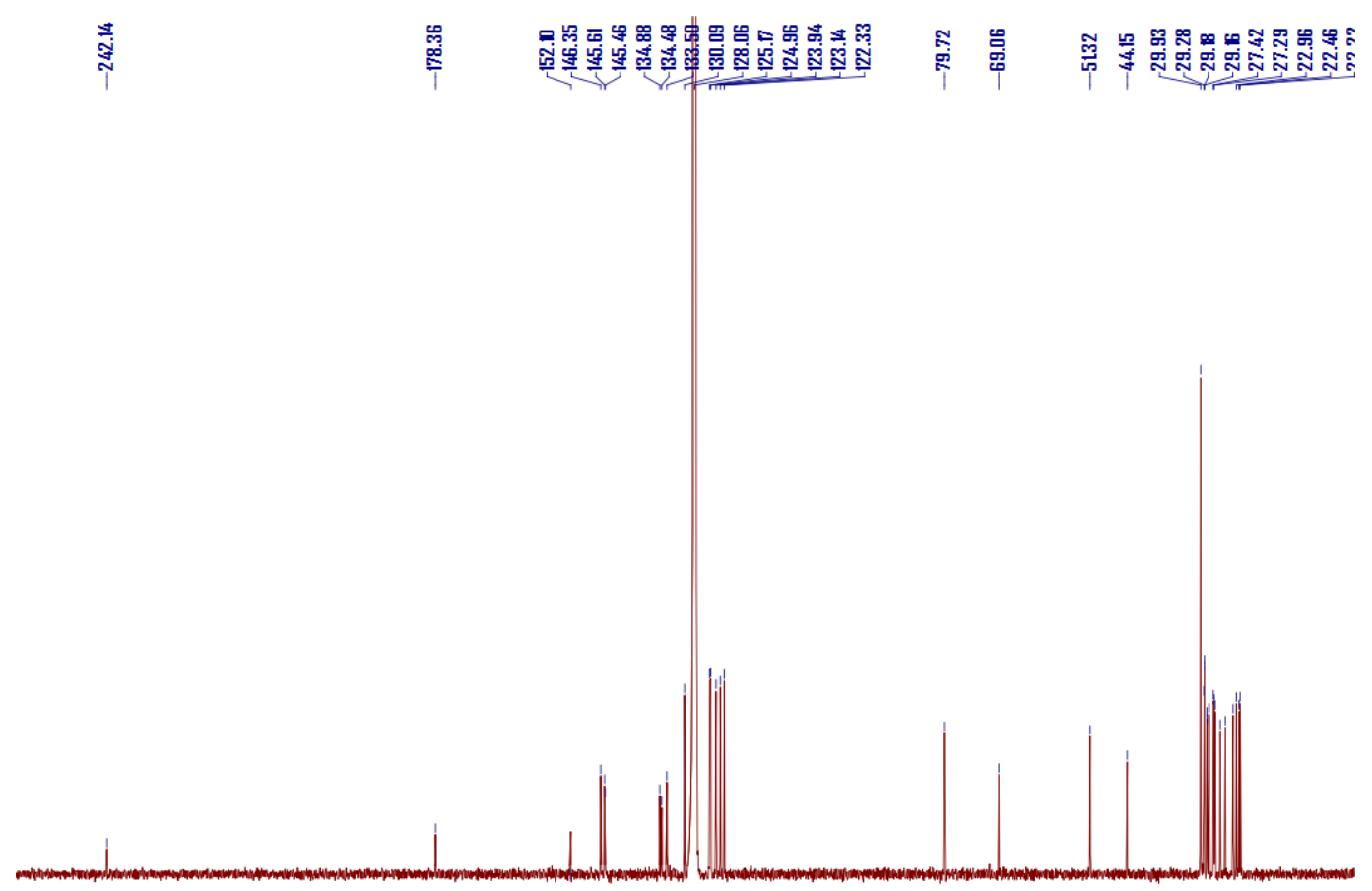

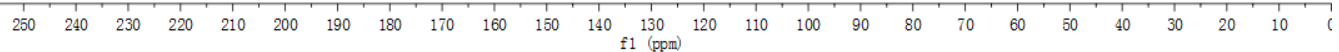

Figure S50. ${ }^{13} \mathrm{C}\left\{{ }^{1} \mathrm{H}\right\}$ NMR of (6e) CuCl $\left(\mathrm{C}_{6} \mathrm{D}_{6}, 125 \mathrm{MHz}\right)$.

\section{Copper trifluoromethanesulfonate complex (6e)CuOTf}

$\mathrm{CH}_{2} \mathrm{Cl}_{2}(20 \mathrm{~mL})$ was added to a mixture of copper complex (6e)CuCl (614 mg, $1.0 \mathrm{mmol})$ and AgOTf (257 mg, $1.0 \mathrm{mmol})$ under stiring at room temperature, and the reaction mixture was stirred at room temperature for 2 hours. The $\mathrm{CH}_{2} \mathrm{Cl}_{2}$ solution was filtered, volatiles were removed in vacuo, and the crude product was washed with hexane $(25 \mathrm{~mL})$ to afford a colorless powder

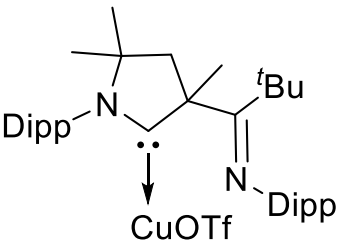

(6e)CuOTf (660 mg, 91\%). m.p. $171{ }^{\circ} \mathrm{C}(\mathrm{dec}$.$) .$

${ }^{1} \mathrm{H}$ NMR $\left(\mathrm{C}_{6} \mathrm{D}_{6}, 300 \mathrm{MHz}, \mathrm{ppm}\right): \delta=7.16-6.85(\mathrm{~m}, 6 \mathrm{H}, \mathrm{Ar} H), 3.42$ (sept, $J=6.9 \mathrm{~Hz}, 1 \mathrm{H}$, $\mathrm{C} H \mathrm{Me}_{2}$ ), 2.93 (sept, $J=6.9 \mathrm{~Hz}, 1 \mathrm{H}, \mathrm{C} H \mathrm{Me}_{2}$ ), 2.82-2.62 (m, 2H, CHMe $), 2.20$ (d, $J=12.6$ $\left.\mathrm{Hz}, 1 \mathrm{H}, \mathrm{CH}_{2}\right), 1.79$ (d, $\left.J=12.6 \mathrm{~Hz}, 1 \mathrm{H}, \mathrm{CH}_{2}\right), 1.66(\mathrm{~s}, 3 \mathrm{H}, \mathrm{CMe}), 1.50-1.40(\mathrm{~m}, 9 \mathrm{H}, \mathrm{CMe}$ and $\mathrm{CHMe}$ ), 1.23 (d, $J=6.9 \mathrm{~Hz}, 3 \mathrm{H}, \mathrm{CHMe}$ ), $1.13(\mathrm{~m}, 6 \mathrm{H}, \mathrm{CMe}$ and $\mathrm{CHMe}$ ), 1.05-0.80 (m, $21 \mathrm{H}, \mathrm{CMe}_{3}$ and $\left.\mathrm{CHMe} 2\right) .{ }^{13} \mathrm{C}\left\{{ }^{1} \mathrm{H}\right\}$ NMR $\left(\mathrm{C}_{6} \mathrm{D}_{6}, 125 \mathrm{MHz}, \mathrm{ppm}\right): \delta=239.9$ (carbene $C$ ), 179.7 (imine $C$ ), 146.1, 145.5, 145.1, 135.0, 134.3, 133.6, 130.4, 125.4, 125.1, 124.1, 123.5, $122.5(\mathrm{ArC}), 80.6\left(C_{\mathrm{q}}\right), 69.1\left(C_{\mathrm{q}}\right), 51.3\left(\mathrm{CH}_{2}\right), 44.2\left(C_{\mathrm{q}}\right), 29.8\left(\mathrm{CMe}_{3}\right), 29.3,29.2,28.8,28.6$, 28.2, 27.4, 27.3, 26.4, 26.0, 25.1, 23.5, 22.8, 22.7, 22.1. HRMS: $\mathrm{m} / \mathrm{z}$ calculated for $\left[\mathrm{C}_{36} \mathrm{H}_{54}\right.$ $\left.\mathrm{Cu} \mathrm{N}_{2}\right]^{+} 577.3578$ found 577.3574 . 


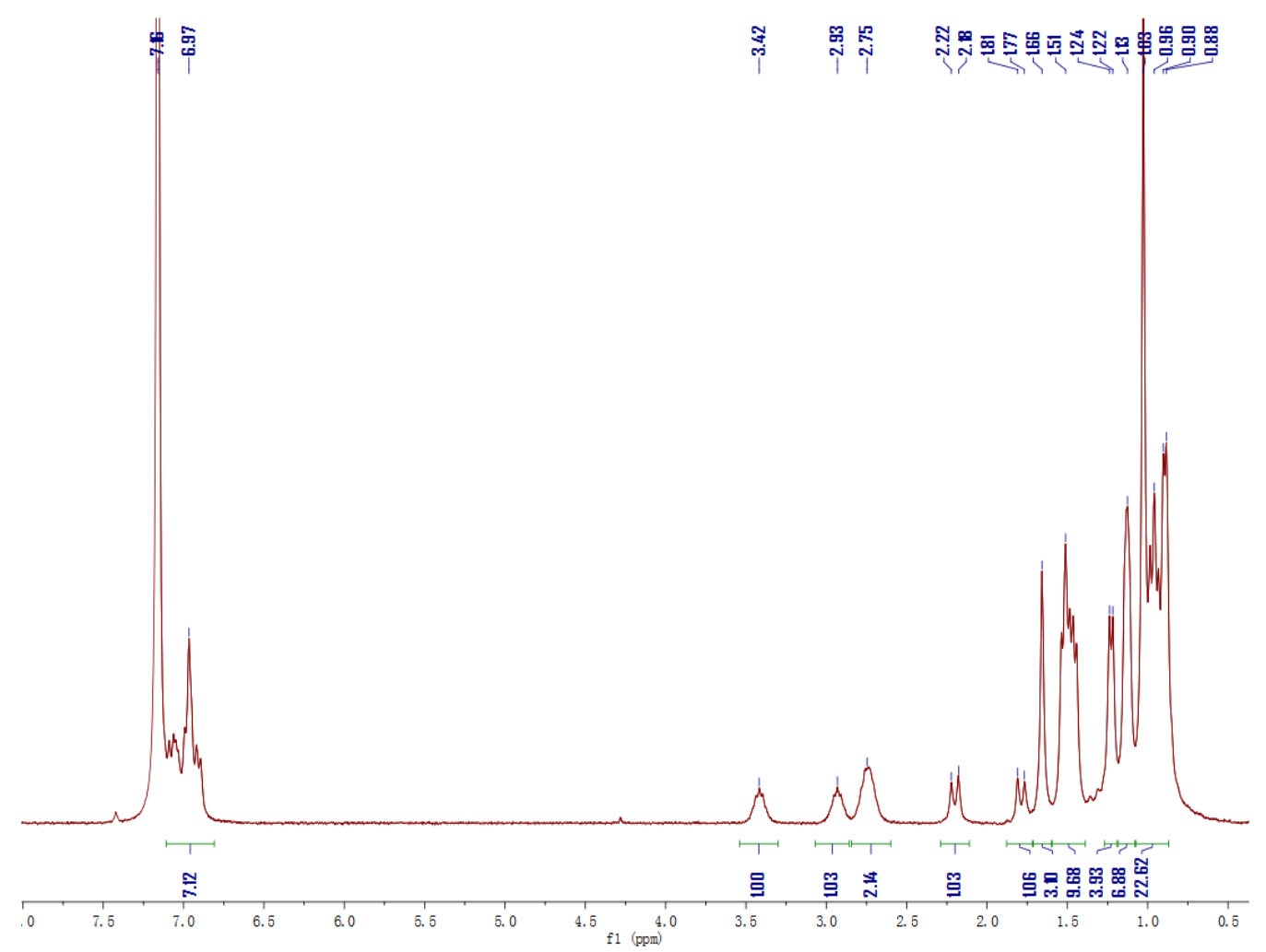

Figure S51. ${ }^{1} \mathrm{H}$ NMR of (6e)CuOTf $\left(\mathrm{C}_{6} \mathrm{D}_{6}, 300 \mathrm{MHz}\right)$.

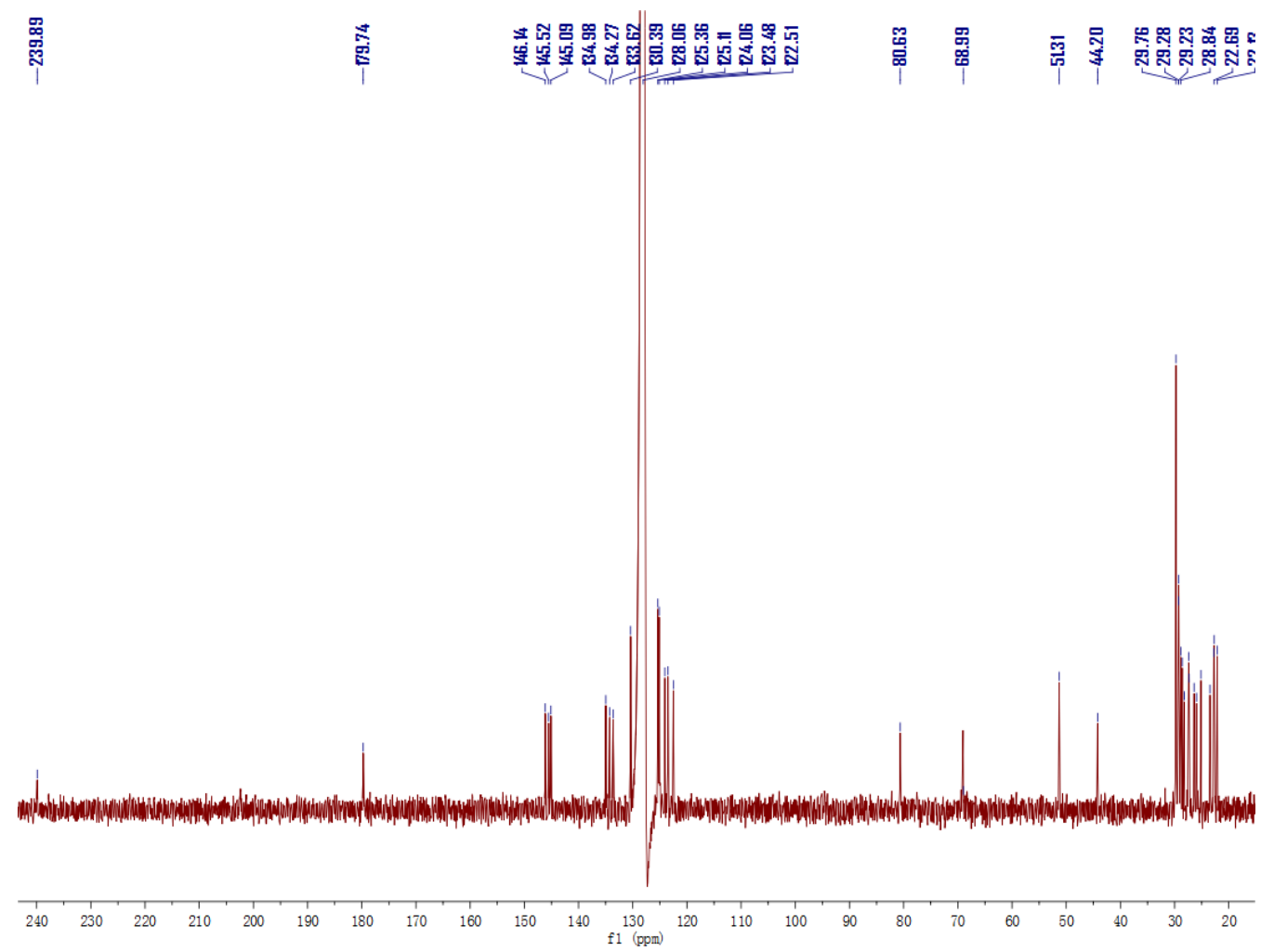

Figure S52. ${ }^{13} \mathrm{C}\left\{{ }^{1} \mathrm{H}\right\}$ NMR of (6e)CuOTf $\left(\mathrm{C}_{6} \mathrm{D}_{6}, 125 \mathrm{MHz}\right)$. 


\section{Gold chloride complex (6e)AuCl}

THF $(20 \mathrm{~mL})$ was added to a mixture of cyclic iminium salt $4 \mathbf{e}$ (301 $\mathrm{mg}, 0.5 \mathrm{mmol}$ ) and KHMDS (100 $\mathrm{mg}, 0.5 \mathrm{mmol})$ under stirring at room temperature, and the reaction mixture was stirred at room temperature for $15 \mathrm{~min}$. AuCl(THT) (160 mg, $0.5 \mathrm{mmol}$ ) was added and the reaction mixture was stirred for another 12 hours.<smiles>CC1(C)CC(C)(C(C)(C)C)C(=NOc2ccccc2)N1[Pb]</smiles>

(6e)AuCl The THF solution was filtered to another Schlenk flask and the solvent was removed in vacuo. The solid was washed with hexane $(20 \mathrm{~mL})$ to afford a colorless powder $(295 \mathrm{mg}, 79 \%)$. m.p. $277-279^{\circ} \mathrm{C}$ (dec.).

${ }^{1} \mathrm{H}$ NMR $\left(\mathrm{C}_{6} \mathrm{D}_{6}, 500 \mathrm{MHz}, \mathrm{ppm}\right)$ : 7.13-7.06 (m, 2H, $\left.m-\mathrm{H}\right), 7.02-6.92(\mathrm{~m}, 4 \mathrm{H}, \mathrm{Ar} H), 3.86$ (sept, $J=7.0 \mathrm{~Hz}, 1 \mathrm{H}, \mathrm{C} H \mathrm{Me}_{2}$ ), 3.03 (sept, $J=7.0 \mathrm{~Hz}, 1 \mathrm{H}, \mathrm{C} H \mathrm{Me}_{2}$ ), 2.95 (sept, $J=7.0 \mathrm{~Hz}, 1 \mathrm{H}$, CHMe $\left.\mathrm{M}_{2}\right), 2.75$ (sept, $\left.J=7.0 \mathrm{~Hz}, 1 \mathrm{H}, \mathrm{C} H \mathrm{Me}_{2}\right), 2.32$ (d, $J=13.5 \mathrm{~Hz}, 1 \mathrm{H}, \mathrm{CH}$ ), $1.80(\mathrm{~d}, J=$ $13.5 \mathrm{~Hz}, 1 \mathrm{H}, \mathrm{CH}_{2}$ ), 1.70 (s, 3H, CMe), 1.66 (d, $J=7.0 \mathrm{~Hz}, 3 \mathrm{H}, \mathrm{CHMe}$ ), 1.54 (d, $J=7.0 \mathrm{~Hz}$, $3 \mathrm{H}, \mathrm{CHMe}$ ), 1.46 (d, J=7.0 Hz, 3H, CHMe $), 1.30$ (d, $J=7.0 \mathrm{~Hz}, 3 \mathrm{H}, \mathrm{CHMe}$ ), 1.22 (d, $J=$ $7.0 \mathrm{~Hz}, 3 \mathrm{H}, \mathrm{CHMe}$ ), 1.21 (d, J=7.0 Hz, 3H, CHMe $), 1.13$ (d, J=7.0 Hz, 3H, CHMe 2 ), 1.05 (s, 9H, CMe 3 ), 1.01 (d, $J=7.0 \mathrm{~Hz}, 3 \mathrm{H}, \mathrm{CHMe}$ ) 0.92 (s, 6H, CMe 2$).{ }^{13} \mathrm{C}\left\{{ }^{1} \mathrm{H}\right\}$ NMR $\left(\mathrm{C}_{6} \mathrm{D}_{6}\right.$, $125 \mathrm{MHz}, \mathrm{ppm}$ ): $\delta=231.7$ (carbene $C$ ), 177.4 (imine $C$ ), 146.3, 145.6, 145.5, 135.1, 134.1, 133.7, 130.2, 125.3, 125.1, 124.0, 123.3, $122.3(\mathrm{ArC}), 79.2\left(C_{\mathrm{q}}\right), 69.4\left(C_{\mathrm{q}}\right), 51.8\left(\mathrm{CH}_{2}\right), 43.9$ $\left(C_{\mathrm{q}}\right), 29.8\left(\mathrm{CMe}_{3}\right), 29.3,29.2,28.9,28.8,28.7,28.6,28.4,27.9,27.3,27.2,26.4,25.1,23.4$, 23.2, 22.5. HRMS: $\mathrm{m} / \mathrm{z}$ calculated for $\left[\mathrm{C}_{36} \mathrm{H}_{54} \mathrm{AuN}_{2}\right]^{+} \mathrm{M}+711.3947$, found 711.3941 .

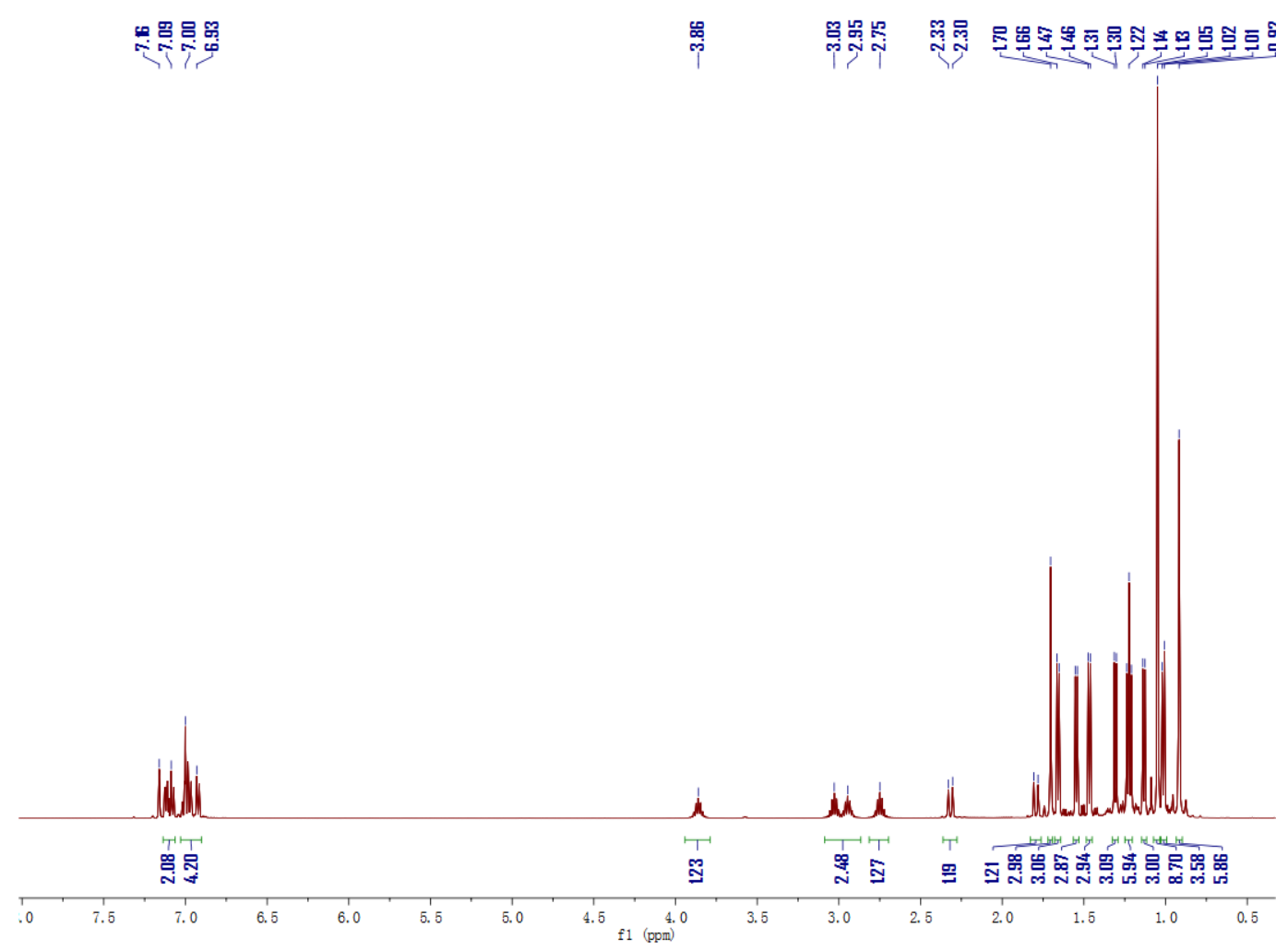

Figure S53. ${ }^{1} \mathrm{H}$ NMR of (6e) AuCl $\left(\mathrm{C}_{6} \mathrm{D}_{6}, 500 \mathrm{MHz}\right)$. 

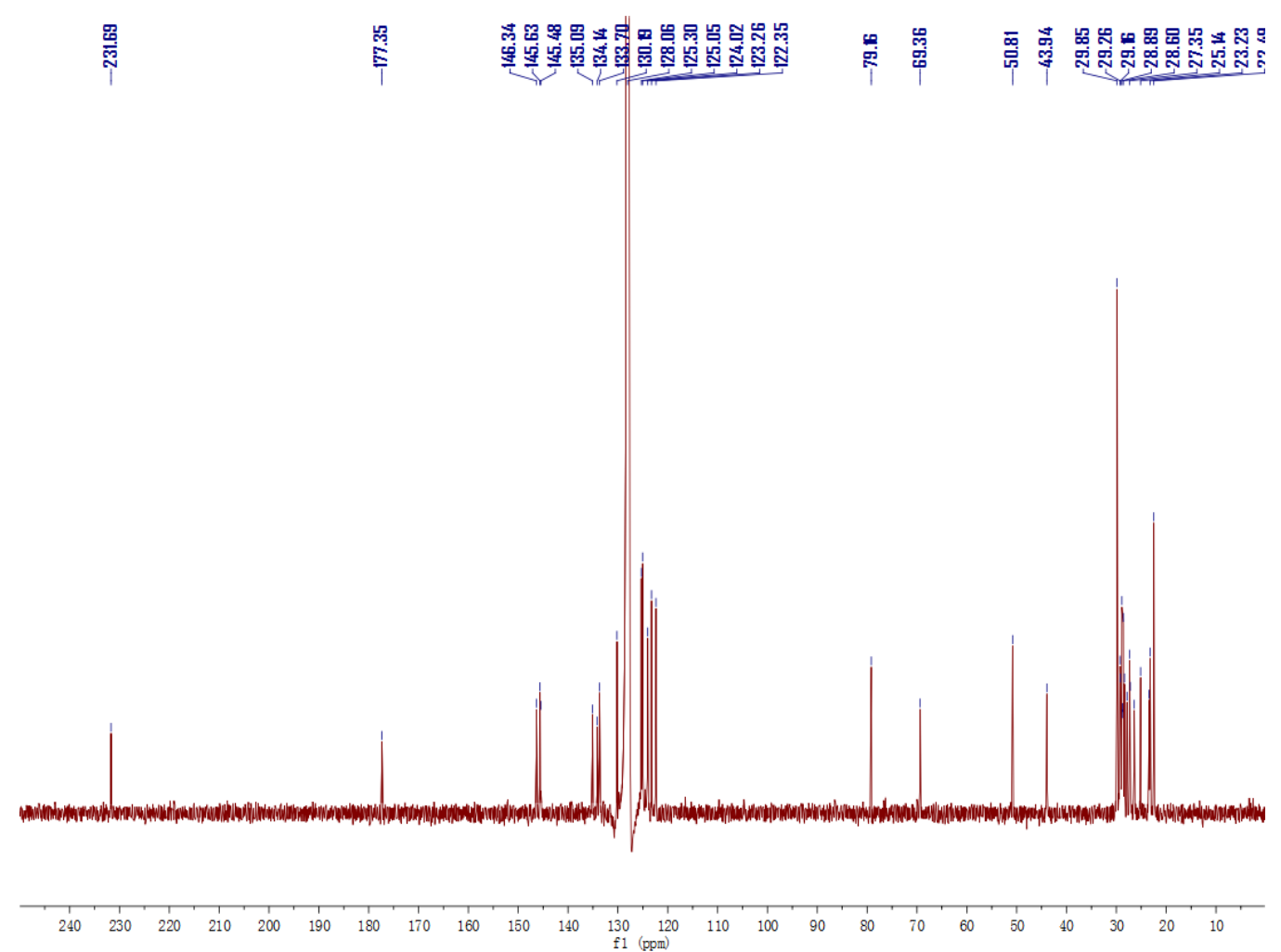

Figure S54. ${ }^{13} \mathrm{C}\left\{{ }^{1} \mathrm{H}\right\}$ NMR of (6e) AuCl $\left(\mathrm{C}_{6} \mathrm{D}_{6}, 125 \mathrm{MHz}\right)$.

\section{Copper chloride complex (6f)CuCl}

Cyclic iminium salt $4 \mathbf{f}(1.5 \mathrm{~g}, 2.78 \mathrm{mmol})$ was put in a Schlenk flask together with 1 eq of KHMDS $(528 \mathrm{mg})$. THF $(20 \mathrm{~mL})$ was added and it was stirred for $5 \mathrm{~min}$, during which a colorless precipitate formed. The solution was transferred via canula to another Schlenk flask, which contained 1.1 eq. of $\mathrm{CuCl}$ (334 mg). It was stirred over night at room temperature. The solvent was

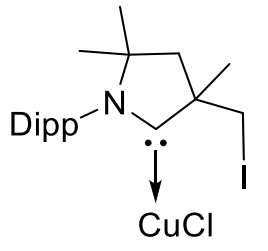

(6f) $\mathrm{CuCl}$ evaporated in vacuo, it was extracted with benzene, and the extract was washed with hexanes after evaporation, until the solution was colorless. The residue was washed with $1 \mathrm{~mL}$ of benzene at $5{ }^{\circ} \mathrm{C}$ and dried. A colorless powder was obtained $(1.06 \mathrm{~g}, 75 \%)$. m.p. $199{ }^{\circ} \mathrm{C}$ (dec).

${ }^{1} \mathrm{H}$ NMR $\left(\mathrm{CDCl}_{3}, 400 \mathrm{MHz}, \mathrm{ppm}\right): \delta=7.13(\mathrm{~m}, 1 \mathrm{H}, \mathrm{Ar} H), 6.99(\mathrm{~m}, 2 \mathrm{H}, \operatorname{Ar} H), 3.32(\mathrm{~d}, J=$ $\left.10.1 \mathrm{~Hz}, 1 \mathrm{H}, \mathrm{CH}_{2}\right), 2.99(\mathrm{~m}, 1 \mathrm{H}), 2.80(\mathrm{~d}, J=10.1 \mathrm{~Hz}, 1 \mathrm{H}), 2.58(\mathrm{~m}, 1 \mathrm{H}), 1.81(\mathrm{~d}, J=13.5$ $\left.\mathrm{Hz}, 1 \mathrm{H}, \mathrm{CH}_{2}\right), 1.41$ (s, 3H, Me), 1.39 (s, 3H, Me), 1.25 (d, $\left.J=13.5 \mathrm{~Hz}, 1 \mathrm{H}, \mathrm{CH}_{2}\right), 1.10$ (d, $J=$ $6.7 \mathrm{~Hz}, 3 \mathrm{H}, M e), 1.04(\mathrm{~m}, 6 \mathrm{H}, M e), 0.82(\mathrm{~s}, 3 \mathrm{H}, M e), 0.77(\mathrm{~s}, 3 \mathrm{H}, M e) .{ }^{13} \mathrm{C}\left\{{ }^{1} \mathrm{H}\right\} \mathrm{NMR}$ $\left(\mathrm{CDCl}_{3}, 125 \mathrm{MHz}, \mathrm{ppm}\right): \delta=244.8$ (carbene), 145.3, 144.8, 135.6, 130.0, 124.9, $124.8(\mathrm{ArC})$, $81.9\left(C_{\mathrm{q}}\right), 57.9\left(\mathrm{CH}_{2}\right), 46.9\left(\mathrm{CH}_{2}\right), 29.3,29.0,28.8,28.6,27.6,27.6,27.3,22.5,22.0,17.4$ $\left(\mathrm{CH}_{2}\right)$. HRMS calculated for $\left[\mathrm{C}_{20} \mathrm{H}_{30} \mathrm{CuIN}\right]^{+} 474.0719$, found 474.0713 . 


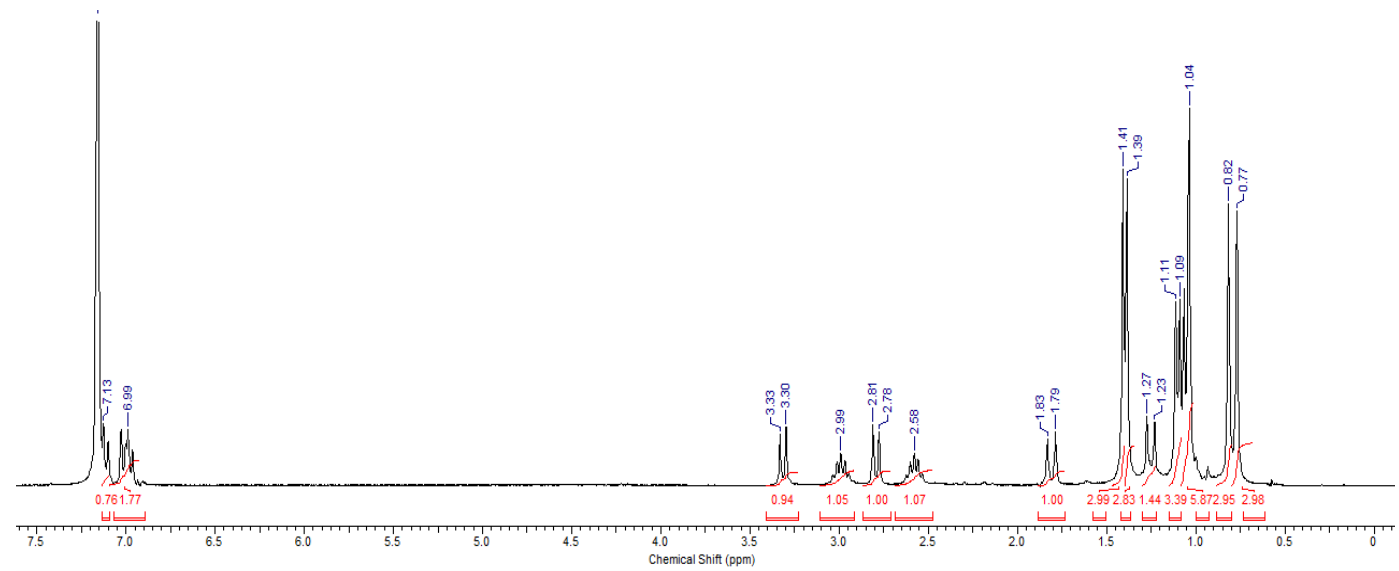

Figure S55. ${ }^{1} \mathrm{H} \mathrm{NMR}$ of (6f) CuCl $\left(\mathrm{CDCl}_{3}, 400 \mathrm{MHz}\right)$.

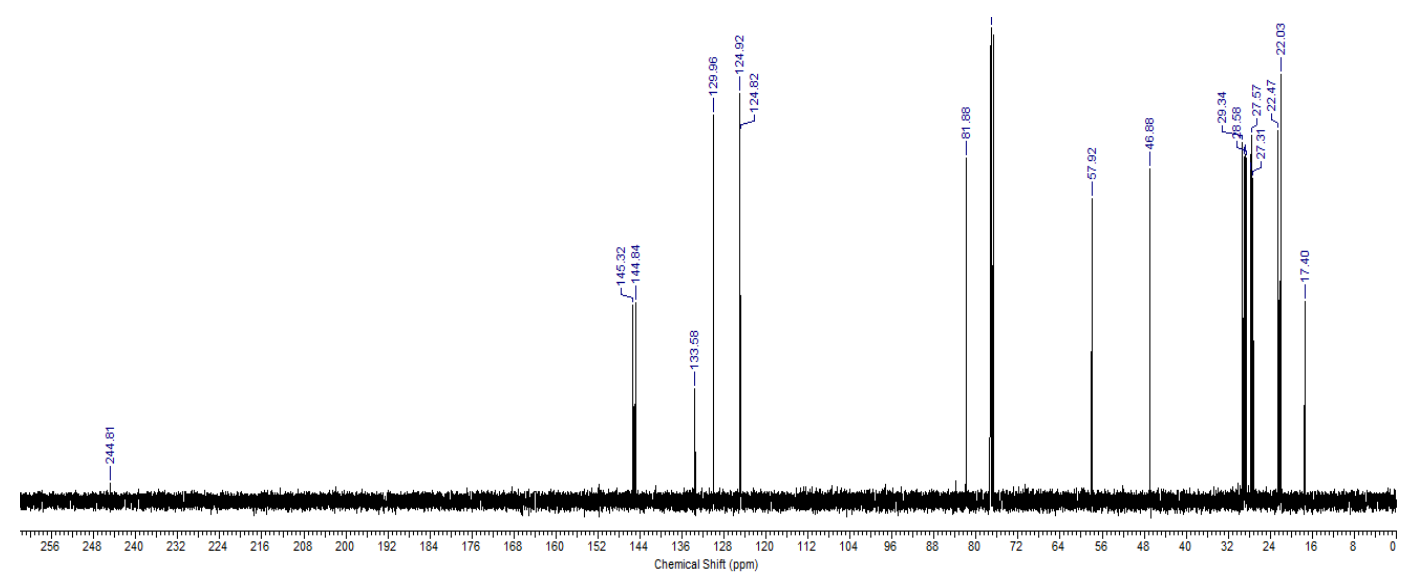

Figure S56. ${ }^{13} \mathrm{C}\left\{{ }^{1} \mathrm{H}\right\}$ NMR of (6f)CuCl $\left(\mathrm{CDCl}_{3}, 125 \mathrm{MHz}\right)$.

\section{Methylene diphenylphospino functionalized copper complex $(\mathbf{6 g}) \mathbf{C u C l}$}

THF (20 $\mathrm{mL})$ was added to a mixture of cyclic iminium salt $\mathbf{6 g}$ (1.20 g, $2 \mathrm{mmol})$ and KHMDS (0.399 g, $2 \mathrm{mmol})$ under stirring at $0{ }^{\circ} \mathrm{C}$. The reaction mixture was stirred at room temperature for 30 min. The THF solution was filtered to another Schlenk flask and $\mathrm{CuCl}$ (208 mg, $2.1 \mathrm{mmol}$ ) was added. The reaction mixture was stirred at room temperature for 12 hours. After THF was removed

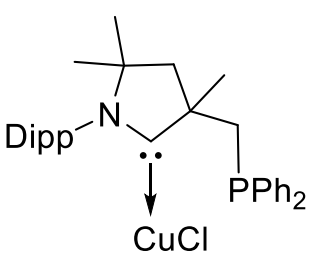

$(6 \mathrm{~g}) \mathrm{CuCl}$ in vacuo, the solid was extracted with benzene $(30 \mathrm{~mL})$. After benzene was removed in vacuo, the solid was washed with cold ether $(50 \mathrm{~mL})$ to afford an orange powder $(0.66 \mathrm{~g}, 58 \%)$. m.p. $154{ }^{\circ} \mathrm{C}(\mathrm{dec})$.

${ }^{1} \mathrm{H}$ NMR $\left(\mathrm{C}_{6} \mathrm{D}_{6}, 300 \mathrm{MHz}, \mathrm{ppm}\right): \delta=7.70-7.55$ (br, 3H, $\left.\mathrm{ArH}\right), 7.10-6.90$ (br, 10H, $\mathrm{ArH}$ ), 3.10-2.90 (m, 2H, CHMe 2 and $\left.\mathrm{CH}_{2}\right), 2.85-2.65\left(\mathrm{~m}, 2 \mathrm{H}, \mathrm{CH}_{2}\right), 2.45-2.30\left(\mathrm{br}, 1 \mathrm{H}, \mathrm{CHMe}_{2}\right.$ ), $1.75\left(\mathrm{~d}, J=13.2 \mathrm{~Hz}, 1 \mathrm{H}, \mathrm{CH}_{2}\right), 1.53$ (d, $J=6.6 \mathrm{~Hz}, 3 \mathrm{H}, \mathrm{CHMe}$ ), 1.42 (d, $J=6.6 \mathrm{~Hz}, 3 \mathrm{H}$, $\mathrm{CHMe}$ ), 1.19 (br, 3H, CMe), 1.14 (d, $J=6.6 \mathrm{~Hz}, 3 \mathrm{H}, \mathrm{CHMe}$ ), 1.02 (d, $J=6.6 \mathrm{~Hz}, 3 \mathrm{H}$, $\mathrm{CHMe}$ ), 0.94 (br, 3H, CMe), 0.81 (br, 3H, CMe). ${ }^{13} \mathrm{C}\left\{{ }^{1} \mathrm{H}\right\} \mathrm{NMR}\left(\mathrm{C}_{6} \mathrm{D}_{6}, 125 \mathrm{MHz}, \mathrm{ppm}\right): \delta=$ 248.4 (br, carbene $C$ ), 146.0, 145.5, 136.2 (d, $J=51.9 \mathrm{~Hz}), 134.7,133.8,129.7$ (d, $J=11.7$ $\mathrm{Hz}), 129.5,128.9,124.8(\mathrm{~d}, J=10.6 \mathrm{~Hz}),(\operatorname{ArC}), 80.9\left(\mathrm{C}_{\mathrm{q}}\right), 57.6,48.8,41.9,29.5,29.0,28.9$, 
28.4, 27.6, 22.6. ${ }^{31} \mathrm{P}\left\{{ }^{1} \mathrm{H}\right\}$ NMR $\left(\mathrm{C}_{6} \mathrm{D}_{6}, 121 \mathrm{MHz}, \mathrm{ppm}\right)$ : -19.8. HRMS: $\mathrm{m} / \mathrm{z}$ calculated for $\left[\mathrm{C}_{32} \mathrm{H}_{30} \mathrm{CuNP}\right]^{+}$532.2194, found 470.2972; iminium salt, copper complex not stable in ion source.

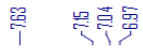

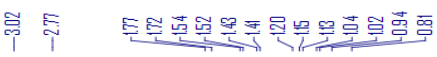

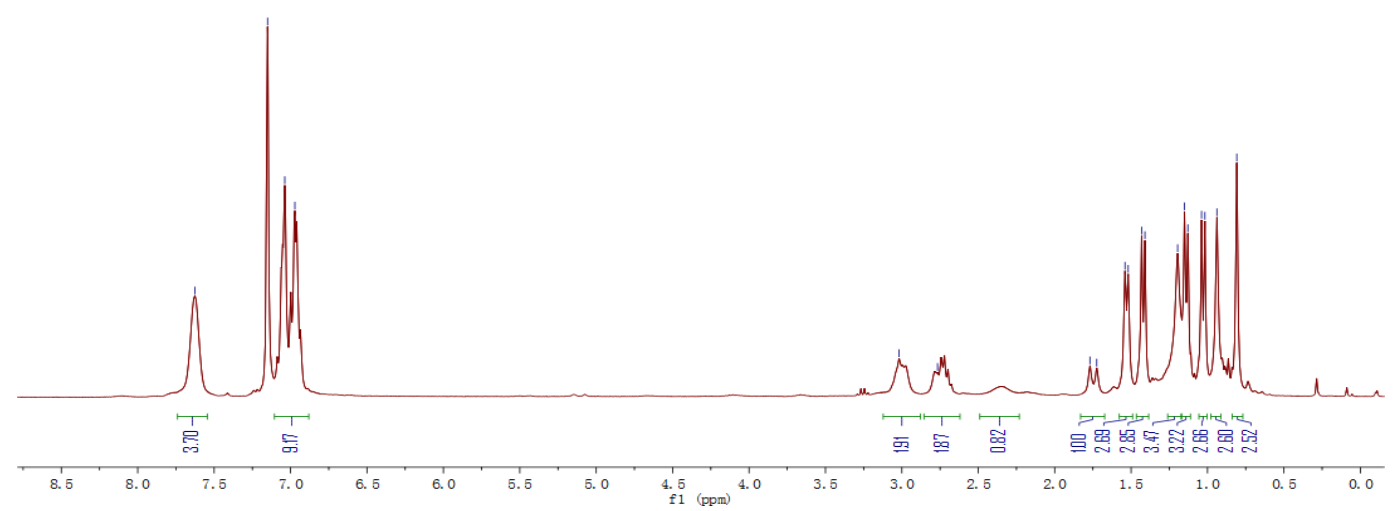

Figure S57. ${ }^{1} \mathrm{H}$ NMR of (6g) CuCl $\left(\mathrm{C}_{6} \mathrm{D}_{6}, 300 \mathrm{MHz}\right)$.

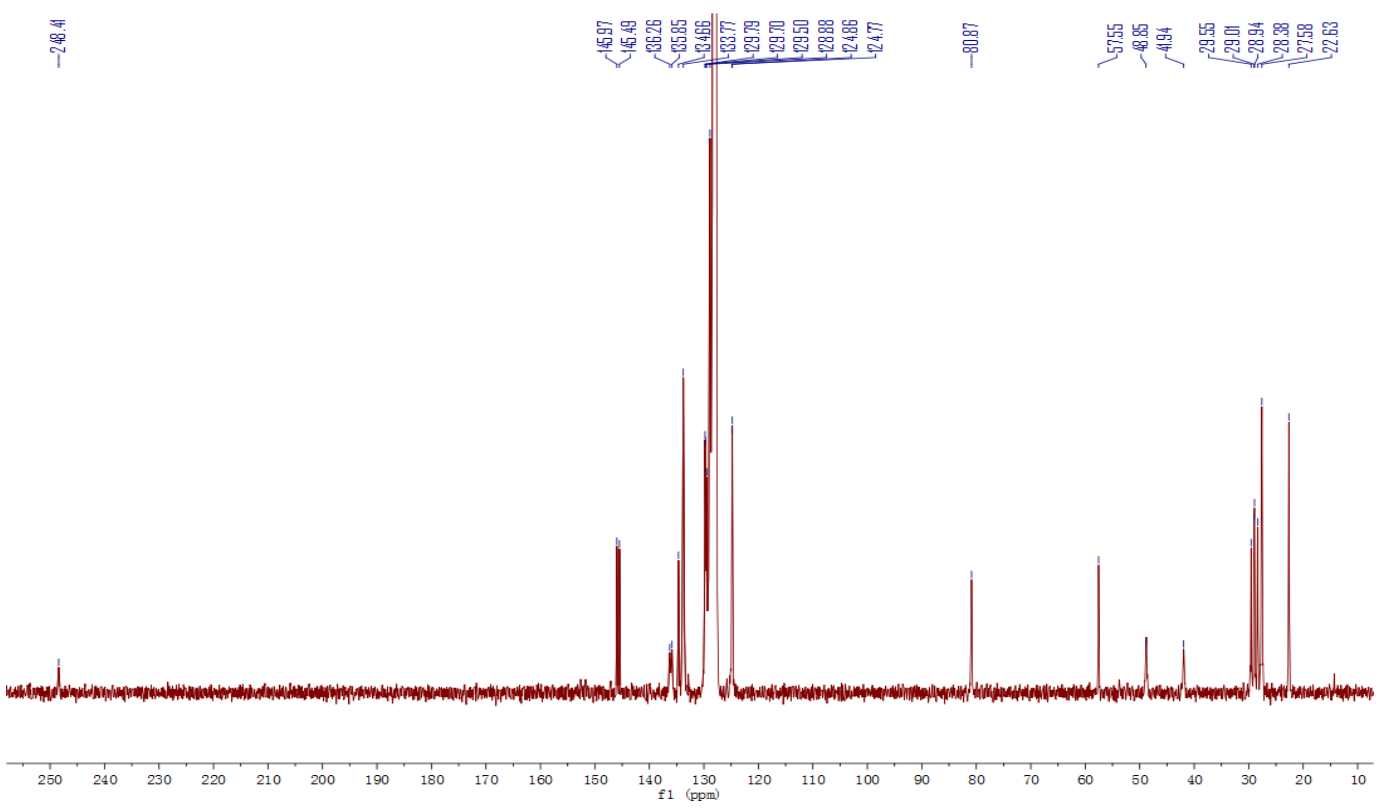

Figure S58. ${ }^{13} \mathrm{C}\left\{{ }^{1} \mathrm{H}\right\}$ NMR of (6g) $\mathbf{C u C l}\left(\mathrm{C}_{6} \mathrm{D}_{6}, 125 \mathrm{MHz}\right)$. 


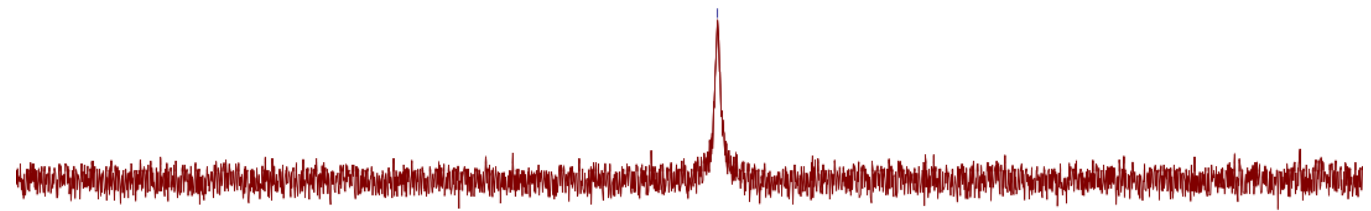

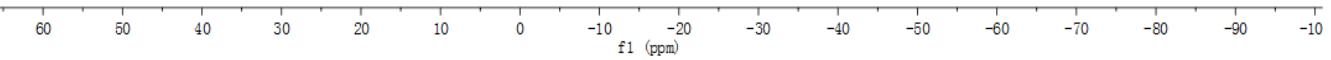

Figure 559. ${ }^{31} \mathrm{P}\left\{{ }^{1} \mathrm{H}\right\}$ NMR of $(\mathbf{6 g}) \mathbf{C u C l}\left(\mathrm{C}_{6} \mathrm{D}_{6}, 121 \mathrm{MHz}\right)$.

\section{Gold(III) biphenylene complex 7}

$\mathrm{CH}_{2} \mathrm{Cl}_{2}(1 \mathrm{~mL})$ was added to a mixture of (6e) $\mathbf{A u C l}(15 \mathrm{mg}$, $0.02 \mathrm{mmol}), \mathrm{K}\left(\mathrm{C}_{6} \mathrm{~F}_{5}\right)_{4}(14 \mathrm{mg}, 0.2 \mathrm{mmol})$ and biphenylene (15.2 $\mathrm{mg}, 0.1 \mathrm{mmol}$ ) at room temperature. The $\mathrm{CH}_{2} \mathrm{Cl}_{2}$ solution was filtered after $12 \mathrm{~h}$, and the solvent was removed in vacuo. The crude product was extracted with benzene (10 $\mathrm{mL}$ ) and volatiles were removed in vacuo. A colorless

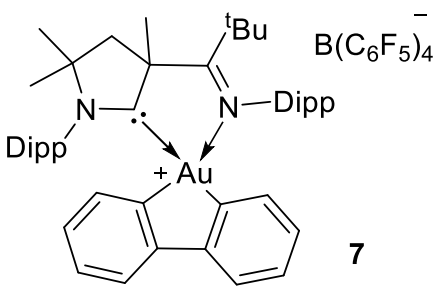
powder $(17 \mathrm{mg}, 55 \%)$ was obtained after it was washed with hexane $(10 \mathrm{~mL})$. m.p. $128-130$ (dec.).

${ }^{1} \mathrm{H}$ NMR $\left(\mathrm{C}_{6} \mathrm{D}_{6}, 300 \mathrm{MHz}, \mathrm{ppm}\right): 7.10-6.65(\mathrm{~m}, 10 \mathrm{H}, \mathrm{Ar} H), 6.49$ (t, $\left.J=7.5 \mathrm{~Hz}, 1 \mathrm{H}, \mathrm{Ar} H\right)$, 6.05-5.95 (m, 2H, $\operatorname{Ar} H), 4.79(\mathrm{~d}, J=8.1 \mathrm{~Hz}, 1 \mathrm{H}), 3.12(\mathrm{~d}, J=14.7 \mathrm{~Hz}, 1 \mathrm{H}, \mathrm{CH}$ ), 2.92 (sept, $J=6.9 \mathrm{~Hz}, 1 \mathrm{H}, \mathrm{C} H \mathrm{Me}_{2}$ ), 2.76 (sept, $J=6.9 \mathrm{~Hz}, 1 \mathrm{H}, \mathrm{C} H \mathrm{Me}_{2}$ ), 2.61 (sept, $J=6.9 \mathrm{~Hz}, 1 \mathrm{H}$, $\mathrm{C} H \mathrm{Me}_{2}$ ), 2.41 (sept, $J=6.9 \mathrm{~Hz}, 1 \mathrm{H}, \mathrm{C} H \mathrm{Me}_{2}$ ), 1.87 (d, $\left.J=14.7 \mathrm{~Hz}, 1 \mathrm{H}, \mathrm{C} H_{2}\right), 1.57$ (s, $3 \mathrm{H}$, $\mathrm{CMe}$ ), 1.33-1.13 (m, 12H, CMe and $\mathrm{CHMe}_{2}$ ), 1.15-1.08 (m, 6H, CMe and $\mathrm{CHMe}$ ), 1.04-0.86 (m, 18H, $\mathrm{CMe}_{3}$ and $\mathrm{CHMe}$ ), $0.56\left(\mathrm{~d}, J=6.9 \mathrm{~Hz}, 3 \mathrm{H}, \mathrm{CHMe} e_{2}\right){ }^{13} \mathrm{C}\left\{{ }^{1} \mathrm{H}\right\} \mathrm{NMR}\left(\mathrm{C}_{6} \mathrm{D}_{6}, 125\right.$ MHz, ppm): $\delta=251.0$ (carbene $C$ ), 196.2 (imine $C$ ), 162.3, 152.7, 150.1, 148.3, 145.3, 143.5, $142.7,140.5,140.0,139.0,138.0,136.2$, 135.7, 132.7, 131.6, 129.9, 129.5, 125.9, 125.8, 125.6, 123.9, 122.5, $121.5(\operatorname{ArC}), 87.7\left(C_{\mathrm{q}}\right), 78.5,44.8,44.1,33.1,32.4,30.9,30.1,29.5,29.0$, 28.9, 25.8, 25.3, 24.8, 24.7, 24.2, 23.7, 22.6, 21.7. HRMS: $\mathrm{m} / \mathrm{z}$ calculated for $\left[\mathrm{C}_{48} \mathrm{H}_{62} \mathrm{AuN}_{2}\right]^{+}$ 863.4573, found 863.4568. 


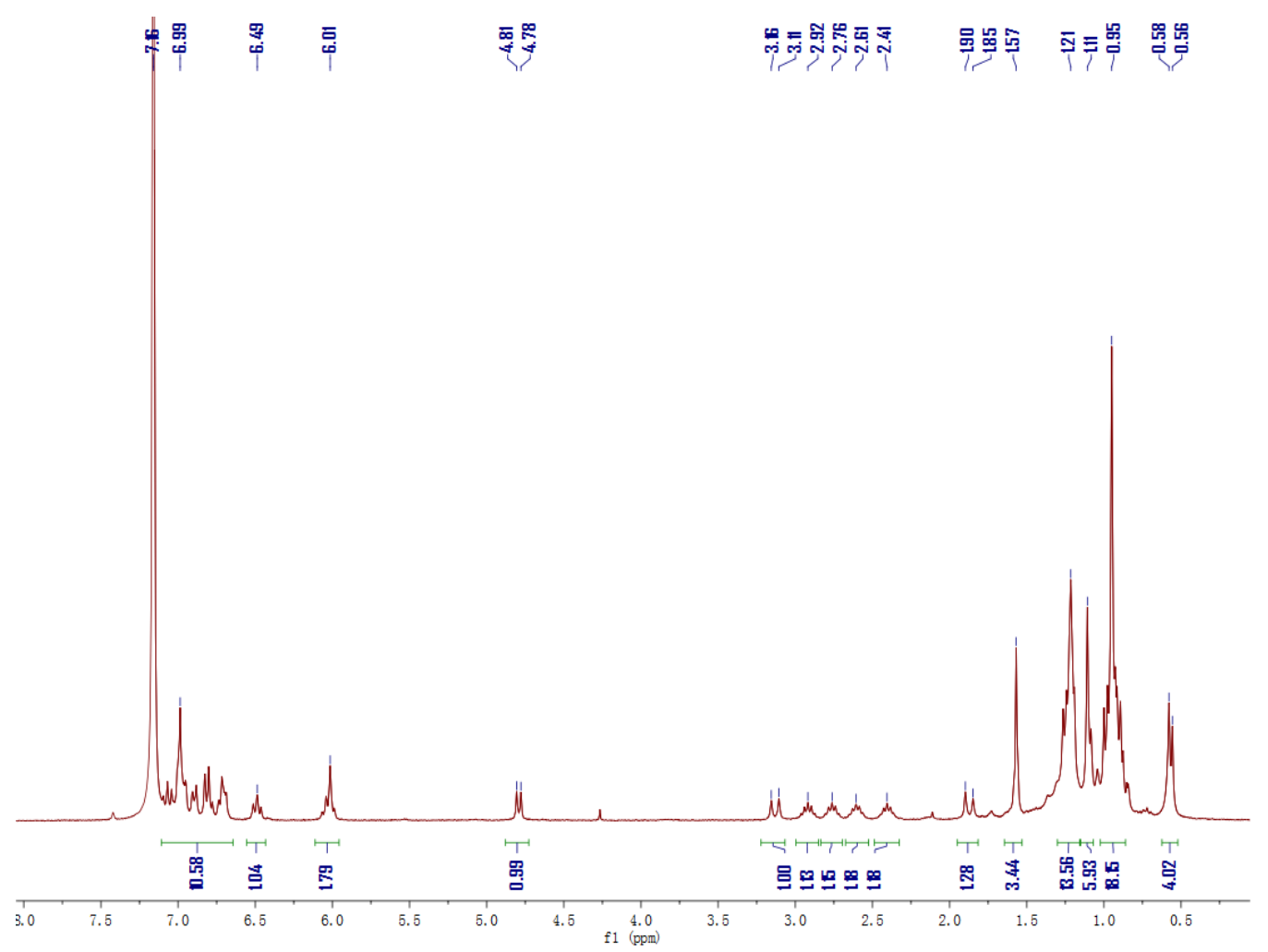

Figure S60. ${ }^{1} \mathrm{H}$ NMR of $7\left(\mathrm{C}_{6} \mathrm{D}_{6}, 300 \mathrm{MHz}\right)$.

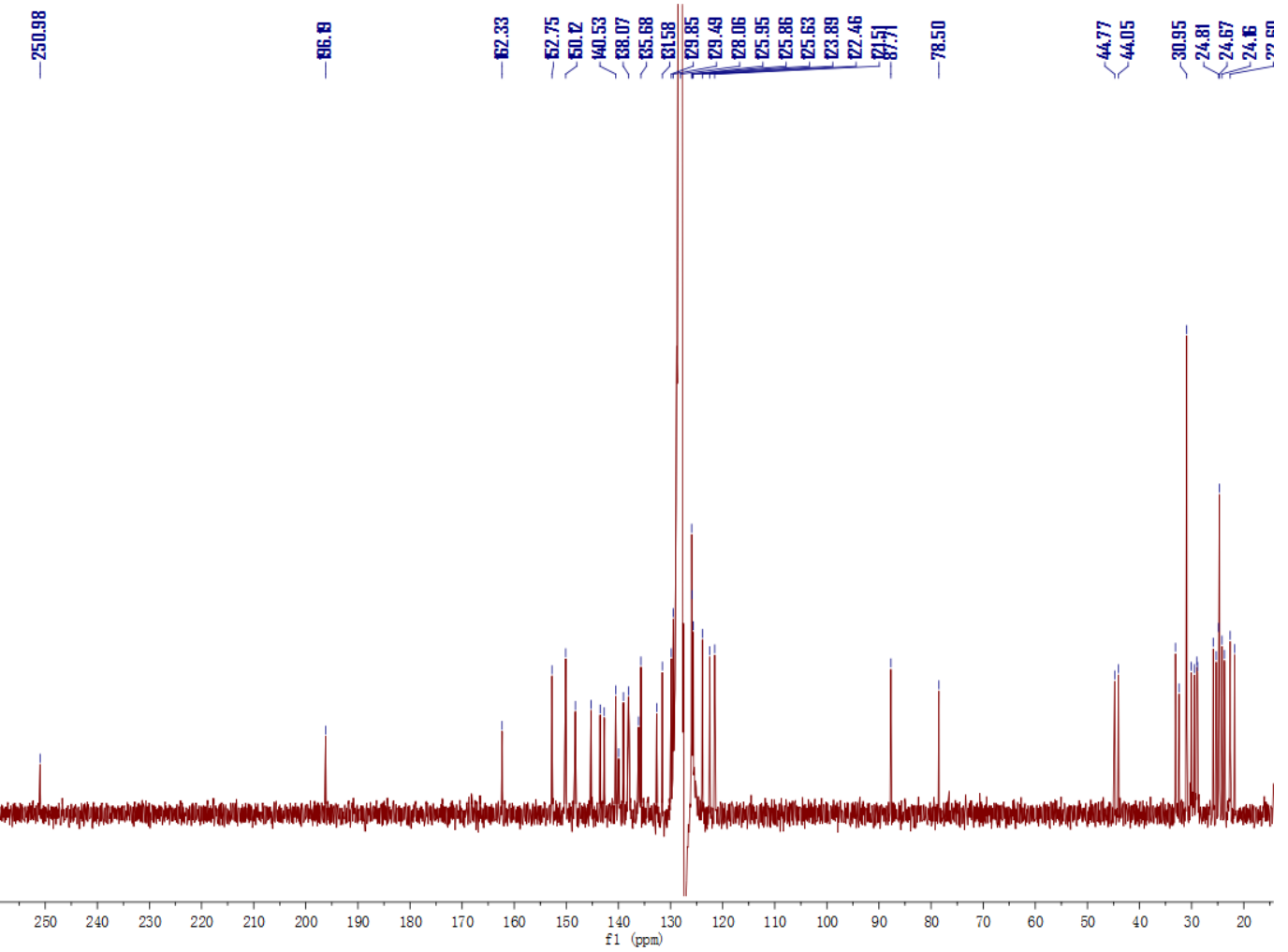

Figure S61. ${ }^{13} \mathrm{C}\left\{{ }^{1} \mathrm{H}\right\}$ NMR of $7\left(\mathrm{C}_{6} \mathrm{D}_{6}, 125 \mathrm{MHz}\right)$. 


\section{Catalysis}

\section{Anti-Markovnikov addition of dimethylhydrazine to phenylacetylene:}

Catalyst (6e)CuCl (0.01 mmol, $6 \mathrm{mg}$ ), potassium tert-butoxide (0.01 mmol, $1 \mathrm{mg}), \mathrm{Me}_{2} \mathrm{NNH}_{2}$ (301 mg, $5 \mathrm{mmol})$ and PhCCH (1 mmol, $102 \mathrm{mg})$ were loaded to a J-Young tube, and the reaction solution was heated to $120{ }^{\circ} \mathrm{C}$ for 24 hours. The reaction solution was cooled to room temperature and decanted to a round bottom flask. The volatiles were removed in vacuo. The crude product was dissolved in $\mathrm{C}_{6} \mathrm{D}_{6}$ using $\mathrm{PhCH}_{2} \mathrm{OMe}$ as standard $(24 \mathrm{mg}, 0.2 \mathrm{mmol})$ and the yield was determined by ${ }^{1} \mathrm{H}$ NMR.

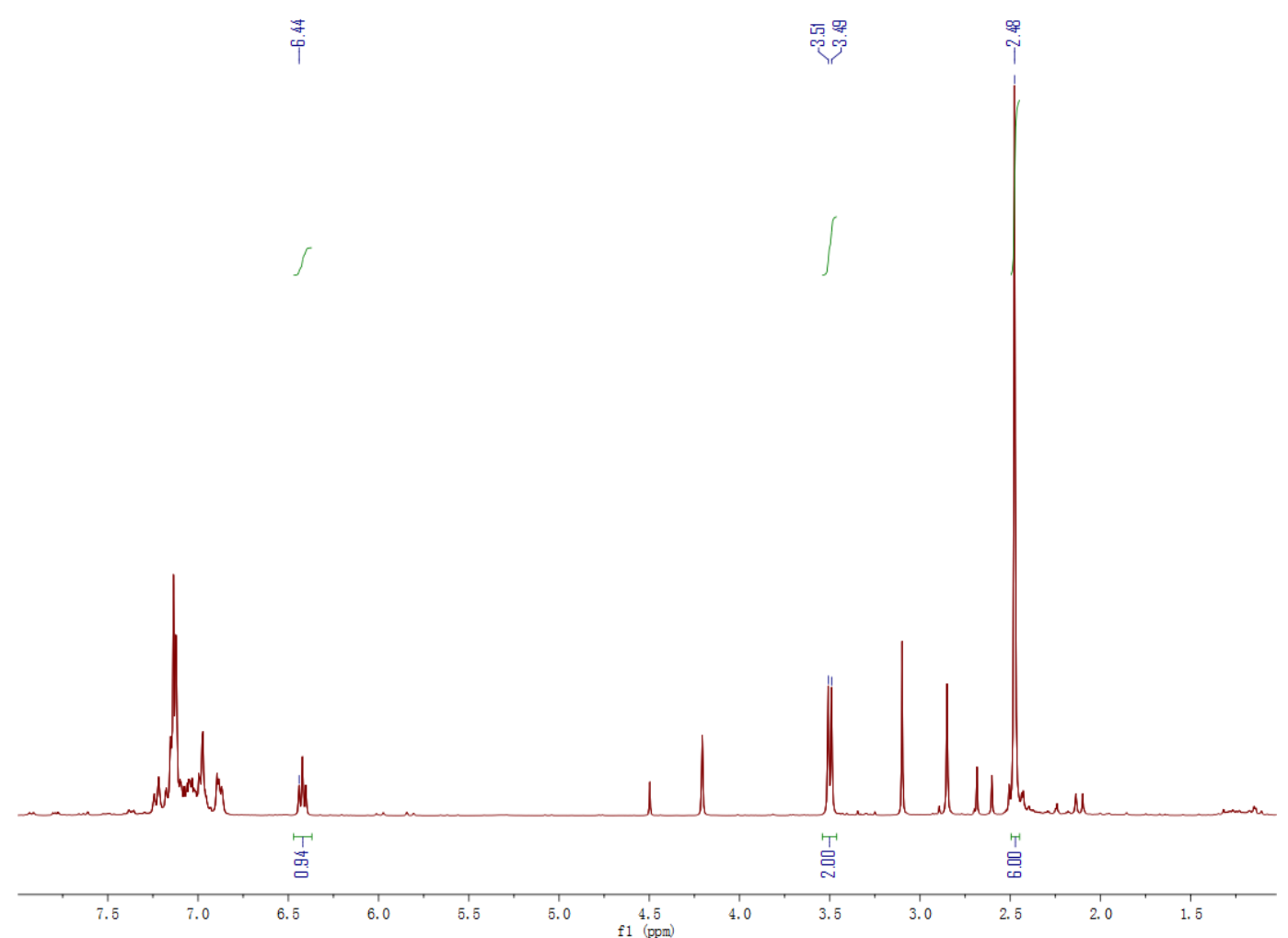

Figure S62. ${ }^{1} \mathrm{H}$ NMR of anti-Markovnikov addition of dimethylhydrazine to phenylacetylene with catalyst $(\mathbf{6 e}) \mathbf{C u O} \mathbf{O}^{t} \mathbf{B u}$.

\section{Hydroarylation reaction}

Catalyst $(0.01 \mathrm{mmol}), \mathrm{KB}\left(\mathrm{C}_{6} \mathrm{~F}_{5}\right)_{4}(14 \mathrm{mg}, 0.02 \mathrm{mmol}), N, N$-dimethyl aniline $(24 \mathrm{mg}, 0.20$ mmol) and $\alpha$-methyl styrene (24 $\mathrm{mg}, 0.20 \mathrm{mmol}$ ) were loaded to a J-Young tube. The solution was heated to $120{ }^{\circ} \mathrm{C}$ for 1 hour, and cooled down to room temperature. The conversion was determined via integration of the methyl peaks of the product and $\alpha$-methyl styrene in the ${ }^{1} \mathrm{H}$ NMR spectrum. Then, the J-Young tube was again heated to $120^{\circ} \mathrm{C}$ for $11 \mathrm{~h}$, and the conversion was again determined via ${ }^{1} \mathrm{H}$ NMR. 


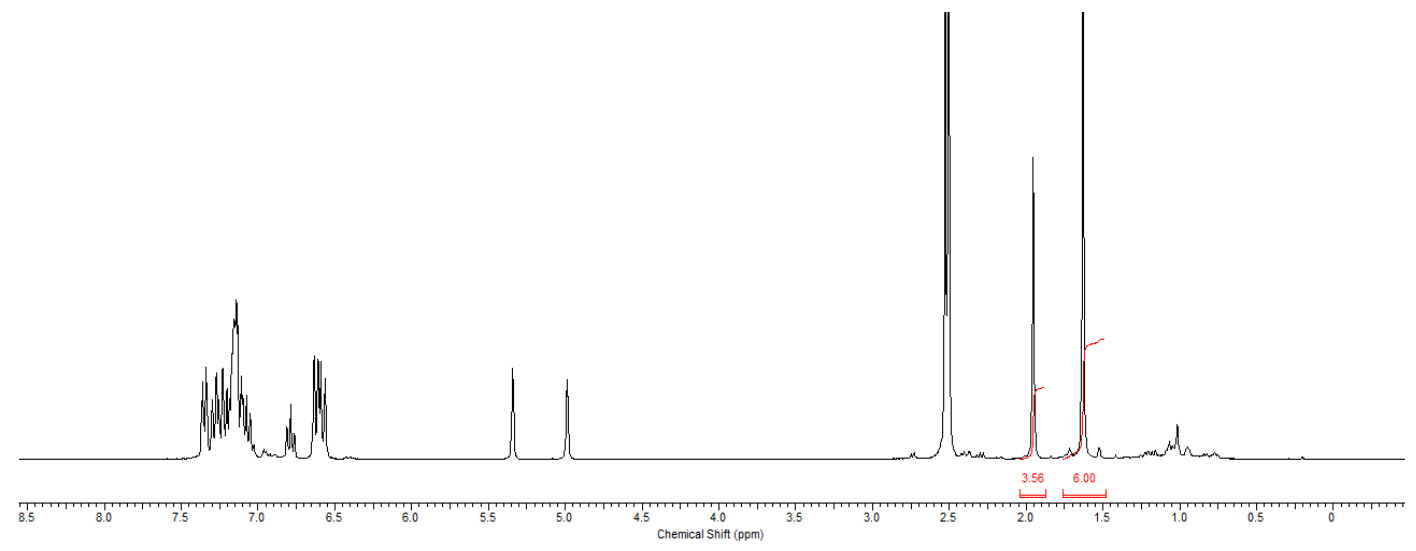

Figure S63. ${ }^{1} \mathrm{H}$ NMR of hydroarylation of $\alpha$-methyl styrene with $N, N$-dimethyl aniline in presence of $0.01 \mathrm{mmol}(\mathbf{6 e}) \mathbf{A u C l}$ after $1 \mathrm{~h}$ (45\% conversion).

\section{Solid State Structures}

\subsection{Copper Complex (6d)CuCl}

Single crystals of the $\left[\mathrm{C}_{22} \mathrm{H}_{36} \mathrm{ClCuN}_{2}\right](\mathbf{6 d}) \mathbf{C u C l}$ were grown by slow diffusion of hexane into a solution in benzene. A suitable crystal was selected and mounted on a 'Bruker APEX-II CCD' diffractometer. The crystal was kept at $100 \mathrm{~K}$ during data collection. Using Olex $2,{ }^{8}$ the structure was solved with the ShelXS ${ }^{9}$ structure solution program using Direct Methods and refined with the ShelXL ${ }^{9}$ refinement package using Least Squares minimization. The structure was twinned and refined using the twinned data refinement Scales: 0.703(19) $0.297(19)$

Table S 6.1.1. Crystal data and structure refinement for (6d)CuCl.

$\begin{array}{cc}\text { Identification code } & (6 \mathrm{~d}) \mathrm{CuCl} \\ \text { Empirical formula } & \mathrm{C}_{22} \mathrm{H}_{36} \mathrm{ClCuN}_{2} \\ \text { Formula weight } & 427.52 \\ \text { Temperature/K } & 100.0 \\ \text { Crystal system } & \text { monoclinic } \\ \text { Space group } & \mathrm{P} 2{ }_{1} \\ \mathrm{a} / \AA & 8.4234(3) \\ \mathrm{b} / \AA & 16.0108(6) \\ \mathrm{c} / \AA & 8.5636(3) \\ \alpha /{ }^{\circ} & 90 \\ \beta /{ }^{\circ} & 101.320(2) \\ \gamma /{ }^{\circ} & 90 \\ \mathrm{Volume} / \AA^{3} & 1132.47(7) \\ \mathrm{Z} & 2 \\ \rho_{\text {calc }} / \mathrm{cm}^{3} & 1.254 \\ \mu / \mathrm{mm}^{-1} & 1.090 \\ \mathrm{~F}(000) & 456.0\end{array}$




$$
\begin{gathered}
\text { Crystal size } / \mathrm{mm}^{3} \\
\text { Radiation }
\end{gathered}
$$

$2 \Theta$ range for data collection/ ${ }^{\circ}$

Index ranges

Reflections collected

Independent reflections

Data/restraints/parameters

Goodness-of-fit on $\mathrm{F}^{2}$

Final $\mathrm{R}$ indexes $[\mathrm{I}>=2 \sigma(\mathrm{I})]$

Final R indexes [all data]

Largest diff. peak/hole / e $\AA^{-3}$

Flack parameter
$0.18 \times 0.16 \times 0.112$

$\operatorname{MoK} \alpha(\lambda=0.71073)$

4.852 to 50.792

$-10 \leq \mathrm{h} \leq 8,-18 \leq \mathrm{k} \leq 19,-10 \leq 1 \leq 8$

$$
6287
$$

$3784\left[\mathrm{R}_{\mathrm{int}}=0.0202, \mathrm{R}_{\text {sigma }}=0.0396\right]$

$3784 / 1 / 245$

1.021

$\mathrm{R}_{1}=0.0340, \mathrm{wR}_{2}=0.0796$

$\mathrm{R}_{1}=0.0380, \mathrm{wR}_{2}=0.0822$

$0.52 /-0.23$

$0.297(19)$

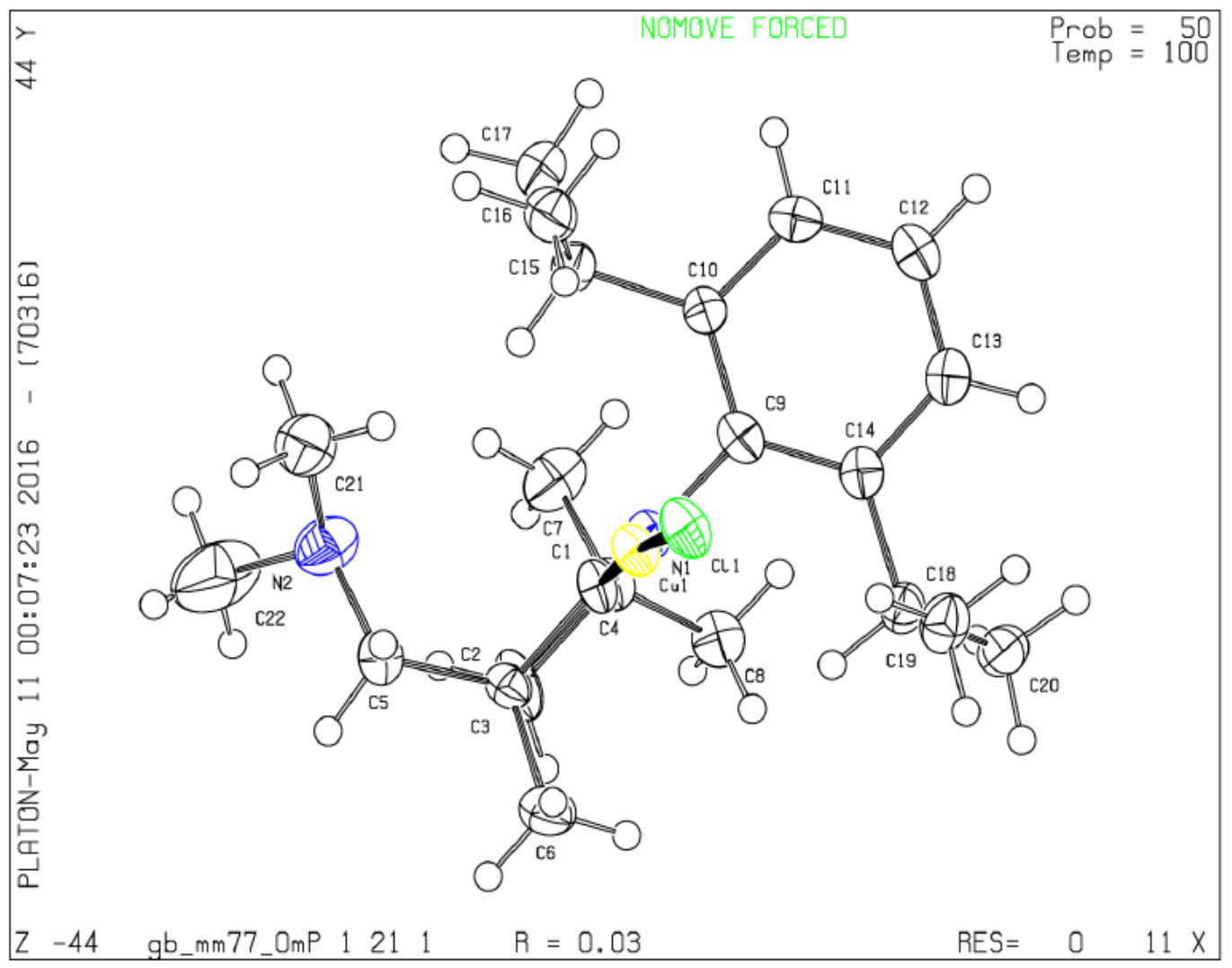

Table S 6.1.2. Bond lengths for (6d)CuCl.

$\begin{array}{cccccc}\text { Atom } & \text { Atom } & \text { Bond Length / } \AA & \text { Atom } & \text { Atom } & \text { Bond Length / } \AA \\ \mathrm{Cu} 1 & \mathrm{C} 11 & 2.1068(12) & \mathrm{C} 17 & \mathrm{H} 17 \mathrm{~B} & 0.98 \\ \mathrm{Cu} 1 & \mathrm{C} 1 & 1.870(4) & \mathrm{C} 17 & \mathrm{H} 17 \mathrm{C} & 0.98 \\ \mathrm{~N} 1 & \mathrm{C} 9 & 1.460(6) & \mathrm{C} 11 & \mathrm{H} 11 & 0.95 \\ \mathrm{~N} 1 & \mathrm{C} 1 & 1.310(5) & \mathrm{C} 18 & \mathrm{H} 18 & 1 \\ \mathrm{~N} 1 & \mathrm{C} 4 & 1.530(5) & \mathrm{C} 18 & \mathrm{C} 19 & 1.535(7)\end{array}$




\begin{tabular}{|c|c|c|c|c|c|}
\hline $\mathrm{C} 9$ & $\mathrm{C} 10$ & $1.404(7)$ & $\mathrm{C} 4$ & $\mathrm{C} 3$ & $1.535(6)$ \\
\hline C9 & $\mathrm{C} 14$ & $1.398(6)$ & $\mathrm{C} 4$ & $\mathrm{C} 7$ & $1.519(7)$ \\
\hline $\mathrm{C} 15$ & H15 & 1 & $\mathrm{C} 4$ & $\mathrm{C} 8$ & $1.515(6)$ \\
\hline $\mathrm{C} 15$ & $\mathrm{C} 10$ & $1.530(6)$ & $\mathrm{C} 3$ & $\mathrm{H} 3 \mathrm{~A}$ & 0.99 \\
\hline $\mathrm{C} 15$ & $\mathrm{C} 17$ & $1.517(6)$ & $\mathrm{C} 3$ & H3B & 0.99 \\
\hline $\mathrm{C} 15$ & $\mathrm{C} 16$ & $1.541(7)$ & $\mathrm{C} 6$ & H6A & 0.98 \\
\hline $\mathrm{C} 2$ & $\mathrm{C} 5$ & $1.541(7)$ & $\mathrm{C} 6$ & H6B & 0.98 \\
\hline $\mathrm{C} 2$ & $\mathrm{C} 1$ & $1.516(5)$ & $\mathrm{C} 6$ & H6C & 0.98 \\
\hline $\mathrm{C} 2$ & $\mathrm{C} 3$ & $1.545(6)$ & C19 & H19A & 0.98 \\
\hline $\mathrm{C} 2$ & C6 & $1.525(9)$ & C19 & H19B & 0.98 \\
\hline $\mathrm{C} 10$ & $\mathrm{C} 11$ & $1.402(7)$ & C19 & H19C & 0.98 \\
\hline $\mathrm{N} 2$ & $\mathrm{C} 5$ & $1.465(7)$ & $\mathrm{C} 16$ & H16A & 0.98 \\
\hline N2 & $\mathrm{C} 21$ & $1.453(7)$ & $\mathrm{C} 16$ & H16B & 0.98 \\
\hline $\mathrm{N} 2$ & $\mathrm{C} 22$ & $1.464(8)$ & $\mathrm{C} 16$ & $\mathrm{H} 16 \mathrm{C}$ & 0.98 \\
\hline C14 & $\mathrm{C} 13$ & $1.401(6)$ & $\mathrm{C} 7$ & H7A & 0.98 \\
\hline $\mathrm{C} 14$ & $\mathrm{C} 18$ & $1.522(7)$ & $\mathrm{C} 7$ & H7B & 0.98 \\
\hline $\mathrm{C} 13$ & $\mathrm{H} 13$ & 0.95 & $\mathrm{C} 7$ & $\mathrm{H} 7 \mathrm{C}$ & 0.98 \\
\hline $\mathrm{C} 13$ & $\mathrm{C} 12$ & $1.372(7)$ & $\mathrm{C} 8$ & H8A & 0.98 \\
\hline $\mathrm{C} 12$ & H12 & 0.95 & $\mathrm{C} 8$ & H8B & 0.98 \\
\hline $\mathrm{C} 12$ & $\mathrm{C} 11$ & $1.378(7)$ & $\mathrm{C} 8$ & $\mathrm{H} 8 \mathrm{C}$ & 0.98 \\
\hline $\mathrm{C} 5$ & H5A & 0.99 & $\mathrm{C} 21$ & $\mathrm{H} 21 \mathrm{~A}$ & 0.98 \\
\hline $\mathrm{C} 5$ & H5B & 0.99 & $\mathrm{C} 21$ & $\mathrm{H} 21 \mathrm{~B}$ & 0.98 \\
\hline $\mathrm{C} 20$ & $\mathrm{H} 20 \mathrm{~A}$ & 0.98 & $\mathrm{C} 21$ & $\mathrm{H} 21 \mathrm{C}$ & 0.98 \\
\hline $\mathrm{C} 20$ & $\mathrm{H} 20 \mathrm{~B}$ & 0.98 & $\mathrm{C} 22$ & $\mathrm{H} 22 \mathrm{~A}$ & 0.98 \\
\hline $\mathrm{C} 20$ & $\mathrm{H} 20 \mathrm{C}$ & 0.98 & $\mathrm{C} 22$ & $\mathrm{H} 22 \mathrm{~B}$ & 0.98 \\
\hline $\mathrm{C} 20$ & $\mathrm{C} 18$ & $1.533(7)$ & $\mathrm{C} 22$ & $\mathrm{H} 22 \mathrm{C}$ & 0.98 \\
\hline $\mathrm{C} 17$ & H17A & 0.98 & & & \\
\hline
\end{tabular}

Table S 6.1.3. Bond angles for (6d) CuCl.

$\begin{array}{cccccccc}\text { Atom } & \text { Atom } & \text { Atom } & \text { Angle / } & \text { Atom } & \text { Atom } & \text { Atom } & \text { Angle / } \\ \text { C1 } & \text { Cu1 } & \text { C11 } & 174.27(18) & \text { C14 } & \text { C18 } & \text { H18 } & 107.9 \\ \text { C9 } & \text { N1 } & \text { C4 } & 121.4(3) & \text { C14 } & \text { C18 } & \text { C19 } & 110.0(4) \\ \text { C1 } & \text { N1 } & \text { C9 } & 122.1(4) & \text { C20 } & \text { C18 } & \text { H18 } & 107.9 \\ \text { C1 } & \text { N1 } & \text { C4 } & 116.5(3) & \text { C20 } & \text { C18 } & \text { C19 } & 109.8(4) \\ \text { C10 } & \text { C9 } & \text { N1 } & 118.4(4) & \text { C19 } & \text { C18 } & \text { H18 } & 107.9 \\ \text { C14 } & \text { C9 } & \text { N1 } & 119.1(4) & \text { N1 } & \text { C4 } & \text { C3 } & 100.7(3) \\ \text { C14 } & \text { C9 } & \text { C10 } & 122.4(4) & \text { C7 } & \text { C4 } & \text { N1 } & 110.3(4) \\ \text { C10 } & \text { C15 } & \text { H15 } & 108.2 & \text { C7 } & \text { C4 } & \text { C3 } & 112.2(4) \\ \text { C10 } & \text { C15 } & \text { C16 } & 108.4(4) & \text { C8 } & \text { C4 } & \text { N1 } & 110.6(3) \\ \text { C17 } & \text { C15 } & \text { H15 } & 108.2 & \text { C8 } & \text { C4 } & \text { C3 } & 112.7(4) \\ \text { C17 } & \text { C15 } & \text { C10 } & 113.3(4) & \text { C8 } & \text { C4 } & \text { C7 } & 110.0(4) \\ \text { C17 } & \text { C15 } & \text { C16 } & 110.5(4) & \text { C2 } & \text { C3 } & \text { H3A } & 110 \\ \text { C16 } & \text { C15 } & \text { H15 } & 108.2 & \text { C2 } & \text { C3 } & \text { H3B } & 110\end{array}$




\begin{tabular}{|c|c|c|c|c|c|c|c|}
\hline $\mathrm{C} 5$ & $\mathrm{C} 2$ & $\mathrm{C} 3$ & $113.2(5)$ & $\mathrm{C} 4$ & $\mathrm{C} 3$ & $\mathrm{C} 2$ & $108.6(3)$ \\
\hline $\mathrm{C} 1$ & $\mathrm{C} 2$ & $\mathrm{C} 5$ & 111.1(4) & $\mathrm{C} 4$ & $\mathrm{C} 3$ & $\mathrm{H} 3 \mathrm{~A}$ & 110 \\
\hline $\mathrm{C} 1$ & $\mathrm{C} 2$ & $\mathrm{C} 3$ & $104.7(3)$ & $\mathrm{C} 4$ & $\mathrm{C} 3$ & H3B & 110 \\
\hline $\mathrm{C} 1$ & $\mathrm{C} 2$ & C6 & $107.5(5)$ & $\mathrm{H} 3 \mathrm{~A}$ & $\mathrm{C} 3$ & $\mathrm{H} 3 \mathrm{~B}$ & 108.4 \\
\hline C6 & $\mathrm{C} 2$ & C5 & $107.5(4)$ & $\mathrm{C} 2$ & $\mathrm{C} 6$ & H6A & 109.5 \\
\hline C6 & $\mathrm{C} 2$ & $\mathrm{C} 3$ & $112.9(5)$ & $\mathrm{C} 2$ & $\mathrm{C} 6$ & H6B & 109.5 \\
\hline C9 & $\mathrm{C} 10$ & $\mathrm{C} 15$ & $124.3(4)$ & $\mathrm{C} 2$ & $\mathrm{C} 6$ & $\mathrm{H} 6 \mathrm{C}$ & 109.5 \\
\hline $\mathrm{C} 11$ & $\mathrm{C} 10$ & $\mathrm{C} 9$ & 117.1(4) & H6A & $\mathrm{C} 6$ & H6B & 109.5 \\
\hline $\mathrm{C} 11$ & $\mathrm{C} 10$ & $\mathrm{C} 15$ & $118.3(4)$ & H6A & $\mathrm{C} 6$ & $\mathrm{H} 6 \mathrm{C}$ & 109.5 \\
\hline $\mathrm{C} 21$ & $\mathrm{~N} 2$ & $\mathrm{C} 5$ & $112.0(4)$ & H6B & $\mathrm{C} 6$ & $\mathrm{H} 6 \mathrm{C}$ & 109.5 \\
\hline $\mathrm{C} 21$ & $\mathrm{~N} 2$ & $\mathrm{C} 22$ & 111.3(6) & $\mathrm{C} 18$ & C19 & H19A & 109.5 \\
\hline $\mathrm{C} 22$ & $\mathrm{~N} 2$ & $\mathrm{C} 5$ & $112.0(5)$ & $\mathrm{C} 18$ & $\mathrm{C} 19$ & H19B & 109.5 \\
\hline $\mathrm{C} 9$ & $\mathrm{C} 14$ & $\mathrm{C} 13$ & $117.4(4)$ & $\mathrm{C} 18$ & C19 & H19C & 109.5 \\
\hline C9 & $\mathrm{C} 14$ & $\mathrm{C} 18$ & $123.9(4)$ & H19A & $\mathrm{C} 19$ & H19B & 109.5 \\
\hline $\mathrm{C} 13$ & $\mathrm{C} 14$ & $\mathrm{C} 18$ & $118.4(4)$ & H19A & C19 & $\mathrm{H} 19 \mathrm{C}$ & 109.5 \\
\hline $\mathrm{C} 14$ & C13 & H13 & 119.4 & H19B & C19 & $\mathrm{H} 19 \mathrm{C}$ & 109.5 \\
\hline $\mathrm{C} 12$ & $\mathrm{C} 13$ & $\mathrm{C} 14$ & $121.3(4)$ & $\mathrm{C} 15$ & $\mathrm{C} 16$ & H16A & 109.5 \\
\hline $\mathrm{C} 12$ & $\mathrm{C} 13$ & H13 & 119.4 & $\mathrm{C} 15$ & $\mathrm{C} 16$ & H16B & 109.5 \\
\hline $\mathrm{C} 13$ & $\mathrm{C} 12$ & H12 & 119.9 & $\mathrm{C} 15$ & $\mathrm{C} 16$ & $\mathrm{H} 16 \mathrm{C}$ & 109.5 \\
\hline $\mathrm{C} 13$ & $\mathrm{C} 12$ & $\mathrm{C} 11$ & $120.3(4)$ & H16A & $\mathrm{C} 16$ & H16B & 109.5 \\
\hline $\mathrm{C} 11$ & $\mathrm{C} 12$ & H12 & 119.9 & H16A & $\mathrm{C} 16$ & $\mathrm{H} 16 \mathrm{C}$ & 109.5 \\
\hline $\mathrm{C} 2$ & $\mathrm{C} 5$ & $\mathrm{H} 5 \mathrm{~A}$ & 108.9 & H16B & $\mathrm{C} 16$ & $\mathrm{H} 16 \mathrm{C}$ & 109.5 \\
\hline $\mathrm{C} 2$ & $\mathrm{C} 5$ & H5B & 108.9 & $\mathrm{C} 4$ & $\mathrm{C} 7$ & H7A & 109.5 \\
\hline N2 & $\mathrm{C} 5$ & $\mathrm{C} 2$ & $113.5(4)$ & $\mathrm{C} 4$ & $\mathrm{C} 7$ & $\mathrm{H} 7 \mathrm{~B}$ & 109.5 \\
\hline N2 & $\mathrm{C} 5$ & $\mathrm{H} 5 \mathrm{~A}$ & 108.9 & $\mathrm{C} 4$ & $\mathrm{C} 7$ & $\mathrm{H} 7 \mathrm{C}$ & 109.5 \\
\hline $\mathrm{N} 2$ & $\mathrm{C} 5$ & H5B & 108.9 & H7A & $\mathrm{C} 7$ & H7B & 109.5 \\
\hline $\mathrm{H} 5 \mathrm{~A}$ & $\mathrm{C} 5$ & H5B & 107.7 & H7A & $\mathrm{C} 7$ & $\mathrm{H} 7 \mathrm{C}$ & 109.5 \\
\hline N1 & $\mathrm{C} 1$ & $\mathrm{Cu} 1$ & 125.1(3) & H7B & $\mathrm{C} 7$ & $\mathrm{H} 7 \mathrm{C}$ & 109.5 \\
\hline N1 & $\mathrm{C} 1$ & $\mathrm{C} 2$ & $109.5(4)$ & $\mathrm{C} 4$ & $\mathrm{C} 8$ & $\mathrm{H} 8 \mathrm{~A}$ & 109.5 \\
\hline $\mathrm{C} 2$ & $\mathrm{C} 1$ & $\mathrm{Cu} 1$ & $125.3(3)$ & $\mathrm{C} 4$ & $\mathrm{C} 8$ & H8B & 109.5 \\
\hline $\mathrm{H} 20 \mathrm{~A}$ & $\mathrm{C} 20$ & H20B & 109.5 & $\mathrm{C} 4$ & $\mathrm{C} 8$ & $\mathrm{H} 8 \mathrm{C}$ & 109.5 \\
\hline $\mathrm{H} 20 \mathrm{~A}$ & $\mathrm{C} 20$ & $\mathrm{H} 20 \mathrm{C}$ & 109.5 & H8A & $\mathrm{C} 8$ & $\mathrm{H} 8 \mathrm{~B}$ & 109.5 \\
\hline $\mathrm{H} 20 \mathrm{~B}$ & $\mathrm{C} 20$ & $\mathrm{H} 20 \mathrm{C}$ & 109.5 & H8A & $\mathrm{C} 8$ & $\mathrm{H} 8 \mathrm{C}$ & 109.5 \\
\hline $\mathrm{C} 18$ & $\mathrm{C} 20$ & $\mathrm{H} 20 \mathrm{~A}$ & 109.5 & H8B & $\mathrm{C} 8$ & $\mathrm{H} 8 \mathrm{C}$ & 109.5 \\
\hline $\mathrm{C} 18$ & $\mathrm{C} 20$ & $\mathrm{H} 20 \mathrm{~B}$ & 109.5 & $\mathrm{~N} 2$ & $\mathrm{C} 21$ & $\mathrm{H} 21 \mathrm{~A}$ & 109.5 \\
\hline $\mathrm{C} 18$ & $\mathrm{C} 20$ & $\mathrm{H} 20 \mathrm{C}$ & 109.5 & $\mathrm{~N} 2$ & $\mathrm{C} 21$ & $\mathrm{H} 21 \mathrm{~B}$ & 109.5 \\
\hline $\mathrm{C} 15$ & $\mathrm{C} 17$ & H17A & 109.5 & $\mathrm{~N} 2$ & $\mathrm{C} 21$ & $\mathrm{H} 21 \mathrm{C}$ & 109.5 \\
\hline $\mathrm{C} 15$ & $\mathrm{C} 17$ & H17B & 109.5 & $\mathrm{H} 21 \mathrm{~A}$ & $\mathrm{C} 21$ & $\mathrm{H} 21 \mathrm{~B}$ & 109.5 \\
\hline $\mathrm{C} 15$ & $\mathrm{C} 17$ & $\mathrm{H} 17 \mathrm{C}$ & 109.5 & $\mathrm{H} 21 \mathrm{~A}$ & $\mathrm{C} 21$ & $\mathrm{H} 21 \mathrm{C}$ & 109.5 \\
\hline H17A & $\mathrm{C} 17$ & H17B & 109.5 & $\mathrm{H} 21 \mathrm{~B}$ & $\mathrm{C} 21$ & $\mathrm{H} 21 \mathrm{C}$ & 109.5 \\
\hline H17A & $\mathrm{C} 17$ & H17C & 109.5 & $\mathrm{~N} 2$ & $\mathrm{C} 22$ & $\mathrm{H} 22 \mathrm{~A}$ & 109.5 \\
\hline H17B & $\mathrm{C} 17$ & $\mathrm{H} 17 \mathrm{C}$ & 109.5 & $\mathrm{~N} 2$ & $\mathrm{C} 22$ & $\mathrm{H} 22 \mathrm{~B}$ & 109.5 \\
\hline $\mathrm{C} 10$ & $\mathrm{C} 11$ & H11 & 119.4 & $\mathrm{~N} 2$ & $\mathrm{C} 22$ & $\mathrm{H} 22 \mathrm{C}$ & 109.5 \\
\hline $\mathrm{C} 12$ & $\mathrm{C} 11$ & $\mathrm{C} 10$ & $121.3(4)$ & $\mathrm{H} 22 \mathrm{~A}$ & $\mathrm{C} 22$ & $\mathrm{H} 22 \mathrm{~B}$ & 109.5 \\
\hline
\end{tabular}




$\begin{array}{lllcllll}\mathrm{C} 12 & \mathrm{C} 11 & \mathrm{H} 11 & 119.4 & \mathrm{H} 22 \mathrm{~A} & \mathrm{C} 22 & \mathrm{H} 22 \mathrm{C} & 109.5 \\ \mathrm{C} 14 & \mathrm{C} 18 & \mathrm{C} 20 & 113.3(4) & \mathrm{H} 22 \mathrm{~B} & \mathrm{C} 22 & \mathrm{H} 22 \mathrm{C} & 109.5\end{array}$

Table S 6.1.4. Torsion angles for $(6 \mathrm{~d}) \mathrm{CuCl}$.

\begin{tabular}{|c|c|c|c|c|}
\hline Atom & Atom & Atom & Atom & Torsion Angle ${ }^{\circ}$ \\
\hline N1 & $\mathrm{C} 9$ & $\mathrm{C} 10$ & $\mathrm{C} 15$ & $9.5(7)$ \\
\hline $\mathrm{N} 1$ & C9 & $\mathrm{C} 10$ & $\mathrm{C} 11$ & $-176.0(4)$ \\
\hline $\mathrm{N} 1$ & C9 & C14 & $\mathrm{C} 13$ & $175.6(4)$ \\
\hline $\mathrm{N} 1$ & $\mathrm{C} 9$ & C14 & $\mathrm{C} 18$ & $-10.4(6)$ \\
\hline $\mathrm{N} 1$ & $\mathrm{C} 4$ & $\mathrm{C} 3$ & $\mathrm{C} 2$ & $0.5(6)$ \\
\hline $\mathrm{C} 9$ & $\mathrm{~N} 1$ & $\mathrm{C} 1$ & $\mathrm{Cu} 1$ & $-2.3(7)$ \\
\hline $\mathrm{C} 9$ & $\mathrm{~N} 1$ & $\mathrm{C} 1$ & $\mathrm{C} 2$ & $-178.4(4)$ \\
\hline $\mathrm{C} 9$ & $\mathrm{~N} 1$ & $\mathrm{C} 4$ & $\mathrm{C} 3$ & $178.0(4)$ \\
\hline $\mathrm{C} 9$ & $\mathrm{~N} 1$ & $\mathrm{C} 4$ & $\mathrm{C} 7$ & $-63.4(5)$ \\
\hline $\mathrm{C} 9$ & $\mathrm{~N} 1$ & $\mathrm{C} 4$ & $\mathrm{C} 8$ & $58.6(5)$ \\
\hline C9 & $\mathrm{C} 10$ & $\mathrm{C} 11$ & $\mathrm{C} 12$ & $-1.3(7)$ \\
\hline $\mathrm{C} 9$ & $\mathrm{C} 14$ & $\mathrm{C} 13$ & $\mathrm{C} 12$ & $2.1(6)$ \\
\hline $\mathrm{C} 9$ & $\mathrm{C} 14$ & $\mathrm{C} 18$ & $\mathrm{C} 20$ & $130.4(5)$ \\
\hline $\mathrm{C} 9$ & $\mathrm{C} 14$ & $\mathrm{C} 18$ & $\mathrm{C} 19$ & $-106.4(5)$ \\
\hline $\mathrm{C} 15$ & $\mathrm{C} 10$ & $\mathrm{C} 11$ & $\mathrm{C} 12$ & $173.6(4)$ \\
\hline $\mathrm{C} 10$ & C9 & $\mathrm{C} 14$ & $\mathrm{C} 13$ & $-5.4(6)$ \\
\hline $\mathrm{C} 10$ & C9 & C14 & $\mathrm{C} 18$ & $168.6(4)$ \\
\hline $\mathrm{C} 14$ & C9 & $\mathrm{C} 10$ & $\mathrm{C} 15$ & $-169.5(4)$ \\
\hline $\mathrm{C} 14$ & C9 & $\mathrm{C} 10$ & $\mathrm{C} 11$ & $5.1(7)$ \\
\hline C14 & $\mathrm{C} 13$ & $\mathrm{C} 12$ & $\mathrm{C} 11$ & $1.5(7)$ \\
\hline $\mathrm{C} 13$ & $\mathrm{C} 14$ & $\mathrm{C} 18$ & $\mathrm{C} 20$ & $-55.7(6)$ \\
\hline $\mathrm{C} 13$ & $\mathrm{C} 14$ & $\mathrm{C} 18$ & $\mathrm{C} 19$ & $67.6(5)$ \\
\hline $\mathrm{C} 13$ & $\mathrm{C} 12$ & $\mathrm{C} 11$ & $\mathrm{C} 10$ & $-1.9(7)$ \\
\hline $\mathrm{C} 5$ & $\mathrm{C} 2$ & $\mathrm{C} 1$ & $\mathrm{Cu} 1$ & $62.1(7)$ \\
\hline $\mathrm{C} 5$ & $\mathrm{C} 2$ & $\mathrm{C} 1$ & N1 & $-121.8(4)$ \\
\hline $\mathrm{C} 5$ & $\mathrm{C} 2$ & $\mathrm{C} 3$ & $\mathrm{C} 4$ & $120.4(5)$ \\
\hline $\mathrm{C} 1$ & $\mathrm{~N} 1$ & $\mathrm{C} 9$ & $\mathrm{C} 10$ & $-91.4(6)$ \\
\hline $\mathrm{C} 1$ & $\mathrm{~N} 1$ & C9 & $\mathrm{C} 14$ & $87.6(5)$ \\
\hline $\mathrm{C} 1$ & N1 & $\mathrm{C} 4$ & $\mathrm{C} 3$ & $0.0(5)$ \\
\hline $\mathrm{C} 1$ & $\mathrm{~N} 1$ & $\mathrm{C} 4$ & $\mathrm{C} 7$ & $118.6(5)$ \\
\hline $\mathrm{C} 1$ & N1 & $\mathrm{C} 4$ & $\mathrm{C} 8$ & $-119.4(5)$ \\
\hline $\mathrm{C} 1$ & $\mathrm{C} 2$ & $\mathrm{C} 5$ & $\mathrm{~N} 2$ & $68.6(5)$ \\
\hline $\mathrm{C} 1$ & $\mathrm{C} 2$ & $\mathrm{C} 3$ & $\mathrm{C} 4$ & $-0.7(7)$ \\
\hline $\mathrm{C} 17$ & $\mathrm{C} 15$ & $\mathrm{C} 10$ & C9 & $-130.7(5)$ \\
\hline $\mathrm{C} 17$ & $\mathrm{C} 15$ & $\mathrm{C} 10$ & $\mathrm{C} 11$ & $54.8(6)$ \\
\hline $\mathrm{C} 18$ & $\mathrm{C} 14$ & $\mathrm{C} 13$ & $\mathrm{C} 12$ & $-172.3(4)$ \\
\hline $\mathrm{C} 4$ & N1 & $\mathrm{C} 9$ & $\mathrm{C} 10$ & $90.7(5)$ \\
\hline $\mathrm{C} 4$ & N1 & C9 & C14 & $-90.3(5)$ \\
\hline
\end{tabular}




$\begin{array}{ccccc}\mathrm{C} 4 & \mathrm{~N} 1 & \mathrm{C} 1 & \mathrm{C} 1 & 175.7(3) \\ \mathrm{C} 4 & \mathrm{~N} 1 & \mathrm{C} 1 & \mathrm{C} 2 & -0.4(6) \\ \mathrm{C} 3 & \mathrm{C} 2 & \mathrm{C} 5 & \mathrm{~N} 2 & -48.8(6) \\ \mathrm{C} 3 & \mathrm{C} 2 & \mathrm{C} 1 & \mathrm{C} 1 & -175.4(4) \\ \mathrm{C} 3 & \mathrm{C} 2 & \mathrm{C} 1 & \mathrm{~N} 1 & 0.7(7) \\ \mathrm{C} 6 & \mathrm{C} 2 & \mathrm{C} 5 & \mathrm{~N} 2 & -174.1(4) \\ \mathrm{C} 6 & \mathrm{C} 2 & \mathrm{C} 1 & \mathrm{C} 1 & -55.2(6) \\ \mathrm{C} 6 & \mathrm{C} 2 & \mathrm{C} 1 & \mathrm{~N} 1 & 120.9(4) \\ \mathrm{C} 6 & \mathrm{C} 2 & \mathrm{C} 3 & \mathrm{C} 4 & -117.3(5) \\ \mathrm{C} 16 & \mathrm{C} 15 & \mathrm{C} 10 & \mathrm{C} 9 & 106.2(5) \\ \mathrm{C} 16 & \mathrm{C} 15 & \mathrm{C} 10 & \mathrm{C} 11 & -68.3(5) \\ \mathrm{C} 7 & \mathrm{C} 4 & \mathrm{C} 3 & \mathrm{C} 2 & -116.8(5) \\ \mathrm{C} 8 & \mathrm{C} 4 & \mathrm{C} 3 & \mathrm{C} 2 & 118.3(5) \\ \mathrm{C} 21 & \mathrm{~N} 2 & \mathrm{C} 5 & \mathrm{C} 2 & -114.9(5) \\ \mathrm{C} 22 & \mathrm{~N} 2 & \mathrm{C} 5 & \mathrm{C} 2 & 119.2(6)\end{array}$

\subsection{Gold Complex (6e)AuCl}

Single crystals of $\left[\mathrm{C}_{36} \mathrm{H}_{54} \mathrm{AuClN} \mathrm{N}_{2}\right](\mathbf{6 e}) \mathrm{AuCl}$ were grown by slow diffusion of hexane into a solution in benzene. A suitable crystal was selected and mounted on a 'Bruker APEX-II CCD' diffractometer. The crystal was kept at $100 \mathrm{~K}$ during data collection. Using Olex $2,{ }^{8}$ the structure was solved with the ShelXS ${ }^{9}$ structure solution program using Direct Methods and refined with the ShelXL ${ }^{9}$ refinement package using Least Squares minimization. The structure was twinned and refined using the twinned data refinement Scales: 0.5296(6) $0.4705(6)$

Table S6.2.1. Crystal data and structure refinement for (6e)AuCl.

$\begin{array}{ll}\text { Identification code } & (6 \mathrm{e}) \mathrm{AuCl} \\ \text { Empirical formula } & \mathrm{C}_{36} \mathrm{H}_{54} \mathrm{AuClN}_{2} \\ \text { Formula weight } & 747.23 \\ \text { Temperature/K } & 100.0 \\ \text { Crystal system } & \text { triclinic } \\ \text { Space group } & \mathrm{P}-1 \\ \mathrm{a} / \AA & 10.0911(7) \\ \mathrm{b} / \AA & 18.5601(14) \\ \mathrm{c} / \AA & 19.6959(14) \\ \alpha /{ }^{\circ} & 106.828(2) \\ \beta /{ }^{\circ} & 94.902(2) \\ \gamma /{ }^{\circ} & 98.618(2) \\ \mathrm{Volume} / \AA^{3} & 3458.5(4) \\ \mathrm{Z} & 4 \\ \rho_{\text {calc }} \mathrm{g} / \mathrm{cm}^{3} & 1.435 \\ \mu / \mathrm{mm}^{-1} & 4.356 \\ \mathrm{~F}(000) & 1520.0\end{array}$


Crystal size $/ \mathrm{mm}^{3}$

Radiation

$2 \Theta$ range for data collection ${ }^{\circ}$

Index ranges

Reflections collected

Independent reflections

Data/restraints/parameters

Goodness-of-fit on $\mathrm{F}^{2}$

Final $R$ indexes $[\mathrm{I}>=2 \sigma(\mathrm{I})]$

Final $\mathrm{R}$ indexes [all data]

Largest diff. peak/hole / e $\AA^{-3}$
$0.22 \times 0.22 \times 0.01$

$\operatorname{MoK} \alpha(\lambda=0.71073)$

2.18 to 50.83

$-12 \leq \mathrm{h} \leq 12,-22 \leq \mathrm{k} \leq 21,0 \leq 1 \leq 23$

16395

$16395\left[\mathrm{R}_{\text {int }}=?, \mathrm{R}_{\text {sigma }}=0.0710\right]$

$16395 / 30 / 738$

1.019

$\mathrm{R}_{1}=0.0405, \mathrm{wR}_{2}=0.0746$

$\mathrm{R}_{1}=0.0718, \mathrm{wR}_{2}=0.0836$

$1.49 /-0.91$

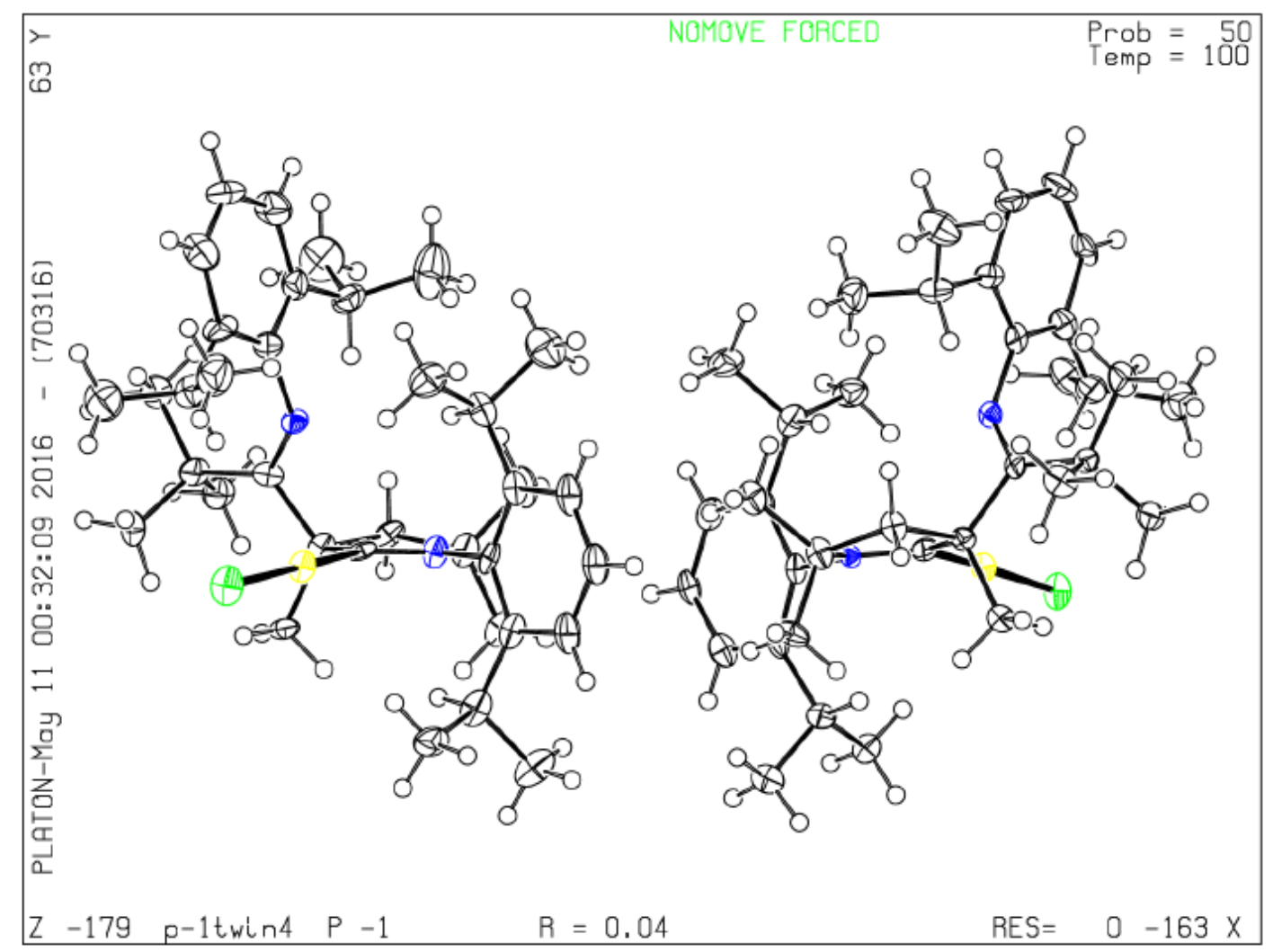

Table S6.2.2. Bond lengths for (6e)AuCl.

$\begin{array}{cccccc}\text { Atom } & \text { Atom } & \text { Bond Length / } \AA & \text { Atom } & \text { Atom } & \text { Bond Length / } \\ \text { Au1 } & \text { C11 } & 2.291(2) & \text { C21 } & \text { H21C } & 0.98 \\ \text { Au1 } & \text { C1 } & 1.984(8) & \text { C20 } & \text { H20A } & 0.98 \\ \text { Au1' } & \text { C11' } & 2.293(2) & \text { C20 } & \text { H20B } & 0.98 \\ \text { Au1' } & \text { C1' } & 1.974(8) & \text { C20 } & \text { H20C } & 0.98 \\ \text { N1 } & \text { C1 } & 1.285(10) & \text { C6 } & \text { C7 } & 1.539(11) \\ \text { N1 } & \text { C25 } & 1.461(10) & \text { C6 } & \text { C9 } & 1.535(12) \\ \text { N1 } & \text { C4 } & 1.527(10) & \text { C6 } & \text { C8 } & 1.542(11) \\ \text { N2 } & \text { C5 } & 1.266(10) & \text { C7 } & \text { H7A } & 0.98\end{array}$




\begin{tabular}{|c|c|c|c|c|c|}
\hline $\mathrm{N} 2$ & $\mathrm{C} 10$ & $1.406(10)$ & $\mathrm{C} 7$ & H7B & 0.98 \\
\hline $\mathrm{N} 1^{\prime}$ & $\mathrm{C} 25^{\prime}$ & $1.464(10)$ & $\mathrm{C} 7$ & $\mathrm{H} 7 \mathrm{C}$ & 0.98 \\
\hline $\mathrm{N} 1^{\prime}$ & $\mathrm{C} 1^{\prime}$ & $1.299(10)$ & C9 & H9A & 0.98 \\
\hline $\mathrm{N} 1^{\prime}$ & $\mathrm{C}^{\prime}$ & $1.550(10)$ & C9 & H9B & 0.98 \\
\hline $\mathrm{C} 25^{\prime}$ & $\mathrm{C} 30^{\prime}$ & $1.402(11)$ & $\mathrm{C} 9$ & $\mathrm{H} 9 \mathrm{C}$ & 0.98 \\
\hline $\mathrm{C} 25^{\prime}$ & $\mathrm{C} 26^{\prime}$ & $1.407(12)$ & $\mathrm{C} 8$ & H8A & 0.98 \\
\hline $\mathrm{N} 2^{\prime}$ & $\mathrm{C} 5^{\prime}$ & $1.259(10)$ & $\mathrm{C} 8$ & $\mathrm{H} 8 \mathrm{~B}$ & 0.98 \\
\hline N2' & $\mathrm{C} 10^{\prime}$ & $1.420(10)$ & $\mathrm{C} 8$ & $\mathrm{H} 8 \mathrm{C}$ & 0.98 \\
\hline $\mathrm{C} 11^{\prime}$ & $\mathrm{C} 10^{\prime}$ & $1.406(11)$ & $\mathrm{C} 1^{\prime}$ & $\mathrm{C} 2^{\prime}$ & $1.535(11)$ \\
\hline $\mathrm{C} 11^{\prime}$ & $\mathrm{C} 12^{\prime}$ & $1.389(11)$ & $\mathrm{C} 30^{\prime}$ & C34' & $1.514(11)$ \\
\hline C11' & C16' & $1.507(11)$ & $\mathrm{C} 30^{\prime}$ & $\mathrm{C} 29^{\prime}$ & $1.380(11)$ \\
\hline C19' & H19' & 1 & $\mathrm{C} 34^{\prime}$ & H34' & 1 \\
\hline C19' & $\mathrm{C} 15^{\prime}$ & $1.511(12)$ & C34' & $\mathrm{C} 36^{\prime}$ & $1.533(11)$ \\
\hline C19' & $\mathrm{C} 21^{\prime}$ & $1.534(11)$ & C34' & $\mathrm{C} 35^{\prime}$ & $1.532(10)$ \\
\hline C19' & $\mathrm{C} 20^{\prime}$ & $1.537(11)$ & $\mathrm{C} 36^{\prime}$ & H36D & 0.98 \\
\hline $\mathrm{C} 1$ & $\mathrm{C} 2$ & $1.533(11)$ & $\mathrm{C} 36^{\prime}$ & $\mathrm{H} 36 \mathrm{E}$ & 0.98 \\
\hline $\mathrm{C} 25$ & $\mathrm{C} 26$ & $1.397(12)$ & $\mathrm{C} 36^{\prime}$ & $\mathrm{H} 36 \mathrm{~F}$ & 0.98 \\
\hline $\mathrm{C} 25$ & $\mathrm{C} 30$ & $1.408(12)$ & $\mathrm{C} 35^{\prime}$ & H35D & 0.98 \\
\hline $\mathrm{C} 26$ & $\mathrm{C} 31$ & $1.510(13)$ & $\mathrm{C} 35^{\prime}$ & H35E & 0.98 \\
\hline $\mathrm{C} 26$ & $\mathrm{C} 27$ & $1.405(11)$ & $\mathrm{C} 35^{\prime}$ & $\mathrm{H} 35 \mathrm{~F}$ & 0.98 \\
\hline $\mathrm{C} 31$ & H31 & 1 & $\mathrm{C} 29^{\prime}$ & H29' & 0.95 \\
\hline $\mathrm{C} 31$ & $\mathrm{C} 33$ & $1.544(11)$ & $\mathrm{C} 29^{\prime}$ & $\mathrm{C} 28^{\prime}$ & $1.363(11)$ \\
\hline $\mathrm{C} 31$ & $\mathrm{C} 32$ & $1.529(11)$ & $\mathrm{C} 28^{\prime}$ & $\mathrm{H} 28^{\prime}$ & 0.95 \\
\hline $\mathrm{C} 33$ & H33A & 0.98 & $\mathrm{C} 28^{\prime}$ & $\mathrm{C} 27^{\prime}$ & $1.367(11)$ \\
\hline $\mathrm{C} 33$ & H33B & 0.98 & $\mathrm{C} 27^{\prime}$ & $\mathrm{H} 27^{\prime}$ & 0.95 \\
\hline $\mathrm{C} 33$ & $\mathrm{H} 33 \mathrm{C}$ & 0.98 & $\mathrm{C} 27^{\prime}$ & $\mathrm{C} 26^{\prime}$ & $1.389(11)$ \\
\hline $\mathrm{C} 32$ & $\mathrm{H} 32 \mathrm{~A}$ & 0.98 & $\mathrm{C} 26^{\prime}$ & C31' & $1.531(12)$ \\
\hline $\mathrm{C} 32$ & H32B & 0.98 & $\mathrm{C} 31^{\prime}$ & H31' & 1 \\
\hline $\mathrm{C} 32$ & $\mathrm{H} 32 \mathrm{C}$ & 0.98 & $\mathrm{C} 31^{\prime}$ & C33' & $1.519(11)$ \\
\hline $\mathrm{C} 27$ & $\mathrm{H} 27$ & 0.95 & $\mathrm{C} 31^{\prime}$ & C32' & $1.531(10)$ \\
\hline $\mathrm{C} 27$ & $\mathrm{C} 28$ & $1.350(12)$ & C33' & H33D & 0.98 \\
\hline $\mathrm{C} 28$ & $\mathrm{H} 28$ & 0.95 & C $33^{\prime}$ & $\mathrm{H} 33 \mathrm{E}$ & 0.98 \\
\hline $\mathrm{C} 28$ & $\mathrm{C} 29$ & $1.391(13)$ & C33' & $\mathrm{H} 33 \mathrm{~F}$ & 0.98 \\
\hline $\mathrm{C} 29$ & H29 & 0.95 & $\mathrm{C} 32^{\prime}$ & H32D & 0.98 \\
\hline $\mathrm{C} 29$ & $\mathrm{C} 30$ & $1.407(11)$ & $\mathrm{C} 32^{\prime}$ & $\mathrm{H} 32 \mathrm{E}$ & 0.98 \\
\hline $\mathrm{C} 30$ & $\mathrm{C} 34$ & $1.525(12)$ & $\mathrm{C} 32^{\prime}$ & $\mathrm{H} 32 \mathrm{~F}$ & 0.98 \\
\hline $\mathrm{C} 34$ & H34 & 1 & $\mathrm{C} 4{ }^{\prime}$ & $\mathrm{C} 24^{\prime}$ & $1.523(12)$ \\
\hline $\mathrm{C} 34$ & $\mathrm{C} 35$ & $1.538(11)$ & $\mathrm{C}^{\prime}$ & $\mathrm{C} 23^{\prime}$ & $1.517(11)$ \\
\hline $\mathrm{C} 34$ & $\mathrm{C} 36$ & $1.531(11)$ & $\mathrm{C} 4{ }^{\prime}$ & C3' & $1.527(11)$ \\
\hline $\mathrm{C} 35$ & $\mathrm{H} 35 \mathrm{~A}$ & 0.98 & $\mathrm{C} 24^{\prime}$ & H24D & 0.98 \\
\hline $\mathrm{C} 35$ & H35B & 0.98 & $\mathrm{C} 24^{\prime}$ & $\mathrm{H} 24 \mathrm{E}$ & 0.98 \\
\hline $\mathrm{C} 35$ & $\mathrm{H} 35 \mathrm{C}$ & 0.98 & $\mathrm{C} 24^{\prime}$ & $\mathrm{H} 24 \mathrm{~F}$ & 0.98 \\
\hline $\mathrm{C} 36$ & $\mathrm{H} 36 \mathrm{~A}$ & 0.98 & $\mathrm{C} 23^{\prime}$ & $\mathrm{H} 23 \mathrm{D}$ & 0.98 \\
\hline C36 & H36B & 0.98 & C23' & H23E & 0.98 \\
\hline
\end{tabular}




\begin{tabular}{|c|c|c|c|c|c|}
\hline $\mathrm{C} 36$ & $\mathrm{H} 36 \mathrm{C}$ & 0.98 & $\mathrm{C} 23^{\prime}$ & $\mathrm{H} 23 \mathrm{~F}$ & 0.98 \\
\hline $\mathrm{C} 4$ & $\mathrm{C} 23$ & $1.498(12)$ & C3' & H3'A & 0.99 \\
\hline $\mathrm{C} 4$ & $\mathrm{C} 24$ & $1.537(12)$ & $\mathrm{C} 3^{\prime}$ & H3'B & 0.99 \\
\hline $\mathrm{C} 4$ & $\mathrm{C} 3$ & $1.548(11)$ & C3' & $\mathrm{C} 2^{\prime}$ & $1.543(10)$ \\
\hline $\mathrm{C} 23$ & $\mathrm{H} 23 \mathrm{~A}$ & 0.98 & $\mathrm{C} 2^{\prime}$ & $\mathrm{C} 5^{\prime}$ & $1.546(11)$ \\
\hline $\mathrm{C} 23$ & $\mathrm{H} 23 \mathrm{~B}$ & 0.98 & $\mathrm{C} 2^{\prime}$ & $\mathrm{C} 22^{\prime}$ & $1.540(11)$ \\
\hline $\mathrm{C} 23$ & $\mathrm{H} 23 \mathrm{C}$ & 0.98 & $\mathrm{C} 8^{\prime}$ & H8'A & 0.98 \\
\hline $\mathrm{C} 24$ & $\mathrm{H} 24 \mathrm{~A}$ & 0.98 & $\mathrm{C} 8^{\prime}$ & H8'B & 0.98 \\
\hline $\mathrm{C} 24$ & H24B & 0.98 & C $8^{\prime}$ & $\mathrm{H} 8^{\prime} \mathrm{C}$ & 0.98 \\
\hline $\mathrm{C} 24$ & $\mathrm{H} 24 \mathrm{C}$ & 0.98 & $\mathrm{C} 8^{\prime}$ & C6' & $1.534(11)$ \\
\hline $\mathrm{C} 3$ & $\mathrm{H} 3 \mathrm{~A}$ & 0.99 & C6' & $\mathrm{C}^{\prime}$ & $1.564(11)$ \\
\hline $\mathrm{C} 3$ & $\mathrm{H} 3 \mathrm{~B}$ & 0.99 & C6' & $\mathrm{C} 7^{\prime}$ & $1.536(11)$ \\
\hline $\mathrm{C} 3$ & $\mathrm{C} 2$ & $1.551(11)$ & C6' & C9' & $1.528(11)$ \\
\hline $\mathrm{C} 2$ & $\mathrm{C} 22$ & $1.542(12)$ & $\mathrm{C} 10^{\prime}$ & $\mathrm{C} 15^{\prime}$ & $1.409(13)$ \\
\hline $\mathrm{C} 2$ & $\mathrm{C} 5$ & $1.571(12)$ & $\mathrm{C} 12^{\prime}$ & H12' & 0.95 \\
\hline $\mathrm{C} 22$ & $\mathrm{H} 22 \mathrm{~A}$ & 0.98 & $\mathrm{C} 12^{\prime}$ & $\mathrm{C} 13^{\prime}$ & $1.380(13)$ \\
\hline $\mathrm{C} 22$ & $\mathrm{H} 22 \mathrm{~B}$ & 0.98 & $\mathrm{C} 13^{\prime}$ & H13' & 0.95 \\
\hline $\mathrm{C} 22$ & $\mathrm{H} 22 \mathrm{C}$ & 0.98 & C13' & $\mathrm{C} 14^{\prime}$ & $1.388(12)$ \\
\hline $\mathrm{C} 5$ & C6 & $1.573(11)$ & C14' & H14' & 0.95 \\
\hline $\mathrm{C} 10$ & $\mathrm{C} 15$ & $1.396(12)$ & C14' & $\mathrm{C} 15^{\prime}$ & $1.382(12)$ \\
\hline $\mathrm{C} 10$ & $\mathrm{C} 11$ & $1.429(13)$ & $\mathrm{C} 21^{\prime}$ & H21D & 0.98 \\
\hline $\mathrm{C} 15$ & $\mathrm{C} 14$ & $1.391(12)$ & $\mathrm{C} 21^{\prime}$ & $\mathrm{H} 21 \mathrm{E}$ & 0.98 \\
\hline $\mathrm{C} 15$ & C19 & $1.547(12)$ & $\mathrm{C} 21^{\prime}$ & $\mathrm{H} 21 \mathrm{~F}$ & 0.98 \\
\hline $\mathrm{C} 14$ & H14 & 0.95 & $\mathrm{C} 20^{\prime}$ & H20D & 0.98 \\
\hline $\mathrm{C} 14$ & $\mathrm{C} 13$ & $1.365(12)$ & $\mathrm{C} 20^{\prime}$ & $\mathrm{H} 20 \mathrm{E}$ & 0.98 \\
\hline $\mathrm{C} 13$ & H13 & 0.95 & $\mathrm{C} 20^{\prime}$ & $\mathrm{H} 20 \mathrm{~F}$ & 0.98 \\
\hline $\mathrm{C} 13$ & $\mathrm{C} 12$ & $1.367(13)$ & C16' & H16' & 1 \\
\hline $\mathrm{C} 12$ & H12 & 0.95 & $\mathrm{C} 16^{\prime}$ & $\mathrm{C} 17^{\prime}$ & $1.528(11)$ \\
\hline $\mathrm{C} 12$ & $\mathrm{C} 11$ & $1.378(13)$ & C16' & $\mathrm{C} 18^{\prime}$ & $1.551(12)$ \\
\hline $\mathrm{C} 11$ & $\mathrm{C} 16$ & $1.523(12)$ & $\mathrm{C} 17^{\prime}$ & H17D & 0.98 \\
\hline C16 & H16 & 1 & $\mathrm{C} 17^{\prime}$ & $\mathrm{H} 17 \mathrm{E}$ & 0.98 \\
\hline $\mathrm{C} 16$ & $\mathrm{C} 17$ & $1.529(12)$ & $\mathrm{C} 17^{\prime}$ & $\mathrm{H} 17 \mathrm{~F}$ & 0.98 \\
\hline $\mathrm{C} 16$ & $\mathrm{C} 18$ & $1.519(11)$ & $\mathrm{C} 18^{\prime}$ & $\mathrm{H} 18 \mathrm{D}$ & 0.98 \\
\hline C17 & H17A & 0.98 & $\mathrm{C} 18^{\prime}$ & H18E & 0.98 \\
\hline $\mathrm{C} 17$ & H17B & 0.98 & $\mathrm{C} 18^{\prime}$ & $\mathrm{H} 18 \mathrm{~F}$ & 0.98 \\
\hline $\mathrm{C} 17$ & $\mathrm{H} 17 \mathrm{C}$ & 0.98 & $\mathrm{C} 7^{\prime}$ & H7'A & 0.98 \\
\hline $\mathrm{C} 18$ & H18A & 0.98 & $C 7^{\prime}$ & H7'B & 0.98 \\
\hline $\mathrm{C} 18$ & H18B & 0.98 & C7' & $\mathrm{H} 7^{\prime} \mathrm{C}$ & 0.98 \\
\hline $\mathrm{C} 18$ & $\mathrm{H} 18 \mathrm{C}$ & 0.98 & C9' & H9'A & 0.98 \\
\hline C19 & H19 & 1 & C9' & H9'B & 0.98 \\
\hline C19 & $\mathrm{C} 21$ & $1.517(12)$ & C9' & $\mathrm{H}^{\prime}{ }^{\prime} \mathrm{C}$ & 0.98 \\
\hline C19 & $\mathrm{C} 20$ & $1.537(12)$ & $\mathrm{C} 22^{\prime}$ & $\mathrm{H} 22 \mathrm{D}$ & 0.98 \\
\hline $\mathrm{C} 21$ & $\mathrm{H} 21 \mathrm{~A}$ & 0.98 & $\mathrm{C} 22^{\prime}$ & $\mathrm{H} 22 \mathrm{E}$ & 0.98 \\
\hline $\mathrm{C} 21$ & H21B & 0.98 & C22' & $\mathrm{H} 22 \mathrm{~F}$ & 0.98 \\
\hline
\end{tabular}


Table S6.2.3. Bond angles for (6e)AuCl.

\begin{tabular}{|c|c|c|c|c|c|c|c|}
\hline Atom & Atom & Atom & Angle $/^{\circ}$ & Atom & Atom & Atom & Angle $/^{\circ}$ \\
\hline $\mathrm{C} 1$ & Au1 & $\mathrm{Cl1}$ & 176.4(3) & $\mathrm{C} 6$ & $\mathrm{C} 7$ & H7B & 109.5 \\
\hline $\mathrm{C} 1^{\prime}$ & $\mathrm{Au} 1^{\prime}$ & Cl1' & 176.6(3) & C6 & $\mathrm{C} 7$ & $\mathrm{H} 7 \mathrm{C}$ & 109.5 \\
\hline $\mathrm{C} 1$ & N1 & $\mathrm{C} 25$ & $123.2(7)$ & $\mathrm{H} 7 \mathrm{~A}$ & $\mathrm{C} 7$ & $\mathrm{H} 7 \mathrm{~B}$ & 109.5 \\
\hline $\mathrm{C} 1$ & N1 & $\mathrm{C} 4$ & $115.9(7)$ & $\mathrm{H} 7 \mathrm{~A}$ & $\mathrm{C} 7$ & $\mathrm{H} 7 \mathrm{C}$ & 109.5 \\
\hline $\mathrm{C} 25$ & N1 & $\mathrm{C} 4$ & $120.9(7)$ & H7B & $\mathrm{C} 7$ & $\mathrm{H} 7 \mathrm{C}$ & 109.5 \\
\hline $\mathrm{C} 5$ & N2 & $\mathrm{C} 10$ & 129.7(8) & $\mathrm{C} 6$ & C9 & H9A & 109.5 \\
\hline $\mathrm{C} 25^{\prime}$ & N1' & $\mathrm{C} 4{ }^{\prime}$ & $120.0(6)$ & $\mathrm{C} 6$ & C9 & H9B & 109.5 \\
\hline $\mathrm{C} 1^{\prime}$ & $\mathrm{N} 1^{\prime}$ & $\mathrm{C} 25^{\prime}$ & $124.4(7)$ & $\mathrm{C} 6$ & C9 & $\mathrm{H} 9 \mathrm{C}$ & 109.5 \\
\hline $\mathrm{C} 1^{\prime}$ & N1' & $\mathrm{C} 4{ }^{\prime}$ & 115.6(7) & H9A & C9 & H9B & 109.5 \\
\hline $\mathrm{C} 30^{\prime}$ & $\mathrm{C} 25^{\prime}$ & $\mathrm{N} 1{ }^{\prime}$ & $120.8(8)$ & H9A & C9 & $\mathrm{H} 9 \mathrm{C}$ & 109.5 \\
\hline $\mathrm{C} 30^{\prime}$ & $\mathrm{C} 25^{\prime}$ & $\mathrm{C} 26^{\prime}$ & 121.9(8) & H9B & C9 & H9C & 109.5 \\
\hline $\mathrm{C} 26^{\prime}$ & $\mathrm{C} 25^{\prime}$ & N1' & $117.3(8)$ & C6 & $\mathrm{C} 8$ & $\mathrm{H} 8 \mathrm{~A}$ & 109.5 \\
\hline $\mathrm{C} 5^{\prime}$ & N2' & $\mathrm{C} 10^{\prime}$ & 131.0(8) & $\mathrm{C} 6$ & $\mathrm{C} 8$ & $\mathrm{H} 8 \mathrm{~B}$ & 109.5 \\
\hline $\mathrm{C} 10^{\prime}$ & $\mathrm{C} 11^{\prime}$ & C16' & $120.2(8)$ & $\mathrm{C} 6$ & $\mathrm{C} 8$ & $\mathrm{H} 8 \mathrm{C}$ & 109.5 \\
\hline $\mathrm{C} 12^{\prime}$ & $\mathrm{C} 11^{\prime}$ & $\mathrm{C} 10^{\prime}$ & 118.5(9) & H8A & $\mathrm{C} 8$ & $\mathrm{H} 8 \mathrm{~B}$ & 109.5 \\
\hline $\mathrm{C} 12^{\prime}$ & $\mathrm{C} 11^{\prime}$ & C16' & 121.3(9) & H8A & $\mathrm{C} 8$ & $\mathrm{H} 8 \mathrm{C}$ & 109.5 \\
\hline $\mathrm{C} 15^{\prime}$ & $\mathrm{C} 19^{\prime}$ & H19' & 107.4 & $\mathrm{H} 8 \mathrm{~B}$ & $\mathrm{C} 8$ & $\mathrm{H} 8 \mathrm{C}$ & 109.5 \\
\hline $\mathrm{C} 15^{\prime}$ & $\mathrm{C} 19^{\prime}$ & $\mathrm{C} 21^{\prime}$ & $113.2(8)$ & N1' & $\mathrm{C} 1^{\prime}$ & Aul' & $125.7(7)$ \\
\hline $\mathrm{C} 15^{\prime}$ & $\mathrm{C} 19^{\prime}$ & $\mathrm{C} 20^{\prime}$ & $112.2(8)$ & N1' & $\mathrm{C} 1{ }^{\prime}$ & $\mathrm{C} 2^{\prime}$ & $110.4(7)$ \\
\hline $\mathrm{C} 21^{\prime}$ & $\mathrm{C} 19^{\prime}$ & H19' & 107.4 & $\mathrm{C} 2^{\prime}$ & $\mathrm{C} 1^{\prime}$ & Aul' & $123.2(6)$ \\
\hline $\mathrm{C} 21^{\prime}$ & $\mathrm{C} 19^{\prime}$ & $\mathrm{C} 20^{\prime}$ & $109.0(7)$ & $\mathrm{C} 25^{\prime}$ & $\mathrm{C} 30^{\prime}$ & C34' & 124.1(8) \\
\hline $\mathrm{C} 20^{\prime}$ & $\mathrm{C} 19^{\prime}$ & H19' & 107.4 & C29' & $\mathrm{C} 30^{\prime}$ & $\mathrm{C} 25^{\prime}$ & $116.8(8)$ \\
\hline N1 & $\mathrm{C} 1$ & Au1 & $127.3(7)$ & C29' & C30' & C34' & $118.8(8)$ \\
\hline N1 & $\mathrm{C} 1$ & $\mathrm{C} 2$ & $110.2(7)$ & C $30^{\prime}$ & C34' & H34' & 108.2 \\
\hline $\mathrm{C} 2$ & $\mathrm{C} 1$ & $\mathrm{Au} 1$ & $121.9(6)$ & $\mathrm{C} 30^{\prime}$ & C34' & $\mathrm{C} 36^{\prime}$ & $113.4(7)$ \\
\hline $\mathrm{C} 26$ & $\mathrm{C} 25$ & N1 & $117.3(8)$ & $\mathrm{C} 30^{\prime}$ & C34' & $\mathrm{C} 35^{\prime}$ & 108.9(7) \\
\hline $\mathrm{C} 26$ & $\mathrm{C} 25$ & $\mathrm{C} 30$ & $123.5(8)$ & $\mathrm{C} 36^{\prime}$ & C34' & H34' & 108.2 \\
\hline $\mathrm{C} 30$ & $\mathrm{C} 25$ & N1 & $119.2(8)$ & C35' & C34' & H34' & 108.2 \\
\hline $\mathrm{C} 25$ & $\mathrm{C} 26$ & $\mathrm{C} 31$ & $123.5(8)$ & C35' & C34' & C36' & $109.8(7)$ \\
\hline $\mathrm{C} 25$ & $\mathrm{C} 26$ & $\mathrm{C} 27$ & 116.2(9) & C34' & C36' & $\mathrm{H} 36 \mathrm{D}$ & 109.5 \\
\hline $\mathrm{C} 27$ & $\mathrm{C} 26$ & $\mathrm{C} 31$ & $120.0(9)$ & C34' & C36' & $\mathrm{H} 36 \mathrm{E}$ & 109.5 \\
\hline $\mathrm{C} 26$ & C31 & $\mathrm{H} 31$ & 108.1 & C34' & C36' & $\mathrm{H} 36 \mathrm{~F}$ & 109.5 \\
\hline $\mathrm{C} 26$ & C31 & $\mathrm{C} 33$ & $112.7(7)$ & H36D & C36' & $\mathrm{H} 36 \mathrm{E}$ & 109.5 \\
\hline $\mathrm{C} 26$ & $\mathrm{C} 31$ & $\mathrm{C} 32$ & $111.0(7)$ & $\mathrm{H} 36 \mathrm{D}$ & C36' & $\mathrm{H} 36 \mathrm{~F}$ & 109.5 \\
\hline $\mathrm{C} 33$ & $\mathrm{C} 31$ & H31 & 108.1 & $\mathrm{H} 36 \mathrm{E}$ & C36' & $\mathrm{H} 36 \mathrm{~F}$ & 109.5 \\
\hline C32 & C31 & H31 & 108.1 & C34' & C35' & H35D & 109.5 \\
\hline $\mathrm{C} 32$ & $\mathrm{C} 31$ & $\mathrm{C} 33$ & $108.7(8)$ & C34' & C35' & $\mathrm{H} 35 \mathrm{E}$ & 109.5 \\
\hline $\mathrm{C} 31$ & $\mathrm{C} 33$ & $\mathrm{H} 33 \mathrm{~A}$ & 109.5 & C34' & C35' & $\mathrm{H} 35 \mathrm{~F}$ & 109.5 \\
\hline $\mathrm{C} 31$ & $\mathrm{C} 33$ & H33B & 109.5 & $\mathrm{H} 35 \mathrm{D}$ & C35' & H35E & 109.5 \\
\hline $\mathrm{C} 31$ & $\mathrm{C} 33$ & $\mathrm{H} 33 \mathrm{C}$ & 109.5 & $\mathrm{H} 35 \mathrm{D}$ & C35' & $\mathrm{H} 35 \mathrm{~F}$ & 109.5 \\
\hline
\end{tabular}




\begin{tabular}{|c|c|c|c|c|c|c|c|}
\hline $\mathrm{H} 33 \mathrm{~A}$ & $\mathrm{C} 33$ & H33B & 109.5 & H35E & $\mathrm{C} 35^{\prime}$ & $\mathrm{H} 35 \mathrm{~F}$ & 109.5 \\
\hline $\mathrm{H} 33 \mathrm{~A}$ & $\mathrm{C} 33$ & $\mathrm{H} 33 \mathrm{C}$ & 109.5 & $\mathrm{C} 30^{\prime}$ & $\mathrm{C} 29^{\prime}$ & $\mathrm{H} 29^{\prime}$ & 118.9 \\
\hline H33B & $\mathrm{C} 33$ & $\mathrm{H} 33 \mathrm{C}$ & 109.5 & $\mathrm{C} 28^{\prime}$ & $\mathrm{C} 29^{\prime}$ & $\mathrm{C} 30^{\prime}$ & $122.2(9)$ \\
\hline $\mathrm{C} 31$ & $\mathrm{C} 32$ & $\mathrm{H} 32 \mathrm{~A}$ & 109.5 & $\mathrm{C} 28^{\prime}$ & $\mathrm{C} 29^{\prime}$ & $\mathrm{H} 29^{\prime}$ & 118.9 \\
\hline $\mathrm{C} 31$ & $\mathrm{C} 32$ & H32B & 109.5 & $\mathrm{C} 29^{\prime}$ & $\mathrm{C} 28^{\prime}$ & $\mathrm{H} 28^{\prime}$ & 119.8 \\
\hline $\mathrm{C} 31$ & $\mathrm{C} 32$ & $\mathrm{H} 32 \mathrm{C}$ & 109.5 & $\mathrm{C} 29^{\prime}$ & $\mathrm{C} 28^{\prime}$ & $\mathrm{C} 27^{\prime}$ & $120.5(8)$ \\
\hline $\mathrm{H} 32 \mathrm{~A}$ & $\mathrm{C} 32$ & H32B & 109.5 & $\mathrm{C} 27^{\prime}$ & $\mathrm{C} 28^{\prime}$ & $\mathrm{H} 28^{\prime}$ & 119.8 \\
\hline $\mathrm{H} 32 \mathrm{~A}$ & $\mathrm{C} 32$ & $\mathrm{H} 32 \mathrm{C}$ & 109.5 & C28' & $\mathrm{C} 27^{\prime}$ & $\mathrm{H} 27^{\prime}$ & 119.6 \\
\hline H32B & $\mathrm{C} 32$ & $\mathrm{H} 32 \mathrm{C}$ & 109.5 & $\mathrm{C} 28^{\prime}$ & $\mathrm{C} 27^{\prime}$ & $\mathrm{C} 26^{\prime}$ & $120.8(8)$ \\
\hline $\mathrm{C} 26$ & $\mathrm{C} 27$ & $\mathrm{H} 27$ & 118.9 & C26' & $\mathrm{C} 27^{\prime}$ & $\mathrm{H} 27^{\prime}$ & 119.6 \\
\hline $\mathrm{C} 28$ & $\mathrm{C} 27$ & $\mathrm{C} 26$ & $122.1(10)$ & $\mathrm{C} 25^{\prime}$ & $\mathrm{C} 26^{\prime}$ & $\mathrm{C} 31^{\prime}$ & $122.3(8)$ \\
\hline $\mathrm{C} 28$ & $\mathrm{C} 27$ & $\mathrm{H} 27$ & 118.9 & $\mathrm{C} 27^{\prime}$ & $\mathrm{C} 26^{\prime}$ & $\mathrm{C} 25^{\prime}$ & $117.6(8)$ \\
\hline $\mathrm{C} 27$ & $\mathrm{C} 28$ & $\mathrm{H} 28$ & 119.5 & $\mathrm{C} 27^{\prime}$ & $\mathrm{C} 26^{\prime}$ & C31' & $120.1(9)$ \\
\hline $\mathrm{C} 27$ & $\mathrm{C} 28$ & $\mathrm{C} 29$ & $121.0(9)$ & $\mathrm{C} 26^{\prime}$ & $\mathrm{C} 31^{\prime}$ & H31' & 107.1 \\
\hline $\mathrm{C} 29$ & $\mathrm{C} 28$ & $\mathrm{H} 28$ & 119.5 & C33' & $\mathrm{C} 31^{\prime}$ & $\mathrm{C} 26^{\prime}$ & $113.9(7)$ \\
\hline $\mathrm{C} 28$ & $\mathrm{C} 29$ & $\mathrm{H} 29$ & 119.8 & C33' & $\mathrm{C} 31^{\prime}$ & H31' & 107.1 \\
\hline $\mathrm{C} 28$ & $\mathrm{C} 29$ & $\mathrm{C} 30$ & $120.3(10)$ & C33' & $\mathrm{C} 31^{\prime}$ & $\mathrm{C} 32^{\prime}$ & $111.8(7)$ \\
\hline $\mathrm{C} 30$ & $\mathrm{C} 29$ & $\mathrm{H} 29$ & 119.8 & C32' & $\mathrm{C} 31^{\prime}$ & $\mathrm{C} 26^{\prime}$ & $109.5(7)$ \\
\hline $\mathrm{C} 25$ & $\mathrm{C} 30$ & $\mathrm{C} 34$ & $125.0(8)$ & C32' & $\mathrm{C} 31^{\prime}$ & H31' & 107.1 \\
\hline $\mathrm{C} 29$ & $\mathrm{C} 30$ & $\mathrm{C} 25$ & $116.7(9)$ & $\mathrm{C} 31^{\prime}$ & C33' & H33D & 109.5 \\
\hline $\mathrm{C} 29$ & $\mathrm{C} 30$ & $\mathrm{C} 34$ & $118.0(9)$ & C31' & C33' & $\mathrm{H} 33 \mathrm{E}$ & 109.5 \\
\hline $\mathrm{C} 30$ & $\mathrm{C} 34$ & $\mathrm{H} 34$ & 108.4 & C31' & C33' & $\mathrm{H} 33 \mathrm{~F}$ & 109.5 \\
\hline $\mathrm{C} 30$ & $\mathrm{C} 34$ & $\mathrm{C} 35$ & $108.2(7)$ & $\mathrm{H} 33 \mathrm{D}$ & $\mathrm{C} 33^{\prime}$ & H33E & 109.5 \\
\hline $\mathrm{C} 30$ & $\mathrm{C} 34$ & C36 & $114.1(7)$ & H33D & C33' & $\mathrm{H} 33 \mathrm{~F}$ & 109.5 \\
\hline C35 & $\mathrm{C} 34$ & $\mathrm{H} 34$ & 108.4 & $\mathrm{H} 33 \mathrm{E}$ & C33' & $\mathrm{H} 33 \mathrm{~F}$ & 109.5 \\
\hline $\mathrm{C} 36$ & $\mathrm{C} 34$ & H34 & 108.4 & C31' & $\mathrm{C} 32^{\prime}$ & $\mathrm{H} 32 \mathrm{D}$ & 109.5 \\
\hline $\mathrm{C} 36$ & $\mathrm{C} 34$ & $\mathrm{C} 35$ & $109.2(8)$ & C31' & $\mathrm{C} 32^{\prime}$ & $\mathrm{H} 32 \mathrm{E}$ & 109.5 \\
\hline $\mathrm{C} 34$ & $\mathrm{C} 35$ & $\mathrm{H} 35 \mathrm{~A}$ & 109.5 & C31' & $\mathrm{C} 32^{\prime}$ & $\mathrm{H} 32 \mathrm{~F}$ & 109.5 \\
\hline $\mathrm{C} 34$ & $\mathrm{C} 35$ & H35B & 109.5 & $\mathrm{H} 32 \mathrm{D}$ & $\mathrm{C} 32^{\prime}$ & $\mathrm{H} 32 \mathrm{E}$ & 109.5 \\
\hline $\mathrm{C} 34$ & $\mathrm{C} 35$ & $\mathrm{H} 35 \mathrm{C}$ & 109.5 & $\mathrm{H} 32 \mathrm{D}$ & $\mathrm{C} 32^{\prime}$ & $\mathrm{H} 32 \mathrm{~F}$ & 109.5 \\
\hline $\mathrm{H} 35 \mathrm{~A}$ & $\mathrm{C} 35$ & $\mathrm{H} 35 \mathrm{~B}$ & 109.5 & $\mathrm{H} 32 \mathrm{E}$ & $\mathrm{C} 32^{\prime}$ & $\mathrm{H} 32 \mathrm{~F}$ & 109.5 \\
\hline $\mathrm{H} 35 \mathrm{~A}$ & $\mathrm{C} 35$ & $\mathrm{H} 35 \mathrm{C}$ & 109.5 & C24' & $\mathrm{C} 4^{\prime}$ & N1' & 109.1(7) \\
\hline H35B & $\mathrm{C} 35$ & $\mathrm{H} 35 \mathrm{C}$ & 109.5 & $\mathrm{C} 24^{\prime}$ & $\mathrm{C}^{\prime}$ & $\mathrm{C} 3^{\prime}$ & $113.5(8)$ \\
\hline $\mathrm{C} 34$ & $\mathrm{C} 36$ & $\mathrm{H} 36 \mathrm{~A}$ & 109.5 & C23' & $\mathrm{C} 4^{\prime}$ & N1' & $112.6(7)$ \\
\hline C34 & $\mathrm{C} 36$ & H36B & 109.5 & C23' & $\mathrm{C}^{\prime}$ & $\mathrm{C} 24^{\prime}$ & $108.4(7)$ \\
\hline $\mathrm{C} 34$ & $\mathrm{C} 36$ & $\mathrm{H} 36 \mathrm{C}$ & 109.5 & C23' & $\mathrm{C} 4^{\prime}$ & C3' & $113.5(8)$ \\
\hline $\mathrm{H} 36 \mathrm{~A}$ & $\mathrm{C} 36$ & H36B & 109.5 & $\mathrm{C} 3^{\prime}$ & $\mathrm{C}^{\prime}$ & N1' & $99.6(6)$ \\
\hline $\mathrm{H} 36 \mathrm{~A}$ & $\mathrm{C} 36$ & $\mathrm{H} 36 \mathrm{C}$ & 109.5 & C4' & $\mathrm{C} 24^{\prime}$ & $\mathrm{H} 24 \mathrm{D}$ & 109.5 \\
\hline H36B & $\mathrm{C} 36$ & $\mathrm{H} 36 \mathrm{C}$ & 109.5 & $\mathrm{C} 4^{\prime}$ & $\mathrm{C} 24^{\prime}$ & $\mathrm{H} 24 \mathrm{E}$ & 109.5 \\
\hline N1 & $\mathrm{C} 4$ & $\mathrm{C} 24$ & $109.0(7)$ & $\mathrm{C} 4^{\prime}$ & $\mathrm{C} 24^{\prime}$ & $\mathrm{H} 24 \mathrm{~F}$ & 109.5 \\
\hline $\mathrm{N} 1$ & $\mathrm{C} 4$ & $\mathrm{C} 3$ & $101.4(6)$ & $\mathrm{H} 24 \mathrm{D}$ & $\mathrm{C} 24^{\prime}$ & $\mathrm{H} 24 \mathrm{E}$ & 109.5 \\
\hline $\mathrm{C} 23$ & $\mathrm{C} 4$ & N1 & $111.8(8)$ & $\mathrm{H} 24 \mathrm{D}$ & $\mathrm{C} 24^{\prime}$ & $\mathrm{H} 24 \mathrm{~F}$ & 109.5 \\
\hline $\mathrm{C} 23$ & $\mathrm{C} 4$ & $\mathrm{C} 24$ & $109.7(7)$ & $\mathrm{H} 24 \mathrm{E}$ & $\mathrm{C} 24^{\prime}$ & $\mathrm{H} 24 \mathrm{~F}$ & 109.5 \\
\hline $\mathrm{C} 23$ & $\mathrm{C} 4$ & $\mathrm{C} 3$ & $111.8(8)$ & C4' & C23' & $\mathrm{H} 23 \mathrm{D}$ & 109.5 \\
\hline
\end{tabular}




\begin{tabular}{|c|c|c|c|c|c|c|c|}
\hline $\mathrm{C} 24$ & $\mathrm{C} 4$ & $\mathrm{C} 3$ & $112.8(8)$ & $\mathrm{C} 4^{\prime}$ & $\mathrm{C} 23^{\prime}$ & $\mathrm{H} 23 \mathrm{E}$ & 109.5 \\
\hline $\mathrm{C} 4$ & $\mathrm{C} 23$ & $\mathrm{H} 23 \mathrm{~A}$ & 109.5 & $\mathrm{C} 4^{\prime}$ & $\mathrm{C} 23^{\prime}$ & $\mathrm{H} 23 \mathrm{~F}$ & 109.5 \\
\hline $\mathrm{C} 4$ & $\mathrm{C} 23$ & H23B & 109.5 & $\mathrm{H} 23 \mathrm{D}$ & $\mathrm{C} 23^{\prime}$ & $\mathrm{H} 23 \mathrm{E}$ & 109.5 \\
\hline $\mathrm{C} 4$ & $\mathrm{C} 23$ & $\mathrm{H} 23 \mathrm{C}$ & 109.5 & $\mathrm{H} 23 \mathrm{D}$ & $\mathrm{C} 23^{\prime}$ & $\mathrm{H} 23 \mathrm{~F}$ & 109.5 \\
\hline $\mathrm{H} 23 \mathrm{~A}$ & $\mathrm{C} 23$ & H23B & 109.5 & $\mathrm{H} 23 \mathrm{E}$ & $\mathrm{C} 23^{\prime}$ & $\mathrm{H} 23 \mathrm{~F}$ & 109.5 \\
\hline $\mathrm{H} 23 \mathrm{~A}$ & $\mathrm{C} 23$ & $\mathrm{H} 23 \mathrm{C}$ & 109.5 & $\mathrm{C} 4{ }^{\prime}$ & C3' & H3'A & 109.9 \\
\hline $\mathrm{H} 23 \mathrm{~B}$ & $\mathrm{C} 23$ & $\mathrm{H} 23 \mathrm{C}$ & 109.5 & $\mathrm{C} 4^{\prime}$ & C3' & H3'B & 109.9 \\
\hline $\mathrm{C} 4$ & $\mathrm{C} 24$ & $\mathrm{H} 24 \mathrm{~A}$ & 109.5 & $\mathrm{C} 4^{\prime}$ & C3' & $\mathrm{C} 2^{\prime}$ & 109.1(7) \\
\hline $\mathrm{C} 4$ & $\mathrm{C} 24$ & H24B & 109.5 & H3'A & $\mathrm{C} 3^{\prime}$ & H3'B & 108.3 \\
\hline $\mathrm{C} 4$ & $\mathrm{C} 24$ & $\mathrm{H} 24 \mathrm{C}$ & 109.5 & $\mathrm{C} 2^{\prime}$ & $\mathrm{C} 3^{\prime}$ & $\mathrm{H}^{\prime} \mathrm{A}$ & 109.9 \\
\hline $\mathrm{H} 24 \mathrm{~A}$ & $\mathrm{C} 24$ & H24B & 109.5 & $\mathrm{C} 2^{\prime}$ & C3' & H3'B & 109.9 \\
\hline $\mathrm{H} 24 \mathrm{~A}$ & $\mathrm{C} 24$ & $\mathrm{H} 24 \mathrm{C}$ & 109.5 & $\mathrm{C} 1^{\prime}$ & $\mathrm{C} 2^{\prime}$ & C3' & $102.5(6)$ \\
\hline $\mathrm{H} 24 \mathrm{~B}$ & $\mathrm{C} 24$ & $\mathrm{H} 24 \mathrm{C}$ & 109.5 & $\mathrm{C} 1^{\prime}$ & $\mathrm{C} 2^{\prime}$ & C5' & $109.5(7)$ \\
\hline $\mathrm{C} 4$ & $\mathrm{C} 3$ & H3A & 110.4 & $\mathrm{C} 1{ }^{\prime}$ & $\mathrm{C} 2^{\prime}$ & $\mathrm{C} 22^{\prime}$ & $102.9(7)$ \\
\hline $\mathrm{C} 4$ & $\mathrm{C} 3$ & H3B & 110.4 & C3' & $\mathrm{C} 2^{\prime}$ & $\mathrm{C}^{\prime}$ & $110.5(7)$ \\
\hline $\mathrm{C} 4$ & $\mathrm{C} 3$ & $\mathrm{C} 2$ & $106.5(7)$ & $\mathrm{C} 22^{\prime}$ & $\mathrm{C} 2^{\prime}$ & C3' & $110.6(7)$ \\
\hline $\mathrm{H} 3 \mathrm{~A}$ & $\mathrm{C} 3$ & H3B & 108.6 & $\mathrm{C} 22^{\prime}$ & $\mathrm{C} 2^{\prime}$ & $\mathrm{C}^{\prime}$ & $119.3(7)$ \\
\hline $\mathrm{C} 2$ & $\mathrm{C} 3$ & $\mathrm{H} 3 \mathrm{~A}$ & 110.4 & H8'A & $\mathrm{C} 8^{\prime}$ & $\mathrm{H} 8^{\prime} \mathrm{B}$ & 109.5 \\
\hline $\mathrm{C} 2$ & $\mathrm{C} 3$ & $\mathrm{H} 3 \mathrm{~B}$ & 110.4 & H8'A & $\mathrm{C} 8^{\prime}$ & $\mathrm{H} 8^{\prime} \mathrm{C}$ & 109.5 \\
\hline $\mathrm{C} 1$ & $\mathrm{C} 2$ & $\mathrm{C} 3$ & $104.0(7)$ & H8'B & C $8^{\prime}$ & $\mathrm{H}^{\prime} \mathrm{C}$ & 109.5 \\
\hline $\mathrm{C} 1$ & $\mathrm{C} 2$ & $\mathrm{C} 22$ & $104.9(7)$ & $\mathrm{C} 6^{\prime}$ & $\mathrm{C} 8^{\prime}$ & H8'A & 109.5 \\
\hline $\mathrm{C} 1$ & $\mathrm{C} 2$ & $\mathrm{C} 5$ & 106.6(7) & $\mathrm{C} 6^{\prime}$ & C $8^{\prime}$ & $\mathrm{H}^{\prime} \mathrm{B}$ & 109.5 \\
\hline $\mathrm{C} 3$ & $\mathrm{C} 2$ & $\mathrm{C} 5$ & $108.7(7)$ & C6' & C8' & $\mathrm{H}^{\prime} \mathrm{C}$ & 109.5 \\
\hline $\mathrm{C} 22$ & $\mathrm{C} 2$ & $\mathrm{C} 3$ & $112.3(8)$ & $\mathrm{C} 8^{\prime}$ & C6' & $\mathrm{C}^{\prime}$ & 109.4(7) \\
\hline $\mathrm{C} 22$ & $\mathrm{C} 2$ & $\mathrm{C} 5$ & $119.1(7)$ & $\mathrm{C} 8^{\prime}$ & C6' & C7' & $109.9(7)$ \\
\hline $\mathrm{C} 2$ & $\mathrm{C} 22$ & $\mathrm{H} 22 \mathrm{~A}$ & 109.5 & $\mathrm{C} 7^{\prime}$ & C6' & $\mathrm{C}^{\prime}$ & $109.9(7)$ \\
\hline $\mathrm{C} 2$ & $\mathrm{C} 22$ & $\mathrm{H} 22 \mathrm{~B}$ & 109.5 & $\mathrm{C}^{\prime}$ & $\mathrm{C} 6^{\prime}$ & $\mathrm{C} 8^{\prime}$ & $106.7(7)$ \\
\hline $\mathrm{C} 2$ & $\mathrm{C} 22$ & $\mathrm{H} 22 \mathrm{C}$ & 109.5 & C9' & C6' & $\mathrm{C}^{\prime}$ & $113.9(7)$ \\
\hline $\mathrm{H} 22 \mathrm{~A}$ & $\mathrm{C} 22$ & H22B & 109.5 & C9' & C6' & C7' & $106.8(7)$ \\
\hline $\mathrm{H} 22 \mathrm{~A}$ & $\mathrm{C} 22$ & $\mathrm{H} 22 \mathrm{C}$ & 109.5 & $\mathrm{~N} 2{ }^{\prime}$ & $\mathrm{C} 5^{\prime}$ & $\mathrm{C} 2{ }^{\prime}$ & $112.4(7)$ \\
\hline $\mathrm{H} 22 \mathrm{~B}$ & $\mathrm{C} 22$ & $\mathrm{H} 22 \mathrm{C}$ & 109.5 & $\mathrm{~N} 2^{\prime}$ & C5' & C6' & $128.2(8)$ \\
\hline $\mathrm{N} 2$ & $\mathrm{C} 5$ & $\mathrm{C} 2$ & $112.0(7)$ & $\mathrm{C} 2^{\prime}$ & C5' & C6' & $119.2(7)$ \\
\hline $\mathrm{N} 2$ & $\mathrm{C} 5$ & C6 & $128.5(8)$ & C11' & $\mathrm{C} 10^{\prime}$ & $\mathrm{N} 2^{\prime}$ & $120.2(9)$ \\
\hline $\mathrm{C} 2$ & $\mathrm{C} 5$ & C6 & $119.5(7)$ & C11' & $\mathrm{C} 10^{\prime}$ & $\mathrm{C} 15^{\prime}$ & $120.2(8)$ \\
\hline $\mathrm{N} 2$ & $\mathrm{C} 10$ & $\mathrm{C} 11$ & 119.3(9) & $\mathrm{C} 15^{\prime}$ & $\mathrm{C} 10^{\prime}$ & N2' & $119.2(8)$ \\
\hline $\mathrm{C} 15$ & $\mathrm{C} 10$ & $\mathrm{~N} 2$ & $120.9(9)$ & C11' & $\mathrm{C} 12^{\prime}$ & H12' & 119.3 \\
\hline $\mathrm{C} 15$ & $\mathrm{C} 10$ & $\mathrm{C} 11$ & 119.4(9) & C13' & $\mathrm{C} 12^{\prime}$ & $\mathrm{C} 11^{\prime}$ & 121.4(9) \\
\hline $\mathrm{C} 10$ & $\mathrm{C} 15$ & C19 & $121.6(9)$ & C13' & $\mathrm{C} 12^{\prime}$ & H12' & 119.3 \\
\hline $\mathrm{C} 14$ & $\mathrm{C} 15$ & $\mathrm{C} 10$ & 119.9(9) & $\mathrm{C} 12^{\prime}$ & $\mathrm{C} 13^{\prime}$ & H13' & 120 \\
\hline $\mathrm{C} 14$ & $\mathrm{C} 15$ & $\mathrm{C} 19$ & $118.5(9)$ & $\mathrm{C} 12^{\prime}$ & $\mathrm{C} 13^{\prime}$ & $\mathrm{C} 14^{\prime}$ & $119.9(9)$ \\
\hline $\mathrm{C} 15$ & $\mathrm{C} 14$ & H14 & 119.7 & C14' & $\mathrm{C} 13^{\prime}$ & H13' & 120 \\
\hline $\mathrm{C} 13$ & $\mathrm{C} 14$ & $\mathrm{C} 15$ & $120.6(9)$ & C13' & $\mathrm{C} 14^{\prime}$ & H14' & 119.7 \\
\hline $\mathrm{C} 13$ & $\mathrm{C} 14$ & H14 & 119.7 & C15' & $\mathrm{C} 14^{\prime}$ & $\mathrm{C} 13^{\prime}$ & $120.5(9)$ \\
\hline $\mathrm{C} 14$ & $\mathrm{C} 13$ & H13 & 120.2 & C15' & C14' & H14' & 119.7 \\
\hline
\end{tabular}




\begin{tabular}{|c|c|c|c|c|c|c|c|}
\hline $\mathrm{C} 14$ & $\mathrm{C} 13$ & $\mathrm{C} 12$ & 119.6(9) & $\mathrm{C} 10^{\prime}$ & $\mathrm{C} 15^{\prime}$ & $\mathrm{C} 19^{\prime}$ & $122.3(8)$ \\
\hline $\mathrm{C} 12$ & $\mathrm{C} 13$ & $\mathrm{H} 13$ & 120.2 & C14' & $\mathrm{C} 15^{\prime}$ & $\mathrm{C} 19^{\prime}$ & $118.3(9)$ \\
\hline $\mathrm{C} 13$ & $\mathrm{C} 12$ & H12 & 118.5 & C14' & $\mathrm{C} 15^{\prime}$ & $\mathrm{C} 10^{\prime}$ & $119.4(9)$ \\
\hline $\mathrm{C} 13$ & $\mathrm{C} 12$ & $\mathrm{C} 11$ & $122.9(9)$ & C19' & $\mathrm{C} 21^{\prime}$ & $\mathrm{H} 21 \mathrm{D}$ & 109.5 \\
\hline $\mathrm{C} 11$ & $\mathrm{C} 12$ & H12 & 118.5 & C19' & $\mathrm{C} 21^{\prime}$ & $\mathrm{H} 21 \mathrm{E}$ & 109.5 \\
\hline $\mathrm{C} 10$ & $\mathrm{C} 11$ & $\mathrm{C} 16$ & $122.8(8)$ & C19' & $\mathrm{C} 21^{\prime}$ & $\mathrm{H} 21 \mathrm{~F}$ & 109.5 \\
\hline $\mathrm{C} 12$ & $\mathrm{C} 11$ & $\mathrm{C} 10$ & $117.5(9)$ & $\mathrm{H} 21 \mathrm{D}$ & $\mathrm{C} 21^{\prime}$ & $\mathrm{H} 21 \mathrm{E}$ & 109.5 \\
\hline $\mathrm{C} 12$ & $\mathrm{C} 11$ & $\mathrm{C} 16$ & 119.7(9) & $\mathrm{H} 21 \mathrm{D}$ & $\mathrm{C} 21^{\prime}$ & $\mathrm{H} 21 \mathrm{~F}$ & 109.5 \\
\hline $\mathrm{C} 11$ & $\mathrm{C} 16$ & H16 & 107.8 & $\mathrm{H} 21 \mathrm{E}$ & $\mathrm{C} 21^{\prime}$ & $\mathrm{H} 21 \mathrm{~F}$ & 109.5 \\
\hline $\mathrm{C} 11$ & $\mathrm{C} 16$ & $\mathrm{C} 17$ & $112.3(8)$ & C19' & $\mathrm{C} 20^{\prime}$ & $\mathrm{H} 20 \mathrm{D}$ & 109.5 \\
\hline $\mathrm{C} 17$ & $\mathrm{C} 16$ & H16 & 107.8 & C19' & $\mathrm{C} 20^{\prime}$ & $\mathrm{H} 20 \mathrm{E}$ & 109.5 \\
\hline $\mathrm{C} 18$ & $\mathrm{C} 16$ & C11 & $111.4(8)$ & C19' & $\mathrm{C} 20^{\prime}$ & $\mathrm{H} 20 \mathrm{~F}$ & 109.5 \\
\hline $\mathrm{C} 18$ & $\mathrm{C} 16$ & H16 & 107.8 & $\mathrm{H} 20 \mathrm{D}$ & $\mathrm{C} 20^{\prime}$ & $\mathrm{H} 20 \mathrm{E}$ & 109.5 \\
\hline $\mathrm{C} 18$ & $\mathrm{C} 16$ & $\mathrm{C} 17$ & $109.5(7)$ & $\mathrm{H} 20 \mathrm{D}$ & $\mathrm{C} 20^{\prime}$ & $\mathrm{H} 20 \mathrm{~F}$ & 109.5 \\
\hline $\mathrm{C} 16$ & $\mathrm{C} 17$ & $\mathrm{H} 17 \mathrm{~A}$ & 109.5 & $\mathrm{H} 20 \mathrm{E}$ & $\mathrm{C} 20^{\prime}$ & $\mathrm{H} 20 \mathrm{~F}$ & 109.5 \\
\hline $\mathrm{C} 16$ & $\mathrm{C} 17$ & H17B & 109.5 & $\mathrm{C} 11^{\prime}$ & $\mathrm{C} 16^{\prime}$ & H16' & 108.1 \\
\hline $\mathrm{C} 16$ & $\mathrm{C} 17$ & $\mathrm{H} 17 \mathrm{C}$ & 109.5 & $\mathrm{C} 11^{\prime}$ & $\mathrm{C} 16^{\prime}$ & $\mathrm{C} 17^{\prime}$ & $109.2(7)$ \\
\hline $\mathrm{H} 17 \mathrm{~A}$ & $\mathrm{C} 17$ & H17B & 109.5 & $\mathrm{C} 11^{\prime}$ & $\mathrm{C} 16^{\prime}$ & $\mathrm{C} 18^{\prime}$ & $114.3(8)$ \\
\hline H17A & $\mathrm{C} 17$ & $\mathrm{H} 17 \mathrm{C}$ & 109.5 & $\mathrm{C} 17^{\prime}$ & $\mathrm{C} 16^{\prime}$ & H16' & 108.1 \\
\hline H17B & $\mathrm{C} 17$ & $\mathrm{H} 17 \mathrm{C}$ & 109.5 & $\mathrm{C} 17^{\prime}$ & $\mathrm{C} 16^{\prime}$ & $\mathrm{C} 18^{\prime}$ & $108.9(8)$ \\
\hline $\mathrm{C} 16$ & $\mathrm{C} 18$ & $\mathrm{H} 18 \mathrm{~A}$ & 109.5 & $\mathrm{C} 18^{\prime}$ & $\mathrm{C} 16^{\prime}$ & H16' & 108.1 \\
\hline $\mathrm{C} 16$ & $\mathrm{C} 18$ & H18B & 109.5 & $\mathrm{C} 16^{\prime}$ & $\mathrm{C} 17^{\prime}$ & H17D & 109.5 \\
\hline $\mathrm{C} 16$ & $\mathrm{C} 18$ & $\mathrm{H} 18 \mathrm{C}$ & 109.5 & $\mathrm{C} 16^{\prime}$ & $\mathrm{C} 17^{\prime}$ & H17E & 109.5 \\
\hline $\mathrm{H} 18 \mathrm{~A}$ & $\mathrm{C} 18$ & H18B & 109.5 & $\mathrm{C} 16^{\prime}$ & $\mathrm{C} 17^{\prime}$ & $\mathrm{H} 17 \mathrm{~F}$ & 109.5 \\
\hline $\mathrm{H} 18 \mathrm{~A}$ & $\mathrm{C} 18$ & $\mathrm{H} 18 \mathrm{C}$ & 109.5 & H17D & $\mathrm{C} 17^{\prime}$ & H17E & 109.5 \\
\hline H18B & $\mathrm{C} 18$ & $\mathrm{H} 18 \mathrm{C}$ & 109.5 & H17D & $\mathrm{C} 17^{\prime}$ & $\mathrm{H} 17 \mathrm{~F}$ & 109.5 \\
\hline $\mathrm{C} 15$ & $\mathrm{C} 19$ & H19 & 108 & H17E & $\mathrm{C} 17^{\prime}$ & $\mathrm{H} 17 \mathrm{~F}$ & 109.5 \\
\hline $\mathrm{C} 21$ & $\mathrm{C} 19$ & $\mathrm{C} 15$ & 113.1(8) & $\mathrm{C} 16^{\prime}$ & $\mathrm{C} 18^{\prime}$ & $\mathrm{H} 18 \mathrm{D}$ & 109.5 \\
\hline $\mathrm{C} 21$ & $\mathrm{C} 19$ & H19 & 108 & C16' & $\mathrm{C} 18^{\prime}$ & $\mathrm{H} 18 \mathrm{E}$ & 109.5 \\
\hline $\mathrm{C} 21$ & $\mathrm{C} 19$ & $\mathrm{C} 20$ & $109.5(8)$ & C16' & $\mathrm{C} 18^{\prime}$ & $\mathrm{H} 18 \mathrm{~F}$ & 109.5 \\
\hline $\mathrm{C} 20$ & $\mathrm{C} 19$ & $\mathrm{C} 15$ & $110.0(8)$ & $\mathrm{H} 18 \mathrm{D}$ & $\mathrm{C} 18^{\prime}$ & $\mathrm{H} 18 \mathrm{E}$ & 109.5 \\
\hline $\mathrm{C} 20$ & $\mathrm{C} 19$ & H19 & 108 & $\mathrm{H} 18 \mathrm{D}$ & $\mathrm{C} 18^{\prime}$ & $\mathrm{H} 18 \mathrm{~F}$ & 109.5 \\
\hline $\mathrm{C} 19$ & $\mathrm{C} 21$ & $\mathrm{H} 21 \mathrm{~A}$ & 109.5 & $\mathrm{H} 18 \mathrm{E}$ & $\mathrm{C} 18^{\prime}$ & $\mathrm{H} 18 \mathrm{~F}$ & 109.5 \\
\hline $\mathrm{C} 19$ & $\mathrm{C} 21$ & $\mathrm{H} 21 \mathrm{~B}$ & 109.5 & C6' & C7' & H7'A & 109.5 \\
\hline $\mathrm{C} 19$ & $\mathrm{C} 21$ & $\mathrm{H} 21 \mathrm{C}$ & 109.5 & C6' & $\mathrm{C} 7^{\prime}$ & $\mathrm{H} 7 \mathrm{7}^{\prime} \mathrm{B}$ & 109.5 \\
\hline $\mathrm{H} 21 \mathrm{~A}$ & $\mathrm{C} 21$ & $\mathrm{H} 21 \mathrm{~B}$ & 109.5 & C6' & $\mathrm{C} 7^{\prime}$ & $\mathrm{H} 7^{\prime} \mathrm{C}$ & 109.5 \\
\hline $\mathrm{H} 21 \mathrm{~A}$ & $\mathrm{C} 21$ & $\mathrm{H} 21 \mathrm{C}$ & 109.5 & H7'A & $\mathrm{C} 7^{\prime}$ & H7'B & 109.5 \\
\hline $\mathrm{H} 21 \mathrm{~B}$ & $\mathrm{C} 21$ & $\mathrm{H} 21 \mathrm{C}$ & 109.5 & H7'A & C7' & $\mathrm{H} 7^{\prime} \mathrm{C}$ & 109.5 \\
\hline $\mathrm{C} 19$ & $\mathrm{C} 20$ & $\mathrm{H} 20 \mathrm{~A}$ & 109.5 & H7'B & $C 7^{\prime}$ & $\mathrm{H} 7^{\prime} \mathrm{C}$ & 109.5 \\
\hline $\mathrm{C} 19$ & $\mathrm{C} 20$ & H20B & 109.5 & C6' & C9' & H9'A & 109.5 \\
\hline $\mathrm{C} 19$ & $\mathrm{C} 20$ & $\mathrm{H} 20 \mathrm{C}$ & 109.5 & C6' & C9' & H9'B & 109.5 \\
\hline $\mathrm{H} 20 \mathrm{~A}$ & $\mathrm{C} 20$ & H20B & 109.5 & C6' & C9' & $\mathrm{H}^{\prime} \mathrm{C}$ & 109.5 \\
\hline $\mathrm{H} 20 \mathrm{~A}$ & $\mathrm{C} 20$ & $\mathrm{H} 20 \mathrm{C}$ & 109.5 & H9'A & C9' & H9'B & 109.5 \\
\hline $\mathrm{H} 20 \mathrm{~B}$ & $\mathrm{C} 20$ & $\mathrm{H} 20 \mathrm{C}$ & 109.5 & H9'A & C9' & $\mathrm{H} 9^{\prime} \mathrm{C}$ & 109.5 \\
\hline
\end{tabular}




$\begin{array}{lccccccc}\text { C7 } & \text { C6 } & \text { C5 } & 109.5(7) & \text { H9'B }^{\prime} & \text { C9' }^{\prime} & \text { H9'C } & 109.5 \\ \text { C7 } & \text { C6 } & \text { C8 } & 111.0(7) & \text { C2' }^{\prime} & \text { C22' } & \text { H22D } & 109.5 \\ \text { C9 } & \text { C6 } & \text { C5 } & 113.8(7) & \text { C2' }^{\prime} & \text { C22' } & \text { H22E } & 109.5 \\ \text { C9 } & \text { C6 } & \text { C7 } & 107.1(7) & \text { C2' }^{\prime} & \text { C22' } & \text { H22F } & 109.5 \\ \text { C9 } & \text { C6 } & \text { C8 } & 106.0(7) & \text { H22D } & \text { C22' } & \text { H22E } & 109.5 \\ \text { C8 } & \text { C6 } & \text { C5 } & 109.4(7) & \text { H22D } & \text { C22' } & \text { H22F } & 109.5 \\ \text { C6 } & \text { C7 } & \text { H7A } & 109.5 & \text { H22E } & \text { C22' } & \text { H22F } & 109.5\end{array}$

Table S6.2.4. Torsion angles for (6e)AuCl.

\begin{tabular}{|c|c|c|c|c|}
\hline Atom & Atom & Atom & Atom & Torsion Angle $/{ }^{\circ}$ \\
\hline $\mathrm{Au} 1$ & $\mathrm{C} 1$ & $\mathrm{C} 2$ & $\mathrm{C} 3$ & $179.3(6)$ \\
\hline $\mathrm{Au} 1$ & $\mathrm{C} 1$ & $\mathrm{C} 2$ & $\mathrm{C} 22$ & $-62.6(9)$ \\
\hline $\mathrm{Au} 1$ & $\mathrm{C} 1$ & $\mathrm{C} 2$ & $\mathrm{C} 5$ & $64.5(9)$ \\
\hline Au1' & $\mathrm{C} 1^{\prime}$ & $\mathrm{C} 2^{\prime}$ & C $3^{\prime}$ & $179.5(7)$ \\
\hline Au1' & $\mathrm{C} 1^{\prime}$ & $\mathrm{C} 2^{\prime}$ & $\mathrm{C}^{\prime}$ & $62.1(9)$ \\
\hline Au1' & $\mathrm{C} 1^{\prime}$ & $\mathrm{C} 2{ }^{\prime}$ & $\mathrm{C} 22^{\prime}$ & $-65.7(8)$ \\
\hline N1 & $\mathrm{C} 1$ & $\mathrm{C} 2$ & $\mathrm{C} 3$ & $-8.8(10)$ \\
\hline N1 & $\mathrm{C} 1$ & $\mathrm{C} 2$ & $\mathrm{C} 22$ & $109.3(8)$ \\
\hline $\mathrm{N} 1$ & $\mathrm{C} 1$ & $\mathrm{C} 2$ & $\mathrm{C} 5$ & $-123.6(8)$ \\
\hline $\mathrm{N} 1$ & $\mathrm{C} 25$ & $\mathrm{C} 26$ & $\mathrm{C} 31$ & $8.7(12)$ \\
\hline $\mathrm{N} 1$ & $\mathrm{C} 25$ & $\mathrm{C} 26$ & $\mathrm{C} 27$ & $-177.5(7)$ \\
\hline N1 & $\mathrm{C} 25$ & $\mathrm{C} 30$ & $\mathrm{C} 29$ & $176.7(7)$ \\
\hline $\mathrm{N} 1$ & $\mathrm{C} 25$ & $\mathrm{C} 30$ & $\mathrm{C} 34$ & $-9.8(12)$ \\
\hline N1 & $\mathrm{C} 4$ & $\mathrm{C} 3$ & $\mathrm{C} 2$ & $-13.0(10)$ \\
\hline $\mathrm{N} 2$ & $\mathrm{C} 5$ & C6 & $\mathrm{C} 7$ & $-113.3(10)$ \\
\hline $\mathrm{N} 2$ & $\mathrm{C} 5$ & C6 & C9 & $6.5(13)$ \\
\hline $\mathrm{N} 2$ & $\mathrm{C} 5$ & C6 & $\mathrm{C} 8$ & $124.8(10)$ \\
\hline $\mathrm{N} 2$ & $\mathrm{C} 10$ & $\mathrm{C} 15$ & $\mathrm{C} 14$ & $-174.1(9)$ \\
\hline $\mathrm{N} 2$ & $\mathrm{C} 10$ & $\mathrm{C} 15$ & $\mathrm{C} 19$ & $5.7(15)$ \\
\hline $\mathrm{N} 2$ & $\mathrm{C} 10$ & $\mathrm{C} 11$ & $\mathrm{C} 12$ & $174.2(9)$ \\
\hline $\mathrm{N} 2$ & $\mathrm{C} 10$ & $\mathrm{C} 11$ & $\mathrm{C} 16$ & $-4.6(14)$ \\
\hline N1' & $\mathrm{C} 25^{\prime}$ & $\mathrm{C} 30^{\prime}$ & C34' & $-10.8(12)$ \\
\hline N1' & $\mathrm{C} 25^{\prime}$ & $\mathrm{C} 30^{\prime}$ & $\mathrm{C} 29^{\prime}$ & $176.0(7)$ \\
\hline N1' & $\mathrm{C} 25^{\prime}$ & $\mathrm{C} 26^{\prime}$ & $\mathrm{C} 27^{\prime}$ & $-176.4(7)$ \\
\hline N1' & $\mathrm{C} 25^{\prime}$ & $\mathrm{C} 26^{\prime}$ & $\mathrm{C} 31^{\prime}$ & $6.5(11)$ \\
\hline N1' & $\mathrm{C} 1^{\prime}$ & $\mathrm{C} 2^{\prime}$ & $\mathrm{C} 3^{\prime}$ & $-9.6(9)$ \\
\hline N1' & $\mathrm{C} 1^{\prime}$ & $\mathrm{C} 2^{\prime}$ & $\mathrm{C} 5^{\prime}$ & $-126.9(8)$ \\
\hline $\mathrm{N} 1^{\prime}$ & $\mathrm{C} 1^{\prime}$ & $\mathrm{C} 2{ }^{\prime}$ & $\mathrm{C} 22^{\prime}$ & $105.3(8)$ \\
\hline N1' & $\mathrm{C}^{\prime}$ & $\mathrm{C} 3^{\prime}$ & $\mathrm{C} 2^{\prime}$ & $-16.1(9)$ \\
\hline $\mathrm{C} 25^{\prime}$ & N1' & $\mathrm{C} 1^{\prime}$ & Au1' & $-8.1(13)$ \\
\hline $\mathrm{C} 25^{\prime}$ & N1' & $\mathrm{C} 1^{\prime}$ & $\mathrm{C} 2^{\prime}$ & $-178.8(8)$ \\
\hline $\mathrm{C} 25^{\prime}$ & $\mathrm{N} 1^{\prime}$ & $\mathrm{C} 4^{\prime}$ & $\mathrm{C} 24^{\prime}$ & $69.7(10)$ \\
\hline $\mathrm{C} 25^{\prime}$ & N1' & $\mathrm{C}^{\prime}$ & $\mathrm{C} 23^{\prime}$ & $-50.6(10)$ \\
\hline
\end{tabular}




\begin{tabular}{|c|c|c|c|c|}
\hline $\mathrm{C} 25^{\prime}$ & N1' & $\mathrm{C}^{\prime}$ & $\mathrm{C} 3^{\prime}$ & $-171.2(7)$ \\
\hline $\mathrm{C} 25^{\prime}$ & $\mathrm{C} 30^{\prime}$ & C34' & $\mathrm{C} 36^{\prime}$ & $126.2(8)$ \\
\hline $\mathrm{C} 25^{\prime}$ & $\mathrm{C} 30^{\prime}$ & $\mathrm{C} 34^{\prime}$ & $\mathrm{C} 35^{\prime}$ & $-111.3(9)$ \\
\hline $\mathrm{C} 25^{\prime}$ & $\mathrm{C} 30^{\prime}$ & $\mathrm{C} 29^{\prime}$ & $\mathrm{C} 28^{\prime}$ & $2.6(12)$ \\
\hline $\mathrm{C} 25^{\prime}$ & $\mathrm{C} 26^{\prime}$ & $\mathrm{C} 31^{\prime}$ & C33' & $-135.4(8)$ \\
\hline $\mathrm{C} 25^{\prime}$ & $\mathrm{C} 26^{\prime}$ & $\mathrm{C} 31^{\prime}$ & $\mathrm{C} 32^{\prime}$ & $98.6(9)$ \\
\hline $\mathrm{N} 2^{\prime}$ & $\mathrm{C} 10^{\prime}$ & $\mathrm{C} 15^{\prime}$ & $\mathrm{C} 19^{\prime}$ & $-4.3(14)$ \\
\hline $\mathrm{N} 2^{\prime}$ & $\mathrm{C} 10^{\prime}$ & $\mathrm{C} 15^{\prime}$ & $\mathrm{C} 14^{\prime}$ & $176.2(9)$ \\
\hline $\mathrm{C} 11^{\prime}$ & $\mathrm{C} 10^{\prime}$ & $\mathrm{C} 15^{\prime}$ & $\mathrm{C} 19^{\prime}$ & $-177.8(8)$ \\
\hline C11' & $\mathrm{C} 10^{\prime}$ & $\mathrm{C} 15^{\prime}$ & $\mathrm{C} 14^{\prime}$ & $2.7(14)$ \\
\hline C11' & $\mathrm{C} 12^{\prime}$ & $\mathrm{C} 13^{\prime}$ & $\mathrm{C} 14^{\prime}$ & $2.4(14)$ \\
\hline $\mathrm{C} 1$ & $\mathrm{~N} 1$ & $\mathrm{C} 25$ & $\mathrm{C} 26$ & $-90.4(11)$ \\
\hline $\mathrm{C} 1$ & N1 & $\mathrm{C} 25$ & $\mathrm{C} 30$ & $89.0(11)$ \\
\hline $\mathrm{C} 1$ & $\mathrm{~N} 1$ & $\mathrm{C} 4$ & $\mathrm{C} 23$ & $127.6(9)$ \\
\hline $\mathrm{C} 1$ & N1 & $\mathrm{C} 4$ & $\mathrm{C} 24$ & $-110.9(9)$ \\
\hline $\mathrm{C} 1$ & N1 & $\mathrm{C} 4$ & $\mathrm{C} 3$ & $8.4(11)$ \\
\hline $\mathrm{C} 1$ & $\mathrm{C} 2$ & $\mathrm{C} 5$ & N2 & $29.4(10)$ \\
\hline $\mathrm{C} 1$ & $\mathrm{C} 2$ & $\mathrm{C} 5$ & C6 & $-154.0(7)$ \\
\hline $\mathrm{C} 25$ & $\mathrm{~N} 1$ & $\mathrm{C} 1$ & $\mathrm{Au} 1$ & $-6.5(14)$ \\
\hline $\mathrm{C} 25$ & N1 & $\mathrm{C} 1$ & $\mathrm{C} 2$ & $-177.8(8)$ \\
\hline $\mathrm{C} 25$ & N1 & $\mathrm{C} 4$ & $\mathrm{C} 23$ & $-54.3(11)$ \\
\hline $\mathrm{C} 25$ & N1 & $\mathrm{C} 4$ & $\mathrm{C} 24$ & $67.2(11)$ \\
\hline $\mathrm{C} 25$ & N1 & $\mathrm{C} 4$ & $\mathrm{C} 3$ & $-173.5(8)$ \\
\hline $\mathrm{C} 25$ & $\mathrm{C} 26$ & $\mathrm{C} 31$ & $\mathrm{C} 33$ & $-134.2(9)$ \\
\hline $\mathrm{C} 25$ & $\mathrm{C} 26$ & $\mathrm{C} 31$ & $\mathrm{C} 32$ & $103.5(10)$ \\
\hline $\mathrm{C} 25$ & $\mathrm{C} 26$ & $\mathrm{C} 27$ & $\mathrm{C} 28$ & $0.3(12)$ \\
\hline $\mathrm{C} 25$ & $\mathrm{C} 30$ & $\mathrm{C} 34$ & $\mathrm{C} 35$ & $-110.8(9)$ \\
\hline $\mathrm{C} 25$ & $\mathrm{C} 30$ & $\mathrm{C} 34$ & $\mathrm{C} 36$ & $127.5(9)$ \\
\hline $\mathrm{C} 26$ & $\mathrm{C} 25$ & $\mathrm{C} 30$ & $\mathrm{C} 29$ & $-4.0(12)$ \\
\hline $\mathrm{C} 26$ & $\mathrm{C} 25$ & $\mathrm{C} 30$ & $\mathrm{C} 34$ & $169.6(8)$ \\
\hline $\mathrm{C} 26$ & $\mathrm{C} 27$ & $\mathrm{C} 28$ & $\mathrm{C} 29$ & $-2.6(13)$ \\
\hline C31 & $\mathrm{C} 26$ & $\mathrm{C} 27$ & $\mathrm{C} 28$ & 174.3(8) \\
\hline $\mathrm{C} 27$ & $\mathrm{C} 26$ & $\mathrm{C} 31$ & $\mathrm{C} 33$ & $52.2(11)$ \\
\hline $\mathrm{C} 27$ & $\mathrm{C} 26$ & $\mathrm{C} 31$ & $\mathrm{C} 32$ & $-70.1(10)$ \\
\hline $\mathrm{C} 27$ & $\mathrm{C} 28$ & $\mathrm{C} 29$ & $\mathrm{C} 30$ & $1.7(13)$ \\
\hline $\mathrm{C} 28$ & $\mathrm{C} 29$ & $\mathrm{C} 30$ & $\mathrm{C} 25$ & $1.5(12)$ \\
\hline $\mathrm{C} 28$ & $\mathrm{C} 29$ & $\mathrm{C} 30$ & $\mathrm{C} 34$ & $-172.5(8)$ \\
\hline $\mathrm{C} 29$ & $\mathrm{C} 30$ & $\mathrm{C} 34$ & $\mathrm{C} 35$ & $62.7(9)$ \\
\hline $\mathrm{C} 29$ & $\mathrm{C} 30$ & $\mathrm{C} 34$ & $\mathrm{C} 36$ & $-59.0(10)$ \\
\hline $\mathrm{C} 30$ & $\mathrm{C} 25$ & $\mathrm{C} 26$ & $\mathrm{C} 31$ & $-170.7(8)$ \\
\hline C30 & $\mathrm{C} 25$ & $\mathrm{C} 26$ & $\mathrm{C} 27$ & $3.1(12)$ \\
\hline $\mathrm{C} 4$ & N1 & $\mathrm{C} 1$ & Au1 & $171.6(7)$ \\
\hline $\mathrm{C} 4$ & N1 & $\mathrm{C} 1$ & $\mathrm{C} 2$ & $0.3(11)$ \\
\hline $\mathrm{C} 4$ & $\mathrm{~N} 1$ & $\mathrm{C} 25$ & $\mathrm{C} 26$ & $91.7(10)$ \\
\hline
\end{tabular}




\begin{tabular}{|c|c|c|c|c|}
\hline $\mathrm{C} 4$ & $\mathrm{~N} 1$ & $\mathrm{C} 25$ & $\mathrm{C} 30$ & $-88.9(10)$ \\
\hline $\mathrm{C} 4$ & $\mathrm{C} 3$ & $\mathrm{C} 2$ & $\mathrm{C} 1$ & $13.6(10)$ \\
\hline $\mathrm{C} 4$ & $\mathrm{C} 3$ & $\mathrm{C} 2$ & $\mathrm{C} 22$ & $-99.3(9)$ \\
\hline $\mathrm{C} 4$ & $\mathrm{C} 3$ & $\mathrm{C} 2$ & $\mathrm{C} 5$ & $126.8(8)$ \\
\hline $\mathrm{C} 23$ & $\mathrm{C} 4$ & $\mathrm{C} 3$ & $\mathrm{C} 2$ & $-132.3(8)$ \\
\hline $\mathrm{C} 24$ & $\mathrm{C} 4$ & $\mathrm{C} 3$ & $\mathrm{C} 2$ & $103.5(9)$ \\
\hline $\mathrm{C} 3$ & $\mathrm{C} 2$ & $\mathrm{C} 5$ & $\mathrm{~N} 2$ & $-82.2(9)$ \\
\hline $\mathrm{C} 3$ & $\mathrm{C} 2$ & $\mathrm{C} 5$ & $\mathrm{C} 6$ & $94.5(9)$ \\
\hline $\mathrm{C} 2$ & $\mathrm{C} 5$ & $\mathrm{C} 6$ & $\mathrm{C} 7$ & $70.7(10)$ \\
\hline $\mathrm{C} 2$ & $\mathrm{C} 5$ & $\mathrm{C} 6$ & $\mathrm{C} 9$ & $-169.5(7)$ \\
\hline $\mathrm{C} 2$ & $\mathrm{C} 5$ & $\mathrm{C} 6$ & $\mathrm{C} 8$ & $-51.2(10)$ \\
\hline $\mathrm{C} 22$ & $\mathrm{C} 2$ & $\mathrm{C} 5$ & N2 & $147.6(8)$ \\
\hline $\mathrm{C} 22$ & $\mathrm{C} 2$ & $\mathrm{C} 5$ & $\mathrm{C} 6$ & $-35.8(11)$ \\
\hline $\mathrm{C} 5$ & $\mathrm{~N} 2$ & $\mathrm{C} 10$ & $\mathrm{C} 15$ & $-89.8(12)$ \\
\hline $\mathrm{C} 5$ & $\mathrm{~N} 2$ & $\mathrm{C} 10$ & $\mathrm{C} 11$ & $97.9(13)$ \\
\hline $\mathrm{C} 10$ & $\mathrm{~N} 2$ & $\mathrm{C} 5$ & $\mathrm{C} 2$ & $174.4(9)$ \\
\hline $\mathrm{C} 10$ & $\mathrm{~N} 2$ & $\mathrm{C} 5$ & $\mathrm{C} 6$ & $-1.8(16)$ \\
\hline $\mathrm{C} 10$ & $\mathrm{C} 15$ & $\mathrm{C} 14$ & $\mathrm{C} 13$ & $-0.1(15)$ \\
\hline $\mathrm{C} 10$ & $\mathrm{C} 15$ & $\mathrm{C} 19$ & $\mathrm{C} 21$ & $130.1(10)$ \\
\hline $\mathrm{C} 10$ & $\mathrm{C} 15$ & $\mathrm{C} 19$ & $\mathrm{C} 20$ & $-107.1(11)$ \\
\hline $\mathrm{C} 10$ & $\mathrm{C} 11$ & $\mathrm{C} 16$ & $\mathrm{C} 17$ & $123.2(10)$ \\
\hline $\mathrm{C} 10$ & $\mathrm{C} 11$ & $\mathrm{C} 16$ & $\mathrm{C} 18$ & $-113.6(10)$ \\
\hline $\mathrm{C} 15$ & $\mathrm{C} 10$ & $\mathrm{C} 11$ & $\mathrm{C} 12$ & $1.7(15)$ \\
\hline $\mathrm{C} 15$ & $\mathrm{C} 10$ & $\mathrm{C} 11$ & $\mathrm{C} 16$ & $-177.0(9)$ \\
\hline $\mathrm{C} 15$ & $\mathrm{C} 14$ & $\mathrm{C} 13$ & $\mathrm{C} 12$ & $1.9(16)$ \\
\hline $\mathrm{C} 14$ & $\mathrm{C} 15$ & $\mathrm{C} 19$ & $\mathrm{C} 21$ & $-50.1(12)$ \\
\hline $\mathrm{C} 14$ & $\mathrm{C} 15$ & $\mathrm{C} 19$ & $\mathrm{C} 20$ & $72.7(11)$ \\
\hline $\mathrm{C} 14$ & $\mathrm{C} 13$ & $\mathrm{C} 12$ & $\mathrm{C} 11$ & $-1.9(17)$ \\
\hline $\mathrm{C} 13$ & $\mathrm{C} 12$ & $\mathrm{C} 11$ & $\mathrm{C} 10$ & $0.1(16)$ \\
\hline $\mathrm{C} 13$ & $\mathrm{C} 12$ & $\mathrm{C} 11$ & $\mathrm{C} 16$ & $178.9(10)$ \\
\hline $\mathrm{C} 12$ & $\mathrm{C} 11$ & $\mathrm{C} 16$ & $\mathrm{C} 17$ & $-55.5(12)$ \\
\hline $\mathrm{C} 12$ & $\mathrm{C} 11$ & $\mathrm{C} 16$ & $\mathrm{C} 18$ & $67.7(12)$ \\
\hline $\mathrm{C} 11$ & $\mathrm{C} 10$ & $\mathrm{C} 15$ & $\mathrm{C} 14$ & $-1.8(15)$ \\
\hline C11 & $\mathrm{C} 10$ & $\mathrm{C} 15$ & $\mathrm{C} 19$ & $178.0(9)$ \\
\hline C19 & $\mathrm{C} 15$ & $\mathrm{C} 14$ & $\mathrm{C} 13$ & $-179.9(9)$ \\
\hline $\mathrm{C} 1^{\prime}$ & N1' & $\mathrm{C} 25^{\prime}$ & $\mathrm{C} 30^{\prime}$ & $88.4(11)$ \\
\hline $\mathrm{C} 1^{\prime}$ & N1' & $\mathrm{C} 25^{\prime}$ & $\mathrm{C} 26^{\prime}$ & $-90.1(10)$ \\
\hline $\mathrm{C} 1^{\prime}$ & N1' & $\mathrm{C} 4{ }^{\prime}$ & $\mathrm{C} 24^{\prime}$ & $-108.3(9)$ \\
\hline $\mathrm{C} 1^{\prime}$ & $\mathrm{N} 1^{\prime}$ & C4' & $\mathrm{C} 23^{\prime}$ & $131.3(8)$ \\
\hline $\mathrm{C} 1^{\prime}$ & N1' & C4' & C3' & $10.8(10)$ \\
\hline $\mathrm{C} 1^{\prime}$ & $\mathrm{C} 2^{\prime}$ & $\mathrm{C} 5^{\prime}$ & N2' & $29.8(10)$ \\
\hline $\mathrm{C} 1^{\prime}$ & $\mathrm{C} 2^{\prime}$ & $\mathrm{C} 5^{\prime}$ & C6' & $-153.8(7)$ \\
\hline C $30^{\prime}$ & $\mathrm{C} 25^{\prime}$ & $\mathrm{C} 26^{\prime}$ & $\mathrm{C} 27^{\prime}$ & $5.1(12)$ \\
\hline $\mathrm{C} 30^{\prime}$ & $\mathrm{C} 25^{\prime}$ & $\mathrm{C} 26^{\prime}$ & $\mathrm{C} 31^{\prime}$ & $-172.1(7)$ \\
\hline
\end{tabular}




\begin{tabular}{|c|c|c|c|c|}
\hline $\mathrm{C} 30^{\prime}$ & $\mathrm{C} 29^{\prime}$ & $\mathrm{C} 28^{\prime}$ & $\mathrm{C} 27^{\prime}$ & $0.7(13)$ \\
\hline C34' & $\mathrm{C} 30^{\prime}$ & $\mathrm{C} 29^{\prime}$ & $\mathrm{C} 28^{\prime}$ & $-171.1(8)$ \\
\hline C29' & $\mathrm{C} 30^{\prime}$ & $\mathrm{C} 34^{\prime}$ & $\mathrm{C} 36^{\prime}$ & $-60.7(10)$ \\
\hline C29' & $\mathrm{C} 30^{\prime}$ & $\mathrm{C} 34^{\prime}$ & $\mathrm{C} 35^{\prime}$ & $61.9(9)$ \\
\hline C29' & $\mathrm{C} 28^{\prime}$ & $\mathrm{C} 27^{\prime}$ & $\mathrm{C} 26^{\prime}$ & $-1.2(13)$ \\
\hline $\mathrm{C} 28^{\prime}$ & $\mathrm{C} 27^{\prime}$ & $\mathrm{C} 26^{\prime}$ & $\mathrm{C} 25^{\prime}$ & $-1.6(12)$ \\
\hline $\mathrm{C} 28^{\prime}$ & $\mathrm{C} 27^{\prime}$ & $\mathrm{C} 26^{\prime}$ & $\mathrm{C} 31^{\prime}$ & $175.6(7)$ \\
\hline $\mathrm{C} 27^{\prime}$ & $\mathrm{C} 26^{\prime}$ & $\mathrm{C} 31^{\prime}$ & C33' & $47.5(11)$ \\
\hline $\mathrm{C} 27^{\prime}$ & $\mathrm{C} 26^{\prime}$ & $\mathrm{C} 31^{\prime}$ & C $32^{\prime}$ & $-78.5(9)$ \\
\hline $\mathrm{C} 26^{\prime}$ & $\mathrm{C} 25^{\prime}$ & $\mathrm{C} 30^{\prime}$ & C34' & $167.8(7)$ \\
\hline $\mathrm{C} 26^{\prime}$ & $\mathrm{C} 25^{\prime}$ & $\mathrm{C} 30^{\prime}$ & $\mathrm{C} 29^{\prime}$ & $-5.5(12)$ \\
\hline $\mathrm{C} 4^{\prime}$ & $\mathrm{N} 11^{\prime}$ & $\mathrm{C} 25^{\prime}$ & $\mathrm{C} 30^{\prime}$ & $-89.5(9)$ \\
\hline $\mathrm{C} 4^{\prime}$ & $\mathrm{N} 11^{\prime}$ & $\mathrm{C} 25^{\prime}$ & $\mathrm{C} 26^{\prime}$ & $92.0(9)$ \\
\hline $\mathrm{C} 4^{\prime}$ & N1' & $\mathrm{C} 1{ }^{\prime}$ & Aul' & $169.9(6)$ \\
\hline $\mathrm{C} 4^{\prime}$ & N1' & $\mathrm{C} 1^{\prime}$ & $\mathrm{C} 2^{\prime}$ & $-0.8(10)$ \\
\hline $\mathrm{C} 4^{\prime}$ & $\mathrm{C} 3^{\prime}$ & $\mathrm{C} 2^{\prime}$ & $\mathrm{C} 11^{\prime}$ & $16.4(9)$ \\
\hline $\mathrm{C} 4^{\prime}$ & $\mathrm{C} 3^{\prime}$ & $\mathrm{C} 2{ }^{\prime}$ & $\mathrm{C} 5^{\prime}$ & $133.0(8)$ \\
\hline $\mathrm{C} 4^{\prime}$ & C3' & $\mathrm{C} 2^{\prime}$ & $\mathrm{C} 22^{\prime}$ & $-92.8(9)$ \\
\hline $\mathrm{C} 24^{\prime}$ & $\mathrm{C} 4^{\prime}$ & C3' & $\mathrm{C} 2^{\prime}$ & $99.6(9)$ \\
\hline $\mathrm{C} 23^{\prime}$ & $\mathrm{C} 4^{\prime}$ & C3' & $\mathrm{C} 2^{\prime}$ & $-136.0(8)$ \\
\hline C3' & $\mathrm{C} 2^{\prime}$ & $\mathrm{C} 5^{\prime}$ & $\mathrm{N} 2{ }^{\prime}$ & $-82.5(9)$ \\
\hline C3' & $\mathrm{C} 2{ }^{\prime}$ & $\mathrm{C} 5^{\prime}$ & C6' & $94.0(8)$ \\
\hline $\mathrm{C} 8^{\prime}$ & $\mathrm{C} 6^{\prime}$ & $\mathrm{C} 5^{\prime}$ & $\mathrm{N} 2{ }^{\prime}$ & $126.9(9)$ \\
\hline $\mathrm{C} 8^{\prime}$ & C6' & $\mathrm{C}^{\prime}$ & $\mathrm{C} 2^{\prime}$ & $-49.0(9)$ \\
\hline $\mathrm{C} 5^{\prime}$ & $\mathrm{N} 2^{\prime}$ & $\mathrm{C} 10^{\prime}$ & $\mathrm{C} 11^{\prime}$ & $-86.8(12)$ \\
\hline $\mathrm{C}^{\prime}$ & $\mathrm{N} 2{ }^{\prime}$ & $\mathrm{C} 10^{\prime}$ & $\mathrm{C} 15^{\prime}$ & $99.7(12)$ \\
\hline $\mathrm{C} 10^{\prime}$ & $\mathrm{N} 2^{\prime}$ & $\mathrm{C} 5^{\prime}$ & $\mathrm{C} 2^{\prime}$ & $175.1(8)$ \\
\hline $\mathrm{C} 10^{\prime}$ & $\mathrm{N} 2{ }^{\prime}$ & $\mathrm{C} 5^{\prime}$ & $\mathrm{C} 6^{\prime}$ & $-1.0(15)$ \\
\hline $\mathrm{C} 10^{\prime}$ & $\mathrm{C} 11^{\prime}$ & $\mathrm{C} 12^{\prime}$ & $\mathrm{C} 13^{\prime}$ & $-0.9(14)$ \\
\hline $\mathrm{C} 10^{\prime}$ & $\mathrm{C} 11^{\prime}$ & $\mathrm{C} 16^{\prime}$ & $\mathrm{C} 17^{\prime}$ & $-83.8(10)$ \\
\hline $\mathrm{C} 10^{\prime}$ & $\mathrm{C} 11^{\prime}$ & $\mathrm{C} 16^{\prime}$ & $\mathrm{C} 18^{\prime}$ & 153.9(9) \\
\hline $\mathrm{C} 12^{\prime}$ & $\mathrm{C} 11^{\prime}$ & $\mathrm{C} 10^{\prime}$ & $\mathrm{N} 2^{\prime}$ & $-175.1(8)$ \\
\hline $\mathrm{C} 12^{\prime}$ & $\mathrm{C} 11^{\prime}$ & $\mathrm{C} 10^{\prime}$ & $\mathrm{C} 15^{\prime}$ & $-1.6(14)$ \\
\hline $\mathrm{C} 12^{\prime}$ & $\mathrm{C} 11^{\prime}$ & $\mathrm{C} 16^{\prime}$ & $\mathrm{C} 17^{\prime}$ & $94.2(10)$ \\
\hline $\mathrm{C} 12^{\prime}$ & $\mathrm{C} 11^{\prime}$ & $\mathrm{C} 16^{\prime}$ & $\mathrm{C} 18^{\prime}$ & $-28.0(12)$ \\
\hline $\mathrm{C} 12^{\prime}$ & $\mathrm{C} 13^{\prime}$ & $\mathrm{C} 14^{\prime}$ & $\mathrm{C} 15^{\prime}$ & $-1.3(14)$ \\
\hline $\mathrm{C} 13^{\prime}$ & $\mathrm{C} 14^{\prime}$ & $\mathrm{C} 15^{\prime}$ & $\mathrm{C} 19^{\prime}$ & $179.2(8)$ \\
\hline $\mathrm{C} 13^{\prime}$ & $\mathrm{C} 14$ & $\mathrm{C} 15^{\prime}$ & $\mathrm{C} 10^{\prime}$ & $-1.2(14)$ \\
\hline $\mathrm{C} 21^{\prime}$ & $\mathrm{C} 19^{\prime}$ & $\mathrm{C} 15^{\prime}$ & $\mathrm{C} 10^{\prime}$ & $-112.3(10)$ \\
\hline $\mathrm{C} 21^{\prime}$ & $\mathrm{C} 19^{\prime}$ & $\mathrm{C} 15^{\prime}$ & $\mathrm{C} 14^{\prime}$ & $67.2(11)$ \\
\hline $\mathrm{C} 20^{\prime}$ & $\mathrm{C} 19^{\prime}$ & $\mathrm{C} 15^{\prime}$ & $\mathrm{C} 10^{\prime}$ & $123.8(9)$ \\
\hline $\mathrm{C} 20^{\prime}$ & $\mathrm{C} 19^{\prime}$ & $\mathrm{C} 15^{\prime}$ & C14' & $-56.7(11)$ \\
\hline $\mathrm{C} 16^{\prime}$ & $\mathrm{C} 11^{\prime}$ & $\mathrm{C} 10^{\prime}$ & $\mathrm{N} 2^{\prime}$ & $3.0(14)$ \\
\hline C16' & $\mathrm{C} 11^{\prime}$ & $\mathrm{C} 10^{\prime}$ & $\mathrm{C} 15^{\prime}$ & $176.5(8)$ \\
\hline
\end{tabular}




\begin{tabular}{|c|c|c|c|c|}
\hline $\mathrm{C} 16^{\prime}$ & $\mathrm{C} 11^{\prime}$ & $\mathrm{C} 12^{\prime}$ & C13' & $-179.0(8)$ \\
\hline C7' & C6' & $\mathrm{C} 5^{\prime}$ & $\mathrm{N} 2{ }^{\prime}$ & $-112.4(10)$ \\
\hline C7' & $\mathrm{C} 6^{\prime}$ & $\mathrm{C} 5^{\prime}$ & $\mathrm{C} 2{ }^{\prime}$ & 71.8(9) \\
\hline C9' & C6' & $\mathrm{C} 5^{\prime}$ & $\mathrm{N} 2^{\prime}$ & $7.5(12)$ \\
\hline C9' & C6' & $\mathrm{C} 5^{\prime}$ & $\mathrm{C} 2{ }^{\prime}$ & $-168.3(7)$ \\
\hline $\mathrm{C} 22^{\prime}$ & $\mathrm{C} 2{ }^{\prime}$ & $\mathrm{C} 5^{\prime}$ & $\mathrm{N} 2{ }^{\prime}$ & $147.8(8)$ \\
\hline $\mathrm{C} 22^{\prime}$ & $\mathrm{C} 22^{\prime}$ & $\mathrm{C} 5^{\prime}$ & C6' & $-35.8(11)$ \\
\hline
\end{tabular}

\subsection{Copper complex (6f)CuCl}

Single crystals of $\mathrm{C}_{20} \mathrm{H}_{30} \mathrm{ClCuIN}(\mathbf{6 f}) \mathbf{C u C l}$ were grown by slow diffusion of hexane into a solution in benzene. A suitable crystal was selected and mounted on a 'Bruker APEX-II CCD' diffractometer. The crystal was kept at $100 \mathrm{~K}$ during data collection. Using Olex $2{ }^{8}$ the structure was solved with the ShelXS ${ }^{9}$ structure solution program using Direct Methods and refined with the ShelXL ${ }^{9}$ refinement package using Least Squares minimization.

Table S 6.3.1. Crystal data and structure refinement for $(6 f) \mathrm{CuCl}$.

\begin{tabular}{|c|c|}
\hline Identification code & $(6 f) \mathrm{CuCl}$ \\
\hline Empirical formula & $\mathrm{C}_{20} \mathrm{H}_{30} \mathrm{ClCuIN}$ \\
\hline Formula weight & 510.34 \\
\hline Temperature/K & 100 \\
\hline Crystal system & monoclinic \\
\hline Space group & $\mathrm{P} 2_{1} / \mathrm{c}$ \\
\hline $\mathrm{a} / \AA$ & $10.0336(6)$ \\
\hline $\mathrm{b} / \AA$ & $9.9876(6)$ \\
\hline $\mathrm{c} / \AA$ & $21.8458(15)$ \\
\hline$\alpha /^{\circ}$ & 90 \\
\hline$\beta /{ }^{\circ}$ & $95.333(3)$ \\
\hline$\gamma /{ }^{\circ}$ & 90 \\
\hline Volume $/ \AA^{3}$ & $2179.7(2)$ \\
\hline Z & 4 \\
\hline$\rho_{\text {calc }} \mathrm{g} / \mathrm{cm}^{3}$ & 1.555 \\
\hline$\mu / \mathrm{mm}^{-1}$ & 2.543 \\
\hline $\mathrm{F}(000)$ & 1024.0 \\
\hline Crystal size $/ \mathrm{mm}^{3}$ & $0.216 \times 0.127 \times 0.089$ \\
\hline Radiation & $\operatorname{MoK} \alpha(\lambda=0.71073)$ \\
\hline $2 \Theta$ range for data collection ${ }^{\circ}$ & 3.744 to 50.708 \\
\hline Index ranges & $-9 \leq \mathrm{h} \leq 12,-11 \leq \mathrm{k} \leq 12,-26 \leq 1 \leq 19$ \\
\hline Reflections collected & 14484 \\
\hline Independent reflections & $3950\left[\mathrm{R}_{\mathrm{int}}=0.0373, \mathrm{R}_{\text {sigma }}=0.0391\right]$ \\
\hline Data/restraints/parameters & $3950 / 0 / 224$ \\
\hline Goodness-of-fit on $\mathrm{F}^{2}$ & 1.149 \\
\hline Final $R$ indexes $[I>=2 \sigma(I)]$ & $\mathrm{R}_{1}=0.0563, \mathrm{wR}_{2}=0.1452$ \\
\hline
\end{tabular}


$\begin{array}{ll}\text { Final } \mathrm{R} \text { indexes [all data] } & \mathrm{R}_{1}=0.0639, \mathrm{wR}_{2}=0.1490 \\ \text { Largest diff. peak/hole / e } \AA^{-3} & 1.74 /-1.31\end{array}$

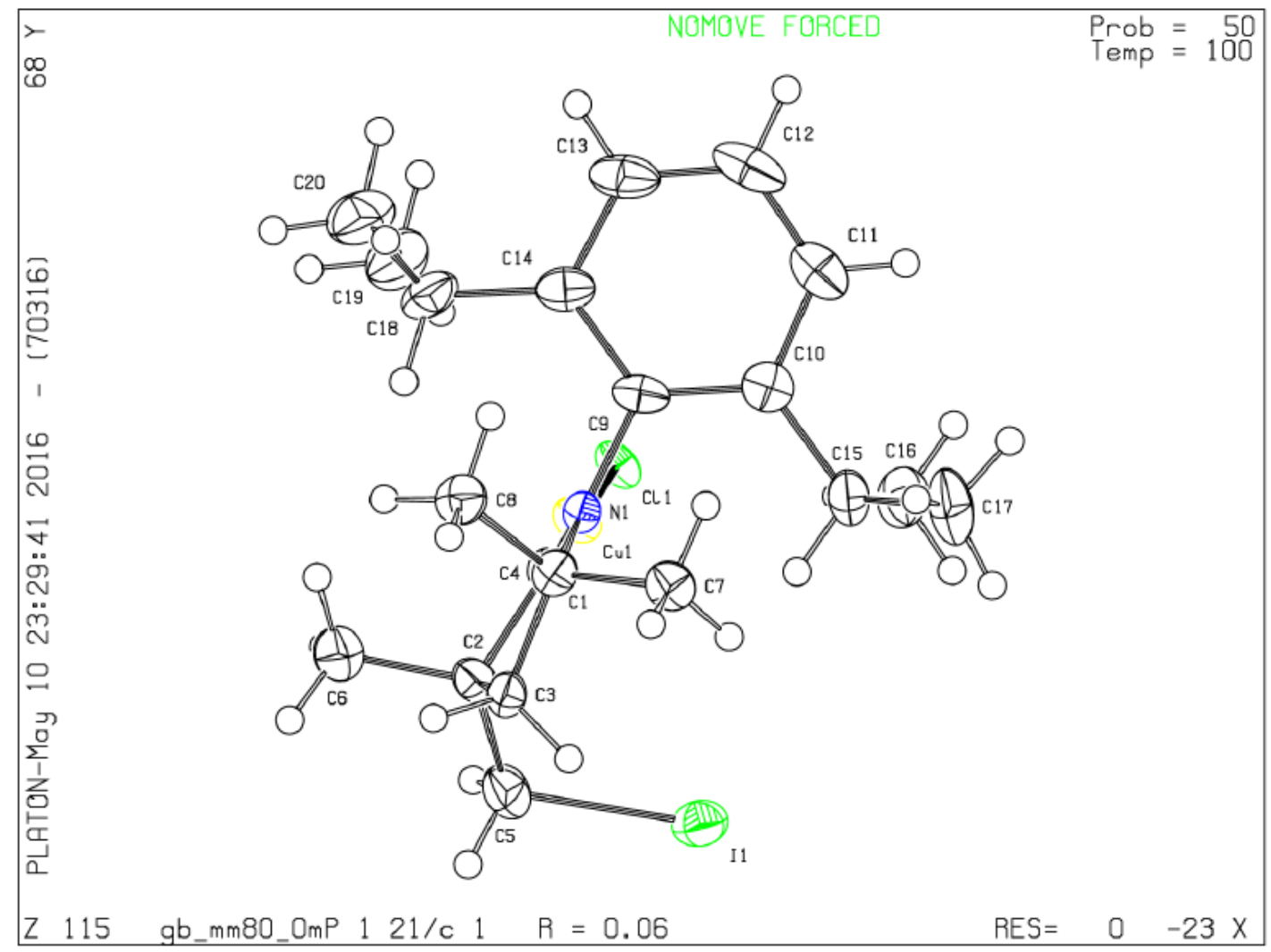

Table S6.3.2. Bond lengths for (6f)CuCl.

\begin{tabular}{|c|c|c|c|c|c|}
\hline Atom & Atom & Length/Å & Atom & Atom & Length $/ \AA ̊$ \\
\hline I1 & $\mathrm{C} 5$ & $2.159(8)$ & $\mathrm{C} 8$ & $\mathrm{H} 8 \mathrm{C}$ & 0.98 \\
\hline $\mathrm{Cu} 1$ & $\mathrm{Cl1}$ & $2.1395(17)$ & $\mathrm{C} 15$ & H15 & 1 \\
\hline $\mathrm{Cu} 1$ & $\mathrm{C} 1$ & $1.872(7)$ & $\mathrm{C} 15$ & $\mathrm{C} 16$ & $1.527(11)$ \\
\hline $\mathrm{N} 1$ & $\mathrm{C} 1$ & $1.314(9)$ & $\mathrm{C} 15$ & $\mathrm{C} 17$ & $1.541(11)$ \\
\hline N1 & C9 & $1.454(9)$ & $\mathrm{C} 6$ & H6A & 0.98 \\
\hline N1 & $\mathrm{C} 4$ & $1.527(9)$ & $\mathrm{C} 6$ & H6B & 0.98 \\
\hline $\mathrm{C} 1$ & $\mathrm{C} 2$ & $1.515(10)$ & $\mathrm{C} 6$ & $\mathrm{H} 6 \mathrm{C}$ & 0.98 \\
\hline $\mathrm{C} 3$ & $\mathrm{H} 3 \mathrm{~A}$ & 0.99 & $\mathrm{C} 13$ & $\mathrm{H} 13$ & 0.95 \\
\hline $\mathrm{C} 3$ & H3B & 0.99 & $\mathrm{C} 13$ & $\mathrm{C} 12$ & $1.376(13)$ \\
\hline $\mathrm{C} 3$ & $\mathrm{C} 4$ & $1.521(10)$ & $\mathrm{C} 12$ & $\mathrm{H} 12$ & 0.95 \\
\hline $\mathrm{C} 3$ & $\mathrm{C} 2$ & $1.543(10)$ & $\mathrm{C} 18$ & $\mathrm{H} 18$ & 1 \\
\hline C9 & $\mathrm{C} 10$ & $1.400(11)$ & $\mathrm{C} 18$ & $\mathrm{C} 19$ & $1.529(12)$ \\
\hline C9 & $\mathrm{C} 14$ & $1.422(11)$ & $\mathrm{C} 18$ & $\mathrm{C} 20$ & $1.534(13)$ \\
\hline $\mathrm{C} 7$ & $\mathrm{H} 7 \mathrm{~A}$ & 0.98 & $\mathrm{C} 5$ & $\mathrm{H} 5 \mathrm{~A}$ & 0.99 \\
\hline $\mathrm{C} 7$ & H7B & 0.98 & $\mathrm{C} 5$ & $\mathrm{H} 5 \mathrm{~B}$ & 0.99 \\
\hline $\mathrm{C} 7$ & $\mathrm{H} 7 \mathrm{C}$ & 0.98 & $\mathrm{C} 16$ & H16A & 0.98 \\
\hline $\mathrm{C} 7$ & $\mathrm{C} 4$ & $1.526(10)$ & $\mathrm{C} 16$ & H16B & 0.98 \\
\hline $\mathrm{C} 4$ & $\mathrm{C} 8$ & $1.526(10)$ & $\mathrm{C} 16$ & $\mathrm{H} 16 \mathrm{C}$ & 0.98 \\
\hline
\end{tabular}




$\begin{array}{cccccc}\text { C10 } & \text { C11 } & 1.397(11) & \text { C19 } & \text { H19A } & 0.98 \\ \text { C10 } & \text { C15 } & 1.521(11) & \text { C19 } & \text { H19B } & 0.98 \\ \text { C2 } & \text { C6 } & 1.550(11) & \text { C19 } & \text { H19C } & 0.98 \\ \text { C2 } & \text { C5 } & 1.523(11) & \text { C17 } & \text { H17A } & 0.98 \\ \text { C14 } & \text { C13 } & 1.399(11) & \text { C17 } & \text { H17B } & 0.98 \\ \text { C14 } & \text { C18 } & 1.506(12) & \text { C17 } & \text { H17C } & 0.98 \\ \text { C11 } & \text { H11 } & 0.95 & \text { C20 } & \text { H20A } & 0.98 \\ \text { C11 } & \text { C12 } & 1.378(13) & \text { C20 } & \text { H20B } & 0.98 \\ \text { C8 } & \text { H8A } & 0.98 & \text { C20 } & \text { H20C } & 0.98 \\ \text { C8 } & \text { H8B } & 0.98 & & & \end{array}$

Table S6.3.3. Bond angles for (6f) CuCl.

\begin{tabular}{|c|c|c|c|c|c|c|c|}
\hline \multicolumn{7}{|c|}{ Angle } & \multirow{2}{*}{$\begin{array}{c}\text { Angle } \\
/ 0\end{array}$} \\
\hline Atom & Atom & Atom & 10 & Atom & Atom & Atom & \\
\hline $\mathrm{C} 1$ & $\mathrm{Cu} 1$ & $\mathrm{C} 11$ & $178.1(2)$ & $\mathrm{C} 16$ & $\mathrm{C} 15$ & H15 & 107.8 \\
\hline $\mathrm{C} 1$ & N1 & C9 & $121.5(6)$ & $\mathrm{C} 16$ & $\mathrm{C} 15$ & $\mathrm{C} 17$ & $110.2(7)$ \\
\hline $\mathrm{C} 1$ & N1 & $\mathrm{C} 4$ & $116.9(6)$ & $\mathrm{C} 17$ & $\mathrm{C} 15$ & H15 & 107.8 \\
\hline C9 & N1 & $\mathrm{C} 4$ & $121.5(5)$ & $\mathrm{C} 2$ & C6 & H6A & 109.5 \\
\hline N1 & $\mathrm{C} 1$ & $\mathrm{Cu} 1$ & $125.0(5)$ & $\mathrm{C} 2$ & C6 & H6B & 109.5 \\
\hline N1 & $\mathrm{C} 1$ & $\mathrm{C} 2$ & $107.7(6)$ & $\mathrm{C} 2$ & C6 & $\mathrm{H} 6 \mathrm{C}$ & 109.5 \\
\hline $\mathrm{C} 2$ & $\mathrm{C} 1$ & $\mathrm{Cu} 1$ & $127.3(5)$ & $\mathrm{H} 6 \mathrm{~A}$ & C6 & H6B & 109.5 \\
\hline $\mathrm{H} 3 \mathrm{~A}$ & $\mathrm{C} 3$ & $\mathrm{H} 3 \mathrm{~B}$ & 108.5 & $\mathrm{H} 6 \mathrm{~A}$ & $\mathrm{C} 6$ & $\mathrm{H} 6 \mathrm{C}$ & 109.5 \\
\hline $\mathrm{C} 4$ & $\mathrm{C} 3$ & $\mathrm{H} 3 \mathrm{~A}$ & 110.2 & H6B & C6 & $\mathrm{H} 6 \mathrm{C}$ & 109.5 \\
\hline $\mathrm{C} 4$ & $\mathrm{C} 3$ & $\mathrm{H} 3 \mathrm{~B}$ & 110.2 & $\mathrm{C} 14$ & $\mathrm{C} 13$ & H13 & 119 \\
\hline $\mathrm{C} 4$ & $\mathrm{C} 3$ & $\mathrm{C} 2$ & $107.6(6)$ & $\mathrm{C} 12$ & $\mathrm{C} 13$ & $\mathrm{C} 14$ & $122.0(8)$ \\
\hline $\mathrm{C} 2$ & $\mathrm{C} 3$ & $\mathrm{H} 3 \mathrm{~A}$ & 110.2 & $\mathrm{C} 12$ & $\mathrm{C} 13$ & H13 & 119 \\
\hline $\mathrm{C} 2$ & $\mathrm{C} 3$ & H3B & 110.2 & $\mathrm{C} 11$ & $\mathrm{C} 12$ & H12 & 120.1 \\
\hline $\mathrm{C} 10$ & C9 & N1 & $119.9(6)$ & $\mathrm{C} 13$ & $\mathrm{C} 12$ & $\mathrm{C} 11$ & $119.8(8)$ \\
\hline $\mathrm{C} 10$ & C9 & $\mathrm{C} 14$ & $122.4(7)$ & $\mathrm{C} 13$ & $\mathrm{C} 12$ & H12 & 120.1 \\
\hline $\mathrm{C} 14$ & C9 & N1 & $117.7(7)$ & $\mathrm{C} 14$ & $\mathrm{C} 18$ & H1 8 & 107.7 \\
\hline $\mathrm{H} 7 \mathrm{~A}$ & $\mathrm{C} 7$ & $\mathrm{H} 7 \mathrm{~B}$ & 109.5 & $\mathrm{C} 14$ & $\mathrm{C} 18$ & C19 & $109.4(7)$ \\
\hline $\mathrm{H} 7 \mathrm{~A}$ & C7 & $\mathrm{H} 7 \mathrm{C}$ & 109.5 & C14 & $\mathrm{C} 18$ & $\mathrm{C} 20$ & $112.8(8)$ \\
\hline H7B & $\mathrm{C} 7$ & $\mathrm{H} 7 \mathrm{C}$ & 109.5 & $\mathrm{C} 19$ & $\mathrm{C} 18$ & H1 8 & 107.7 \\
\hline $\mathrm{C} 4$ & $\mathrm{C} 7$ & $\mathrm{H} 7 \mathrm{~A}$ & 109.5 & $\mathrm{C} 19$ & $\mathrm{C} 18$ & $\mathrm{C} 20$ & $111.2(8)$ \\
\hline $\mathrm{C} 4$ & $\mathrm{C} 7$ & $\mathrm{H} 7 \mathrm{~B}$ & 109.5 & $\mathrm{C} 20$ & $\mathrm{C} 18$ & H18 & 107.7 \\
\hline $\mathrm{C} 4$ & $\mathrm{C} 7$ & $\mathrm{H} 7 \mathrm{C}$ & 109.5 & I1 & $\mathrm{C} 5$ & $\mathrm{H} 5 \mathrm{~A}$ & 108.9 \\
\hline $\mathrm{C} 3$ & $\mathrm{C} 4$ & $\mathrm{~N} 1$ & $99.9(5)$ & I1 & $\mathrm{C} 5$ & H5B & 108.9 \\
\hline $\mathrm{C} 3$ & $\mathrm{C} 4$ & C7 & $112.5(6)$ & $\mathrm{C} 2$ & $\mathrm{C} 5$ & I1 & 113.3(5) \\
\hline $\mathrm{C} 3$ & $\mathrm{C} 4$ & $\mathrm{C} 8$ & $113.2(6)$ & $\mathrm{C} 2$ & $\mathrm{C} 5$ & $\mathrm{H} 5 \mathrm{~A}$ & 108.9 \\
\hline $\mathrm{C} 7$ & $\mathrm{C} 4$ & N1 & $110.9(6)$ & $\mathrm{C} 2$ & $\mathrm{C} 5$ & H5B & 108.9 \\
\hline C7 & $\mathrm{C} 4$ & $\mathrm{C} 8$ & $109.4(6)$ & $\mathrm{H} 5 \mathrm{~A}$ & $\mathrm{C} 5$ & H5B & 107.7 \\
\hline $\mathrm{C} 8$ & $\mathrm{C} 4$ & N1 & $110.6(6)$ & $\mathrm{C} 15$ & C16 & H16A & 109.5 \\
\hline C9 & $\mathrm{C} 10$ & $\mathrm{C} 15$ & $123.1(7)$ & $\mathrm{C} 15$ & $\mathrm{C} 16$ & H16B & 109.5 \\
\hline
\end{tabular}




$\begin{array}{cccccccc}\text { C11 } & \text { C10 } & \text { C9 } & 117.2(7) & \text { C15 } & \text { C16 } & \text { H16C } & 109.5 \\ \text { C11 } & \text { C10 } & \text { C15 } & 119.4(7) & \text { H16A } & \text { C16 } & \text { H16B } & 109.5 \\ \text { C1 } & \text { C2 } & \text { C3 } & 104.1(6) & \text { H16A } & \text { C16 } & \text { H16C } & 109.5 \\ \text { C1 } & \text { C2 } & \text { C6 } & 107.6(6) & \text { H16B } & \text { C16 } & \text { H16C } & 109.5 \\ \text { C1 } & \text { C2 } & \text { C5 } & 111.1(6) & \text { C18 } & \text { C19 } & \text { H19A } & 109.5 \\ \text { C3 } & \text { C2 } & \text { C6 } & 112.9(6) & \text { C18 } & \text { C19 } & \text { H19B } & 109.5 \\ \text { C5 } & \text { C2 } & \text { C3 } & 114.2(6) & \text { C18 } & \text { C19 } & \text { H19C } & 109.5 \\ \text { C5 } & \text { C2 } & \text { C6 } & 106.8(6) & \text { H19A } & \text { C19 } & \text { H19B } & 109.5 \\ \text { C9 } & \text { C14 } & \text { C18 } & 123.3(7) & \text { H19A } & \text { C19 } & \text { H19C } & 109.5 \\ \text { C13 } & \text { C14 } & \text { C9 } & 116.5(8) & \text { H19B } & \text { C19 } & \text { H19C } & 109.5 \\ \text { C13 } & \text { C14 } & \text { C18 } & 119.9(7) & \text { C15 } & \text { C17 } & \text { H17A } & 109.5 \\ \text { C10 } & \text { C11 } & \text { H11 } & 119.1 & \text { C15 } & \text { C17 } & \text { H17B } & 109.5 \\ \text { C12 } & \text { C11 } & \text { C10 } & 121.8(8) & \text { C15 } & \text { C17 } & \text { H17C } & 109.5 \\ \text { C12 } & \text { C11 } & \text { H11 } & 119.1 & \text { H17A } & \text { C17 } & \text { H17B } & 109.5 \\ \text { C4 } & \text { C8 } & \text { H8A } & 109.5 & \text { H17A } & \text { C17 } & \text { H17C } & 109.5 \\ \text { C4 } & \text { C8 } & \text { H8B } & 109.5 & \text { H17B } & \text { C17 } & \text { H17C } & 109.5 \\ \text { C4 } & \text { C8 } & \text { H8C } & 109.5 & \text { C18 } & \text { C20 } & \text { H20A } & 109.5 \\ \text { H8A } & \text { C8 } & \text { H8B } & 109.5 & \text { C18 } & \text { C20 } & \text { H20B } & 109.5 \\ \text { H8A } & \text { C8 } & \text { H8C } & 109.5 & \text { C18 } & \text { C20 } & \text { H20C } & 109.5 \\ \text { H8B } & \text { C8 } & \text { H8C } & 109.5 & \text { H20A } & \text { C20 } & \text { H20B } & 109.5 \\ \text { C10 } & \text { C15 } & \text { H15 } & 107.8 & \text { H20A } & \text { C20 } & \text { H20C } & 109.5 \\ \text { C10 } & \text { C15 } & \text { C16 } & 109.9(7) & \text { H20B } & \text { C20 } & \text { H20C } & 109.5 \\ \text { C10 } & \text { C15 } & \text { C17 } & 113.3(7) & & & & \\ & & & & & & & \end{array}$

Table S6.3.4. Torsion angles for (6f) CuCl.

$\begin{array}{ccccc}\text { Atom } & \text { Atom } & \text { Atom } & \text { Atom } & \text { Torsion Angle }{ }^{\circ} \\ \mathrm{C} 1 & \mathrm{C} 1 & \mathrm{C} 2 & \mathrm{C} 3 & 166.3(5) \\ \mathrm{C} 11 & \mathrm{C} 1 & \mathrm{C} 2 & \mathrm{C} 6 & -73.7(8) \\ \mathrm{C} 11 & \mathrm{C} 1 & \mathrm{C} 2 & \mathrm{C} 5 & 42.9(9) \\ \mathrm{N} 1 & \mathrm{C} 1 & \mathrm{C} 2 & \mathrm{C} 3 & -15.5(8) \\ \mathrm{N} 1 & \mathrm{C} 1 & \mathrm{C} 2 & \mathrm{C} 6 & 104.5(7) \\ \mathrm{N} 1 & \mathrm{C} 1 & \mathrm{C} 2 & \mathrm{C} 5 & -138.9(6) \\ \mathrm{N} 1 & \mathrm{C} 9 & \mathrm{C} 10 & \mathrm{C} 11 & -177.2(6) \\ \mathrm{N} 1 & \mathrm{C} 9 & \mathrm{C} 10 & \mathrm{C} 15 & 8.8(10) \\ \mathrm{N} 1 & \mathrm{C} 9 & \mathrm{C} 14 & \mathrm{C} 13 & 178.2(6) \\ \mathrm{N} 1 & \mathrm{C} 9 & \mathrm{C} 14 & \mathrm{C} 18 & -9.0(10) \\ \mathrm{C} 1 & \mathrm{~N} 1 & \mathrm{C} 9 & \mathrm{C} 10 & -91.8(8) \\ \mathrm{C} 1 & \mathrm{~N} 1 & \mathrm{C} 9 & \mathrm{C} 14 & 86.2(8) \\ \mathrm{C} 1 & \mathrm{~N} 1 & \mathrm{C} 4 & \mathrm{C} 3 & 6.8(8) \\ \mathrm{C} 1 & \mathrm{~N} 1 & \mathrm{C} 4 & \mathrm{C} 7 & 125.7(7) \\ \mathrm{C} 1 & \mathrm{~N} 1 & \mathrm{C} 4 & \mathrm{C} 8 & -112.8(7) \\ \mathrm{C} 1 & \mathrm{C} 2 & \mathrm{C} 5 & \mathrm{I} 1 & 50.4(8) \\ \mathrm{C} 3 & \mathrm{C} 2 & \mathrm{C} 5 & \mathrm{I} 1 & -67.0(7)\end{array}$




\begin{tabular}{|c|c|c|c|c|}
\hline C9 & $\mathrm{N} 1$ & $\mathrm{C} 1$ & $\mathrm{Cu} 1$ & $5.3(9)$ \\
\hline C9 & N1 & $\mathrm{C} 1$ & $\mathrm{C} 2$ & $-172.9(6)$ \\
\hline C9 & N1 & $\mathrm{C} 4$ & $\mathrm{C} 3$ & $-174.6(6)$ \\
\hline C9 & N1 & $\mathrm{C} 4$ & $\mathrm{C} 7$ & $-55.8(8)$ \\
\hline C9 & $\mathrm{N} 1$ & $\mathrm{C} 4$ & $\mathrm{C} 8$ & $65.8(8)$ \\
\hline $\mathrm{C} 9$ & $\mathrm{C} 10$ & $\mathrm{C} 11$ & $\mathrm{C} 12$ & $-2.4(11)$ \\
\hline C9 & $\mathrm{C} 10$ & $\mathrm{C} 15$ & $\mathrm{C} 16$ & 107.9(8) \\
\hline C9 & $\mathrm{C} 10$ & $\mathrm{C} 15$ & $\mathrm{C} 17$ & $-128.4(8)$ \\
\hline C9 & $\mathrm{C} 14$ & $\mathrm{C} 13$ & $\mathrm{C} 12$ & $0.4(12)$ \\
\hline $\mathrm{C} 9$ & $\mathrm{C} 14$ & $\mathrm{C} 18$ & $\mathrm{C} 19$ & $-104.5(9)$ \\
\hline $\mathrm{C} 9$ & $\mathrm{C} 14$ & $\mathrm{C} 18$ & $\mathrm{C} 20$ & 131.1(8) \\
\hline $\mathrm{C} 4$ & $\mathrm{~N} 1$ & $\mathrm{C} 1$ & $\mathrm{Cu} 1$ & $-176.1(5)$ \\
\hline $\mathrm{C} 4$ & $\mathrm{~N} 1$ & $\mathrm{C} 1$ & $\mathrm{C} 2$ & $5.6(8)$ \\
\hline $\mathrm{C} 4$ & $\mathrm{~N} 1$ & $\mathrm{C} 9$ & $\mathrm{C} 10$ & $89.7(8)$ \\
\hline $\mathrm{C} 4$ & N1 & $\mathrm{C} 9$ & $\mathrm{C} 14$ & $-92.3(8)$ \\
\hline $\mathrm{C} 4$ & $\mathrm{C} 3$ & $\mathrm{C} 2$ & $\mathrm{C} 1$ & $19.8(7)$ \\
\hline $\mathrm{C} 4$ & $\mathrm{C} 3$ & $\mathrm{C} 2$ & $\mathrm{C} 6$ & $-96.5(7)$ \\
\hline $\mathrm{C} 4$ & $\mathrm{C} 3$ & $\mathrm{C} 2$ & $\mathrm{C} 5$ & $141.2(6)$ \\
\hline $\mathrm{C} 10$ & C9 & $\mathrm{C} 14$ & $\mathrm{C} 13$ & $-3.9(11)$ \\
\hline $\mathrm{C} 10$ & $\mathrm{C} 9$ & $\mathrm{C} 14$ & $\mathrm{C} 18$ & $169.0(7)$ \\
\hline $\mathrm{C} 10$ & $\mathrm{C} 11$ & $\mathrm{C} 12$ & $\mathrm{C} 13$ & $-0.9(12)$ \\
\hline $\mathrm{C} 2$ & $\mathrm{C} 3$ & $\mathrm{C} 4$ & N1 & $-16.0(7)$ \\
\hline $\mathrm{C} 2$ & $\mathrm{C} 3$ & $\mathrm{C} 4$ & $\mathrm{C} 7$ & $-133.7(6)$ \\
\hline $\mathrm{C} 2$ & $\mathrm{C} 3$ & $\mathrm{C} 4$ & $\mathrm{C} 8$ & $101.6(7)$ \\
\hline $\mathrm{C} 14$ & $\mathrm{C} 9$ & $\mathrm{C} 10$ & $\mathrm{C} 11$ & $4.9(10)$ \\
\hline C14 & $\mathrm{C} 9$ & $\mathrm{C} 10$ & $\mathrm{C} 15$ & $-169.1(7)$ \\
\hline $\mathrm{C} 14$ & $\mathrm{C} 13$ & $\mathrm{C} 12$ & $\mathrm{C} 11$ & $1.9(13)$ \\
\hline $\mathrm{C} 11$ & $\mathrm{C} 10$ & $\mathrm{C} 15$ & $\mathrm{C} 16$ & $-66.0(9)$ \\
\hline $\mathrm{C} 11$ & $\mathrm{C} 10$ & $\mathrm{C} 15$ & $\mathrm{C} 17$ & $57.7(9)$ \\
\hline $\mathrm{C} 15$ & $\mathrm{C} 10$ & $\mathrm{C} 11$ & $\mathrm{C} 12$ & $171.8(7)$ \\
\hline $\mathrm{C} 6$ & $\mathrm{C} 2$ & $\mathrm{C} 5$ & I1 & $167.5(5)$ \\
\hline $\mathrm{C} 13$ & $\mathrm{C} 14$ & $\mathrm{C} 18$ & $\mathrm{C} 19$ & $68.2(10)$ \\
\hline $\mathrm{C} 13$ & $\mathrm{C} 14$ & $\mathrm{C} 18$ & $\mathrm{C} 20$ & $-56.3(10)$ \\
\hline $\mathrm{C} 18$ & $\mathrm{C} 14$ & $\mathrm{C} 13$ & $\mathrm{C} 12$ & $-172.7(8)$ \\
\hline
\end{tabular}

\subsection{Gold(III) Biphenylene Complex 7}

Single crystals of $\mathrm{C}_{78} \mathrm{H}_{68} \mathrm{AuBF}_{20} \mathrm{~N}_{2} 7$ were grown by slow evaporation of a mixture of benzene/pentane. A suitable crystal was selected and mounted on a 'Bruker APEX-II CCD' diffractometer. The crystal was kept at $100 \mathrm{~K}$ during data collection. Using Olex $2{ }^{8}$ the structure was solved with the ShelXS ${ }^{9}$ structure solution program using Direct Methods and refined with the ShelXL ${ }^{9}$ refinement package using Least Squares minimization. 
Table S6.4.1. Crystal data and structure refinement for 7.

Identification code

Empirical formula

Formula weight

Temperature/K

Crystal system

Space group

$\mathrm{a} / \AA$

$\mathrm{b} / \AA$

$\mathrm{c} / \AA$

$\alpha /{ }^{\circ}$

$\beta /{ }^{\circ}$

$\gamma /{ }^{\circ}$

Volume $/ \AA^{3}$

$\mathrm{Z}$

$\rho_{\text {calc }} \mathrm{g} / \mathrm{cm}^{3}$

$\mu / \mathrm{mm}^{-1}$

$\mathrm{F}(000)$

Crystal size $/ \mathrm{mm}^{3}$

Radiation

$2 \Theta$ range for data collection $/{ }^{\circ}$

Index ranges

Reflections collected

Independent reflections

Data/restraints/parameters

Goodness-of-fit on $\mathrm{F}^{2}$

Final $\mathrm{R}$ indexes $[\mathrm{I}>=2 \sigma(\mathrm{I})]$

Final R indexes [all data]

Largest diff. peak/hole / e $\AA^{-3}$
7

$\mathrm{C}_{78} \mathrm{H}_{68} \mathrm{AuBF}_{20} \mathrm{~N}_{2}$ 1621.12

100

triclinic

P-1

13.7142(5)

$15.7707(7)$

$15.8385(7)$

$88.698(2)$

$87.417(2)$

$82.569(2)$

$3392.9(2)$

2

1.587

2.270

1628.0

$0.1 \times 0.05 \times 0.05$

$\operatorname{MoK} \alpha(\lambda=0.71073)$

4.418 to 50.904

$-16 \leq \mathrm{h} \leq 16,-19 \leq \mathrm{k} \leq 19,-19 \leq 1 \leq 19$

54719

$12513\left[\mathrm{R}_{\text {int }}=0.0583, \mathrm{R}_{\text {sigma }}=0.0555\right]$

$12513 / 0 / 933$

1.012

$\mathrm{R}_{1}=0.0311, \mathrm{wR}_{2}=0.0586$

$\mathrm{R}_{1}=0.0446, \mathrm{wR}_{2}=0.0627$

$0.73 /-0.78$

Crystal Data for $\mathrm{C}_{78} \mathrm{H}_{68} \mathrm{AuBF}_{20} \mathrm{~N}_{2}(\mathrm{M}=1621.12 \mathrm{~g} / \mathrm{mol})$ : triclinic, space group P-1 (no. 2), $\mathrm{a}=$ 13.7142(5) $\AA, \mathrm{b}=15.7707(7) \AA, \mathrm{c}=15.8385(7) \AA, \alpha=88.698(2)^{\circ}, \beta=87.417(2)^{\circ}, \gamma=$ $82.569(2)^{\circ}, \mathrm{V}=3392.9(2) \AA 3, \mathrm{Z}=2, \mathrm{~T}=100 \mathrm{~K}, \mu(\mathrm{MoK} \alpha)=2.270 \mathrm{~mm}-1$, Dcalc $=1.587$ $\mathrm{g} / \mathrm{cm} 3,54719$ reflections measured $\left(4.418^{\circ} \leq 2 \Theta \leq 50.904^{\circ}\right), 12513$ unique (Rint $=0.0583$, Rsigma $=0.0555)$ which were used in all calculations. The final R1 was $0.0311(\mathrm{I}>2 \sigma(\mathrm{I}))$ and wR2 was 0.0627 (all data). 


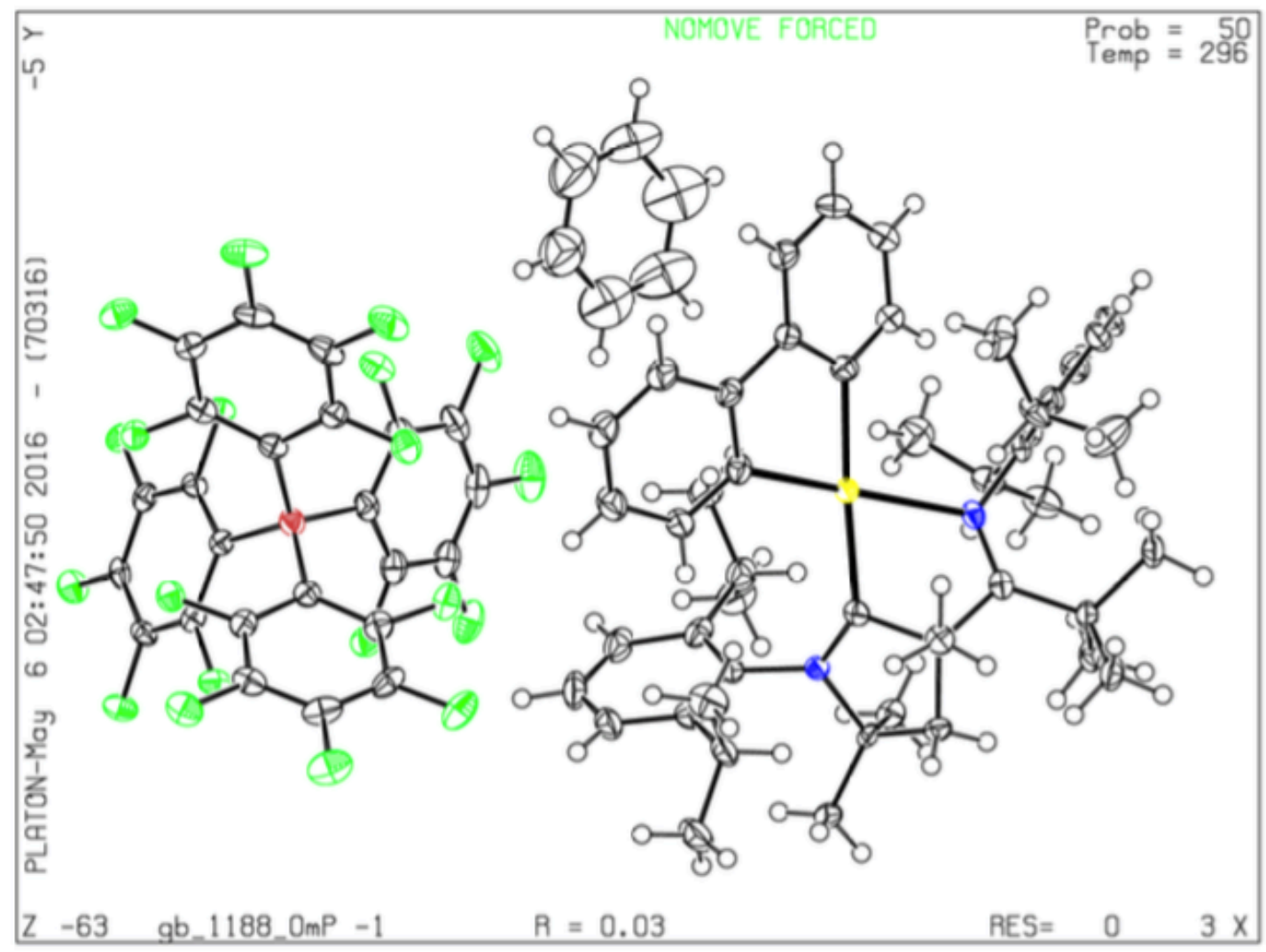

Table S6.4.2. Bond Lengths for 7.

\begin{tabular}{|c|c|c|c|c|c|}
\hline Atom & Atom & Length/Å & Atom & Atom & Length/Å \\
\hline Aul & N1 & $2.246(3)$ & C38 & $\mathrm{C} 46$ & $1.523(5)$ \\
\hline Aul & $\mathrm{C} 13$ & $2.091(3)$ & C38 & C39 & $1.392(5)$ \\
\hline Aul & C12 & $2.051(3)$ & C19A & $\mathrm{C} 20 \mathrm{~A}$ & $1.401(5)$ \\
\hline $\mathrm{Au} 1$ & $\mathrm{C} 1$ & $2.077(3)$ & C19A & B1 & $1.650(5)$ \\
\hline F20 & $\mathrm{C} 24 \mathrm{~A}$ & $1.360(4)$ & C13A & C14A & $1.390(5)$ \\
\hline F7 & C9A & $1.348(4)$ & $\mathrm{C} 13 \mathrm{~A}$ & C18A & $1.396(5)$ \\
\hline F6 & $\mathrm{C} 8 \mathrm{~A}$ & $1.367(3)$ & C13A & B1 & $1.662(5)$ \\
\hline F10 & $\mathrm{C} 12 \mathrm{~A}$ & $1.348(4)$ & $\mathrm{C} 5 \mathrm{~A}$ & C6A & $1.385(5)$ \\
\hline $\mathrm{F} 1$ & $\mathrm{C} 2 \mathrm{~A}$ & $1.347(4)$ & $\mathrm{C} 5 \mathrm{~A}$ & $\mathrm{C} 4 \mathrm{~A}$ & $1.377(5)$ \\
\hline F9 & $\mathrm{C} 11 \mathrm{~A}$ & $1.352(3)$ & $\mathrm{C} 23 \mathrm{~A}$ & $\mathrm{C} 22 \mathrm{~A}$ & $1.376(5)$ \\
\hline F11 & $\mathrm{C} 14 \mathrm{~A}$ & $1.358(4)$ & $\mathrm{C} 22 \mathrm{~A}$ & $\mathrm{C} 21 \mathrm{~A}$ & $1.373(5)$ \\
\hline F16 & $\mathrm{C} 20 \mathrm{~A}$ & $1.353(4)$ & $\mathrm{C} 7$ & $\mathrm{C} 8$ & $1.386(5)$ \\
\hline F8 & $\mathrm{C} 10 \mathrm{~A}$ & $1.340(4)$ & $\mathrm{C} 46$ & C48 & $1.537(5)$ \\
\hline F15 & $\mathrm{C} 18 \mathrm{~A}$ & $1.354(4)$ & $\mathrm{C} 46$ & $\mathrm{C} 47$ & $1.537(5)$ \\
\hline F5 & C6A & $1.355(4)$ & $\mathrm{C} 30$ & $\mathrm{C} 25$ & $1.396(5)$ \\
\hline F19 & $\mathrm{C} 23 \mathrm{~A}$ & $1.352(4)$ & C30 & C31 & $1.521(5)$ \\
\hline F18 & $\mathrm{C} 22 \mathrm{~A}$ & $1.348(4)$ & C30 & $\mathrm{C} 29$ & $1.399(4)$ \\
\hline F12 & $\mathrm{C} 15 \mathrm{~A}$ & $1.349(4)$ & $\mathrm{C} 25$ & $\mathrm{C} 26$ & $1.403(5)$ \\
\hline $\mathrm{F} 2$ & $\mathrm{C} 3 \mathrm{~A}$ & $1.355(4)$ & $\mathrm{C} 2$ & $\mathrm{C} 3$ & $1.395(5)$ \\
\hline F4 & $\mathrm{C} 5 \mathrm{~A}$ & $1.346(4)$ & $\mathrm{C} 26$ & C34 & $1.522(5)$ \\
\hline F3 & $\mathrm{C} 4 \mathrm{~A}$ & $1.352(4)$ & $\mathrm{C} 26$ & $\mathrm{C} 27$ & $1.400(4)$ \\
\hline
\end{tabular}




$\begin{array}{llllll}\mathrm{F} 14 & \mathrm{C} 17 \mathrm{~A} & 1.348(4) & \mathrm{C} 7 \mathrm{~A} & \mathrm{C} 8 \mathrm{~A} & 1.379(5) \\ \mathrm{F} 17 & \mathrm{C} 21 \mathrm{~A} & 1.349(4) & \mathrm{C} 7 \mathrm{~A} & \mathrm{~B} 1 & 1.653(5) \\ \mathrm{F} 13 & \mathrm{C} 16 \mathrm{~A} & 1.352(4) & \mathrm{C} 20 \mathrm{~A} & \mathrm{C} 21 \mathrm{~A} & 1.373(5) \\ \mathrm{N} 2 & \mathrm{C} 13 & 1.296(4) & \mathrm{C} 6 \mathrm{~A} & \mathrm{C} 1 \mathrm{~A} & 1.382(5) \\ \mathrm{N} 2 & \mathrm{C} 16 & 1.531(4) & \mathrm{C} 42 & \mathrm{C} 43 & 1.517(5) \\ \mathrm{N} 2 & \mathrm{C} 37 & 1.464(4) & \mathrm{C} 42 & \mathrm{C} 41 & 1.398(5) \\ \mathrm{N} 1 & \mathrm{C} 20 & 1.288(4) & \mathrm{C} 14 \mathrm{~A} & \mathrm{C} 15 \mathrm{~A} & 1.382(5) \\ \mathrm{N} 1 & \mathrm{C} 25 & 1.451(4) & \mathrm{C} 4 \mathrm{~A} & \mathrm{C} 3 \mathrm{~A} & 1.373(5) \\ \mathrm{C} 13 & \mathrm{C} 14 & 1.519(5) & \mathrm{C} 31 & \mathrm{C} 33 & 1.534(5) \\ \mathrm{C} 14 & \mathrm{C} 20 & 1.545(4) & \mathrm{C} 31 & \mathrm{C} 32 & 1.536(5) \\ \mathrm{C} 14 & \mathrm{C} 15 & 1.551(4) & \mathrm{C} 1 \mathrm{~A} & \mathrm{C} 2 \mathrm{~A} & 1.400(4) \\ \mathrm{C} 14 & \mathrm{C} 19 & 1.559(4) & \mathrm{C} 1 \mathrm{~A} & \mathrm{~B} 1 & 1.652(5) \\ \mathrm{C} 16 & \mathrm{C} 18 & 1.526(4) & \mathrm{C} 29 & \mathrm{C} 28 & 1.381(5) \\ \mathrm{C} 16 & \mathrm{C} 15 & 1.527(4) & \mathrm{C} 34 & \mathrm{C} 35 & 1.530(5) \\ \mathrm{C} 16 & \mathrm{C} 17 & 1.524(4) & \mathrm{C} 34 & \mathrm{C} 36 & 1.522(5) \\ \mathrm{C} 12 & \mathrm{C} 11 & 1.372(5) & \mathrm{C} 27 & \mathrm{C} 28 & 1.375(5) \\ \mathrm{C} 12 & \mathrm{C} 7 & 1.400(5) & \mathrm{C} 10 & \mathrm{C} 9 & 1.381(5) \\ \mathrm{C} 11 \mathrm{~A} & \mathrm{C} 12 \mathrm{~A} & 1.382(5) & \mathrm{C} 18 \mathrm{~A} & \mathrm{C} 17 \mathrm{~A} & 1.384(5) \\ \mathrm{C} 11 \mathrm{~A} & \mathrm{C} 10 \mathrm{~A} & 1.369(5) & \mathrm{C} 3 \mathrm{~A} & \mathrm{C} 2 \mathrm{~A} & 1.371(5) \\ \mathrm{C} 20 & \mathrm{C} 21 & 1.559(5) & \mathrm{C} 43 & \mathrm{C} 44 & 1.533(5) \\ \mathrm{C} 21 & \mathrm{C} 22 & 1.555(5) & \mathrm{C} 43 & \mathrm{C} 45 & 1.539(5) \\ \mathrm{C} 21 & \mathrm{C} 23 & 1.537(5) & \mathrm{C} 5 & \mathrm{C} 4 & 1.386(5) \\ \mathrm{C} 21 & \mathrm{C} 24 & 1.553(4) & \mathrm{C} 41 & \mathrm{C} 40 & 1.379(6) \\ \mathrm{C} 1 & \mathrm{C} 6 & 1.407(5) & \mathrm{C} 15 \mathrm{~A} & \mathrm{C} 16 \mathrm{~A} & 1.366(5) \\ \mathrm{C} 1 & \mathrm{C} 2 & 1.386(5) & \mathrm{C} 9 & \mathrm{C} 8 & 1.386(5) \\ \mathrm{C} 37 & \mathrm{C} 38 & 1.392(5) & \mathrm{C} 16 \mathrm{~A} & \mathrm{C} 17 \mathrm{~A} & 1.365(5) \\ \mathrm{C} 37 & \mathrm{C} 42 & 1.418(5) & \mathrm{C} 3 & \mathrm{C} 4 & 1.371(5) \\ \mathrm{C} 24 \mathrm{~A} & \mathrm{C} 19 \mathrm{~A} & 1.375(5) & \mathrm{C} 39 & \mathrm{C} 40 & 1.376(5) \\ \mathrm{C} 24 \mathrm{~A} & \mathrm{C} 23 \mathrm{~A} & 1.386(5) & \mathrm{C} 2 \mathrm{~B} & \mathrm{C} 3 \mathrm{~B} & 1.391(8) \\ \mathrm{C} 11 & \mathrm{C} 10 & 1.396(5) & \mathrm{C} 2 \mathrm{~B} & \mathrm{C} 1 \mathrm{~B} & 1.366(7) \\ \mathrm{C} 9 \mathrm{~A} & \mathrm{C} 10 \mathrm{~A} & 1.383(4) & \mathrm{C} 3 \mathrm{~B} & \mathrm{C} 4 \mathrm{~B} & 1.388(8) \\ \mathrm{C} 9 \mathrm{~A} & \mathrm{C} 8 \mathrm{~A} & 1.372(5) & \mathrm{C} 4 \mathrm{~B} & \mathrm{C} 5 \mathrm{~B} & 1.350(7) \\ \mathrm{C} 12 \mathrm{~A} & \mathrm{C} 7 \mathrm{~A} & 1.401(4) & \mathrm{C} 5 \mathrm{~B} & \mathrm{C} 6 \mathrm{~B} & 1.373(8) \\ \mathrm{C} 6 & \mathrm{C} 7 & 1.467(5) & \mathrm{C} 6 \mathrm{~B} & \mathrm{C} 1 \mathrm{~B} & 1.387(8) \\ \mathrm{C} 6 & \mathrm{C} 5 & 1.388(5) & & & \\ & & & & & \\ & & & & \end{array}$

Table S6.4.3. Bond Angles for 7.

\begin{tabular}{|c|c|c|c|c|c|c|c|}
\hline Atom & Atom & Atom & Angle ${ }^{\circ}$ & Atom & Atom & Atom & Angle $/^{\circ}$ \\
\hline $\mathrm{C} 13$ & Aul & N1 & $73.76(11)$ & $\mathrm{C} 25$ & $\mathrm{C} 30$ & $\mathrm{C} 31$ & $123.3(3)$ \\
\hline $\mathrm{C} 12$ & $\mathrm{Au} 1$ & N1 & $168.71(12)$ & $\mathrm{C} 25$ & $\mathrm{C} 30$ & $\mathrm{C} 29$ & $116.6(3)$ \\
\hline $\mathrm{C} 12$ & $\mathrm{Au} 1$ & $\mathrm{C} 13$ & $106.36(13)$ & $\mathrm{C} 29$ & $\mathrm{C} 30$ & $\mathrm{C} 31$ & $120.0(3)$ \\
\hline $\mathrm{C} 12$ & $\mathrm{Au} 1$ & $\mathrm{C} 1$ & $78.94(14)$ & $\mathrm{C} 30$ & $\mathrm{C} 25$ & N1 & $117.5(3)$ \\
\hline $\mathrm{C} 1$ & Aul & N1 & $101.63(12)$ & $\mathrm{C} 30$ & $\mathrm{C} 25$ & $\mathrm{C} 26$ & $124.2(3)$ \\
\hline
\end{tabular}




\begin{tabular}{|c|c|c|c|c|c|c|c|}
\hline $\mathrm{C} 1$ & Au1 & $\mathrm{C} 13$ & $173.98(13)$ & $\mathrm{C} 26$ & $\mathrm{C} 25$ & N1 & $118.2(3)$ \\
\hline $\mathrm{C} 13$ & $\mathrm{~N} 2$ & $\mathrm{C} 16$ & $113.9(3)$ & $\mathrm{C} 1$ & $\mathrm{C} 2$ & $\mathrm{C} 3$ & $120.1(3)$ \\
\hline $\mathrm{C} 13$ & $\mathrm{~N} 2$ & $\mathrm{C} 37$ & $127.3(3)$ & $\mathrm{C} 25$ & $\mathrm{C} 26$ & $\mathrm{C} 34$ & $124.1(3)$ \\
\hline C37 & $\mathrm{N} 2$ & $\mathrm{C} 16$ & $118.8(2)$ & $\mathrm{C} 27$ & $\mathrm{C} 26$ & $\mathrm{C} 25$ & 116.1(3) \\
\hline $\mathrm{C} 20$ & $\mathrm{~N} 1$ & $\mathrm{Au} 1$ & $119.5(2)$ & $\mathrm{C} 27$ & $\mathrm{C} 26$ & $\mathrm{C} 34$ & $119.7(3)$ \\
\hline $\mathrm{C} 20$ & N1 & $\mathrm{C} 25$ & $123.3(3)$ & $\mathrm{C} 12 \mathrm{~A}$ & $\mathrm{C} 7 \mathrm{~A}$ & B1 & $126.2(3)$ \\
\hline $\mathrm{C} 25$ & $\mathrm{~N} 1$ & $\mathrm{Au} 1$ & $117.0(2)$ & $\mathrm{C} 8 \mathrm{~A}$ & $\mathrm{C} 7 \mathrm{~A}$ & $\mathrm{C} 12 \mathrm{~A}$ & $112.8(3)$ \\
\hline N2 & $\mathrm{C} 13$ & Aul & $135.4(3)$ & $\mathrm{C} 8 \mathrm{~A}$ & $\mathrm{C} 7 \mathrm{~A}$ & B1 & $120.2(3)$ \\
\hline N2 & $\mathrm{C} 13$ & $\mathrm{C} 14$ & $109.4(3)$ & F16 & $\mathrm{C} 20 \mathrm{~A}$ & C19A & $119.0(3)$ \\
\hline $\mathrm{C} 14$ & $\mathrm{C} 13$ & $\mathrm{Au} 1$ & $113.1(2)$ & F16 & $\mathrm{C} 20 \mathrm{~A}$ & $\mathrm{C} 21 \mathrm{~A}$ & $116.0(3)$ \\
\hline $\mathrm{C} 13$ & $\mathrm{C} 14$ & $\mathrm{C} 20$ & $109.5(3)$ & $\mathrm{C} 21 \mathrm{~A}$ & $\mathrm{C} 20 \mathrm{~A}$ & C19A & $124.9(4)$ \\
\hline $\mathrm{C} 13$ & $\mathrm{C} 14$ & $\mathrm{C} 15$ & $103.9(3)$ & F5 & C6A & $\mathrm{C} 5 \mathrm{~A}$ & $115.0(3)$ \\
\hline $\mathrm{C} 13$ & $\mathrm{C} 14$ & $\mathrm{C} 19$ & $103.3(3)$ & F5 & C6A & $\mathrm{C} 1 \mathrm{~A}$ & $120.5(3)$ \\
\hline $\mathrm{C} 20$ & $\mathrm{C} 14$ & $\mathrm{C} 15$ & $120.2(3)$ & $\mathrm{C} 1 \mathrm{~A}$ & C6A & $\mathrm{C} 5 \mathrm{~A}$ & $124.5(3)$ \\
\hline $\mathrm{C} 20$ & $\mathrm{C} 14$ & $\mathrm{C} 19$ & 107.1(3) & $\mathrm{C} 37$ & $\mathrm{C} 42$ & $\mathrm{C} 43$ & $124.0(3)$ \\
\hline $\mathrm{C} 15$ & $\mathrm{C} 14$ & $\mathrm{C} 19$ & $111.5(3)$ & $\mathrm{C} 41$ & $\mathrm{C} 42$ & $\mathrm{C} 37$ & $115.9(3)$ \\
\hline $\mathrm{C} 18$ & $\mathrm{C} 16$ & $\mathrm{~N} 2$ & $106.8(3)$ & $\mathrm{C} 41$ & $\mathrm{C} 42$ & $\mathrm{C} 43$ & $120.0(3)$ \\
\hline $\mathrm{C} 18$ & $\mathrm{C} 16$ & $\mathrm{C} 15$ & $112.6(3)$ & F11 & $\mathrm{C} 14 \mathrm{~A}$ & $\mathrm{C} 13 \mathrm{~A}$ & $119.2(3)$ \\
\hline $\mathrm{C} 15$ & $\mathrm{C} 16$ & $\mathrm{~N} 2$ & $99.8(2)$ & F11 & $\mathrm{C} 14 \mathrm{~A}$ & $\mathrm{C} 15 \mathrm{~A}$ & $115.6(3)$ \\
\hline $\mathrm{C} 17$ & $\mathrm{C} 16$ & $\mathrm{~N} 2$ & $114.6(3)$ & $\mathrm{C} 15 \mathrm{~A}$ & $\mathrm{C} 14 \mathrm{~A}$ & $\mathrm{C} 13 \mathrm{~A}$ & $125.2(3)$ \\
\hline $\mathrm{C} 17$ & $\mathrm{C} 16$ & $\mathrm{C} 18$ & $108.9(3)$ & F3 & $\mathrm{C} 4 \mathrm{~A}$ & $\mathrm{C} 5 \mathrm{~A}$ & $120.0(4)$ \\
\hline $\mathrm{C} 17$ & $\mathrm{C} 16$ & $\mathrm{C} 15$ & $113.7(3)$ & F3 & $\mathrm{C} 4 \mathrm{~A}$ & $\mathrm{C} 3 \mathrm{~A}$ & $120.9(3)$ \\
\hline $\mathrm{C} 11$ & $\mathrm{C} 12$ & $\mathrm{Au} 1$ & $125.5(3)$ & $\mathrm{C} 3 \mathrm{~A}$ & $\mathrm{C} 4 \mathrm{~A}$ & $\mathrm{C} 5 \mathrm{~A}$ & $119.2(3)$ \\
\hline $\mathrm{C} 11$ & $\mathrm{C} 12$ & $\mathrm{C} 7$ & $120.1(3)$ & $\mathrm{C} 30$ & $\mathrm{C} 31$ & $\mathrm{C} 33$ & $113.2(3)$ \\
\hline $\mathrm{C} 7$ & $\mathrm{C} 12$ & $\mathrm{Au} 1$ & $114.3(2)$ & $\mathrm{C} 30$ & $\mathrm{C} 31$ & $\mathrm{C} 32$ & $110.6(3)$ \\
\hline F9 & $\mathrm{C} 11 \mathrm{~A}$ & $\mathrm{C} 12 \mathrm{~A}$ & $120.3(3)$ & $\mathrm{C} 33$ & $\mathrm{C} 31$ & $\mathrm{C} 32$ & $110.3(3)$ \\
\hline F9 & $\mathrm{C} 11 \mathrm{~A}$ & $\mathrm{C} 10 \mathrm{~A}$ & $119.2(3)$ & $\mathrm{C} 6 \mathrm{~A}$ & $\mathrm{C} 1 \mathrm{~A}$ & $\mathrm{C} 2 \mathrm{~A}$ & $113.3(3)$ \\
\hline $\mathrm{C} 10 \mathrm{~A}$ & $\mathrm{C} 11 \mathrm{~A}$ & $\mathrm{C} 12 \mathrm{~A}$ & $120.4(3)$ & $\mathrm{C} 6 \mathrm{~A}$ & $\mathrm{C} 1 \mathrm{~A}$ & B1 & $127.4(3)$ \\
\hline N1 & $\mathrm{C} 20$ & $\mathrm{C} 14$ & $112.5(3)$ & $\mathrm{C} 2 \mathrm{~A}$ & $\mathrm{C} 1 \mathrm{~A}$ & B1 & 119.1(3) \\
\hline N1 & $\mathrm{C} 20$ & $\mathrm{C} 21$ & $128.5(3)$ & $\mathrm{C} 28$ & $\mathrm{C} 29$ & $\mathrm{C} 30$ & $120.7(3)$ \\
\hline $\mathrm{C} 14$ & $\mathrm{C} 20$ & $\mathrm{C} 21$ & $119.0(3)$ & F17 & $\mathrm{C} 21 \mathrm{~A}$ & $\mathrm{C} 22 \mathrm{~A}$ & $120.1(3)$ \\
\hline $\mathrm{C} 22$ & $\mathrm{C} 21$ & $\mathrm{C} 20$ & $108.4(3)$ & F17 & $\mathrm{C} 21 \mathrm{~A}$ & $\mathrm{C} 20 \mathrm{~A}$ & $120.8(4)$ \\
\hline $\mathrm{C} 23$ & $\mathrm{C} 21$ & $\mathrm{C} 20$ & $111.4(3)$ & $\mathrm{C} 20 \mathrm{~A}$ & $\mathrm{C} 21 \mathrm{~A}$ & $\mathrm{C} 22 \mathrm{~A}$ & 119.1(3) \\
\hline $\mathrm{C} 23$ & $\mathrm{C} 21$ & $\mathrm{C} 22$ & $109.7(3)$ & $\mathrm{C} 26$ & $\mathrm{C} 34$ & $\mathrm{C} 35$ & $111.5(3)$ \\
\hline $\mathrm{C} 23$ & $\mathrm{C} 21$ & $\mathrm{C} 24$ & $105.3(3)$ & $\mathrm{C} 26$ & $\mathrm{C} 34$ & $\mathrm{C} 36$ & $113.9(3)$ \\
\hline $\mathrm{C} 24$ & $\mathrm{C} 21$ & $\mathrm{C} 20$ & $116.8(3)$ & $\mathrm{C} 36$ & $\mathrm{C} 34$ & $\mathrm{C} 35$ & $108.7(3)$ \\
\hline $\mathrm{C} 24$ & $\mathrm{C} 21$ & $\mathrm{C} 22$ & $104.8(3)$ & $\mathrm{C} 28$ & $\mathrm{C} 27$ & $\mathrm{C} 26$ & $121.0(3)$ \\
\hline $\mathrm{C} 6$ & $\mathrm{C} 1$ & Au1 & $113.9(2)$ & F6 & $\mathrm{C} 8 \mathrm{~A}$ & C9A & $115.2(3)$ \\
\hline $\mathrm{C} 2$ & $\mathrm{C} 1$ & $\mathrm{Au} 1$ & $127.3(3)$ & F6 & $\mathrm{C} 8 \mathrm{~A}$ & C7A & $119.0(3)$ \\
\hline $\mathrm{C} 2$ & $\mathrm{C} 1$ & $\mathrm{C} 6$ & $118.7(3)$ & C9A & $\mathrm{C} 8 \mathrm{~A}$ & C7A & $125.8(3)$ \\
\hline $\mathrm{C} 38$ & $\mathrm{C} 37$ & $\mathrm{~N} 2$ & 121.2(3) & $\mathrm{C} 9$ & $\mathrm{C} 10$ & $\mathrm{C} 11$ & $120.3(3)$ \\
\hline $\mathrm{C} 38$ & $\mathrm{C} 37$ & $\mathrm{C} 42$ & 124.1(3) & F15 & $\mathrm{C} 18 \mathrm{~A}$ & $\mathrm{C} 13 \mathrm{~A}$ & 121.1(3) \\
\hline $\mathrm{C} 42$ & $\mathrm{C} 37$ & $\mathrm{~N} 2$ & $114.6(3)$ & F15 & $\mathrm{C} 18 \mathrm{~A}$ & $\mathrm{C} 17 \mathrm{~A}$ & $115.3(3)$ \\
\hline C16 & $\mathrm{C} 15$ & $\mathrm{C} 14$ & $105.8(3)$ & $\mathrm{C} 17 \mathrm{~A}$ & $\mathrm{C} 18 \mathrm{~A}$ & $\mathrm{C} 13 \mathrm{~A}$ & $123.5(4)$ \\
\hline
\end{tabular}




\begin{tabular}{|c|c|c|c|c|c|c|c|}
\hline F20 & $\mathrm{C} 24 \mathrm{~A}$ & $\mathrm{C} 19 \mathrm{~A}$ & $121.2(3)$ & F2 & $\mathrm{C} 3 \mathrm{~A}$ & $\mathrm{C} 4 \mathrm{~A}$ & $119.7(3)$ \\
\hline F20 & $\mathrm{C} 24 \mathrm{~A}$ & $\mathrm{C} 23 \mathrm{~A}$ & $114.3(3)$ & F2 & $\mathrm{C} 3 \mathrm{~A}$ & $\mathrm{C} 2 \mathrm{~A}$ & $120.5(3)$ \\
\hline C19A & $\mathrm{C} 24 \mathrm{~A}$ & $\mathrm{C} 23 \mathrm{~A}$ & $124.4(3)$ & $\mathrm{C} 2 \mathrm{~A}$ & $\mathrm{C} 3 \mathrm{~A}$ & $\mathrm{C} 4 \mathrm{~A}$ & $119.7(3)$ \\
\hline $\mathrm{C} 12$ & $\mathrm{C} 11$ & $\mathrm{C} 10$ & $120.1(3)$ & $\mathrm{F} 1$ & $\mathrm{C} 2 \mathrm{~A}$ & $\mathrm{C} 1 \mathrm{~A}$ & $119.4(3)$ \\
\hline F7 & C9A & $\mathrm{C} 10 \mathrm{~A}$ & 119.3(3) & $\mathrm{F} 1$ & $\mathrm{C} 2 \mathrm{~A}$ & $\mathrm{C} 3 \mathrm{~A}$ & $116.5(3)$ \\
\hline F7 & C9A & $\mathrm{C} 8 \mathrm{~A}$ & $121.8(3)$ & $\mathrm{C} 3 \mathrm{~A}$ & $\mathrm{C} 2 \mathrm{~A}$ & $\mathrm{C} 1 \mathrm{~A}$ & $124.1(3)$ \\
\hline $\mathrm{C} 8 \mathrm{~A}$ & C9A & $\mathrm{C} 10 \mathrm{~A}$ & $118.9(3)$ & $\mathrm{C} 42$ & $\mathrm{C} 43$ & $\mathrm{C} 44$ & $112.8(3)$ \\
\hline F10 & $\mathrm{C} 12 \mathrm{~A}$ & $\mathrm{C} 11 \mathrm{~A}$ & $114.8(3)$ & $\mathrm{C} 42$ & $\mathrm{C} 43$ & $\mathrm{C} 45$ & $112.6(3)$ \\
\hline F10 & $\mathrm{C} 12 \mathrm{~A}$ & C7A & $121.6(3)$ & $\mathrm{C} 44$ & $\mathrm{C} 43$ & $\mathrm{C} 45$ & $108.6(3)$ \\
\hline $\mathrm{C} 11 \mathrm{~A}$ & $\mathrm{C} 12 \mathrm{~A}$ & $\mathrm{C} 7 \mathrm{~A}$ & $123.5(3)$ & $\mathrm{C} 4$ & $\mathrm{C} 5$ & C6 & $119.7(3)$ \\
\hline F8 & $\mathrm{C} 10 \mathrm{~A}$ & C11A & 121.3(3) & $\mathrm{C} 40$ & $\mathrm{C} 41$ & $\mathrm{C} 42$ & $120.9(4)$ \\
\hline F8 & $\mathrm{C} 10 \mathrm{~A}$ & C9A & $120.1(3)$ & F12 & $\mathrm{C} 15 \mathrm{~A}$ & C14A & 121.1(3) \\
\hline $\mathrm{C} 11 \mathrm{~A}$ & $\mathrm{C} 10 \mathrm{~A}$ & C9A & $118.5(3)$ & F12 & $\mathrm{C} 15 \mathrm{~A}$ & C16A & $120.0(3)$ \\
\hline $\mathrm{C} 1$ & $\mathrm{C} 6$ & $\mathrm{C} 7$ & $113.8(3)$ & $\mathrm{C} 16 \mathrm{~A}$ & $\mathrm{C} 15 \mathrm{~A}$ & $\mathrm{C} 14 \mathrm{~A}$ & $118.8(4)$ \\
\hline $\mathrm{C} 5$ & C6 & $\mathrm{C} 1$ & $120.3(3)$ & $\mathrm{C} 10$ & $\mathrm{C} 9$ & $\mathrm{C} 8$ & $119.4(3)$ \\
\hline $\mathrm{C} 5$ & $\mathrm{C} 6$ & $\mathrm{C} 7$ & $125.3(3)$ & $\mathrm{C} 9$ & $\mathrm{C} 8$ & $\mathrm{C} 7$ & $120.8(3)$ \\
\hline C37 & $\mathrm{C} 38$ & $\mathrm{C} 46$ & $125.0(3)$ & F13 & $\mathrm{C} 16 \mathrm{~A}$ & $\mathrm{C} 15 \mathrm{~A}$ & $120.2(4)$ \\
\hline C39 & C38 & C37 & $116.4(3)$ & F13 & $\mathrm{C} 16 \mathrm{~A}$ & C17A & $120.4(3)$ \\
\hline C39 & $\mathrm{C} 38$ & $\mathrm{C} 46$ & $118.4(3)$ & $\mathrm{C} 17 \mathrm{~A}$ & $\mathrm{C} 16 \mathrm{~A}$ & $\mathrm{C} 15 \mathrm{~A}$ & $119.4(3)$ \\
\hline $\mathrm{C} 24 \mathrm{~A}$ & C19A & $\mathrm{C} 20 \mathrm{~A}$ & $112.8(3)$ & $\mathrm{C} 27$ & $\mathrm{C} 28$ & $\mathrm{C} 29$ & $121.2(3)$ \\
\hline $\mathrm{C} 24 \mathrm{~A}$ & $\mathrm{C} 19 \mathrm{~A}$ & $\mathrm{~B} 1$ & $128.1(3)$ & F14 & C17A & C18A & $119.4(4)$ \\
\hline $\mathrm{C} 20 \mathrm{~A}$ & C19A & $\mathrm{B} 1$ & 119.0(3) & F14 & C17A & C16A & $120.4(3)$ \\
\hline C14A & $\mathrm{C} 13 \mathrm{~A}$ & $\mathrm{C} 18 \mathrm{~A}$ & $112.8(3)$ & $\mathrm{C} 16 \mathrm{~A}$ & C17A & $\mathrm{C} 18 \mathrm{~A}$ & $120.2(3)$ \\
\hline C14A & $\mathrm{C} 13 \mathrm{~A}$ & $\mathrm{~B} 1$ & $119.4(3)$ & $\mathrm{C} 4$ & $\mathrm{C} 3$ & $\mathrm{C} 2$ & $120.5(4)$ \\
\hline C18A & $\mathrm{C} 13 \mathrm{~A}$ & B1 & $127.0(3)$ & $\mathrm{C} 3$ & $\mathrm{C} 4$ & $\mathrm{C} 5$ & $120.2(3)$ \\
\hline F4 & $\mathrm{C} 5 \mathrm{~A}$ & C6A & $120.7(3)$ & $\mathrm{C} 40$ & C39 & $\mathrm{C} 38$ & $121.3(4)$ \\
\hline F4 & $\mathrm{C} 5 \mathrm{~A}$ & $\mathrm{C} 4 \mathrm{~A}$ & $120.2(3)$ & C39 & $\mathrm{C} 40$ & $\mathrm{C} 41$ & $121.2(4)$ \\
\hline $\mathrm{C} 4 \mathrm{~A}$ & $\mathrm{C} 5 \mathrm{~A}$ & C6A & 119.1(3) & $\mathrm{C} 19 \mathrm{~A}$ & B1 & $\mathrm{C} 13 \mathrm{~A}$ & $112.7(3)$ \\
\hline F19 & $\mathrm{C} 23 \mathrm{~A}$ & $\mathrm{C} 24 \mathrm{~A}$ & $120.7(3)$ & $\mathrm{C} 19 \mathrm{~A}$ & B1 & $\mathrm{C} 7 \mathrm{~A}$ & $114.2(3)$ \\
\hline F19 & $\mathrm{C} 23 \mathrm{~A}$ & $\mathrm{C} 22 \mathrm{~A}$ & $119.7(3)$ & $\mathrm{C} 19 \mathrm{~A}$ & B1 & $\mathrm{C} 1 \mathrm{~A}$ & $102.8(3)$ \\
\hline $\mathrm{C} 22 \mathrm{~A}$ & $\mathrm{C} 23 \mathrm{~A}$ & $\mathrm{C} 24 \mathrm{~A}$ & $119.6(3)$ & C7A & B1 & $\mathrm{C} 13 \mathrm{~A}$ & $100.1(3)$ \\
\hline F18 & $\mathrm{C} 22 \mathrm{~A}$ & $\mathrm{C} 23 \mathrm{~A}$ & $120.6(4)$ & $\mathrm{C} 1 \mathrm{~A}$ & B1 & C13A & $114.5(3)$ \\
\hline F18 & $\mathrm{C} 22 \mathrm{~A}$ & $\mathrm{C} 21 \mathrm{~A}$ & $120.4(3)$ & $\mathrm{C} 1 \mathrm{~A}$ & B1 & C7A & $113.0(3)$ \\
\hline $\mathrm{C} 21 \mathrm{~A}$ & $\mathrm{C} 22 \mathrm{~A}$ & $\mathrm{C} 23 \mathrm{~A}$ & $119.0(3)$ & $\mathrm{C} 1 \mathrm{~B}$ & $\mathrm{C} 2 \mathrm{~B}$ & C3B & $118.4(6)$ \\
\hline $\mathrm{C} 12$ & $\mathrm{C} 7$ & C6 & $114.9(3)$ & $\mathrm{C} 4 \mathrm{~B}$ & $\mathrm{C} 3 \mathrm{~B}$ & $\mathrm{C} 2 \mathrm{~B}$ & $121.0(6)$ \\
\hline $\mathrm{C} 8$ & $\mathrm{C} 7$ & $\mathrm{C} 12$ & 119.1(3) & C5B & C4B & $\mathrm{C} 3 \mathrm{~B}$ & $120.1(6)$ \\
\hline $\mathrm{C} 8$ & $\mathrm{C} 7$ & C6 & $125.4(3)$ & $\mathrm{C} 4 \mathrm{~B}$ & $\mathrm{C} 5 \mathrm{~B}$ & $\mathrm{C} 6 \mathrm{~B}$ & $119.2(6)$ \\
\hline C38 & $\mathrm{C} 46$ & $\mathrm{C} 48$ & 109.6(3) & $\mathrm{C} 5 \mathrm{~B}$ & $\mathrm{C} 6 \mathrm{~B}$ & $\mathrm{C} 1 \mathrm{~B}$ & $121.5(6)$ \\
\hline C38 & $\mathrm{C} 46$ & $\mathrm{C} 47$ & $112.8(3)$ & $\mathrm{C} 2 \mathrm{~B}$ & $\mathrm{C} 1 \mathrm{~B}$ & $\mathrm{C} 6 \mathrm{~B}$ & $119.7(6)$ \\
\hline C47 & $\mathrm{C} 46$ & C48 & $108.6(3)$ & & & & \\
\hline
\end{tabular}


Table 6.4.4. Torsion angles for 7

\begin{tabular}{|c|c|c|c|c|c|c|c|c|c|}
\hline A & B & C & D & Angle $/^{\circ}$ & A & B & & D & ngle $/^{\circ}$ \\
\hline Aul & N1 & $\mathrm{C} 20$ & $\mathrm{C} 14$ & $1.7(4)$ & $\mathrm{C} 12 \mathrm{~A}$ & $\mathrm{C} 7 \mathrm{~A}$ & C8A & C9A & $0.3(5)$ \\
\hline u1 & N1 & $\mathrm{C} 20$ & $\mathrm{C} 21$ & $-177.1(2)$ & $\mathrm{C} 12 \mathrm{~A}$ & $\mathrm{C} 7 \mathrm{~A}$ & $\mathrm{~B} 1$ & C19A & A-137.3(3) \\
\hline $\mathrm{u} 1$ & N1 & $\mathrm{C} 25$ & $\mathrm{C} 30$ & -95. & $\mathrm{C} 12 \mathrm{~A}$ & $\mathrm{C} 7 \mathrm{~A}$ & B1 & C13A & A 102. \\
\hline u1 & N1 & $\mathrm{C} 25$ & $\mathrm{C} 26$ & 80. & & $\mathrm{C} 7 \mathrm{~A}$ & B1 & $\mathrm{C} 1 \mathrm{~A}$ & \\
\hline $\mathrm{u} 1$ & $\mathrm{C} 13$ & $\mathrm{C} 14$ & $\mathrm{C} 20$ & -37.6 & $\mathrm{C} 10 \mathrm{AC}$ & $\mathrm{IC} 11 \mathrm{AC}$ & $\mathrm{C} 12 \mathrm{~A}$ & A F10 & -178.3 \\
\hline Au1 & $\mathrm{C} 13$ & $\mathrm{C} 14$ & $\mathrm{C} 15$ & $-16^{\prime}$ & $\mathrm{C} 10 \mathrm{AC}$ & $\mathrm{CC} 11 \mathrm{AC}$ & $\mathrm{C} 12 \mathrm{~A}$ & A C7A & $0.3(5)$ \\
\hline $\mathrm{u} 1$ & $\mathrm{C} 13$ & $\mathrm{C} 14$ & $\mathrm{C} 19$ & 76.2 & $\mathrm{C} 10 \mathrm{~A}$ & C9A & $\mathrm{C} 8 \mathrm{~A}$ & F6 & $175.6(3)$ \\
\hline $\mathrm{u} 1$ & $\mathrm{C} 12$ & $\mathrm{C} 11$ & $\mathrm{C} 10$ & 173 & $\mathrm{C} 10 \mathrm{~A}$ & C9A & C8A & C7A & -1.4 \\
\hline u1 & $\mathrm{C} 12$ & $\mathrm{C} 7$ & C6 & 15.6 & C6 & $\mathrm{C} 1$ & $\mathrm{C} 2$ & $\mathrm{C} 3$ & $5.0(5)$ \\
\hline Aul & $\mathrm{C} 12$ & $\mathrm{C} 7$ & $\mathrm{C} 8$ & $-172.4(3)$ & C6 & $\mathrm{C} 7$ & $\mathrm{C} 8$ & C9 & $168.0(4)$ \\
\hline u1 & $\mathrm{C} 1$ & $\mathrm{C} 6$ & $\mathrm{C} 7$ & -13 & $\mathrm{C} 6$ & $\mathrm{C} 5$ & $\mathrm{C} 4$ & $\mathrm{C} 3$ & $2 .{ }^{\circ}$ \\
\hline $\mathrm{u} 1$ & $\mathrm{C} 1$ & C6 & $\mathrm{C} 5$ & & 38 & C37 & $\mathrm{C} 42$ & $\mathrm{C} 43$ & \\
\hline u1 & $\mathrm{C} 1$ & $\mathrm{C} 2$ & $\mathrm{C} 3$ & $-177.3(3)$ & C38 & C37 & $\mathrm{C} 42$ & $\mathrm{C} 41$ & $2.8(5)$ \\
\hline F20 & $\mathrm{C} 24 \mathrm{~A}$ & C19A & $\mathrm{C} 20 \mathrm{~A}$ & $1-17$ & $\mathrm{C} 38$ & C39 & $\mathrm{C} 40$ & $\mathrm{C} 41$ & $1.5(6)$ \\
\hline 20 & C24 & C19A & $\mathrm{B} 1$ & $-0.4(5)$ & C19A & 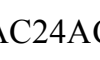 & $\mathrm{C} 23 \mathrm{~A}$ & F19 & $-179.7(3)$ \\
\hline 20 & $\mathrm{C} 24$ & $23 \mathrm{~A}$ & F19 & $-0.1(4)$ & $\mathrm{C} 1$ & $\mathrm{C} 2$ & 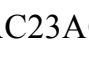 & $\mathrm{AC} 22$ & $.0(5)$ \\
\hline F20 & C24A & $\mathrm{C} 23 \mathrm{~A}$ & $\mathrm{C} 22 \mathrm{~A}$ & $179.6(3)$ & C19A & $\mathrm{IC} 20 \mathrm{AC}$ & $\mathrm{C} 21 \mathrm{~A}$ & F17 & $-178.6(3)$ \\
\hline F7 & C9A & $\mathrm{C} 10 \mathrm{~A}$ & $\mathrm{~F} 8$ & $0.4(4)$ & $\mathrm{C} 19 \mathrm{~A}$ & $\mathrm{C} 20 \mathrm{AC}$ & A & $\mathrm{AC} 22 \mathrm{~A}$ & 2.2(6) \\
\hline F7 & $\mathrm{C} 9$ & $\mathrm{C} 10 \mathrm{~A}$ & $\mathrm{C} 11$ & & & & & & \\
\hline F7 & C9A & C8A & F6 & $-3.5(4)$ & $\mathrm{C} 13 \mathrm{AC}$ & ( & 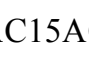 & $\mathrm{AC} 16 \mathrm{~A}$ & A $1.4(6)$ \\
\hline F7 & C9A & C8A & C7A & $179.4(3)$ & $\mathrm{C} 13 \mathrm{~A}$ & $\mathrm{C} 18 \mathrm{AC}$ & $\mathrm{C}$ & F14 & $-178.7(3)$ \\
\hline F10 & $\mathrm{C} 12 \mathrm{~A}$ & C7A & $\mathrm{C} 8 \mathrm{~A}$ & 178 & $\mathrm{C} 13 \mathrm{AC}$ & $\mathrm{C} 1$ & $\mathrm{C}$ & $\mathrm{AC} 16 \mathrm{~A}$ & A $3.5(6)$ \\
\hline F10 & $\mathrm{C} 12$ & C7A & B1 & & & C & . & $\mathrm{C} 2 \mathrm{~A}$ & 1. \\
\hline F9 & $\mathrm{C} 11 \mathrm{~A}$ & C12A & $\mathrm{F} 10$ & $0.1(4)$ & $\mathrm{C} 5 \mathrm{~A}$ & $\mathrm{C} 6 \mathrm{~A}$ & $\mathrm{C} 1 \mathrm{~A}$ & B1 & $175.7(3)$ \\
\hline F9 & C11A & C12A & $\mathrm{C} 7 \mathrm{~A}$ & $178.6(3)$ & C5A & $\mathrm{C} 4 \mathrm{~A}$ & $\mathrm{C} 3 \mathrm{~A}$ & $\mathrm{~F} 2$ & $179.6(3)$ \\
\hline F9 & $\mathrm{C} 11$ & CIOA & F8 & $0.9(5)$ & e & 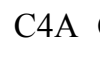 & $\mathrm{C}$ & $\mathrm{C} 2 \mathrm{~A}$ & (5) \\
\hline F9 & C11A & $\mathrm{C} 10 \mathrm{~A}$ & $\mathrm{C} 9 \mathrm{~A}$ & -179 & $\mathrm{C} 23 \mathrm{AC}$ & $\mathrm{C} 24 \mathrm{AC}$ & C19A & $\mathrm{AC} 20 \mathrm{~A}$ & A $2.9(5)$ \\
\hline F11 & C14A & C15A & F12 & $0.7(5)$ & $\mathrm{C} 23 \mathrm{AC}$ & $\mathrm{C} 24 \mathrm{AC}$ & C19A & B1 & 179.2(3) \\
\hline F11 & C14A & $\mathrm{C} 15 \mathrm{~A}$ & $\mathrm{C} 16 \mathrm{~A}$ & 7(3) & $\mathrm{C} 23 \mathrm{AC}$ & $\mathrm{C} 22 \mathrm{AC}$ & C21A & F17 & $-178.0(3)$ \\
\hline 16 & $\mathrm{C} 2$ & 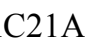 & F17 & -0. & $\mathrm{C} 23 \mathrm{AC}$ & $\mathrm{C} 2$ & $\mathrm{C} 21 \mathrm{~A}$ & $\mathrm{AC} 20 \mathrm{~A}$ & A $1.1(5)$ \\
\hline F16 & $\mathrm{C} 20 \mathrm{~A}$ & $\mathrm{C} 21 \mathrm{~A}$ & $\mathrm{C} 22 \mathrm{~A}$ & $-179.2(3)$ & $\mathrm{C} 7$ & $\mathrm{C} 12$ & $\mathrm{C} 11$ & $\mathrm{C} 10$ & $-3.3(6)$ \\
\hline F15 & C18A & C17A & F14 & $1.7(5)$ & $\mathrm{C} 7$ & C6 & $\mathrm{C} 5$ & $\mathrm{C} 4$ & $-166.7(4)$ \\
\hline F15 & $\mathrm{C} 18$ & $\mathrm{C} 17 \mathrm{~A}$ & $\mathrm{C} 16$ & $-1 /$ & C46 & C38 & C39 & $\mathrm{C} 40$ & $-173.2(3)$ \\
\hline F5 & C6A & $\mathrm{C} 1 \mathrm{~A}$ & $\mathrm{C} 2 \mathrm{~A}$ & -17 & C30 & $\mathrm{C} 25$ & $\mathrm{C} 26$ & $\mathrm{C} 34$ & $173.7(3)$ \\
\hline F5 & C6A & $\mathrm{C} 1 \mathrm{~A}$ & B1 & $-3.5(5)$ & 30 & $\mathrm{C} 25$ & $\mathrm{C} 26$ & $\mathrm{C} 27$ & $-4.7(5)$ \\
\hline F19 & C23A & C22A & $\mathrm{F} 18$ & $-0.9(5)$ & $\mathrm{C} 30$ & $\mathrm{C} 29$ & $\mathrm{C} 28$ & $\mathrm{C} 27$ & $-3.0(6)$ \\
\hline F19 & $\mathrm{C} 23 \mathrm{~A}$ & $\mathrm{C} 22 \mathrm{~A}$ & $\mathrm{C} 21 \mathrm{~A}$ & $177.5(3)$ & $\mathrm{C} 25$ & $\mathrm{~N} 1$ & $\mathrm{C} 20$ & $\mathrm{C} 14$ & $175.4(3)$ \\
\hline F18 & $\mathrm{C} 22 \mathrm{~A}$ & $\mathrm{C} 21 \mathrm{~A}$ & $\mathrm{~F} 17$ & $0.4(5)$ & $\mathrm{C} 25$ & N1 & $\mathrm{C} 20$ & $\mathrm{C} 21$ & $-3.4(5)$ \\
\hline F18 & 2 & 21 & $\mathrm{C} 20$ & 7 & $\mathrm{C} 25$ & $\mathrm{C} 30$ & $\mathrm{C} 31$ & $\mathrm{C} 3$ & $-135.7(4)$ \\
\hline 12 & $15 \mathrm{~A}$ & C16A & F13 & $-0.3(5)$ & $\mathrm{C} 25$ & $\mathrm{C} 30$ & $\mathrm{C} 31$ & C32 & $100.0(4)$ \\
\hline
\end{tabular}




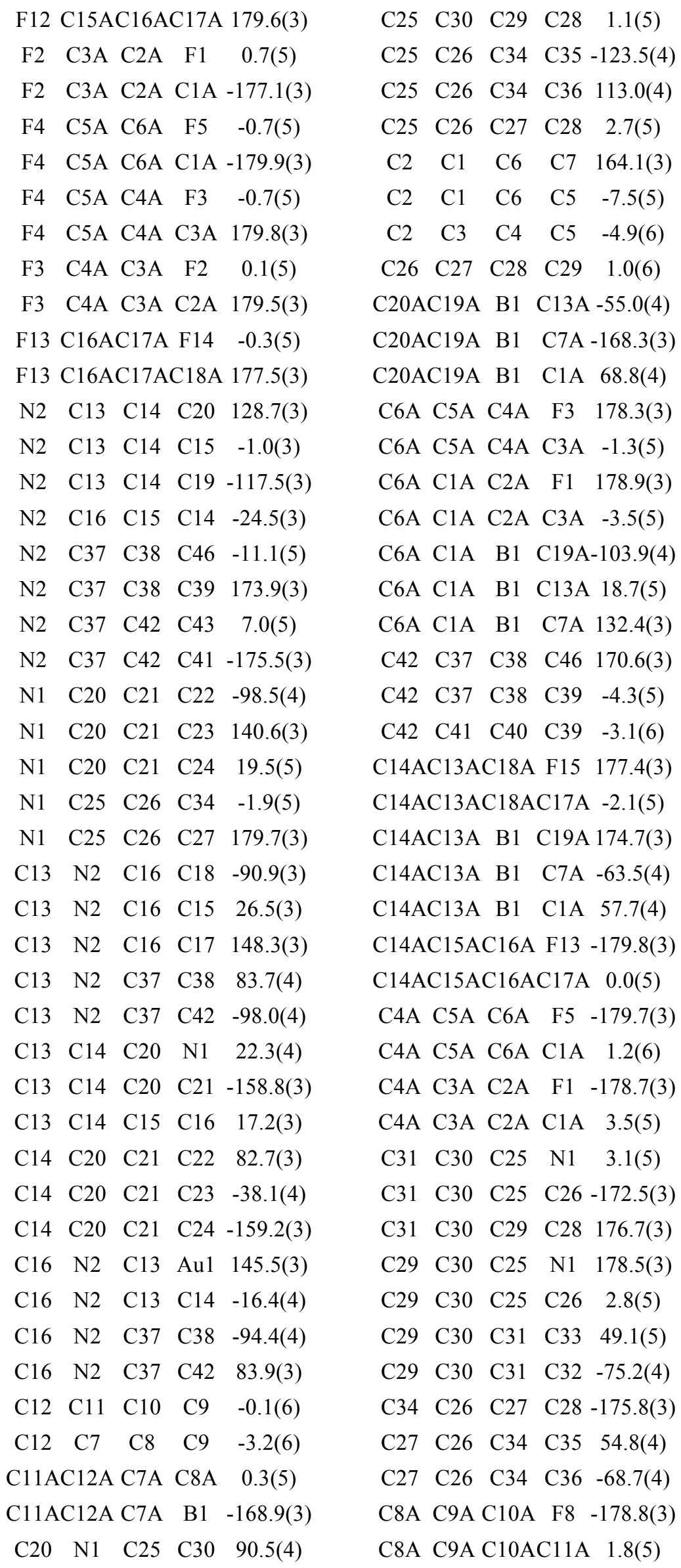




\begin{tabular}{|c|c|c|c|c|}
\hline 20 & N1 & $25 \mathrm{C} 26$ & 91 & C8A C7A B1 C19A 5 \\
\hline $\mathrm{C} 20$ & $\mathrm{C} 14$ & $\mathrm{C} 15 \mathrm{C} 16$ & $-105.7(3)$ & C8A C7A B1 C13A -66.4(4) \\
\hline $\mathrm{C} 1$ & C6 & $\mathrm{C} 7 \mathrm{C} 12$ & $-0.9(5)$ & C8A C7A B1 C1A 171.4(3) \\
\hline $\mathrm{C} 1$ & C6 & $\mathrm{C} 7$ & -172 & $\mathrm{C} 10 \quad \mathrm{C} 9$ \\
\hline $\mathrm{C} 1$ & C6 & $\mathrm{C} 4$ & 3 & $18 \mathrm{AC} 13 \mathrm{AC} 14 \mathrm{~A} F 11-1$ \\
\hline $\mathrm{C} 1$ & $\mathrm{C} 2$ & $\mathrm{C} 4$ & 1 . & $\mathrm{C} 18 \mathrm{AC} 13 \mathrm{AC} 14 \mathrm{AC} 15 \mathrm{~A}-0.3(5)$ \\
\hline 18 & $\mathrm{C} 16$ & $\mathrm{C} 15 \mathrm{C} 14$ & 88.5 & C18AC13A B1 C19A-16.4(5) \\
\hline 37 & $\mathrm{~N} 2$ & C13 Au1 & $-32.7(5)$ & C18AC13A B1 C7A 105.4(4) \\
\hline 37 & $\mathrm{~N} 2$ & $\mathrm{C} 13 \mathrm{C} 14$ & 165 & C18AC13A B1 C1A -133.4(4 \\
\hline 37 & N2 & $\mathrm{C} 16 \mathrm{C} 18$ & 871 & C2A C1A B1 C19A 70.4(4) \\
\hline $\mathrm{C} 37$ & $\mathrm{~N} 2$ & $\mathrm{C} 16 \mathrm{C} 15$ & -15 & C2A C1A B1 C13A-167.0(3) \\
\hline $\mathrm{C} 37$ & $\mathrm{~N} 2$ & $\mathrm{C} 16 \mathrm{C} 17$ & -33 & C2A C1A B1 C7A -53.3(4) \\
\hline 37 & $\mathrm{C} 38$ & $\mathrm{C} 46 \mathrm{C} 48$ & -112 & C43 C42 C41 C40 178.6(3) \\
\hline $\mathrm{C} 37$ & $\mathrm{C} 38$ & $\mathrm{C} 46 \mathrm{C} 47$ & 126. & C7 C12 170.2(4) \\
\hline C37 & $\mathrm{C} 38$ & C39 C40 & 2.1 & $\mathrm{C} 8 \quad-1.3(6)$ \\
\hline $\mathrm{C} 37$ & $\mathrm{C} 42$ & $\mathrm{C} 43 \mathrm{C} 44$ & -137 & $\mathrm{C} 41 \quad \mathrm{C} 42 \quad \mathrm{C} 43 \quad \mathrm{C} 44 \quad 45.1(4)$ \\
\hline $\mathrm{C} 37$ & $\mathrm{C} 42$ & $\mathrm{C} 43 \mathrm{C} 45$ & & C41 C42 C43 C45 $\quad-7$ \\
\hline 37 & $\mathrm{C} 42$ & C41 C40 & 1.0 & $15 \mathrm{AC} 16 \mathrm{AC} 17 \mathrm{~A} \mathrm{~F} 14$ 179.9(3) \\
\hline 15 & $\mathrm{C} 14$ & $\mathrm{C} 20 \quad \mathrm{~N} 1$ & 1 & $\mathrm{C} 15 \mathrm{AC} 16 \mathrm{AC} 17 \mathrm{AC} 18 \mathrm{~A} \quad-2.3(6)$ \\
\hline 15 & C14 & $\mathrm{C} 20 \mathrm{C} 21$ & -38 & $\begin{array}{lllll}\text { C39 } & \text { C38 } & \text { C46 } & \text { C48 } & 62.3(4)\end{array}$ \\
\hline 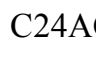 & $\mathrm{C}$ & F16 & 177 & $\begin{array}{llllll}\mathrm{C} 39 & \mathrm{C} 38 & \mathrm{C} 46 & \mathrm{C} 47 & -58.8(4)\end{array}$ \\
\hline & $\mathrm{C} 19$ & 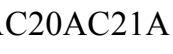 & & B1 C19AC20A F16 \\
\hline $2-1$ & $19 \mathrm{~A}$ & $\mathrm{~B} 1 \mathrm{C}$ & & B1 C19AC20AC2 \\
\hline 24 & $C 10$ & B1 C7A & 15 & B1 C13AC14A F11 -8 \\
\hline & 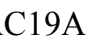 & $\mathrm{B} 1 \mathrm{C} 1 \mathrm{~A}$ & -10 & B1 C13AC14AC15A 170.1(3) \\
\hline 2 & CLSAC & 8 & 179 & B1 C13AC18A F15 7.9(5) \\
\hline $\mathrm{C} 24$ & $\mathrm{C}$ & $\mathrm{C} 21 \mathrm{~A}$ & $-2.2(5)$ & B1 C13AC18AC17A-171.6(3 \\
\hline $\mathrm{C} 19$ & $\mathrm{C} 14$ & $\mathrm{C} 20 \mathrm{~N} 1$ & $-89.1(3)$ & B1 C7A C8A F6 $\quad-6.7(5)$ \\
\hline 10 & $\mathrm{C} 14$ & $\mathrm{C} 20 \mathrm{C} 21$ & . & 31 C7A C8A C9A 170.2(3) \\
\hline $\mathrm{C} 19$ & $\mathrm{C} 14$ & $\mathrm{C} 15 \mathrm{C} 16$ & 127 & B1 C1A C2A F1 \\
\hline $\mathrm{C} 11$ & $\mathrm{C} 12$ & $\mathrm{C} 7$ & -167. & 31 C1A C2A C3A -178 \\
\hline 11 & $\mathrm{C} 12$ & $\mathrm{C} 8$ & ) & 2B C3B C4B C5B $0.3(9)$ \\
\hline $\mathrm{C} 11$ & $\mathrm{C} 10$ & $\mathrm{C} 8$ & 6) & С3B C2B C1B C6B $-0.1(9)$ \\
\hline $\mathrm{C} 17$ & $\mathrm{C} 16$ & $\mathrm{C} 15 \mathrm{C} 14$ & $-147.0(3)$ & C3B C4B C5B C6B $-0.5(9)$ \\
\hline \multicolumn{3}{|c|}{ C12AC11AC10A F8 } & 179.3(3) & C4B C5B C6B C1B $0.5(9)$ \\
\hline \multicolumn{3}{|c|}{ C12AC11AC10A C9A } & $-1.3(5)$ & C5B C6B C1B C2B $-0.2(9)$ \\
\hline \multicolumn{3}{|c|}{ C12A C7A C8A } & $-176.6(3)$ & C1B C2B C3B C4B $0.0(9)$ \\
\hline
\end{tabular}




\section{References}

1) Tolentino, D. R.; Jin, L.; Melaimi, M.; Bertrand, G. Chem. Asian J. 2015, 10, 2139.

2) Hu, X.; Soleihavoup, M.; Melaimi, M.; Chu, J.; Bertrand, G. Angew. Chem., Int. Ed. 2015, 54,6008 .

3) Jin, L.; Tolentino, D. R.; Melaimi, M.; Bertrand, G. Sci. Adv. 2015, 1, e1500304.

4) Jin, L.; Romero, E. A.; Melaimi, M.; Bertrand, G. J. Am. Chem. Soc. 2015, 137, 15696.

5) Karakus, C.; Fischer, L. H.; Schmeding, S.; Hummel, J.; Risch, N.; Schäferling, M.;

Holder, E. Dalton Trans. 2012, 41, 9623.

6) Budzelaar, P. H. M.; Oort, A. B.; Orper, A. G. Eur. J. Inorg. Chem. 1998, 1485.

7) Kabir, S. M. H.; Hasegawa, M.; Kuwatani, Y.; Yoshida, M.; Matsuyama, H.; Iyoda, M. J.

Chem. Soc. Perkin Trans. 2001, 1, 159.

8) Dolomanov O.V., Bourhis L.J., Gildea R.J., Howard J.A.K., Puschmann H. J. Appl. Cryst. 2009, 42, 339.

9) G. M. Sheldrick, Acta Cryst. 2008, A64, 112. 